\title{
ELECTRONIC AND MAGNETIC PROPERTIES OF TWO DIMENSIONAL CRYSTALS
}

BY

HAN I HATAMI

\begin{abstract}
A thesis
submitted to the Victoria University of Wellington in fulfilment of the requirements for the degree of Doctor of Philosophy
\end{abstract}

Victoria University of Wellington 2015 
Hani Hatami: Electronic and Magnetic Properties of Two Dimensional Crystals, (C) 2015 
I dedicate this thesis to my beautiful wife Leila. 



\section{ABSTRACT}

In the last few years, two dimensional crystals have become available for experimental studies. Good examples of such systems are monolayers and bilayers of graphene and monolayers of transition metal dichalcogenides such as $\mathrm{MoS}_{2}$ and $\mathrm{WSe}_{2}$. The availability of two dimensional crystals has encouraged physicists to study the electronic and magnetic properties of such systems. This thesis adds to the theoretical knowledge about electronic and magnetic properties of two dimensional crystals with the focus on graphene and $\mathrm{MoS}_{2}$.

As a general theme in this thesis, we calculate how in general these systems interact with electric and magnetic fields and what their response is to such stimuli. In particular, we have studied the response of monolayer graphene to an in-plane electric field. We have also looked at spin-orbit coupling effects that arise from applying perpendicular or in-plane external electric fields, especially their consequences for transport properties of bilayer graphene. We investigated the electronic properties of charge carriers confined in a mesoscopic ring structure using a gate voltage in bilayer graphene. We also showed how spin-orbit coupling can affect the electrical properties of such rings. We found how spin-orbit coupling can affect the transport properties in bilayer graphene. We also investigated the RKKY or indirect exchange coupling between magnetic moments in monolayer $\mathrm{MoS}_{2}$ through calculating wave vector dependent spin susceptibility.

We examined the electronic properties of electrons and holes confined electrostatically into a bilayer graphene ring. We presented an analytical solution for finding energy levels in the ring. We showed that the magnetic field dependence of the lowest energy level with fixed angular momentum in bilayer graphene rings, in contrast to usual semiconductor quantum rings, is not parabolic but displays an asymmetric "Mexican hat". We found that introducing spin-orbit coupling in the ring can flatten this Mexican hat. 
We studied the effect of an orbital Rashba type effect, induced by an in-plane electric field in monolayer graphene. Using perturbation theory, we showed that this term can affect the energy levels in a crossed electric and magnetic field such that the electron and hole levels repel each other. We calculated the AC transport of monolayer graphene in the linear-response regime and showed that taking the orbital Rashba term into account casts doubt on the universality of the minimum conductivity of monolayer graphene.

We studied the effect of spin-orbit coupling in transport properties of bilayer graphene systems by calculating tunnelling through npn and np junctions. We showed that at sufficiently large spin-orbit strength, normal transmission through a barrier which is forbidden in bilayer graphene becomes finite. We predict that in a weak Rashba spin-orbit regime, outgoing electrons show signals which are spin polarized. We also showed that considering spin-orbit coupling only in the barrier of an npn junction can invert the spin of the incoming electrons.

Finally, we obtained analytical expressions for the wave vector-dependent static spin susceptibility of monolayer transition metal dichalcogenides, considering both the electron-doped and hole-doped cases. These results are then applied to the calculations of physical observables of monolayer $\mathrm{MoS}_{2}$. We claculated that the hole-mediated RKKY exchange interaction for in-plane impurity-spin components decays with a different power law from what is expected for a twodimensional Fermi liquid. In contrast, we calculated that the out-of-plane spin response shows the conventional long-range behaviour. 
I would never have been able to finish my thesis without the guidance of my supervisor, help from my friends, and support from my family and wife.

\section{ACKNOWLEDGMENTS}

I express my deepest gratitude to my primary supervisor, Prof. Uli Zuelicke, for his excellent guidance, immense knowledge, caring and patience. My sincere thanks also goes to Dr. Michele Governale for accepting to be my secondary supervisor and also being always supportive and helpful. I also thank Dr. Thomas Kernreiter who I had the chance to enjoy working with him on the last project of my PhD studies.

I thank Dr. Alireza Qaiumzadeh, who as a good friend, was always willing to help and give his best suggestions. Many thanks to Peter Hauer, Christopher Galloway, Chun Y. Cheah, Stefan Hertel, Stephanie Droste, Benny Darby and other friends for being by my side and always giving me reasons to cheer.

I also thank my parents and two younger brothers. They were always supporting me and encouraging me with their best wishes.

Last but not least, I thank my lovely wife Leila whose patient love enabled me to complete this work. Her support and encouragement was in the end what made this dissertation possible. 



\section{CONTENTS}

I REVIEW OF ELECTRONIC PROPERTIES OF TWO DIMENSIONAL CRYSTALS $\quad 2$

1.1 Introduction and motivation 2

1.2 Basic electronic properties of graphene 6

1.2.1 Lattice structure of monolayer graphene 6

1.2.2 Energy dispersion relation in monolayer graphene 8

1.2.3 Band structure of bilayer graphene 13

1.3 Density of states in graphene 17

1.4 Chiral tunnelling and the Klein paradox 18

1.4.1 Chiral tunnelling in monolayer graphene n-p-n junction 19

1.4.2 Chiral tunnelling in bilayer graphene n-p-n junction 21

1.5 Dirac fermions in a magnetic field and anomalous quantum Hall effect $\quad 24$

1.5.1 Landau levels and quantum Hall effect in monolayer graphene 25

1.6 Spin-orbit coupling effects in graphene $\quad 29$

1.6.1 Band structure of monolayer graphene in the presence of $\mathrm{SOC} \quad 31$

1.6.2 Band structure of bilayer graphene in the presence of SOC 33

1.7 Minimum conductivity in graphene $\quad 35$

1.7.1 AC conductivity in clean graphene system 37

1.8 Charge polarizability and RKKY interaction in graphene 42

1.8.1 Charge polarizability in monolayer graphene 44

1.8.2 Charge polarizability in bilayer graphene 46

1.8.3 RKKY interaction 49

1.9 Electrical properties of $\mathrm{MoS}_{2} \quad 50$ 
2 ELECTRONIC PROPERTIES OF BILAYER GRAPHENE RINGS 56

2.1 Energy levels in BLGR using a two-band Hamiltonian 56

2.1.1 Calculating the velocity operator 64

2.2 Comparison between two-band and four-band Hamiltonian in BLGR 66

2.3 BLGR in the presence of a magnetic field 70

2.4 BLGR in the presence of Rashba spin orbit coupling $\quad 74$

2.4.1 Calculating the velocity operator in the presence of Rashba SOC 83

2.5 Experimental realization of graphene rings 84

2.6 Summary and conclusions $\quad 85$

3 EFFECT OF ORBITAL-RASHBA COUPLING IN GRAPHENE

3.1 Introduction and motivation 88

3.1.1 Graphene in a transverse electric and perpendicular magnetic fields 89

3.1.2 Orbital Rashba Effect of on Landau levels 94

3.2 AC transport of monolayer graphene in the presence of an electric field 96

3.3 Rabi oscillations in the presence of an in-plane electric field 102

3.4 Summary and conclusion 108

4 CHIRAL TUNNELING IN BLG: EFFECT OF RASHBA SPIN-ORBIT COUPLING $\quad 110$

4.1 Introduction 110

4.2 Band structure of bilayer graphene in presence of Rashba SOC $\quad 111$

4.2.1 Dimensionless relations 112

4.2.2 Band structure in different regimes 113

4.2.3 Spin polarization 114

$4 \cdot 3 \quad \mathrm{~N}-\mathrm{P}-\mathrm{N}$ junction with SO-SO-SO Interfaces 114

4.3.1 Kinematics of scattering 117

4.3.2 Numerical results for npn junction $\quad 117$

4.4 N-P junction with SO-SO interfaces 125 
4.4.1 Comment on the Klein paradox for a pn junction in multilayer graphene $\quad 127$

4.4.2 Numerical results for $n p$ junction 129

4.5 N-P-N junction with N-SO-N interfaces 131

4.6 Summary and conclusion 134

5 SPIN SUSCEPTIBILITY OF TWO-DIMENSIONAL TRANSITION METAL DICHALCOGENIDES 136

5.1 Introduction and motivation 136

5.2 Details of our theoretical approach $\quad 137$

5.2.1 Model-Hamiltonian description 137

5.2.2 Spin susceptibility for a multi-band system $\quad 141$

5.3 Spin susceptibility of electrons: Extrinsic vs. intrinsic contributions

5.3.1 In-plane spin-susceptibility component $\chi_{x x} \quad 146$

5.3.2 Perpendicular spin-susceptibility component $\chi_{z z} \quad 150$

5.4 Spin susceptibility of holes: In-plane/out-of-plane anisotropy $\quad 151$

5.5 Physical consequences of unusual spin response in the hole-doped case $\quad 155$

5.5.1 RKKY interaction and mean-field magetism $\quad 155$

5.5.2 Pauli paramagnetism and effective g-factor $\quad 159$

5.6 Summary and Conclusions 159

6 OVERVIEW 162

6.1 Electronic properties of bilayer graphene rings $\quad 162$

6.2 Orbital Rashba effect in monolayer graphene 164

6.3 Chiral tunnelling in the presence of Rashba spin-orbit coupling $\quad 165$

6.4 Spin response of monolayer transition metal dichalocogenides 166

6.5 Publications resulting from this thesis 168

A APPENDIX 170

A.1 Probability current in BLG in the presence of Rashba SOC $\quad 170$ 



\section{ACRONYMS}

oD Zero-Dimensional

1D One-Dimensional

2D Two-Dimensional

${ }_{3} \mathrm{D} \quad$ Three-Dimensional

MLG Monolayer Graphene

BLG Bilayer Graphene

BLGR Bilayer Graphene Ring

MLGR Monolayer Graphene Ring

2DEG Two-Dimensional Electron Gas

SOC Spin Orbit Coupling

HE Hall Effect

QHE Quantum Hall Effect

QSHE Quantum Spin Hall Effect

DFT Density Functional Theory

AC Alternating Current

AB Aharonov-Bohm

DOS Density of State

RKKY Ruderman-Kittel-Kasuya-Yosida 



\section{REVIEW OF ELECTRONIC PROPERTIES OF TWO \\ DIMENSIONAL CRYSTALS}

\section{I.I INTRODUCTION AND MOTIVATION}

Carbon atoms can be arranged in many different ways in nature. For example, one can find pure carbon in nature in the form of diamond or graphite which have very different properties since diamond is insulating and hard while graphite is conducting and soft. The first nano-scale carbon allotrope was fullerene which was first discovered in 1985 (Kroto et al., 1985). It was in 1991 when the structure of carbon nanotubes was first identified (Iijima et al., 1991). Due to their potential application in electronic devices, carbon nanotubes attracted a lot of attention rapidly (Saito et al., 1998). The diameter of these nanotubes is of the order of several nanometers and in 2009, the length of carbon nanotubes reached $18.5 \mathrm{~cm}$ (Wang et al., 2009). Finally in 2004, an experimental group in Manchester University led by Andre Geim and Konstantin Novoselov isolated the two dimensional (2D) allotrope of carbon atoms called graphene (Novoselov et al., 2004). Graphite, another allotrope of carbon, which for example was interesting due to its applications in nuclear experiments, is nothing more than three-dimensional $\left({ }_{3} \mathrm{D}\right)$ stack of graphene layers. For this reason, the electronic properties of graphene have been well known theoretically for many years before 2004. The two-dimensional graphene can be considered as the building block of other carbon allotropes. For example, fullerene is zero-dimensional (oD) wrapped up graphene, or carbon nanotubes are one-dimensional ( (1D) rolled graphenes, see Fig. 1 .

Because of two main reasons, people had always believed that strictly $2 \mathrm{D}$ materials could not exist in nature. On the one hand, Peierls (Peierls, 1923) and Landau 


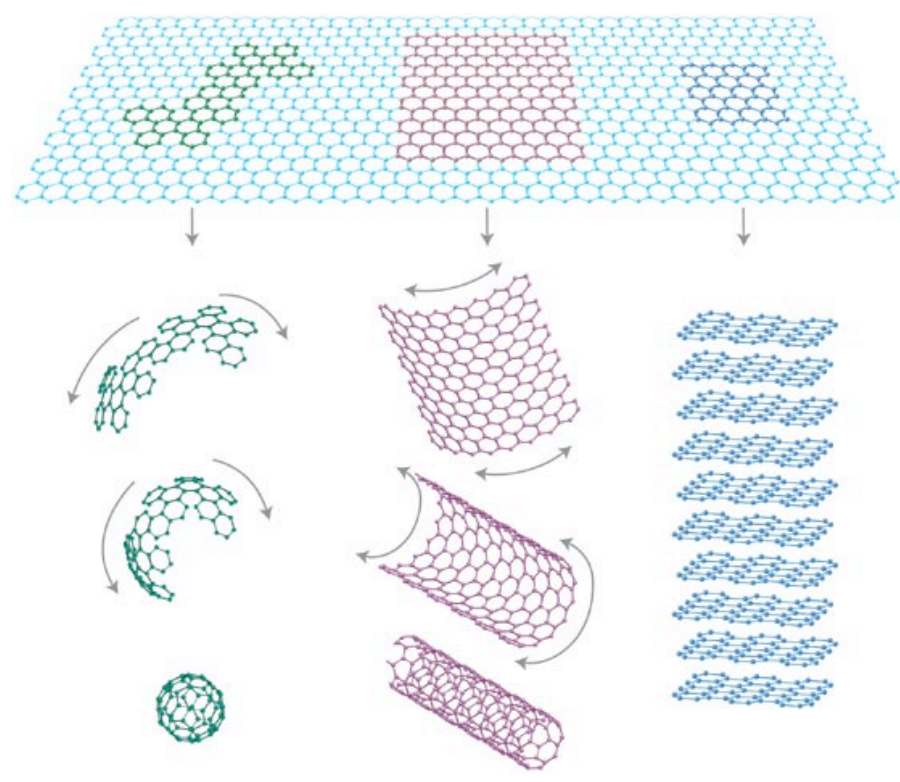

Figure 1: Graphene is the building block of other carbon allotropes. It can be wrapped up to form $\mathrm{oD}$ fullerene or rolled to form $\mathrm{iD}$ carbon nanotubes or stacked into 3D graphite. Copied from Geim and Novoselov (2007).

(Landau, 1937) had shown that 2D structures in the thermodynamic limit are unstable due to thermal fluctuations and eventually melt. On the other hand, due to the Mermin theorem (Mermin, 1968) long range order in 2D crystal structures in the presence of some atomic potentials could not exist. However, graphene layers can exist by having ripples in the third dimension. After years of efforts for isolating monolayer of graphene, it was in 2004-2005 when the first reports of discovering the missing allotrope of carbon, graphene, were submitted by Andre Geim's group from Manchester University-England and Philip Kim's group in Columbia University-United states.

The method which Geim and Novosolov used to successfully isolate the monolayer graphene is called micromechanical cleavage or exfoliation technique which is surprisingly very simple (Novoselov et al., 2005a,b). In this method, layers are peeled off from a bulk graphite by an adhesive tape. In the next step, these flakes are located on a silicon substrate of a certain thickness. Then by using an optical microscope one could search for areas where only one layer of carbon atoms exists. The regions with one layer graphene have different colours from bilayer, 
trilayer and multilayer graphene. Graphene which is obtained from this method is more suitable for research investigation purposes since the samples have a high crystal quality, and their size can be as big as $100 \mu \mathrm{m}$. Chemical exfoliation, thermal decomposition and chemical vapour deposition (CVD) are other methods for isolating graphene which are better for future industrial purposes (Kim et al., 2009; Cooper et al., 2012).

By measuring the stiffness of graphene, it was realized that graphene is the hardest material known (C. Lee et al., 2008). In addition, having a very high electrical mobility even at room temperature, has made graphene a very promising candidate for graphene based electronics. So graphene can be a good candidate for replacing silicon based devices to save Moore's law (Moore, 2006).

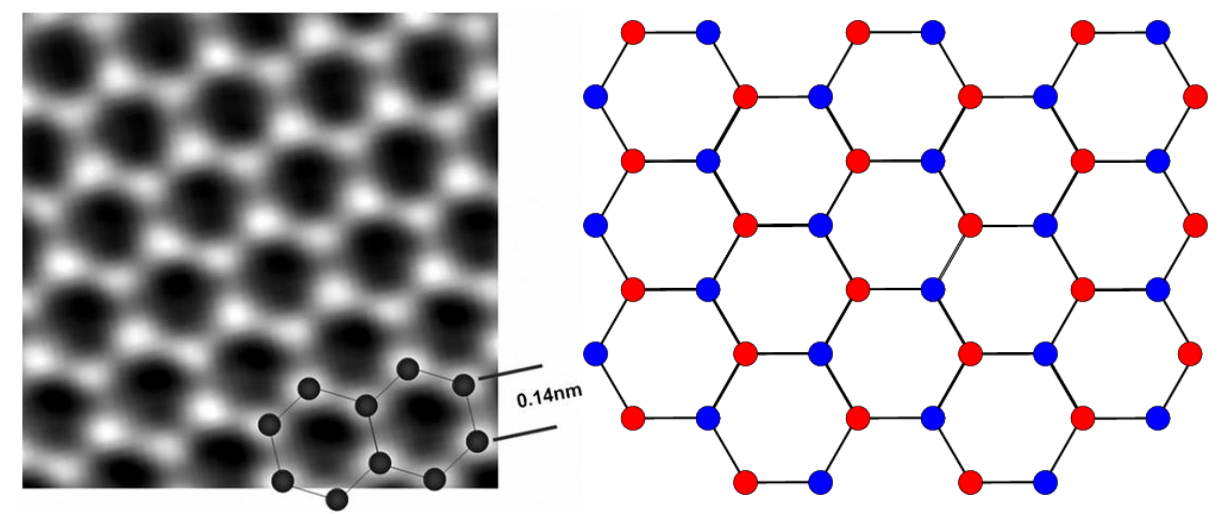

Figure 2: Graphene, Captured by a Transmission Electron Microscope, National Center for Electron Microscopy at Lawrence Berkeley National Laboratory in Berkeley, California (Dato et al., 2009).

Due to the unique crystal symmetry of monolayer graphene, its charge carriers are massless and ruled by the Dirac equation of motion for relativistic particles. Monolayer graphene has a gapless energy spectrum. Two coupled monolayers of graphene, which are stacked as in graphite, is called bilayer graphene (BLG). Charge carriers in bilayer graphene have a parabolic energy spectrum which indicates that these carriers are massive quasiparticles. Bilayer and also multilayer graphene have recently attracted significant attention. Bilayer graphene is more 
convenient to be used in electronic devices since producing a gap in their energy spectrum is easier than in monolayer graphene (McCann and Falko, 2006).

Despite the tremendous potential there are two major obstacles for graphene based applications in electronic devices (Schwierz, 2010). Firstly, pristine graphene is gapless, and inducing a gap, which is of great importance in electronic devices, takes a lot of effort. Secondly, due to the lightness of carbon atoms graphene has very weak spin-orbit coupling (SOC) which makes it difficult for spintronics applications (Kane and Mele, 2005). Hence, the search for other 2D materials which can overcome these two weak points and also maintain the phenomenal characteristics of graphene has been increased (Novoselov et al., 2005b). Monolayer of transition metal dichalcogenides is one of the recent discoveries (Wang et al., 2012), which seems to have promising potentials in electronic and spintronic applications.

$\mathrm{MoS}_{2}$ is a very good example of this class of materials. Bulk $\mathrm{MoS}_{2}$ is a semiconductor with indirect gap, however when it is peeled off to have only one layer it becomes a semiconductor with direct band gap. Monolayer $\mathrm{MoS}_{2}$ has a honeycomb lattice with Mo and $S$ atoms sitting on different sublattices. This arrangement has a broken inversion symmetry which is responsible for having a relatively large $(1.66 \mathrm{eV})$ band gap. Also monolayer $\mathrm{MoS}_{2}$ has a strong SOC, due to $d$ orbitals of heavy transition metals, which makes it a good candidate for spintronic applications (Mak et al., 2010).

The goal of this $\mathrm{PhD}$ project has been to theoretically describe the new electronic and magnetic properties of charge carriers in novel two dimensional systems such as graphene and $\mathrm{MoS}_{2}$, and in particular how to manipulate their electronic transport using external electric and magnetic fields.

The remainder of this thesis is organized as follows. In the rest of the current chapter, we provide an overview of the general electric properties of monolayer and bilayer graphene using a tight-binding description. We also review works on minimal conductivity and Klein tunnelling as two intriguing phenomena in graphene. In this chapter, we also discuss previous works on charge polarizabal- 
ity in monolayer and bilayer graphene. Finally, we review the electronic properties of $\mathrm{MoS}_{2}$.

In Chap. 2, we study the electronic properties of massive Dirac Fermions confined in the ring by applying a bias voltage in bilayer graphene. We present an analytical solution for energy levels in bilayer graphene ring using two-band and four-band Hamiltonians. We also calculate persistent current induced by applying a magnetic flux in the ring. We show how Rashba SOC can affect energy levels and persistent current in BLGR.

In Chap. 3, we try to understand the role of an orbital Rashba-like term proportional to an external in-plane electric field. We have studied the effect of this orbital Rashba effect on Landau levels in graphene in the presence of the inplane electric field. We also calculated the correction to AC transport in monolayer graphene due to the presence of this effect.

In Chap. 4, we investigate the effect of Rashba spin-orbit coupling on chiral tunnelling in bilayer graphene n-p-n and n-p junctions. Finally in Chap. 5, we study spin susceptibility in $\mathrm{MoS}_{2}$ and we show that spin response of monolayer $\mathrm{MoS}_{2}$ has an interesting intermediate behavior between that seen in graphene and an ordinary 2 DEG.

\subsection{BASIC ELECTRONIC PROPERTIES OF GRAPHENE}

\subsubsection{Lattice structure of monolayer graphene}

Graphene has a 2D hexagonal or honeycomb lattice and hence it is not a Bravais lattice and consists of two interpenetrating triangular sublattices, which are shown with different colours in Fig. 3. Vectors $\mathbf{a}_{1}$ and $\mathbf{a}_{2}$ are lattice vectors and given by

$$
\mathbf{a}_{1}=\frac{\mathrm{a}}{2}(3, \sqrt{3}), \quad \mathbf{a}_{2}=\frac{\mathrm{a}}{2}(3,-\sqrt{3}),
$$



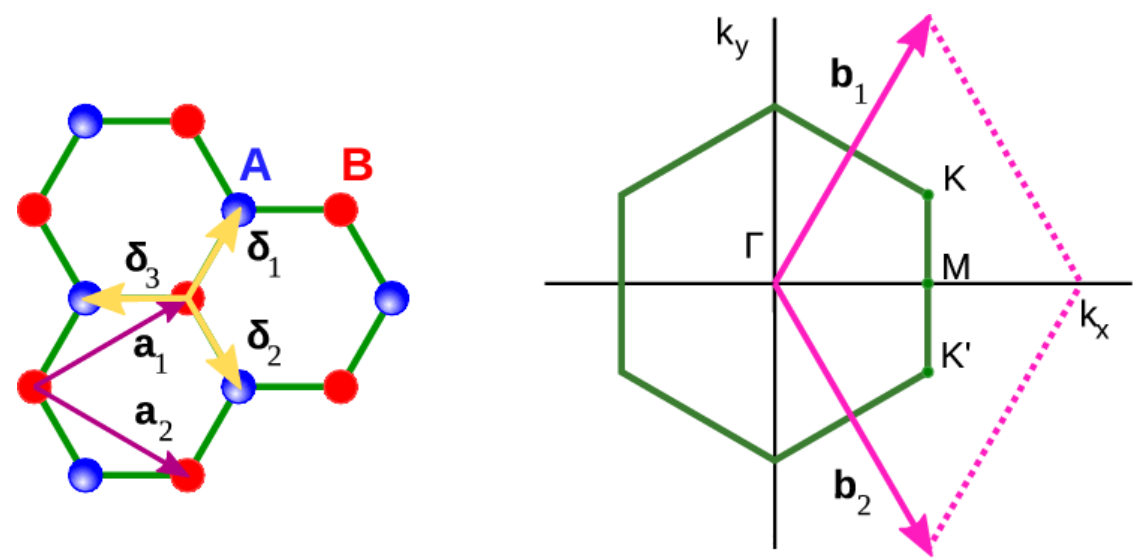

Figure 3: Honeycomb lattice of graphene (left) and its Brillouin zone (right). Graphene lattice composed of two triangular lattices with $\mathbf{a}_{1}$ and $\mathbf{a}_{2}$ indicating unit vectors and $\delta_{i}(i=1,2,3)$ representing nearest-neighbor vectors. Brillouin zone of graphene is also hexagonal with wave vectors $\mathbf{b}_{1}$ and $\mathbf{b}_{2} . \mathrm{K}$ and $\mathrm{K}^{\prime}$ are called Dirac points.

where $a \approx 1.43 \AA$ is the carbon-carbon bond length of graphene. The honeycomb lattice of graphene is a bipartite lattice in which each atom from sublattice $A$ is surrounded by three atoms from sublattice $B$ and vice versa. Hence, there are two atoms in its unit cell. $\delta_{1}, \delta_{2}$ and $\delta_{3}$ are vectors representing the nearest neighbors

$$
\delta_{1}=\frac{a}{2}(1, \sqrt{3}), \quad \delta_{2}=\frac{a}{2}(1,-\sqrt{3}), \quad \delta_{3}=-a(1,0) .
$$

The reciprocal lattice is also a hexagonal lattice (see Fig. 3) with wave vectors $\mathbf{b}_{1}$ and $\mathbf{b}_{2}$ given by

$$
\mathbf{b}_{1}=\frac{2 \pi}{3 a}(1, \sqrt{3}), \quad \mathbf{b}_{2}=\frac{2 \pi}{3 a}(1,-\sqrt{3}) .
$$

High symmetry points $\mathbf{K}, \mathbf{K}^{\prime}$ and $\mathbf{M}$ shown in Fig. 3 are given by wave vectors

$$
\mathbf{K}=\frac{2 \pi}{3 a}\left(1, \frac{1}{\sqrt{3}}\right), \quad \mathbf{K}^{\prime}=\frac{2 \pi}{3 a}\left(1,-\frac{1}{\sqrt{3}}\right), \quad \mathbf{M}=\frac{2 \pi}{3 a}(1,0) .
$$




\subsubsection{Energy dispersion relation in monolayer graphene}

One thing that makes graphene unique from other materials is its dispersion relation (energy-momentum relation) which is linear at low Fermi energies $\left(E_{F}<\right.$ $1 \mathrm{eV})$. In special relativity, the energy of an elementary particle is given by

$$
E=\sqrt{m^{2} c^{4}+p^{2} c^{2}}
$$

where $m$ is the rest mass of the particle, $p$ its momentum and $c$ the speed of light. For ordinary materials, electrons move in their lattices much slower than the speed of light. So the energy-momentum relation in Eq. 2 can be approximated by $E \approx m c^{2}+p^{2} / 2 m$ where $m c^{2}$ is the rest energy, and $p^{2} / 2 m$ is the kinetic energy of the non-relativistic particles.

In condensed matter physics, an electron may travel in a crystal lattice as if its mass is different from the rest mass $(\mathrm{m})$ of the electron. In other words, the electron in the crystal lattice has an effective mass $\left(\mathrm{m}^{*}\right)$ which can be smaller or larger than $m$ depending on the crystal lattice. Due to the special lattice structure of graphene, the effective mass of charge carriers near the Fermi energy is zero $\left(m^{*}=0\right)$. Therefore energy-momentum relation for particles in graphene is given by $E=v_{f} p$ where $v_{f}=1 / 300 c$ is the Fermi velocity. This relation is similar to the relation for ultra-relativistic particles $E=c p$ when $m=0$ in Eq. 2. Due to this linear band structure, electrons behave like massless Fermions. Graphene can therefore be considered as a small lab to investigate relativistic quantum mechanics phenomena such as the Klein paradox (Sec. 1.4).

\subsubsection{Monolayer graphene Hamiltonian in tight binding approximation}

P.R. Wallace in 1947 was the first one who published a paper on the band structure of graphene. He showed the unusual behavior of low energy charge carriers in this material, which behave like massless Dirac Fermions (Wallace, 1947; Slonczewski and Weiss, 1958). In order to calculate the energy dispersion of graphene, we use the second quantization representation of the tight binding model. The 
non-interacting Hamiltonian for $\pi$ electrons of carbon atoms is given by (Wallace, 1947; Neto et al., 2009):

$$
\mathrm{H}_{0}=-\mathrm{t} \sum_{\mathbf{r} \in \mathcal{A}} \sum_{i=1}^{3}\left(\mathrm{a}^{\dagger}(\mathbf{r}) \mathrm{b}\left(\mathbf{r}+\boldsymbol{\delta}_{\mathrm{i}}\right)+\text { c.c. }\right),
$$

where $\mathbf{a}(\mathbf{r})\left(\mathbf{a}^{\dagger}(\mathbf{r})\right)\left\{\mathbf{b}(\mathbf{r})\left(\mathbf{b}^{\dagger}(\mathbf{r})\right)\right\}$ are creation (annihilation) operators of an electron at point $\mathbf{r}$ for sublattices $A\{B\}$, and $t \simeq 2.7 \mathrm{ev}$ is the hopping integral between the two nearest neighbors.

As this system has translational symmetry, it is convenient to write down the Hamiltonian in the momentum space in order to diagonalize it. The Fourier representation of creation and annihilation operators read as

$$
a(\mathbf{k})=\sum_{\mathbf{r} \in A} e^{-i \mathbf{k} \cdot \mathbf{r}} \mathrm{a}(\mathbf{r}), \quad b(\mathbf{k})=\sum_{\mathbf{r} \in B} e^{-i \mathbf{k} \cdot \mathbf{r}} \mathrm{b}(\mathbf{r}),
$$

where $\mathbf{k}$ is the crystal momentum. After some straight-forward algebra, the Hamiltonian in Fourier space can be written as

$$
\mathrm{H}_{0}=\sum_{\mathbf{k}}\left(\Phi(\mathbf{k}) \mathbf{a}^{\dagger}(\mathbf{k}) \mathrm{b}(\mathbf{k})+\text { c.c. }\right),
$$

where $\Phi(\mathbf{k})$ is called the structure factor and is given by

$$
\Phi(\mathbf{k})=-\mathrm{t} \sum_{i} e^{i \mathbf{k} \cdot \delta_{i}}
$$

The eigenvalues of Hamiltonian $\mathrm{H}_{0}$ are $\mathrm{E}= \pm|\Phi(\mathbf{k})|$ where the plus and minus signs are for electron and hole bands respectively. These two bands touch each other at $\mathbf{K}$ and $\mathbf{K}^{\prime}$ points $(|\Phi(\mathbf{k})|=0)$ in the first Brillouin Zone (see Fig. 3), which implies that graphene is a gapless semiconductor. The special $\mathbf{K}$ and $\mathbf{K}^{\prime}$ points are called Dirac points.

In Fig. 4 the dispersion relation obtained from the tight-binding model has been compared with the dispersion relation from density functional theory (DFT) which is an ab-initio approach. It is evident that these two models are in a good agreement especially for low energies close to Dirac points. As is shown in Fig. 4 


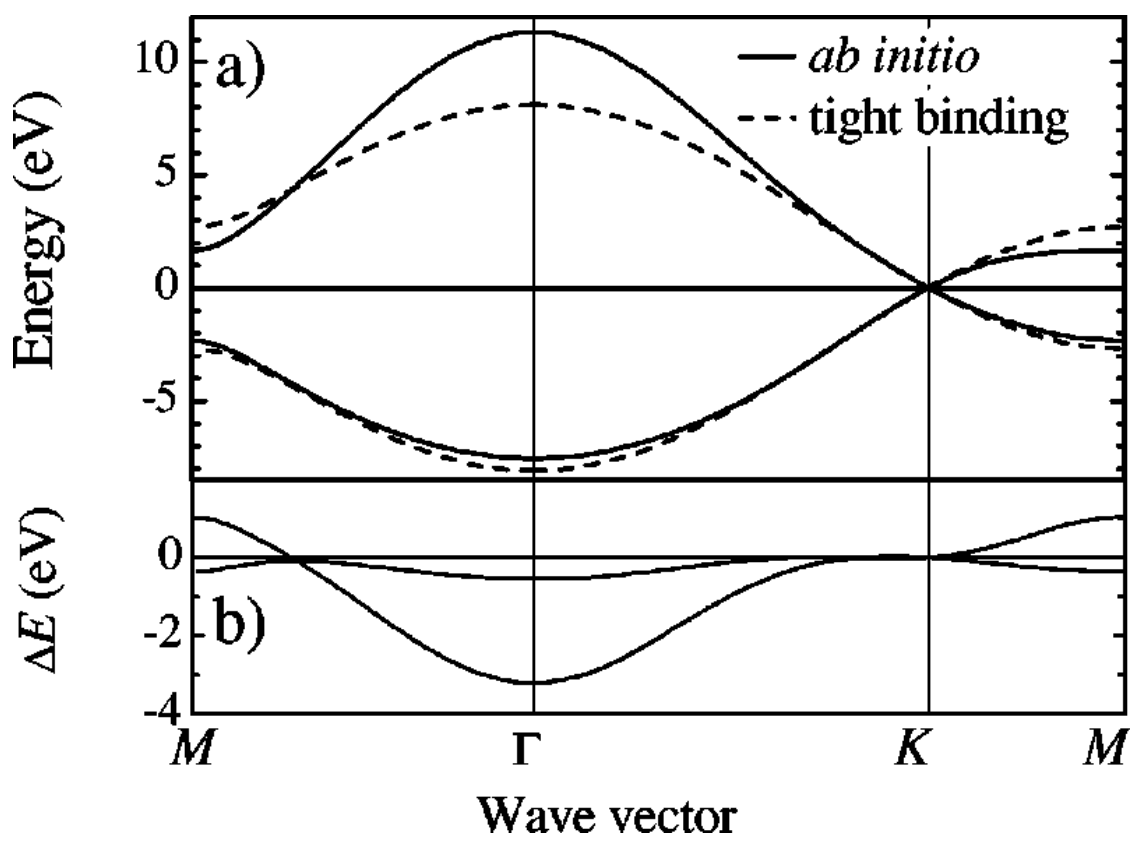

Figure 4: Comparison of graphene energy dispersion obtained from the tight-binding and DFT calculations. This image has been copied from Reich et al. (2002)

for the tight binding approximation, and by considering only the nearest neighbour contribution, the conduction and valence bands are symmetric in the low energy limit.

Since only charge carriers near the Fermi energy can respond to excitation of the system, in condensed matter physics we are interested in low energy studies of the band structure. Here we try to find the effective low energy Hamiltonian for graphene. In order to achieve this goal, we expand the Hamiltonian around its zeros located at Dirac points $\mathbf{K}_{+} \equiv \mathbf{K}$ and $\mathbf{K}_{-} \equiv \mathbf{K}^{\prime}$ as $\mathbf{k}=\mathbf{q}+\mathbf{K}_{ \pm}$with $|\mathbf{q}| \ll\left|\mathbf{K}_{ \pm}\right|$.

We then linearize the Hamiltonian around Dirac points $\mathbf{K}_{ \pm}$

$$
\mathrm{H}_{0}=\int \mathrm{d}^{2} \mathrm{r} \Psi^{\dagger}(\mathbf{r}) \mathcal{H} \Psi(\mathbf{r})
$$

in which $\psi=\left(b_{\mathrm{K}_{+}}^{*}, a_{\mathrm{K}_{+}}^{*}, \mathrm{a}_{\mathrm{K}_{-}}^{*}, \mathrm{~b}_{\mathrm{K}_{-}}^{*}\right)$ is a four component spinor and $\mathcal{H}$ is a $4 \times 4$ matrices given by 


$$
\mathcal{H}=v_{\mathrm{F}}\left(\begin{array}{cccc}
0 & -2 i \partial_{z} & 0 & 0 \\
-2 i \partial_{z}^{*} & 0 & 0 & 0 \\
0 & 0 & 0 & 2 i \partial_{z} \\
0 & 0 & 2 i \partial_{z}^{*} & 0
\end{array}\right)=v_{\mathrm{F}} \boldsymbol{\tau}_{z} \otimes \boldsymbol{\sigma} \cdot \mathbf{q} \text {, }
$$

where $\partial_{z} \equiv \frac{1}{2}\left(\partial_{x}-i \partial_{y}\right)$ and $v_{f}=\frac{3 a t}{2 \hbar} \simeq 10^{6} \mathrm{~m} / \mathrm{s}$ is the Fermi velocity of charge carriers. $\tau_{z}$ is like a Pauli operator acting on degenerate valleys $\mathbf{K}_{ \pm}, \mathbf{q}$ is the wave vector measured from the Dirac points and $\sigma=\left(\sigma_{x}, \sigma_{y}\right)$ is the Pauli matrices acting on sublattice space.

Since the Hamiltonian $\mathcal{H}$ in Eq. 8 is similar to the massless Dirac equation in the Weyl representation, charge carriers in graphene - electrons and holes in the low energy limit $(\mathrm{E}<1 \mathrm{eV})$ behave like massless Dirac Fermions. In a perfect defectless graphene two valleys are decoupled, so instead of the $4 \times 4$ block diagonal Hamiltonian one can use a $2 \times 2$ Hamiltonian for each valley. Thus the two-band Hamiltonian for monolayer graphene for valley $\mathbf{K}$ is

$$
\mathcal{H}_{\mathbf{K}}=\hbar v_{\mathrm{F}} \boldsymbol{\sigma} \cdot \mathbf{q}
$$

and for valley $\mathbf{K}^{\prime}$ reads as

$$
\mathcal{H}_{\mathbf{K}^{\prime}}=\hbar v_{\mathrm{F}} \boldsymbol{\sigma}^{*} \cdot \mathbf{q}
$$

The eigenvalues of this Hamiltonian are given by

$$
E= \pm \hbar v_{\mathrm{F}}|\mathbf{q}|
$$

and it implies that for low energy excitations near the Dirac points the energy dispersion is linear and does not depend on the direction of q. However, if one expands the Hamiltonian $\mathrm{H}_{0}$ up to order $\mathrm{q}^{2}$, the dispersion will depend on the direction of wave vector $\mathbf{q}$. The term of the order $q^{2}$ is called trigonal warping due to its threefold symmetry (Ando and Saito, 1998; Saito et al., 1998).

The eigenstates for the different valleys are

$$
\begin{aligned}
& \psi_{ \pm, K_{+}}=\frac{1}{\sqrt{2}}\left(e^{-i \theta_{q} / 2}, \pm e^{i \theta_{q} / 2}\right)^{\top} e^{i \mathbf{k} . \mathbf{r}} \\
& \psi_{ \pm, K_{-}}=\frac{1}{\sqrt{2}}\left(e^{i \theta_{q} / 2}, \pm e^{-i \theta_{q} / 2}\right)^{\top} e^{i \mathbf{k} . \mathbf{r}}
\end{aligned}
$$


where $\arctan \left(\theta_{\mathrm{q}}\right)=\mathrm{q}_{\mathrm{y}} / \mathrm{q}_{\mathrm{x}}$.

There are several points worth mentioning here. First of all, Dirac fermions in graphene are degenerate. In experiments, the contribution of the two valleys to a measurable quantity usually cancel out each other. Finding a controllable way to break this valley degeneracy opens the door to valleytronics (Rycerz et al., 2007). Second, the eigenstates from different valleys are related by a time reversal symmetry ( $\mathbf{k} \rightarrow-\mathbf{k})$. So if one considers the M point (see Fig. 3 ) being the origin of coordinates in k-space, the time reversal is equivalent to reflection $\left(\left(k_{x}, k_{y}\right) \rightarrow\right.$ $\left.\left(k_{x},-k_{y}\right)\right)$. Third, with a $2 \pi$ change in $\theta$, the phase of the eigenstates changes by $\pi$. The phase change $\pi$ under rotation is a special characteristic of spinors and this phase is usually called the Berry phase (Zhang et al., 2005).

From the similarity of the two Hamiltonians, the Hamiltonian of graphene in the low energy and the Hamiltonian for a relativistic massless Dirac particle, we can redefine some quantities. For example, sublattices A and B in graphene are equivalent to spin-quantum number in Dirac Hamiltonian and based on that they are called pseudo-spin.

Two other concepts that are defined in relativistic electrodynamics are helicity and chirality. Helicity is defined as the projection of the spin component on the direction of motion, and its quantum mechanical operator is given by

$$
\hat{\mathrm{h}}=\frac{1}{2} \frac{\boldsymbol{\sigma} \cdot \mathbf{p}}{|\mathbf{p}|}
$$

The helicity operator commutes with the massless Dirac Hamiltonian, and thus the eigenfunctions in Eq. 12 are also eigenfunctions of $\hat{h}$ with

$$
\begin{aligned}
\hat{h} \psi_{ \pm, K_{+}} & = \pm \frac{1}{2} \psi_{ \pm, K_{+}}, \\
\hat{h} \psi_{ \pm, K_{-}} & =\mp \frac{1}{2} \psi_{ \pm, K_{-}} .
\end{aligned}
$$

Therefore, helicity is a good quantum number for massless Dirac Fermions such as massless neutrinos and charge carriers in graphene. If the spin has the same direction as the particle's motion then, helicity is right-handed, and it is lefthanded if it has the opposite direction. For massless particles, this quantity is 
conserved. Electrons in the conduction band have right-handed helicity whereas in the valance band the helicity of holes is left-handed.

For massless particles like photons or charge carriers in graphene, chirality is the same as helicity. It means that for an external reference, spin (pseudospin) direction and momentum direction of massless particles ruled by Dirac Hamiltonian are always parallel.

\subsubsection{Band structure of bilayer graphene}

BLG consists of two coupled monolayer graphene which are Bernal stacked. Bernal or A-B order is a stacking order in which half of the carbon atoms of the sublattice B from the top layer are located exactly on top of the carbon atoms from sublattice A of the bottom layer. The other carbon atoms from sublattice A from the top layer are located above the middle of the hexagons of the bottom layer. Fig. 5 shows the lattice structure of a Bernal stacked bilayer graphene. Unit cell of bilayer graphene consists of four carbon atoms: $A_{1}$ and $B_{1}$ from top layer (denoted by 1 ) and $A_{2}$ and $B_{2}$ from bottom layer (denoted by 2 ).

\subsubsection{Bilayer graphene Hamiltonian in the tight-binding approximation}

In order to find the Hamiltonian of BLG, we extend the tight-binding model for monolayer graphene. By considering nearest neighbor hopping, the tight-binding Hamiltonian of graphene can be written as (Nilsson et al., 2007; McCann and Koshino, 2013)

$$
\begin{aligned}
H_{0}= & -\gamma_{0} \sum_{<i, j>, l}\left(a_{l i}^{\dagger} b_{l j}+\text { h.c. }\right) \\
& -\gamma_{1} \sum_{<i, j>}\left(a_{2 j}^{\dagger} b_{1 j}+\text { h.c. }\right),
\end{aligned}
$$

where $\gamma_{1} \simeq 0.35 \mathrm{ev}$ is hopping energy between on-top sublattices $A_{1}$ and $B_{2}$ and $\gamma_{0}$ is the nearest neighbor hopping energy in monolayer graphene. The former hopping energy form the dimer bonding in bilayer graphene which leads to 


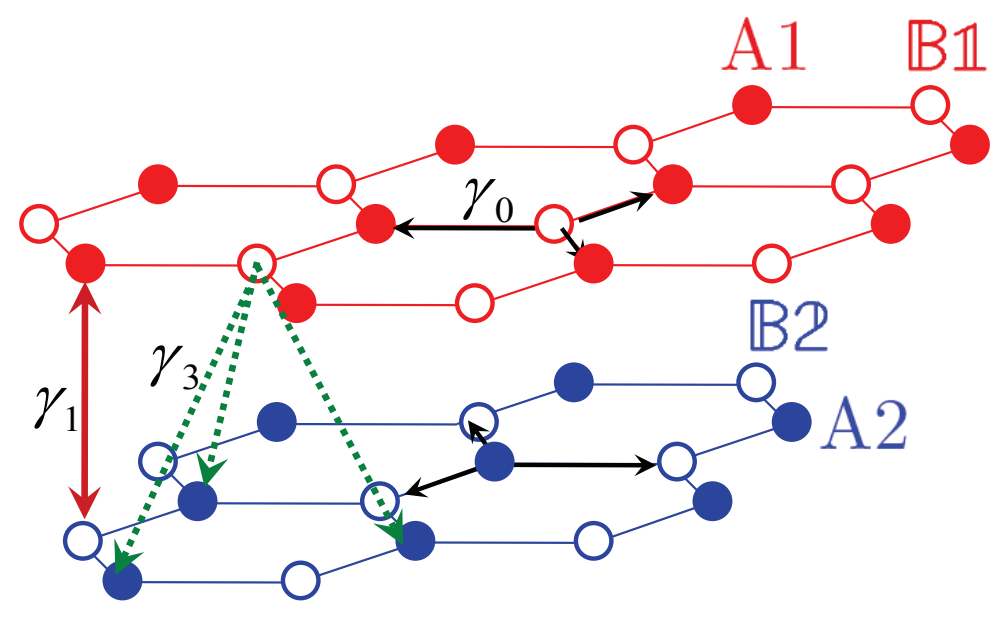

Figure 5: Schematic three-dimensional sketch of Bernal stacked BLG lattice. Indices 1 and 2 denote the different layers. $\gamma_{0}$ is the nearest neighbor intralayer hopping integral, $\gamma_{1}$ denotes the interlayer hopping integral between on-top sublattices $A_{1}$ and $B_{2}$, and $\gamma_{3}$ denotes the interlayer hopping integral between not on-top sublattices $A_{2}$ and $B_{1}$.

having high energy bands. Here we have ignored the hopping energy between the not on-top sublattices $A_{2}$ and $B_{1}$ between two layers i.e. $\gamma_{3}$. Now, by using the Fourier transformation of the annihilation and creation operators in Eq. 4, the tight binding Hamiltonian can be written as $\mathrm{H}_{0}=\sum_{\mathbf{k}} \Psi_{\mathbf{k}}^{\dagger} \mathcal{H} \Psi_{\mathbf{k}}$ where

$$
\mathcal{H}= \pm\left(\begin{array}{cccc}
0 & 0 & 0 & \gamma_{0} \Phi^{*}(\mathbf{k}) \\
0 & 0 & \gamma_{0} \Phi(\mathbf{k}) & 0 \\
0 & \gamma_{0} \Phi^{*}(\mathbf{k}) & 0 & \pm \gamma_{1} \\
\gamma_{0} \Phi(\mathbf{k}) & 0 & \pm \gamma_{1} & 0
\end{array}\right)
$$

and $\Phi(\mathbf{k})$ is defined as in Eq. 6, plus (minus) sign labels valley indices $\mathbf{K}\left(\mathbf{K}^{\prime}\right)$. Eigenvalues of this four band Hamiltonian can be easily obtained by diagonalizing Eq. 16,

$$
E_{ \pm}= \pm\left|\sqrt{\frac{\gamma_{1}^{2}}{4}+\gamma_{0}^{2}|\Phi(\mathbf{k})|^{2}}+\frac{\gamma_{1}}{2}\right|
$$


The band structure of bilayer graphene derived from the four-band Hamiltonian $\mathcal{H}$ is shown in Fig. 6. This band structure has been observed using ARPES technique (Ohta et al., 2006). In the band edge $\left(\mathbf{k}=\mathbf{K}_{ \pm}\right)$, the split bands are separated from the gapless branches by the energy $\gamma_{1}$.

In the low energy limit where $E \ll \gamma_{1}$, we can expand the momentum around the Dirac points as $\mathbf{k}=\mathbf{q}+\mathbf{K}_{ \pm}$with $|\mathbf{q}| \ll\left|\mathbf{K}_{ \pm}\right|$and hence the Hamiltonian can be written as

$$
\mathcal{H} \approx \pm\left(\begin{array}{cccc}
0 & 0 & 0 & \hbar v_{f}\left(q_{x}-i q_{y}\right) \\
0 & 0 & \hbar v_{f}\left(q_{x}+i q_{y}\right) & 0 \\
0 & \hbar v_{f}\left(q_{x}-i q_{y}\right) & 0 & \pm \gamma_{1} \\
\hbar v_{f}\left(q_{x}+i q_{y}\right) & 0 & \pm \gamma_{1} & 0
\end{array}\right)
$$

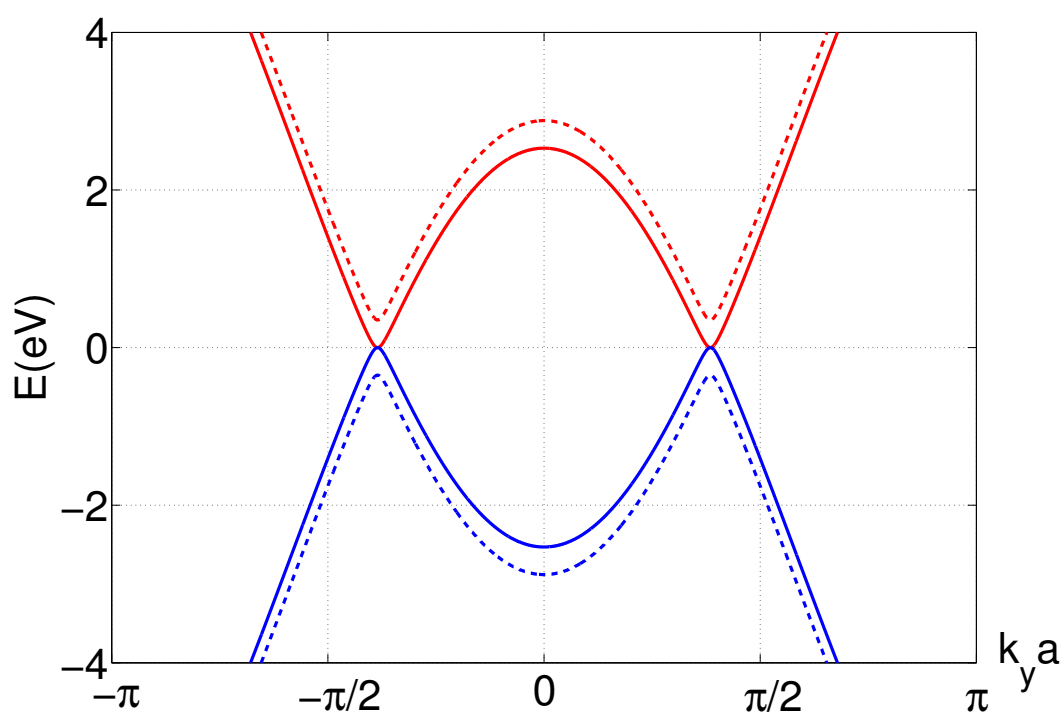

Figure 6: Band structure of bilayer graphene using the four-band Hamiltonian (Eq. 16) versus $k_{y} a$ while $k_{x} a=\frac{2 \pi}{3}$.

The low energy effective Hamiltonian can be written by eliminating high energy states perturbatively (McCann and Falko, 2006): 


$$
\mathcal{H}_{\mathrm{K}_{ \pm}}=\frac{-\hbar^{2}}{2 \mathrm{~m}^{*}}\left(\begin{array}{cc}
0 & \left(q_{x} \mp i q_{y}\right)^{2} \\
\left(q_{x} \pm i q_{y}\right)^{2} & 0
\end{array}\right)
$$

where $\mathrm{m}^{*}$ is the effective mass given by

$$
\mathrm{m}^{*}=\frac{2 \hbar^{2} \gamma_{1}}{3 \mathrm{a}^{2} \gamma_{0}^{2}} .
$$

Diagonalizing Hamiltonian $\mathcal{H}_{\mathrm{K}_{ \pm}}$gives the two parabolic gapless bands as

$$
E_{ \pm}= \pm \frac{\hbar^{2}}{2 m^{*}}\left(q_{x}^{2}+q_{y}^{2}\right)
$$

and eigenfunctions of this Hamiltonian around valley $\mathrm{K}_{+}$are

$$
\psi_{ \pm, K_{+}}=\frac{1}{\sqrt{2}}\left(e^{-i \theta_{q}}, \pm e^{i \theta_{q}}\right)^{T},
$$

and for valley $\mathrm{K}_{-}$are given by

$$
\psi_{ \pm, K_{-}}=\frac{1}{\sqrt{2}}\left(e^{i \theta_{q}}, \pm e^{-i \theta_{q}}\right)^{\top},
$$

where $\theta_{q}=\tan ^{-1}\left(\frac{q_{y}}{q_{x}}\right)$. Note that for bilayer graphene the Berry phase is $2 \pi$. In summary, we can say that near the Fermi energy, charge carriers in monolayer graphene are described by a Dirac-like Hamiltonian $H=v_{f} \vec{\sigma} \cdot \vec{p}$ where $\vec{\sigma}$ is the vector for Pauli matrices and speed of light $c$ is replaced by Fermi velocity $v_{\mathrm{F}}=1 / 300 \mathrm{c}$. This Hamiltonian is a consequence of the peculiar structure of graphene which consists of two equivalent sublattices of carbon atoms. The states near the zero-energy point are composed of the states belonging to these two sublattices. In order to show the contribution of these two sublattices we need an index, like the spin index for spin degree of freedom, so we refer to it as a pseudo-spin. Near the zero-energy points or Dirac points (the points where conduction and valence bands touch each other), charge carriers are chiral which implies that the pseudospin is in or against the direction of their momentum corresponding to helicity eigenvalue plus or minus one respectively. 


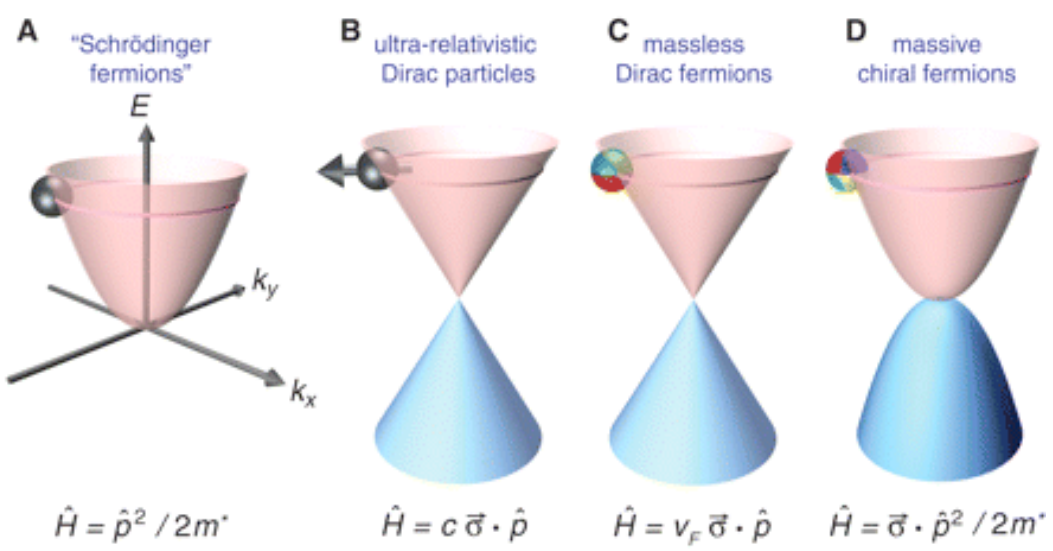

Figure 7: A) Parabolic dispersion relation describing non-relativistic electrons with effective mass $\mathrm{m}^{*}$ and momentum p. B) Massless particles described by the Dirac equation where $c$ is the speed of light and $\vec{\sigma}$ is the vector of Pauli matrices. $C$ ) Charged carriers in graphene behave like massless Dirac fermions described by Dirac Hamiltonian with the speed of light replaced by the Fermi velocity $v_{f}$. D) Band structure of BLG. Charge carriers are massive but still chiral. This image has been taken from Geim (2009).

Linear dispersion of graphene is very different from the parabolic dispersion of low energy charge carriers in conventional semiconductors like GaAs and Silicon. Charged particles in conventional semiconductors are well described by the Schrödinger equation of motion with the Hamiltonian $\hat{\mathrm{H}}=\hat{\mathrm{p}}^{2} / 2 \mathrm{~m}^{*}$. Charge carriers in bilayer graphene behave like a hybrid between ultra-relativistic and non-relativistic electrons: they are chiral excitations with zero rest mass, but their energy-momentum relation is quadratic (see Fig. 7).

\subsection{DENSITY OF STATES IN GRAPHENE}

Density of state (DOS) is defined as the number of states available in each energy level (Ashcroft and Mermin, 1976) and is defined as

$$
\mathrm{D}(\mathrm{E})=\frac{1}{\mathrm{~A}} \sum_{\mathbf{k}} \frac{\partial \mathrm{n}_{\mathbf{k}}}{\partial \mathrm{E}_{\mathbf{k}}}=\frac{1}{\mathrm{~A}} \sum_{\mathbf{k}} \delta\left(\mathrm{E}-\mathrm{E}_{\mathbf{k}}\right), \quad \mathrm{T}=0 .
$$

where $n_{k}$ is the Fermi distribution function and $A$ is the area. 
At zero temperature, the electronic density of states of a conventional two dimensional gas is a constant independent of the charge carrier density and is given by $D(E)=\frac{m^{*}}{\pi \hbar^{2}}$ where $m^{*}$ is the effective mass. However the density of states for graphene with the dispersion relation $E= \pm \hbar v_{F}|k|$ at zero temperature is given by

$$
\mathrm{D}(\mathrm{E})=\frac{g_{s} g_{v}}{2 \pi \hbar^{2} v_{\mathrm{F}}^{2}}|\mathrm{E}|
$$

where $g_{s}$ and $g_{v}$ are spin and valley degeneracies respectively $\left(g_{s}=g_{v}=2\right)$. In contrast to a $2 \mathrm{D}$ electron gas, the DOS of graphene has a linear relation with energy and is no longer a constant. The charge carrier density is given by

$$
n=g_{s} g_{v} \int d \mathbf{k} \Theta\left(E_{F}-E_{k}\right)=\frac{g_{s} g_{v}}{4 \pi} k_{F}^{2}
$$

where $k_{F}$ is the Fermi wave vector. The DOS in Fermi surface is proportional to the second root of the charge carrier density and it goes towards zero for low energy carrier densities.

In bilayer graphene due to the parabolic dispersion relation $E= \pm \hbar^{2} k^{2} / 2 m^{*}$, the DOS is given by

$$
\mathrm{D}(\mathrm{E})=\mathrm{g}_{\mathrm{s}} \mathrm{g}_{v} \frac{\mathrm{m}^{*}}{2 \pi \hbar^{2}}
$$

and like the $2 \mathrm{D}$ electron gas, the DOS in bilayer graphene is constant.

\subsection{CHIRAL TUNNELLING AND THE KLEIN PARADOX}

One of the phenomena predicted in relativistic electrodynamics is the Klein tunnelling due to chiral symmetry. In 1929, by using the Dirac equation, Oskar Klein showed that tunnelling through a potential barrier if the height of the barrier is $V_{0}$ goes to infinity, the reflection tends to zero and an electron always goes through the barrier. Another interesting case is when $m=0$ i.e, massless particles. He 


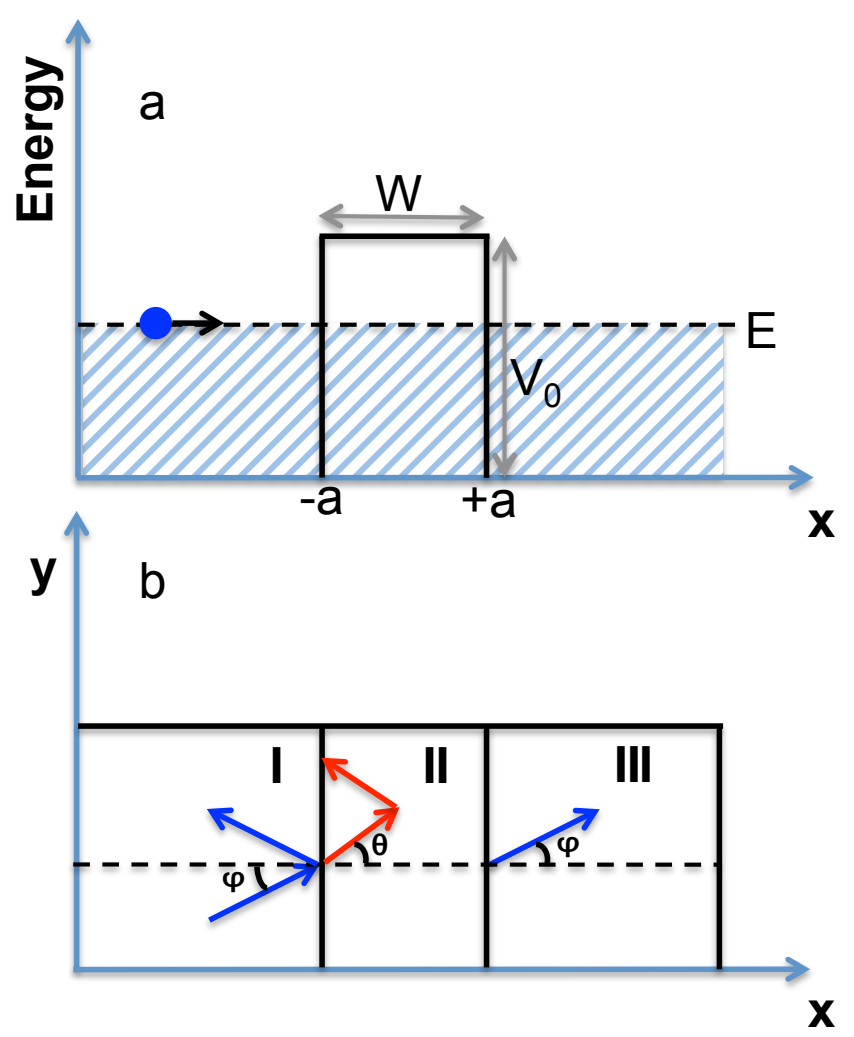

Figure 8: Klein tunnelling in graphene. a) Square potential responsible for scattering of Dirac electrons in graphene. b) Angles of scattering of Dirac electrons in different regions as defined in the text.

showed that for a relativistic massless particle, the transmission coefficient is always one (Calogeracos and Dombey, 1999). Since charge carriers in graphene act like massless Dirac Fermions, graphene can be an appropriate system to observe the effect predicted in high energy physics. Here we briefly study chiral tunnelling of particles through a square barrier in monolayer and bilayer graphene.

\subsubsection{Chiral tunnelling in monolayer graphene $n-p-n$ junction}

For a square potential shown in Fig. 8, we can write the wave function for the three different regions. Since there is translational symmetry in $y$ direction, $k_{y}$ is 
conserved throughout the scattering process. So in region one (denoted by I) the wave function is given by

$$
\psi_{I}(\mathbf{r})=\frac{1}{\sqrt{2}}\left(\begin{array}{c}
1 \\
s e^{i \phi}
\end{array}\right) e^{i\left(k_{x} x+k_{y} y\right)}+\frac{r}{\sqrt{2}}\left(\begin{array}{c}
1 \\
s e^{i(\pi-\phi)}
\end{array}\right) e^{i\left(-k_{x} x+k_{y} y\right)},
$$

where we defined $k_{y}=k_{F} \sin (\phi)$ and $k_{x}=k_{F} \cos (\phi)$ in which $k_{F}$ is the Fermi wave vector and $\phi$ is the incidence angle and $s=\operatorname{sgn}(E)$. In region two (denoted by II), the wave function is

$$
\psi_{I I}(\mathbf{r})=\frac{a}{\sqrt{2}}\left(\begin{array}{c}
1 \\
s^{\prime} e^{i \theta}
\end{array}\right) e^{i\left(q_{x} x+k_{y} y\right)}+\frac{b}{\sqrt{2}}\left(\begin{array}{c}
1 \\
s^{\prime} e^{i(\pi-\theta)}
\end{array}\right) e^{i\left(-q_{x} x+k_{y} y\right)},
$$

where $\theta=\tan ^{-1}\left(\frac{k_{y}}{q_{x}}\right), q_{x}=\sqrt{\left(V_{0}-E\right)^{2} / v_{f}^{2}-k_{y}^{2}}$ and $s^{\prime}=\operatorname{sgn}\left(E-V_{0}\right)$ and finally for the third region (denoted by III) we have

$$
\psi_{\text {III }}(\mathbf{r})=\frac{t}{\sqrt{2}}\left(\begin{array}{c}
1 \\
s e^{i \phi}
\end{array}\right) e^{i\left(k_{x} x+k_{y} y\right)}
$$

The coefficients $r, a, b$ and $t$ can be obtained from the continuity of the wave function across the interface of different regions. Eventually, the transmission probability of the incident electrons is obtained as (Katsnelson et al., 2006)

$$
\mathrm{T}(\phi)=\mathrm{tt}^{*}=\frac{\cos ^{2} \phi \cos ^{2} \theta}{\left[\cos \left(D q_{x}\right) \cos \phi \cos \theta\right]^{2}+\sin ^{2}\left(D q_{x}\right)\left(1-s s^{\prime} \sin \phi \sin \theta\right)^{2}},
$$

In the limit of $\left|V_{0}\right|>>|E|$ the transmission probability can be shown in a simpler form as

$$
\mathrm{T}(\phi)=\frac{\cos ^{2} \phi}{1-\cos ^{2}\left(\mathrm{Dq} \mathrm{q}_{\mathrm{x}}\right) \sin ^{2} \phi} .
$$

It can be seen that when $D q_{x}=n \pi$ in which $n$ is an integer, the transmission probability regardless of the angle of incidence, is always one, i.e. $T(\phi)=1$. On 
the other hand in the normal incidence case $(\phi=0)$, the transmission is always perfect $\mathrm{T}(0)=1$, which is in contrast to the case of non-relativistic particles, see Fig. 9. Since helicity $h=\sigma \cdot p$ is a good quantum number and is conserved during the scattering from the barrier, the reflection coefficient is always zero, or one can simply say that the potential barrier is not able to invert the group velocity of the relativistic massless particles.

\subsubsection{Chiral tunnelling in bilayer graphene $n-p-n$ junction}

Now we study the tunnelling of massive chiral fermions through a barrier in a bilayer graphene. To describe the charge carrier in bilayer graphene, we use the low energy effective Hamiltonian:

$$
\mathcal{H}_{\mathbf{K}_{ \pm}}=\frac{-\hbar^{2}}{2 m^{*}}\left(\begin{array}{cc}
0 & \left(k_{x} \mp i k_{y}\right)^{2} \\
\left(k_{x} \pm i k_{y}\right)^{2} & 0
\end{array}\right) .
$$

Here, in contrast to monolayer graphene, the energy dispersion relation is parabolic and therefore there are four solutions for the wave vectors of a given energy. Two of them correspond to propagating modes, and the other two are wave vectors of evanescent modes. Having evanescent waves in this system is also different from the Schrödinger and Dirac cases. Since we have translational symmetry in $y$ direction, we can write the solutions as

$$
\Psi(x, y)=\psi(x) e^{i k_{y} y}
$$

where $\psi(x)$ is a spinor. This wave function transforms the eigenvalue relation $\mathcal{H} \psi=\mathrm{E} \psi$ to

$$
\left(\frac{d^{2}}{d x^{2}}-k_{y}^{2}\right) \psi_{i}=\left(\frac{2 m\left(E-V_{0}\right)}{\hbar^{2}}\right) \psi_{i} \equiv k^{4} \psi_{i},
$$

so the two propagating $e^{ \pm i k_{x} x}$ and two evanescent waves $e^{ \pm k_{x} x}$ are given by

$$
\begin{aligned}
k_{x}^{2}+k_{y}^{2} & =\frac{2 m\left|E-V_{0}\right|}{\hbar^{2}}, \\
k_{x}^{2}-k_{y}^{2} & =\frac{2 m\left|E-V_{0}\right|}{\hbar^{2}} .
\end{aligned}
$$


The solutions for Eq. 34 for different regions are given by

$$
\begin{aligned}
\psi_{\mathrm{I}}(\mathbf{r})= & a_{1}\left(\begin{array}{c}
1 \\
s e^{2 i \phi}
\end{array}\right) e^{i k_{x} x}+r\left(\begin{array}{c}
1 \\
s e^{-2 i \phi}
\end{array}\right) e^{-i k_{x} x}+c_{1}\left(\begin{array}{c}
1 \\
-s h_{1}
\end{array}\right) e^{k_{x} x}, \\
\psi_{\text {II }}(\mathbf{r})= & a_{2}\left(\begin{array}{c}
1 \\
s^{\prime} e^{2 i \theta}
\end{array}\right) e^{i q_{x} x}+b_{2}\left(\begin{array}{c}
1 \\
s^{\prime} e^{-2 i \theta}
\end{array}\right) e^{-i q_{x} x} \\
& +c_{2}\left(\begin{array}{c}
1 \\
-s^{\prime} h_{2}
\end{array}\right) e^{\lambda_{x} x}+d_{2}\left(\begin{array}{c}
1 \\
-s^{\prime} / h_{2}
\end{array}\right) e^{-\lambda_{x} x}, \\
\psi_{\text {III }}(\mathbf{r})= & t\left(\begin{array}{c}
1 \\
1 \\
s e^{2 i \phi}
\end{array}\right) e^{i k_{x} x}+d_{3}\left(\begin{array}{c}
-s / h_{1}
\end{array}\right) e^{-k_{x} x},
\end{aligned}
$$

where $r$ and $t$ denote reflection and transition coefficients, $k=\frac{\sqrt{2 m E}}{\hbar}$ and $q=\frac{\sqrt{2 m\left(E-V_{0}\right)}}{\hbar}$ are the wave vectors for different regions of the junction. The angle of incidence is $\phi$, and $\theta$ shows the direction of momentum $q$ inside the barrier. Having translational symmetry implies that $k_{y}=k \sin (\phi)=q_{y}=q \sin (\theta)$, which means transverse momentum is conserved. The wave vectors for evanescent modes are defined as $\kappa_{x}=k \sqrt{1+\sin ^{2} \phi}$ and $\lambda_{x}=q \sqrt{1+\sin ^{2} \theta}$. Furthermore $s=\operatorname{sgn}(E), s^{\prime}=\operatorname{sgn}\left(E-V_{0}\right), h_{1}=\left(\sqrt{1+\sin ^{2} \phi}-\sin \phi\right)^{2}$ and $h_{2}=$ $\left(\sqrt{1+\sin ^{2} \theta}-\sin \theta\right)^{2}$. One can find the unknown coefficients $r$ and $t$ from the continuity of the wave function and its derivative at interfaces $x= \pm a$. By solving the system of equations numerically one can see that the transmission for electrons at normal incidence is zero, however there are still magic angles where the transmission probability is one, see Fig. 9. One can find an analytical solution for the transmission probability $T=t t^{*}$ for the case $V_{0}>E$ (Katsnelson et al., 2006):

$$
\mathrm{T}=\frac{\mathrm{E}}{\mathrm{V}_{0}} \sin ^{2}(2 \phi)
$$

which shows that transmission for normal incidence is forbidden but like the monolayer case there are some magic angles in which the barrier is transparent 
90

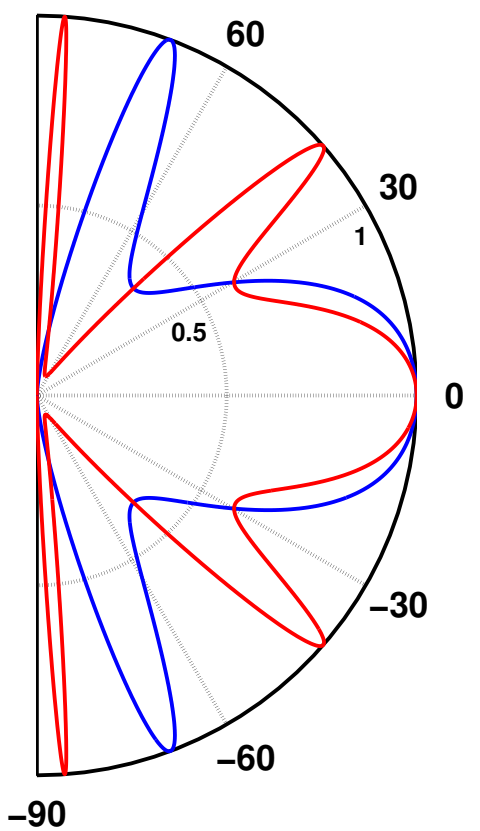

90

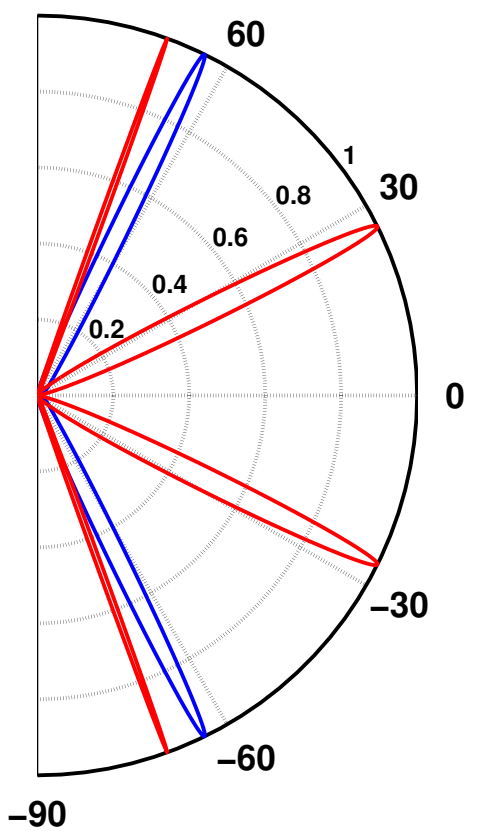

Figure 9: Chiral tunneling in monolayer (left) and bilayer (right) graphene n-p-n junction. In order to compare the results with Katsnelson et al. (2006) the height of the barrier for monolayer is chosen to be $200 \mathrm{meV}$ (red) and $285 \mathrm{meV}$ (blue) and for bilayer graphene $50 \mathrm{meV}$ (red) and $100 \mathrm{meV}$ (blue). The width of the barrier is chosen $\mathrm{W}=100 \mathrm{~nm}$ and the energy of the incident electrons for monolayer graphene is $E_{f}=80 \mathrm{meV}$, and for bilayer graphene it is $E_{f}=17 \mathrm{meV}$. 
$(T=1)$. It is also possible to find an analytical expression for the normal incidence case $(\phi=\theta=0)$ that gives the transmission coefficient as

$$
t=\frac{4 i k q e^{2 i k a}}{(q+i k)^{2} e^{-2 q a}-(q-i k)^{2} e^{2 q a}}
$$

It is easy to see that the transmission probability goes to zero when the width of the barrier tends to infinity (Katsnelson et al., 2006). Signature of chiral tunnelling has been observed in graphene (Young and Kim, 2009).

\subsection{DIRAC FERMIONS IN A MAGNETIC FIELD AND ANOMALOUS QUANTUM HALL EFFECT}

In this section we study the behavior of Dirac Fermions of graphene in the presence of a perpendicular magnetic field (Novoselov et al., 2005a; Neto et al., 2009; Novoselov et al., 2007). The quantum Hall effect (QHE), which is the quantum equivalent version of ordinary Hall effect (HE), can be seen in 2D systems at low temperature and in the presence of a high magnetic field (Prange and Girvin, 1987; Laughlin, 1981). The Hall conductivity is given by

$$
\sigma_{x y}=v \frac{e^{2}}{h}, \quad R_{K}=\frac{h}{e^{2}}=25812.807557 \Omega
$$

where $e$ is the electron charge, $h$ the Planck constant, $v$ the filling factor and $R_{K}$ is the von Klitzing constant. Note that in the ordinary quantum Hall, the spin degeneracy is broken. Depending on the filling factor being integer $v=$ $1,2,3, \ldots$ or fractional $v=1 / 3,1 / 5,5 / 2,12 / 5, \ldots$ the quantum Hall effect is called integer quantum Hall or fractional quantum Hall effect respectively. Integer QHE happens for non-interacting single particle systems, whereas fractional QHE is more complicated and has the root in electron-electron interactions. In 2009 the fractional QHE with filling factor $v=1 / 3$ was observed for the first time in graphene (Bolotin et al., 2009; Du et al., 2009). 


\subsubsection{Landau levels and quantum Hall effect in monolayer graphene}

One of the intriguing characteristics of graphene which attracted a lot of attention among scientists since the early days of its discovery was observing the anomalous QHE even at room temperature. In order to understand this effect, we recalculate the Landau levels in graphene. For a uniform perpendicular magnetic field $B$ we can use the Landau gauge $A=(-B y, 0)$ in order to simplify the calculation. So by replacing $p \rightarrow p+e A$, Dirac equation can written as

$$
\nu_{\mathrm{f}} \sigma .(-i \nabla+e A) \psi(\mathrm{r})=\mathrm{E} \psi(\mathrm{r})
$$

Since the $K$ and $K^{\prime}$ valleys are decoupled we first find the solution for valley $K$. So Eq. 40 gives

$$
v_{F} \sigma_{x}\left(p_{x}-e y B\right)\left(\begin{array}{c}
\Phi_{A}^{K} \\
\Phi_{B}^{K}
\end{array}\right)+v_{F} \sigma_{y} p_{y}\left(\begin{array}{c}
\Phi_{A}^{K} \\
\Phi_{B}^{K}
\end{array}\right)=E\left(\begin{array}{c}
\Phi_{A}^{K} \\
\Phi_{B}^{K}
\end{array}\right),
$$

where $\Phi_{A(B)}^{K}$ is the envelope function of $A(B)$ sublattice for valley $K$. This relation can be written as two coupled differential equations as

$$
\begin{aligned}
& v_{\mathrm{F}}\left(p_{x}-e y B\right) \Phi_{B}^{K}-i v_{F} p_{y} \Phi_{B}^{K}=E \Phi_{A}^{K}, \\
& v_{F}\left(p_{x}-e y B\right) \Phi_{A}^{K}+i v_{F} p_{y} \Phi_{A}^{K}=E \Phi_{B}^{K} .
\end{aligned}
$$

Inserting one equation into the other yields

$$
\begin{aligned}
& \left(p_{x}-e y B\right)^{2} \Phi_{A}^{K}-e B \hbar \Phi_{A}^{K}+p_{y}^{2} \Phi_{A}^{K}=E^{2} / \nu_{F}^{2} \Phi_{A}^{K}, \\
& \left(p_{x}-e y B\right)^{2} \Phi_{B}^{K}+e B \hbar \Phi_{A}^{K}+p_{y}^{2} \Phi_{B}^{K}=E^{2} / \nu_{F}^{2} \Phi_{B}^{K} .
\end{aligned}
$$

In order to find the energy spectrum, we can for example, rewrite the first relation of Eq. 44 as

$$
\left(\frac{1}{2} \bar{K}\left(y-y_{0}\right)^{2}+\frac{p_{y}^{2}}{2 m}\right) \Phi_{A}^{K}=\frac{1}{2 m}\left(E^{2} / \nu_{F}^{2}+e B \hbar\right) \Phi_{A}^{K},
$$


where $\bar{K}=\frac{e^{2} B^{2}}{m}$ and $y_{0}=\frac{p_{x}}{e B}$. The left hand side of Eq. 45 is similar to a Hamiltonian of a harmonic oscillator with its equilibrium distance located at $y_{0}$. Since the energy levels of a harmonic oscillator are given by $\hbar \omega_{\mathcal{c}}(n+1 / 2)$ with a cyclotron frequency of $\omega_{c}=\sqrt{\frac{\bar{k}}{m}}$, the Landau levels in monolayer graphene must satisfy the relation $E^{2}=2 e \hbar B v_{F}^{2} n$, in which $n=\ldots,-1,0,1, \ldots$ is the Landau level index. Therefore, the Landau levels in graphene are given by

$$
E(n)=\operatorname{sgn}(n) \hbar \omega_{D} \sqrt{|n|},
$$

where $\omega_{D}=v_{F} \sqrt{\frac{2 e B}{\hbar}}$ and sgn is the sign function. From Eq. 46 it is noticeable that the distance between the two successive Landau levels in graphene is $\hbar \omega_{D}(\sqrt{|n|+1}-\sqrt{|n|})$. This is unlike the ordinary $2 \mathrm{D}$ electron gas, in which the spacing between Landau levels is constant. In addition, Landau levels in graphene are proportional to $\sqrt{\mathrm{B}}$. This feature is also in contrast with the nonrelativistic $2 \mathrm{D}$ electron gas in which the cyclotron energy is proportional to $\mathrm{B}$. This scaling difference has a major role in observing QHE in graphene at room temperature (Novoselov et al., 2007).

For instance, for magnetic fields of magnitude $B=45 T$ the energy gap between the lowest two consecutive Landau levels, i.e. $n=0$ and $n= \pm 1$, is $\hbar \omega_{D} \approx 2800 \mathrm{~K}$. This gap is much larger than the thermal energy at room temperature. In addition of the large $\hbar \omega_{D}$, the high mobility of Dirac Fermions even at room temperature and also the possibility of doping graphene up to $10^{13} \mathrm{~cm}^{-2}$ in the lowest Landau level, has made it possible to see the QHE at room temperature for charge carriers in graphene. For magnetic fields of the order $\mathrm{B} \simeq 10 \mathrm{~T}$, the $2 \mathrm{D}$ cyclotron energy is only a few Kelvins, whereas this quantity for the same magnetic field is of the order of $10^{3} \mathrm{~K}$ in graphene. In Fig. 1o this difference can be easily seen.

The eigenfunction of Landau levels for valley $\mathrm{K}$ is given by (Zheng and Ando, 2002)

$$
\Psi_{n, k}^{k}(r)=\frac{a_{n}}{\sqrt{L}} e^{-i k y}\left(\begin{array}{c}
\operatorname{sgn}(n) i^{|n|-1} \Phi_{|n|-1} \\
i^{|n|} \Phi_{|n|}
\end{array}\right),
$$



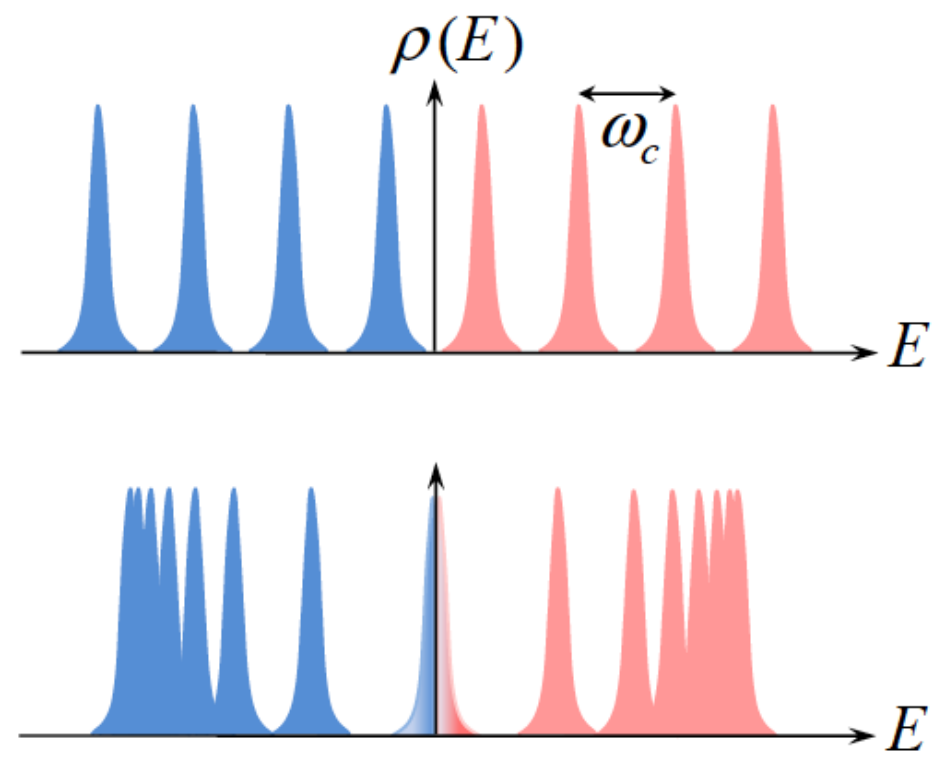

Figure 10: Landau levels for Schrödinger electrons ruled by parabolic dispersion (up) and Dirac electrons (down). This image has been copied from Novoselov et al. (2007).

and for valley $K^{\prime}$ is

$$
\Psi_{n, k}^{K^{\prime}}(r)=\frac{a_{n}}{\sqrt{L}} e^{-i k y}\left(\begin{array}{c}
i^{|n|} \Phi_{|n|} \\
\operatorname{sgn}(n) i^{|n|-1} \Phi_{|n|-1}
\end{array}\right),
$$

with

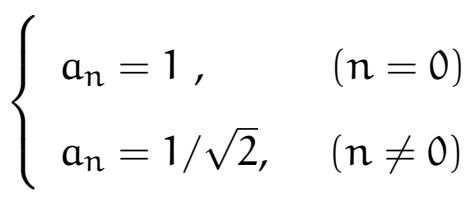

and

$$
\Phi_{|\mathfrak{n}|}=\frac{1}{\sqrt{2^{|n|}|n| ! \sqrt{\pi} l_{B}}} \exp \left(\frac{-1}{2}\left(\frac{x-l_{B}^{2} k}{l_{B}}\right)^{2}\right) H_{|n|}\left(\frac{x-l_{B}^{2} k}{l_{B}}\right),
$$

where $l_{B}=\sqrt{\hbar / e B}$ is the magnetic length scale and $H_{n}(x)$ are the Hermite polynomials. Note that for $n=0$ only one sublattice is occupied.

At first glance it seems that the Hall conductivity in graphene for electrons(+) and for holes(-) by considering spin degeneracy $\mathrm{g}_{\mathrm{s}}=2$ and valley degeneracy $\mathrm{g}_{v}=2$ is $\sigma_{x y}= \pm 4 \mathrm{ne}^{2} / \mathrm{h}$, however as it is depicted in Fig. 11 the Hall conductivity reads as 


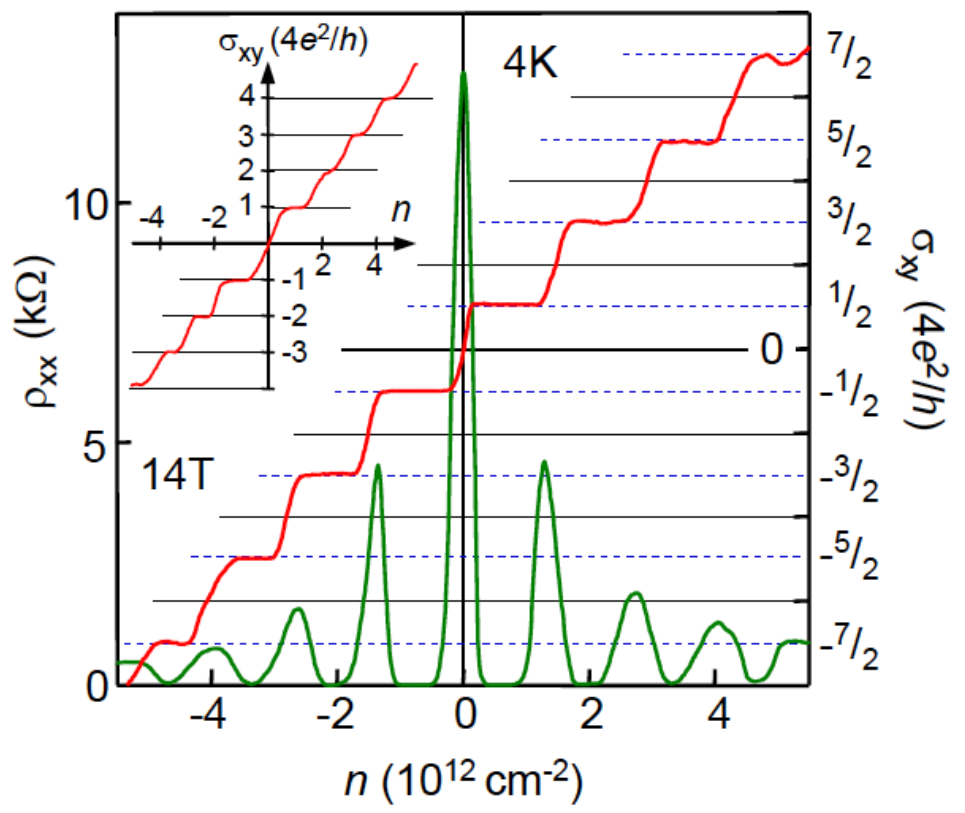

Figure 11: Anomalous quantum Hall effect versus density of charge carriers in graphene in temperature $t=4 \mathrm{~K}$ and magnetic field $B=14 \mathrm{~T}$. Copied from Novoselov et al. (2005a).

$$
\sigma_{x y}= \pm 4(n+1 / 2) e^{2} / h
$$

This difference is rooted in the fact that the zeroth Landau state is shared equally between electrons and holes (Eq. 47). In a neutral graphene (undoped graphene) this state is half filled, thus the contribution of this state in QHE is halved between electrons and holes and by considering a degeneracy factor 4 from spin and valley degrees of freedom, the zeroth Landau level can be considered as two distinct levels for electron doped and the same for hole doped.

For bilayer graphene, Landau levels are given by

$$
E(n)=\operatorname{sgn}(n) \hbar \omega_{c} \sqrt{|n|(|n|-1)},
$$

where $\omega_{c}=e B / m^{*}$. In bilayer graphene, like in the $2 \mathrm{D}$ electron gas, the Landau levels are linear in B. However, unlike the $2 \mathrm{D}$ case, there is an additional zeroenergy level. 


\subsection{SPIN-ORBIT COUPLING EFFECTS IN GRAPHENE}

There are two types of spin-orbit interactions known in graphene. First, intrinsic spin-orbit coupling (intrinsic SOC) which comes from intra-atomic spin-orbit interaction of the carbon atoms in graphene. Second, extrinsic spin-orbit coupling or Rashba SOC due to inversion symmetry breaking of the graphene lattice. Rashba SOC can have different sources such as substrate effects, impurity adatoms or external electric fields like gate voltages.

As we discussed before, pristine graphene has a gapless conical dispersion relation. However, Slonczewski and Weiss (1958); Kane and Mele (2005) showed that this ideal spectrum changes when intrinsic SOC effect is considered in graphene. They showed that intrinsic SOC can induce a gap (intrinsic gap) in graphene equivalent to giving mass to particles, transforms graphene's linear dispersion to a parabolic one. This is, of course, a very ironic situation since SOC is a relativistic effect, but it can destroy the relativistic features of graphene dispersion. Kane and Mele (2005) also showed that SOC effects can bring graphene into the class of quantum spin Hall systems (Kane and Mele, 2005).

Kane and Mele used first-order degenerate perturbation theory and estimated the size of the intrinsic gap about $2 \Delta_{\mathrm{SO}} \sim 2.4 \mathrm{~K}(200 \mu \mathrm{eV})$. They also estimated the strength of Rashba SOC for a perpendicular electric field of the size $E_{z}=$ $50 \mathrm{~V} / 300 \mathrm{~nm}$ to be about $\lambda_{R} \sim 0.5 \mathrm{mK}$. So by their calculations it was possible in experimentally accessible temperatures to have $\Delta_{S O}>\lambda_{R}$, which is the main condition in which quantum spin Hall effect (QSHE) can be realized in graphene (Kane and Mele, 2005).

Later by the use of a tight-binding model and second-order perturbation theory it was shown that Kane and Mele over(under)-estimated the values of $\Delta_{\mathrm{SO}}\left(\lambda_{R}\right)$ by two order of magnitude (Min et al., 2006). So QSHE could be seen in graphene at temperatures less than $0.02 \mathrm{~K}$ (still accessible) and not at $2 \mathrm{~K}$. However, in their tight-binding model, Min et al. (2006) did not consider hoppings and SOC interactions involving $\mathbf{d}$ and higher orbitals, which is very important to get a sizable gap. 
Using group theory, Slonczewski and Weiss (1958) showed that SOC in d orbitals is of first order, whereas for $\mathbf{p}$ orbitals it is of second order. This was the main motivation of Gmitra et al. (2009) and Konschuh et al. (2010) who showed that first-principle and tight-binding calculations, bringing $\mathbf{d}$ orbitals into account, leads to an increase in $\Delta_{\mathrm{SO}}$ from the predicted value of $1 \mu \mathrm{eV}$ to $24 \mu \mathrm{eV}$.

In 2008, a huge SOC (for SOC any values more than $1 \mathrm{meV}$ is considered as huge) about $13 \mathrm{meV}$ was observed in a quasi freestanding graphene. They applied a different approach to produce their monolayer graphene; that is decoupling graphene from its substrate $\mathrm{Ni}(111)$ by intercalating $\mathrm{Au}$ (Varykhalov et al., 2008).

Rashba and intrinsic SOC have been calculated in bilayer graphene using tightbinding (Guinea, 2010; Gelderen and Smith, 2010) and first-principle calculations (Konschuh et al., 2012). For BLG, there are two subdivision for intrinsic and Rashba SOC, which are interlayer (SO interactions between lattice sites in different layers) and intralayer (SO interactions between lattice sites in same layers) spin-orbit interactions. The contribution of interlayer intrinsic SOC is negligible. In Rashba SOC, the orientation of the applied electric field determines their contribution. It has been argued (Konschuh et al., 2012; Gelderen and Smith, 2010) that intralayer SOC in BLG is dominant, which contrasts the results from previous studies (Guinea, 2010) that interlayer SOC is dominant and can enlarge the SOC strength up to ten times. There are two interesting features of having only intralayer Rashba SOC $\left(\Delta_{S O}=0, \lambda_{R} \neq 0\right)$ : first is the splitting of the Dirac cone, which is similar to what happens in monolayer graphene (Zarea and Sandler, 2009) with one main difference, that is the split points of the Dirac cone in BLG are not identical as they are in MLG (Gelderen and Smith, 2010). Second is, of having a linear dispersion in addition to the parabolic one. Intralayer Rashba SOC does not open up a gap, however it splits the bands with different spin orientations, one spin orientation has a parabolic dispersion, and the opposite one has a linear dispersion. 
If one only considers interlayer Rashba SOC, the result will be simply a modification of the interlayer hopping integral $t_{\perp}$

$$
t_{\perp} \rightarrow t_{\perp} \sqrt{1+\lambda_{R}^{\perp} / t_{\perp}^{2}}
$$

In biased BLG, $\lambda_{\mathrm{R}}^{\perp}$ can flatten the Mexican hat dispersion.

Although intrinsic SOC is very weak in graphene due to lightness of the carbon atoms, however, it can still play a major role in understanding several effects such as spin relaxation and spin transport of charge carriers in graphene, or spin Hall effect in graphene nano-ribbons. The weakness of intrinsic SOC is not always a disadvantage, and it is the main reason of having long spin relaxation length in graphene which is of a great importance in spintronic applications (Zutic et al., 2004).

\subsubsection{Band structure of monolayer graphene in the presence of SOC}

The total Hamiltonian in graphene, by bringing SOC effects into account, reads as

$$
\begin{aligned}
& H=H_{0}+H_{S O}+H_{R}, \\
& H_{0}=\hbar v_{f} \sigma \cdot k, \\
& H_{S O}=\lambda_{S O} \tau \sigma_{z} s_{z \prime} \\
& H_{R}=\lambda_{R}\left(\tau \sigma_{x} s_{y}-\sigma_{y} s_{x}\right)
\end{aligned}
$$

where $\mathrm{H}_{S O}$ and $\mathrm{H}_{\mathrm{R}}$ are intrinsic and extrinsic (Rashba) SOC respectively and $\lambda_{\mathrm{SO}}$ and $\lambda_{\mathrm{R}}$ show their strengths.

The Hamiltonian $H$ can be written using $\left(\psi_{A^{\prime}}^{\uparrow}, \psi_{B}^{\uparrow}, \psi_{A}^{\downarrow}, \psi_{B}^{\downarrow}\right)$ as the basis :

$$
H=\left(\begin{array}{cccc}
\lambda_{S O} & \hbar v_{f}\left(k_{x}-i k_{y}\right) & 0 & 0 \\
\hbar v_{f}\left(k_{x}+i k_{y}\right) & -\lambda_{S O} & -2 i \lambda_{R} & 0 \\
0 & 2 i \lambda_{R} & -\lambda_{S O} & \hbar v_{f}\left(k_{x}-i k_{y}\right) \\
0 & 0 & \hbar v_{f}\left(k_{x}+i k_{y}\right) & \lambda_{S O}
\end{array}\right) .
$$



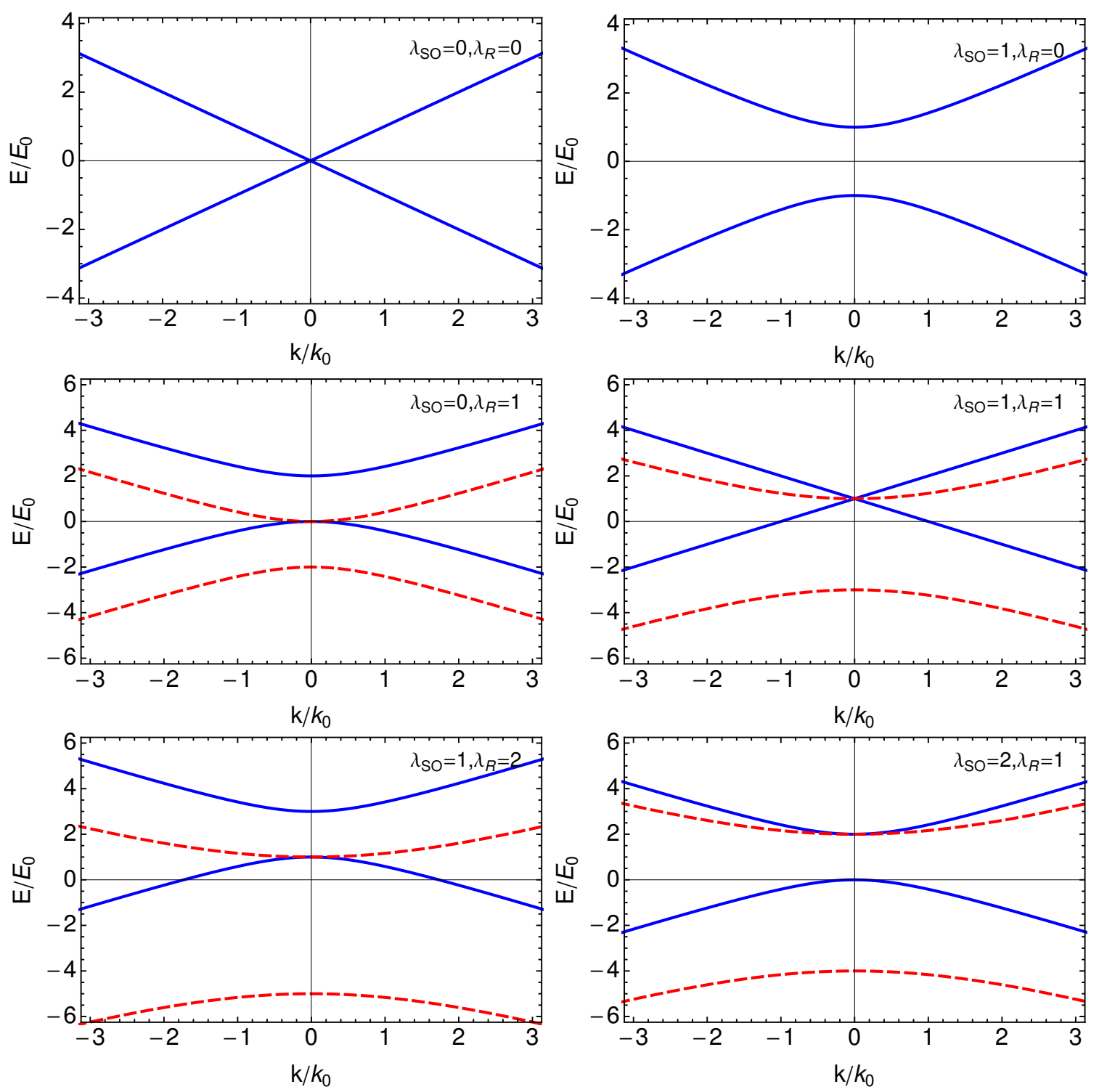

Figure 12: Band structure of graphene in the presence of intrinsic $\left(\lambda_{\mathrm{SO}}\right)$ and extrinsic SOC or Rahba SOC $\left(\lambda_{R}\right)$. Spin- $\uparrow(\downarrow)$ bands are shown as the blue solid (red dashed) curves. The energy scale is $E_{0}=\hbar v_{f} k_{0}$ and $k_{0}=a^{-1}$ where $a$ is graphene lattice constant. 
Here $\lambda_{\text {SO }}$ plays the same role as a mass term and induces a gap of $2 \lambda_{\text {SO }}$ in the spectrum. However, it does not couple the spin up and down states and therefore it does not remove the spin degeneracy. On the other hand, although $\lambda_{R}$ does not break the inversion symmetry and hence it can not generate a band gap, it can lift the spin degeneracy and split the up spin and down spin states by $2 \lambda_{R}$ at the band edge $(k=0)$. The energy eigenstates of this Hamiltonian is given by

$$
E_{\mu s}(k)=s \lambda_{R}+\mu \sqrt{\left(\hbar v_{f} k\right)^{2}+\left(\lambda_{S O}-s \lambda_{R}\right)^{2}}
$$

where $\mu=+1(\mu=-1)$ represents the electron (hole) band and $s=+1(s=-1)$ are spin up (down) band indices. There can be an interesting situation when $\lambda_{R} \geqslant \lambda_{S O}$ which happens for sufficiently high electric fields, and it closes the gap. Dispersion energy of monolayer graphene in the presence of intrinsic and Rashba SOC has been shown in Fig. 12.

\subsubsection{Band structure of bilayer graphene in the presence of SOC}

The low energy effective Hamiltonian for BLG in the presence of only RSOC is given by (Mireles and Schliemann, 2012)

$$
\begin{aligned}
& \mathrm{H}=\mathrm{H}_{0}+\mathrm{H}_{\mathrm{R}}, \\
& \mathrm{H}_{0}=\frac{-1}{2 \mathrm{M}}\left(\pi_{+}^{2} \sigma_{+}+\pi_{-}^{2} \sigma_{-}\right) \\
& \mathrm{H}_{\mathrm{R}}=\frac{i v_{\mathrm{B}}}{\hbar}\left(\pi_{+} \sigma_{+} s_{+}-\pi_{-} \sigma_{-} s_{-}\right) .
\end{aligned}
$$

This Hamiltonian can be written in matrix form using the basis $\left(\psi_{A}^{\uparrow}, \psi_{A}^{\downarrow}, \psi_{B}^{\uparrow}, \psi_{B}^{\downarrow}\right)$ as

$$
H=\left(\begin{array}{cccc}
0 & 0 & -\frac{1}{2 M}\left(k_{x}-i k_{y}\right)^{2} & i v_{B}\left(k_{x}-i k_{y}\right) \\
0 & 0 & 0 & -\frac{1}{2 M}\left(k_{x}-i k_{y}\right)^{2} \\
-\frac{1}{2 M}\left(k_{x}+i k_{y}\right)^{2} & 0 & 0 & 0 \\
-i v_{B}\left(k_{x}+i k_{y}\right) & -\frac{1}{2 M}\left(k_{x}+i k_{y}\right)^{2} & 0 & 0
\end{array}\right),
$$



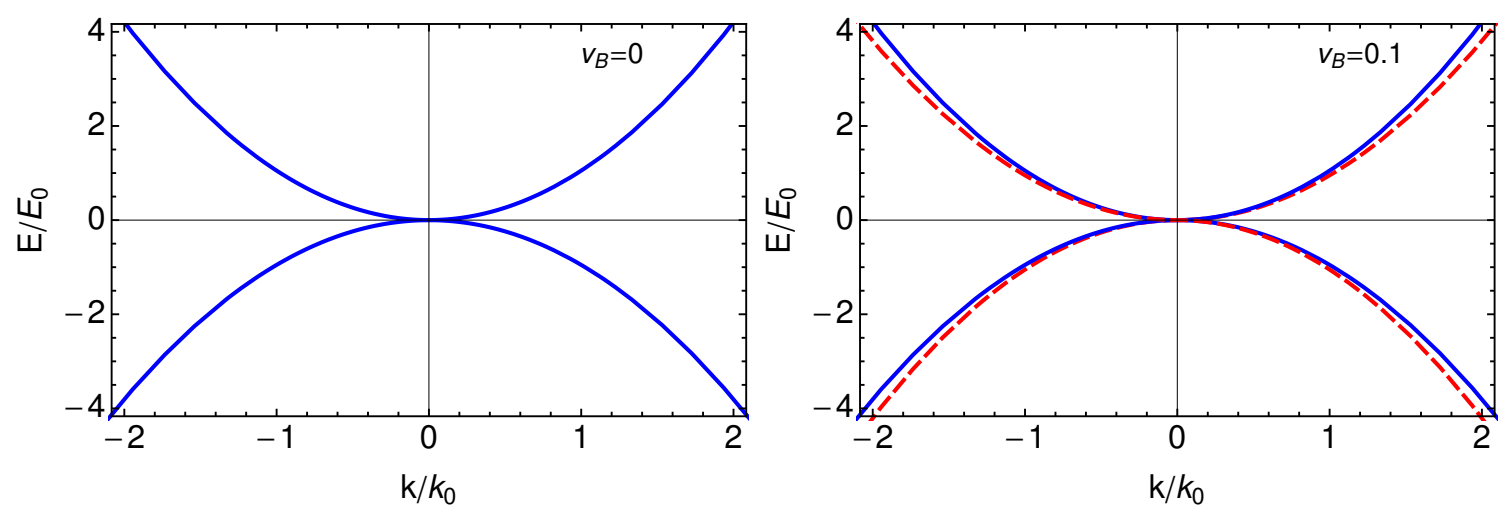

Figure 13: Band structure of bilayer graphene in the absence (left) and in the presence (right) of Rashba spin-orbit coupling $\left(v_{\mathrm{B}}\right)$. Spin- $\uparrow(\downarrow)$ bands are shown by blue solid (red dashed) curves. The energy scale is $E_{0}=\frac{\hbar^{2} k_{0}^{2}}{2 M}$ and $k_{0}=a^{-1}$ where $a$ is graphene lattice constant.

and the eigenvalues and eigenstates read as

$$
\begin{aligned}
& E_{\mu s}(k)=\frac{\mu}{2} k\left(\sqrt{v_{B}^{2}+\frac{\hbar^{4} k^{2}}{M^{2}}}-s v_{B}\right), \\
&\left|\psi_{k s}^{\mu}\right\rangle=\left(\begin{array}{c}
-i \mu s e^{-3 i \phi} \\
-g_{v_{B}}(k) \mu e^{-2 i \phi} \\
i g_{v_{B}}(k) s e^{-i \phi} \\
1
\end{array}\right),
\end{aligned}
$$

where $\mu$ and $s$ with value \pm 1 are band indices indicating the electron/hole branch and spin chirality respectively, and we used the abbreviation $g_{v_{\mathrm{B}}}(k) \equiv$ $\frac{-s v_{B}+\sqrt{v_{B}^{2}+\frac{\hbar^{2} k^{2}}{M^{2}}}}{\frac{\hbar k}{M}}$. In Fig. 13, the dispersion relation of bilayer graphene in the presence of Rashba spin-orbit coupling is shown. In the presence of Rashba spin-orbit coupling, spin polarization $\langle\vec{S}\rangle_{\mu s}=\left\langle\psi_{\mathrm{ks}}^{\mu}|\vec{S}| \psi_{\mathrm{ks}}^{\mu}\right\rangle$ in MLG and BLG in contrast with standard semiconductors is momentum dependent and is given by

$$
\begin{aligned}
& \left\langle S_{x}\right\rangle_{\mu s}=4 s g_{v_{B}}(k) \sin (\phi), \\
& \left\langle S_{y}\right\rangle_{\mu s}=-4 \operatorname{sg}_{v_{B}}(k) \cos (\phi), \\
& \left\langle S_{z}\right\rangle_{\mu s}=0 .
\end{aligned}
$$


The components for the spin polarization for an unbiased bilayer graphene can be written in a compact form by (Mireles and Schliemann, 2012; Rashba, 2009)

$$
\langle\vec{S}\rangle_{\mu s}=\frac{s}{M \sqrt{v_{\mathrm{B}}^{2}+\mathrm{k}^{2} / \mathrm{M}^{2}}}(\hat{\mathbf{z}} \times \mathbf{k}),
$$

which is the same as the case of monolayer graphene with Rashba SOC interaction.

So in unbiased BLG, $\langle\vec{S}\rangle_{\mu s}$ lies in the BLG plane and as long as $v_{B}$ is finite, spin is no longer a good quantum number since $|\langle\vec{S}\rangle| \neq 1$, and in fact $|\langle\vec{S}\rangle|$ has k-dependent oscillations. The orientation of spin polarization is always perpendicular to momentum direction, that is $\vec{S} \cdot \vec{k}=0$. Hence we can define two types of spin polarization with respect to the band index $s$ which are clockwise $s=+1$ and anti-clockwise $s=-1$.

\subsection{MINIMUM CONDUCTIVITY IN GRAPHENE}

Another interesting property in graphene is the existence of conductivity in neutral or undoped graphene. Experiments have shown that the conductivity in graphene decreases linearly by decreasing the carrier concentration or equivalently by decreasing the gate voltage. However, by reducing the carrier density even to zero (close to the Dirac point) the conductivity reaches a finite nonzero value. This universal value is called minimal conductivity, and its value is $\sigma_{\min } \approx 4 e^{2} / \mathrm{h}$. As it can be seen in Fig. 14, this constant value does not change with temperature (Novoselov et al., 2005a; Morozov et al., 2008).

In 1994 Ludwing et.al showed that the conductivity of Dirac fermions using linear response theory, limited frequency at zero temperature and in the absence of impurity is a universal value and is independent of the frequency (Ludwig et al., 1994; Cserti, 2007)

$$
\sigma(\omega)=g_{s} g_{v} \frac{\pi e^{2}}{8 h}
$$




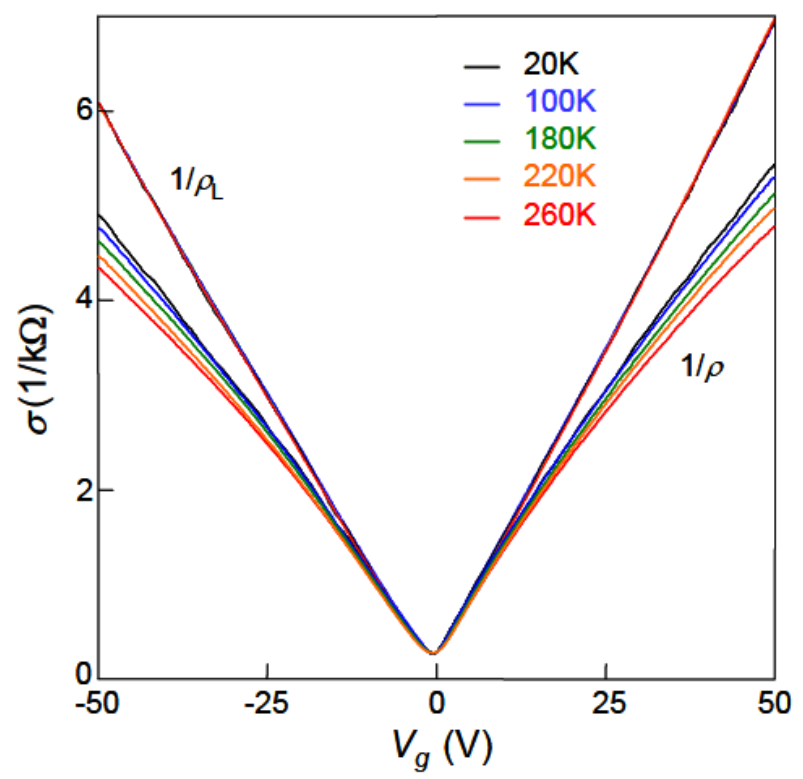

Figure 14: Experimental data of conductivity versus gate voltage for different temperatures. This image has been taken from Morozov et al. (2008).

It also has been shown that, if one first takes the limit of zero frequency before taking the limit of the impurity density to zero, the result would be different (Ludwig et al., 1994; Ziegler, 1997, 1998; Katsnelson et al., 2006)

$$
\sigma_{\min }=g_{s} g_{v} \frac{e^{2}}{\pi h} .
$$

On the other hand, later Ziegler (2006) by using the Kubo formula, showed that the minimal conductivity in graphene is

$$
\sigma_{\min }=g_{s} g_{v} \frac{\pi e^{2}}{4 h}
$$

It is noticeable that there is a difference of $\pi$ between experimental and theoretical values (Ziegler, 2007). In 2007, it was shown experimentally that the predicted value in theory for small graphene flakes with a particular shape is consistent with experiment (Miao et al., 2007). In this experiment, it was shown that for small rectangular graphene samples with a length of $500 \mathrm{~nm}$ and a width of $1 \mu \mathrm{m}$, the minimal conductivity tends to the predicted value in theory. There are different theories about the root of the minimal conductivity in graphene. Klein paradox and existence of evanescent modes (Tworzydlo et al., 2006; Cheianov et al., 
2007b), electron-hole puddles (Tan et al., 2007) and charge impurities (Adam et al., 2007) are a few reasons for the existence of a minimal conductivity.

\subsubsection{AC conductivity in clean graphene system}

In the present section, we calculate the $\mathrm{AC}$ conductivity in monolayer graphene by applying standard linear response theory or the Kubo formalism used in the work of Bernád et al. (2010). In order to calculate the AC conductivity of graphene, the Hamiltonian of the system can be written as,

$$
\begin{aligned}
& H=H_{0}+\delta H \\
& H_{0}=\hbar v\left(\sigma_{x} k_{x}+\sigma_{y} k_{y}\right) \\
& \delta H=\lim _{\alpha \rightarrow 0^{+}} e \vec{E} \cdot \vec{r} e^{-i \omega t+\alpha t}
\end{aligned}
$$

where $\mathrm{H}_{0}$ is the free Hamiltonian of graphene and the perturbation due to the time dependent external electric field is $\delta \mathrm{H}$. Linear response of the system to the perturbation can be found by calculating the time evolution of the density matrix $\rho=\rho_{0}+\delta \rho$, where $\rho_{0}$ is the density matrix of the free Hamiltonian $\mathrm{H}_{0}$ and $\delta \rho$ is due to the perturbation (Madelung, 1978). Since $\rho_{0}$ is time independent, the time evolution of the matrix density $i \hbar \dot{\rho}=[H, \rho]$ reads as

$$
i \hbar \dot{\delta \rho}=\left[\mathrm{H}_{0}, \delta \rho\right]+\left[\delta \mathrm{H}, \rho_{0}\right]
$$

in which terms of the order of $\delta \rho \delta \mathrm{H}$ have been neglected. By writing $\delta \rho$ in Eq. 67 in the interaction picture

$$
\delta \rho=e^{-\frac{i}{\hbar} H_{0} t} \Delta \rho e^{\frac{i}{\hbar} H_{0} t}
$$

we have

$$
i \hbar \dot{\Delta \rho}=e^{\frac{i}{\hbar} H_{0} t}\left[\delta H, \rho_{0}\right] e^{-\frac{i}{\hbar} H_{0} t} .
$$

Now by assuming that the electric field is turned on at time $t=-\infty$ and increases adiabatically to its value at time $t=0$, one can write

$$
\delta \rho(t=0)=\Delta \rho(t=0)=\frac{1}{i \hbar} \int_{-\infty}^{0} d t e^{\frac{i}{\hbar} H_{0} t}\left[\delta H, \rho_{0}\right] e^{-\frac{i}{\hbar} H_{0} t} .
$$


For finding the response of the system to the electric field, we calculate the expectation value of current operator

$$
\begin{aligned}
\langle j\rangle= & \operatorname{Trace}(j \delta \rho) \\
& =\frac{1}{i \hbar} \int_{-\infty}^{0} \operatorname{dtTrace}\left(j e^{\frac{i}{\hbar} H_{0} t}\left[\delta H, \rho_{0}\right] e^{-\frac{i}{\hbar} H_{0} t}\right) .
\end{aligned}
$$

By replacing $\delta \mathrm{H}$ we can write

$$
\begin{aligned}
j_{\mu}= & \frac{1}{i \hbar} \lim _{\alpha \rightarrow 0^{+}} \int_{-\infty}^{0} d t e^{-i \omega t+\alpha t} \\
& \times \operatorname{Trace}\left(j_{\mu} e^{\frac{i}{\hbar} H_{0} t}\left[e E_{\mu} r_{\mu}+e E_{v} r_{v}, \rho_{0}\right]\right) .
\end{aligned}
$$

So the conductivity tensor in $j_{\mu}=\sigma_{\mu \mu} E_{\mu}+\sigma_{\mu \nu} E_{\nu}$ can be written as

$$
\begin{aligned}
& \sigma_{\mu \mu}=\frac{1}{i \hbar} \lim _{\alpha \rightarrow 0^{+}} \int_{-\infty}^{0} d t e^{-i \omega t+\alpha t} \operatorname{Trace}\left(j_{\mu} e^{\frac{i}{\hbar} H_{0} t}\left[\operatorname{er}_{\mu}, \rho_{0}\right] e^{-\frac{i}{\hbar} H_{0} t}\right), \\
& \sigma_{\mu \nu}=\frac{1}{i \hbar} \lim _{\alpha \rightarrow 0^{+}} \int_{-\infty}^{0} d t e^{-i \omega t+\alpha t} \operatorname{Trace}\left(j_{\mu} e^{\frac{i}{\hbar} H_{0} t}\left[\operatorname{er}_{\nu}, \rho_{0}\right] e^{-\frac{i}{\hbar} H_{0} t}\right) .
\end{aligned}
$$

First we calculate $\sigma_{\mu \nu}$

$$
\sigma_{\mu \nu}=\lim _{\alpha \rightarrow 0^{+}} \int_{-\infty}^{0} d t e^{-i \omega t+\alpha t} K_{\mu \nu}
$$

with the Kernel

$$
K_{\mu \nu}=\frac{1}{i \hbar} \operatorname{Trace}\left(j_{\mu} e^{\frac{i}{\hbar} H_{0} t}\left[e r_{v}, \rho_{0}\right] e^{-\frac{i}{\hbar} H_{0} t}\right) .
$$

By using the following relations

$$
\left[r_{v}, \rho_{0}\right]=\rho_{0} \int_{0}^{1 / K_{\beta} T} d \lambda e^{\lambda H_{0}}\left[H_{0}, r_{v}\right] e^{-\lambda H_{0}},
$$

the kernel $K_{\mu \nu}$ can be written as

$$
K_{\mu \nu}=\frac{1}{i \hbar} \operatorname{Trace}\left(j_{\mu} \rho_{0} \int_{0}^{\frac{1}{K_{\beta} T}} \mathrm{~d} \lambda e^{\frac{i}{\hbar} H_{0}(t-i \hbar \lambda)} e\left[H_{0}, r_{\nu}\right] e^{-\frac{i}{\hbar} H_{0}(t-i \hbar \lambda)}\right) \text {. }
$$


By using the eigenstates of the free Hamiltonian $\left(H_{0}\left|E_{m}\right\rangle=E_{m}\left|E_{m}\right\rangle\right)$ to calculate the trace, and also using the relation $j_{\mu}=\frac{-i e}{\hbar}\left[H_{0}, r_{\mu}\right]$ for the current operator it is straight forward to write the kernel as

$$
\begin{aligned}
\mathrm{K}_{\mu v}= & \frac{-e^{2}}{\hbar^{2}} \sum_{n, m} e^{\frac{i}{\hbar}\left(\epsilon_{\mathrm{m}}-\epsilon_{\mathfrak{n}}\right) \mathrm{t}} \frac{{\frac{\left(\epsilon_{\mathfrak{m}}-\epsilon_{\mathfrak{n}}\right)}{k_{\beta T}}}^{\epsilon_{\mathrm{m}}-\epsilon_{\mathrm{n}}}}{\epsilon} \\
& \times\left\langle\epsilon_{\mathrm{n}}\left|\left[\mathrm{H}_{0}, \mathrm{r}_{\mu}\right] \rho_{0}\right| \epsilon_{\mathrm{m}}\right\rangle\left\langle\epsilon_{\mathrm{m}}\left|\left[\mathrm{H}_{0}, \mathrm{r}_{v}\right]\right| \epsilon_{\mathrm{n}}\right\rangle .
\end{aligned}
$$

By applying the definition for the density matrix $\rho_{0}$ as

$$
\begin{aligned}
& \rho_{0}=\mathrm{Z}_{0}^{-1} \exp \left(\frac{-\mathrm{H}_{0}}{\mathrm{~K}_{\beta} \mathrm{T}}\right) \\
& \mathrm{Z}_{0}=\operatorname{Trace}\left(\exp \left(\frac{-\mathrm{H}_{0}}{\mathrm{~K}_{\beta} \mathrm{T}}\right)\right),
\end{aligned}
$$

the kernel $K_{\mu \nu}$ has the following form

$$
\begin{aligned}
K_{\mu v}= & \frac{-e^{2}}{\hbar^{2}} \sum_{n, m} e^{\frac{i}{\hbar}\left(\epsilon_{m}-\epsilon_{n}\right) t} \frac{f\left(\epsilon_{n}\right)-f\left(\epsilon_{m}\right)}{\epsilon_{m}-\epsilon_{n}} \\
& \times\left\langle\epsilon_{n}\left|\left[H_{0}, r_{\mu}\right]\right| \epsilon_{m}\right\rangle\left\langle\epsilon_{m}\left|\left[H_{0}, r_{v}\right]\right| \epsilon_{n}\right\rangle,
\end{aligned}
$$

where $f\left(\epsilon_{n}\right)$ denotes the Fermi function and comes from relation $\rho_{0}\left|\epsilon_{n}\right\rangle=f\left(\epsilon_{n}\right)\left|\epsilon_{n}\right\rangle$ . Thus, the conductivity $\sigma_{\mu \nu}$ reads as

$$
\begin{aligned}
\sigma_{\mu \nu}= & \frac{i e^{2}}{\hbar} \lim _{\alpha \rightarrow 0^{+}} \sum_{n, m} \frac{\left\langle\epsilon_{n}\left|\left[H_{0}, r_{\mu}\right]\right| \epsilon_{m}\right\rangle\left\langle\epsilon_{m}\left|\left[H_{0}, r_{v}\right]\right| \epsilon_{n}\right\rangle}{\left(\epsilon_{m}-\epsilon_{n}-\hbar \omega-i \alpha\right)} \\
& \times \frac{f\left(\epsilon_{n}\right)-f\left(\epsilon_{m}\right)}{\epsilon_{m}-\epsilon_{n}} .
\end{aligned}
$$

By using continuum descriptions of the band structure, the eigenstate of $\mathrm{H}_{0}$ can be written as a product of $|k\rangle$ wave-vector in real space and a spinor $|s\rangle_{k}$

$$
\left|\epsilon_{\mathrm{n}}\right\rangle=|k\rangle \otimes|s\rangle_{\mathrm{k}}
$$

Energy eigenvalues and eigenstates of the Hamiltonian $\mathrm{H}_{0}$ are given by

$$
\begin{array}{r}
\epsilon_{\mathrm{ks}}=s \hbar v_{\mathrm{f}}|\mathrm{k}|, \\
|s\rangle=\frac{1}{\sqrt{2}}\left(\begin{array}{c}
1 \\
s e^{i \theta}
\end{array}\right),
\end{array}
$$


where $s= \pm$ distinguishes between the two (electron and hole) bands. The commutation relations $\left[\mathrm{H}_{0}, \mathrm{r}_{\mu}\right]$ is diagonal in $|k\rangle$ space and we have

$$
\left[\mathrm{H}_{0}, \mathrm{r}_{\mu}\right]|\mathrm{k}\rangle=\mathrm{W}_{\mu}(\mathrm{k})|\mathrm{k}\rangle
$$

So one can write the conductivity $\sigma_{\mu \nu}$ as

$$
\begin{aligned}
\sigma_{\mu \nu}= & \frac{i e^{2}}{\hbar} \lim _{\alpha \rightarrow 0^{+}} \sum_{\sigma, \sigma^{\prime}} \int \frac{d^{2} k}{(2 \pi)^{2}} \frac{\left\langle s\left|W_{\mu}(k)\right| s^{\prime}\right\rangle_{k}\left\langle s^{\prime}\left|W_{v}(k)\right| s\right\rangle_{k}}{\left(\epsilon_{k s^{\prime}}-\epsilon_{k s}-\hbar \omega-i \alpha\right)} \\
& \times \frac{f\left(\epsilon_{k s}\right)-f\left(\epsilon_{k s^{\prime}}\right)}{\epsilon_{k s^{\prime}}-\epsilon_{k s}} .
\end{aligned}
$$

Intraband contribution: First we calculate the conductivity due to intraband transitions, that is transitions occur in the same band $\left(s=s^{\prime}\right)$. So Eq. 85 for an intraband transition reads as

$$
\frac{\sigma_{\mu \nu}^{i n t r a}}{\sigma_{0}}=\frac{\delta(\hbar \omega)}{2} \sum_{s=s^{\prime}} \int d^{2} k W_{\mu}^{s s}(k) W_{v}^{s s}(k) f^{\prime}\left(\epsilon_{k s}\right)
$$

where we have used the scale factor $\sigma_{0}=\frac{g e^{2}}{2 \pi \hbar}$ with $g=4$ being the degeneracy factor (real spin and valley) in graphene. By using $W_{\mu}^{s s}=-i \hbar \nu_{\mathrm{F}} k_{\mu} / k$ we can write

$$
\frac{\sigma_{\mu \nu}^{\text {intra }}}{\sigma_{0}}=\frac{\delta(\hbar \omega)}{2} \int d^{2} k\left(\frac{-\hbar^{2} v_{\mathrm{F}}^{2} k_{\mu} k_{v}}{k^{2}}\right)\left(f^{\prime}\left(\epsilon_{k+}\right)+f^{\prime}\left(\epsilon_{k-}\right)\right) .
$$

By replacing the explicit form of $f^{\prime}\left(\epsilon_{\mathrm{ks}}\right)$ we finally get

$$
\frac{\sigma_{\mu \nu}^{i n t r a}}{\sigma_{0}}=\frac{\delta(\hbar \omega)}{2} \hbar^{2} v_{F}^{2} \int_{0}^{2 \pi} d \theta \frac{k_{\mu} k_{v}}{k^{2}} \int_{0}^{\infty} k d k \frac{\frac{\hbar \nu_{F}}{K_{B} T} \sinh \frac{\hbar \nu_{F} k}{K_{B} T} \sinh \frac{\mu_{F}}{K_{B} T}}{\left(\cosh \frac{\hbar v_{F}}{K_{B} T}+\cosh \frac{\mu_{F}}{K_{B} T}\right)^{2}},
$$

where $\mu_{F}$ denotes the chemical potential, $T$ the temperature and $K_{B}$ the Boltzmann constant. Finally solving the integral over $k$ yields

$$
\frac{\sigma_{\mu \nu}^{\text {intra }}}{\sigma_{0}}=\frac{\delta(\hbar \omega)}{2} \int_{0}^{2 \pi} \mathrm{d} \theta \frac{k_{\mu} k_{v}}{k^{2}} \hbar v_{\mathrm{F}} \mu_{\mathrm{F}}
$$


One can easily see that the intraband contribution for off diagonal elements of the conductivity tensor vanish due to the angular integral in Eq. 89, and its diagonal elements are given by

$$
\frac{\sigma_{x x}^{\text {intra }}}{\sigma_{0}}=\frac{\sigma_{y y}^{\text {intra }}}{\sigma_{0}}=\pi \delta(\hbar \omega) \hbar \nu_{\mathrm{F}} \mu_{\mathrm{F}}
$$

This is the usual dc Drude conductivity, which vanishes when Fermi energy is at neutrality point $\left(\mu_{\mathrm{F}}=0\right)$.

Interband transition: We continue by calculating the contribution to conductivity due to interband transitions $\left(s \neq s^{\prime}\right)$

$$
\begin{aligned}
\frac{\sigma_{\mu \nu}^{i n t e r}}{\sigma_{0}}= & \frac{-1}{2 \hbar \omega} \sum_{s \neq s^{\prime}} \int d^{2} k \delta\left(\hbar \omega-\left(\epsilon_{k s^{\prime}}-\epsilon_{k s}\right)\right) \\
& \times W_{\mu}^{s s^{\prime}}(k) W_{v}^{s^{\prime}} s(k) g\left(\frac{\hbar \omega}{2 k_{\beta} T^{\prime}}, \frac{\mu_{F}}{k_{\beta} T}\right),
\end{aligned}
$$

where we have used the relations

$$
\lim _{\alpha \rightarrow 0^{+}} \frac{1}{i} \frac{1}{\epsilon_{k s^{\prime}}-\epsilon_{k s}-\hbar \omega-i \hbar \alpha}=\pi \delta\left(\epsilon_{k s^{\prime}}-\epsilon_{k s}-\hbar \omega\right),
$$

and

$$
W_{\mu}^{s s^{\prime}}(k)=\left\langle s\left|W_{\mu}(k)\right| s^{\prime}\right\rangle
$$

Also, we introduced the abbreviation

$$
g(\xi, \eta)=\frac{\sinh \xi}{\cosh \xi+\cosh \eta} .
$$

It is straight forward to calculate $W^{s s^{\prime}}(k)$ for the interband transition $\left(s \neq s^{\prime}\right)$ using the eigenstates of the Hamiltonian $\mathrm{H}_{0}$ in Eq. 83

$$
\begin{aligned}
& W_{\mu}^{+-}(k)=<+\left|\left[\mathrm{H}_{0}, \mathrm{r}_{\mu}\right]\right|->=-i \hbar v_{\mathrm{f}}<+\left|\sigma_{\mu}\right|->=\frac{\alpha_{\mu} \hbar v_{\mathrm{f}} \mathrm{k}_{v}}{k}, \\
& W_{\mu}^{-+}(\mathrm{k})=<-\left|\left[\mathrm{H}_{0}, \mathrm{r}_{\mu}\right]\right|+>=-i \hbar v_{\mathrm{f}}<-\left|\sigma_{\mu}\right|+>=-\frac{\alpha_{\mu} \hbar v_{\mathrm{f}} k_{v}}{k},
\end{aligned}
$$

where

$$
\alpha_{\mu}= \begin{cases}-1 & \text { if } \mu \equiv x \\ +1 & \text { if } \mu \equiv y\end{cases}
$$


One can find $\sigma_{x x}$ due to interband transitions as

$$
\begin{aligned}
\frac{\sigma_{x x}^{\text {inter }}}{\sigma_{0}}= & \frac{-1}{2 \hbar \omega} \int d^{2} \mathbf{k} W_{x}^{-+}(k) W_{x}^{+-}(k) g\left(\frac{\hbar \omega}{2 k_{\beta} T^{\prime}}, \frac{\mu_{F}}{k_{\beta} T}\right) \\
& \times\left\{\delta\left(\hbar \omega-\left(\epsilon_{k+}-\epsilon_{k-}\right)\right)+\delta\left(\hbar \omega-\left(\epsilon_{k-}-\epsilon_{k+}\right)\right)\right\},
\end{aligned}
$$

where $\Theta$ is the Heaviside step function.

By using relation $k=\frac{v_{f}}{\omega} k$ to make the equation dimensionless, we reach

$$
\begin{aligned}
\frac{\sigma_{x x}^{\text {inter }}}{\sigma_{0}}= & g\left(\frac{\hbar \omega}{2 k_{\beta} T}, \frac{\mu_{F}}{k_{\beta} T}\right) / 2 \int_{0}^{2 \pi} d \theta \sin ^{2} \theta \int_{0}^{\infty} \kappa d \kappa \\
& \times\{\delta(1-2|\kappa|)+\delta(1+2|\kappa|)\} \Theta(\omega),
\end{aligned}
$$

Finally, solving the integral yields

$$
\frac{\sigma_{x x}^{i n t e r}}{\sigma_{0}}=g\left(\frac{\hbar \omega}{2 k_{\beta} T}, \frac{\mu_{F}}{k_{\beta} T}\right) \frac{\pi}{8} \Theta(\omega) .
$$

By the same calculations one can find that $\sigma_{y y}^{\text {inter }}=\sigma_{x x}^{\text {inter }}$ and $\sigma_{x y}^{\text {inter }}=\sigma_{y x}^{\text {inter }}=0$. Since $g\left(\frac{\hbar \omega}{2 k_{\beta} T}, \frac{\mu_{F}}{k_{\beta} T}\right)$ at the zero temperature limit is one, the interband contribution for the finite $\omega$ is not zero and reaches its universal value $\sigma=\sigma_{0} \pi / 8$. This value agrees with the previous results that showed for finite frequency, at zero temperature, and in the absence of impurity, the minimal conductivity has a universal value and is independent of the frequency (Ludwig et al., 1994; Cserti, 2007; Bernád et al., 2010).

\subsection{CHARGE POLARIZABILITY AND RKKY INTERACTION IN GRAPHENE}

We start this section by finding an explicit form for the charge polarizability function. We start from a charge-charge response function within linear response theory (Giuliani and Vignale, 2005)

$$
\chi^{R}\left(r, r^{\prime}\right)=\frac{-i}{\hbar} \int_{0}^{\infty} d t e^{-\eta t}\left\langle\left[\rho(r, t), \rho\left(r^{\prime}, 0\right)\right]\right\rangle,
$$


where $\rho(r)=\Psi^{\dagger}(r) \Psi(r)$ is the density operator in terms of second-quantized particle creation and annihilation operators $\Psi^{\dagger}$ and $\Psi . \Psi(r)=\left(\Psi^{+}(r), \Psi^{-}(r)\right)$ is a spinor which its elements acting on different valleys. Thus by using energy eigenstates and their annihilation operators $\mathrm{c}_{\mathbf{k} \alpha}^{(\tau, s)}$ we have

$$
\Psi^{\tau}(\mathbf{r})=\sum_{s, \alpha} \int \frac{d^{2} k}{(2 \pi)^{2}} e^{i(\mathbf{k}+\tau K) \mathbf{r}} \psi_{\mathbf{k} \alpha}^{(\tau, s)} c_{\mathbf{k} \alpha}^{(\tau, s)}
$$

Using Eq. 100 one can write the density operator in an explicit form as

$$
\rho(\mathbf{r})=\sum_{\alpha, \beta} \int \frac{d^{2} k}{(2 \pi)^{2}} \int \frac{d^{2} k^{\prime}}{(2 \pi)^{2}} e^{i\left(\mathbf{k}^{\prime}-\mathbf{k}\right) \mathbf{r}}\left(\psi_{\mathbf{k} \alpha}^{\dagger} \psi_{\mathbf{k}^{\prime} \beta}\right) c_{\mathbf{k} \alpha}^{\dagger} c_{\mathbf{k}^{\prime} \beta},
$$

where $\alpha$ and $\beta$ indices include the quantum numbers for spin, valley and sublattice. The time dependent density operator is given by

$$
\rho(\mathbf{r}, \mathbf{t})=\mathrm{e}^{\frac{i}{\hbar} H_{0} t} \rho(\mathbf{r}) \mathrm{e}^{-\frac{i}{\hbar} H_{0} t}=e^{i\left(E_{k \alpha}-E_{\mathbf{k}^{\prime} \beta}\right) \frac{t}{\hbar}} \rho(\mathbf{r}) .
$$

The commutator in Eq. 99 can be written as

$$
\begin{aligned}
{\left[\rho(\mathbf{r}, \mathrm{t}), \rho\left(\mathbf{r}^{\prime}\right)\right]=} & \sum_{\alpha, \beta, \gamma, \delta} \int \frac{d^{2} k}{(2 \pi)^{2}} \int \frac{d^{2} k^{\prime}}{(2 \pi)^{2}} \int \frac{d^{2} k^{\prime \prime}}{(2 \pi)^{2}} \int \frac{d^{2} k^{\prime \prime \prime}}{(2 \pi)^{2}} e^{i\left(\mathbf{k}^{\prime}-\mathbf{k}\right) \mathbf{r}} \mathrm{e}^{i\left(\mathbf{k}^{\prime \prime \prime}-\mathbf{k}^{\prime \prime}\right) \mathbf{r}^{\prime}} \mathrm{e}^{i\left(E_{\mathbf{k} \alpha}-E_{\mathbf{k}^{\prime} \beta}\right) \frac{t}{\hbar}} \\
& \left(\psi_{\mathbf{k} \alpha}^{\dagger} \psi_{\mathbf{k}^{\prime} \beta}\right)\left(\psi_{\mathbf{k}^{\prime \prime} \gamma}^{\dagger} \psi_{\mathbf{k}^{\prime \prime \prime} \delta}\right)\left[c_{\mathbf{k} \alpha}^{\dagger} c_{\mathbf{k}^{\prime} \beta}, c_{\mathbf{k}^{\prime \prime} \gamma}^{\dagger} c_{\mathbf{k}^{\prime \prime \prime} \delta}\right] .
\end{aligned}
$$

The commutator in Eq. 103 is given by

$$
\left[c_{\mathbf{k} \alpha}^{\dagger} c_{\mathbf{k}^{\prime} \beta}, c_{\mathbf{k}^{\prime \prime} \gamma}^{\dagger} c_{\mathbf{k}^{\prime \prime \prime} \delta}\right]=(2 \pi)^{2} \delta^{(2)}\left(\mathbf{k}^{\prime}-\mathbf{k}^{\prime \prime}\right) \delta_{\beta \gamma} c_{\mathbf{k} \alpha}^{\dagger} c_{\mathbf{k}^{\prime \prime \prime} \delta}-(2 \pi)^{2} \delta^{(2)}\left(\mathbf{k}-\mathbf{k}^{\prime \prime \prime}\right) \delta_{\alpha \delta} c_{\mathbf{k}^{\prime \prime} \gamma}^{\dagger} c_{\mathbf{k}^{\prime} \beta},
$$

and its equilibrium average reads as

$$
\left\langle\left[c_{\mathbf{k} \alpha}^{\dagger} c_{\mathbf{k}^{\prime} \beta}, c_{\mathbf{k}^{\prime \prime} \gamma}^{\dagger} c_{\mathbf{k}^{\prime \prime \prime} \delta}\right]\right\rangle=(2 \pi)^{4} \delta_{\beta \gamma} \delta_{\alpha \delta} \delta^{(2)}\left(\mathbf{k}^{\prime}-\mathbf{k}^{\prime \prime}\right) \delta^{(2)}\left(\mathbf{k}-\mathbf{k}^{\prime \prime \prime}\right)\left[n_{\mathrm{F}}\left(E_{\mathbf{k} \alpha}\right)-n_{\mathrm{F}}\left(\mathrm{E}_{\mathbf{k}^{\prime} \beta}\right)\right]
$$

By performing summation and trivial integral we obtain

$$
\begin{aligned}
\left\langle\left[\rho(\mathbf{r}, \mathrm{t}), \rho\left(\mathbf{r}^{\prime}\right)\right]\right\rangle= & \sum_{\alpha, \beta} \int \frac{\mathrm{d}^{2} \mathrm{k}}{(2 \pi)^{2}} \int \frac{\mathrm{d}^{2} k^{\prime}}{(2 \pi)^{2}} \mathrm{e}^{\mathrm{i}\left(\mathbf{k}^{\prime}-\mathbf{k}\right)\left(\mathbf{r}-\mathbf{r}^{\prime}\right)} \mathrm{e}^{i\left(\mathrm{E}_{\mathbf{k} \alpha}-\mathrm{E}_{\mathbf{k}^{\prime} \beta}\right) \frac{\mathrm{t}}{\hbar}} \\
& \times\left(\psi_{\mathbf{k} \alpha}^{\dagger} \psi_{\mathbf{k}^{\prime} \beta}\right)\left(\psi_{\mathbf{k}^{\prime} \beta}^{\dagger} \psi_{\mathbf{k} \alpha}\right)\left[n_{\mathrm{F}}\left(\mathrm{E}_{\mathbf{k} \alpha}\right)-n_{\mathrm{F}}\left(\mathrm{E}_{\mathbf{k}^{\prime} \beta}\right)\right] .
\end{aligned}
$$


By performing variable transformation $\mathbf{k}^{\prime}=\mathbf{q}+\mathbf{k}$ and $\mathbf{R}=\mathbf{r}-\mathbf{r}^{\prime}$ and take the integral over time on the right hand side of Eq. 106, we find the charge-charge correlation function. This function can be written as the Fourier transform of the wave vector dependent charge polarizability as

$$
\chi(\mathbf{R})=\int \frac{d^{2} \mathbf{q}}{(2 \pi)^{2}} e^{i \mathbf{q} \mathbf{R}} \Pi(\mathbf{q})
$$

where $\Pi(\mathbf{q})$ is given by

$$
\Pi(q)=\frac{g_{s} g_{v}}{L^{2}} \sum_{\mathbf{k}, s^{\prime}} \frac{f_{s k}-f_{s^{\prime} \mathbf{k}^{\prime}}}{\epsilon_{s \mathbf{k}}-\epsilon_{s^{\prime} \mathbf{k}^{\prime}}} F_{s s^{\prime}}\left(\mathbf{k}, \mathbf{k}^{\prime}\right),
$$

where $g_{s}\left(g_{v}\right)$ is the spin (valley) degeneracy factor, $s, s^{\prime}= \pm 1$ represent band indices for electron and holes, $\mathbf{k}^{\prime}=\mathbf{k}+\mathbf{q}, \mathrm{F}_{s s^{\prime}}\left(k, k^{\prime}\right)=\left|\left\langle\psi_{k s} \mid \psi_{k^{\prime} s^{\prime}}\right\rangle\right|^{2}$ and $f_{s k}=$ $1 /\left(1+\exp \left(\epsilon_{s k}-\mu\right) / k_{B} T\right)$ is the Fermi distribution function. The Fermi distribution function at zero temperature is given by $f_{k s}=\Theta\left(\epsilon_{F}-\epsilon_{k s}\right)$ where $\epsilon_{F}$ is the Fermi function and $\Theta$ the step function.

\subsubsection{Charge polarizability in monolayer graphene}

Intrinsic case: First we consider the intrinsic case in monolayer graphene in which Fermi energy $E_{f}$ is located at zero. Basically it happens when graphene is not biased or gated. At zero temperature it implies that all the states in the valence band are filled $\left(f_{-k}=1\right)$ while all the states in the conduction band are empty $\left(f_{+k}=0\right)$. As we discussed before, monolayer graphene has a linear dispersion $\epsilon_{s}(k)=s \gamma|\mathbf{k}|$ with eigenfunction $\psi_{s k}=\left(e^{-i \theta_{k}}, s\right) / \sqrt{2}$. Thus the overlap function for monolayer graphene is given by $F_{s s^{\prime}}\left(k, k^{\prime}\right)=\left(1+s s^{\prime} \cos \theta\right) / 2$. By performing summations over $s$ and $s^{\prime}$ in Eq. 108, the polarizability function for monolayer graphene can be written as

$$
\Pi_{M L G}^{\text {int }}(q)=\frac{g_{c} g_{v}}{2} \int \frac{d^{2} \mathbf{k}}{(2 \pi)^{2}}\left(\frac{1-\cos \theta}{\epsilon_{+k}-\epsilon_{-k^{\prime}}}-\frac{1-\cos \theta}{\epsilon_{-k}+\epsilon_{+k^{\prime}}}\right),
$$


where $\cos \theta=(|\mathbf{k}|+|\mathbf{q}| \cos \phi) /|\mathbf{k}+\mathbf{q}|$ and $\theta$ is the angle between $\mathbf{k}$ and $\mathbf{k}+\mathbf{q}$. Solving the integral in Eq. 109 leads to

$$
\Pi_{M L G}^{i n t}(q)=\frac{g_{s} g_{v} k_{F}}{2 \pi \gamma} q
$$

Thus the intrinsic charge polarizability function in MLG is linear in q. The quantity $\mathrm{N}_{0}^{M L G}=\frac{g_{s} g_{v} k_{F}}{2 \pi \gamma}$ is the density state of monolayer graphene.

Extrinsic case: For the extrinsic case (doped or gated graphene), at zero temperature all the states below the Fermi energy are filled. Here we restrict ourselves to $n$-doped case where the Fermi energy is located in conduction band $f_{-k}=1$ and $f_{+k}=\Theta\left(k_{F}-|\mathbf{k}|\right)$. By defining $\tilde{f}_{s k}=f_{s k}-f_{s k}^{0}$ with $f_{s k}^{0}$ as the Fermi function for the intrinsic case, Eq. 108 can be written as

$$
\Pi^{\text {ext }}(q)_{M L G}=\frac{2 g_{s} g_{v}}{L^{2}} \sum_{k, s s^{\prime}} \tilde{f}_{s k} \frac{F_{s s^{\prime}}\left(k, k^{\prime}\right)}{\epsilon_{s k}-\epsilon_{s^{\prime} k^{\prime}}}
$$

where the overlap function is given by $F_{s s^{\prime}}\left(k, k^{\prime}\right)=\left(1+s s^{\prime} \cos \theta\right) / 2$. Performing summation over s' yields

$$
\Pi_{M L G}^{e x t}(q)=\frac{2 g_{s} g_{v}}{L^{2}} \sum_{k, s} f^{+}\left(\epsilon_{k}\right) \frac{1}{2}\left(\frac{1+s \cos \theta}{2}+\frac{1-s \cos \theta}{2}\right),
$$

By some straight-forward calculations one can write

$$
\Pi_{M L G}^{e x t}(q)=\frac{2 g_{s} g_{v}}{\gamma} \int \frac{d^{2} \mathbf{k}}{(2 \pi)^{2}} f^{+}\left(\epsilon_{k}\right) \frac{2 k+q \cos (\phi)}{q^{2}+2 k q \cos (\phi)},
$$

where $f^{+}\left(\epsilon_{k}\right)=\sum_{s} \tilde{f}_{s k}$. Using relations

$$
\begin{aligned}
& \int_{0}^{2 \pi} \frac{d \theta}{X+\cos \theta}=\frac{2 \pi}{\sqrt{X^{2}-1}} \Theta(|X|-1), \\
& \int_{0}^{2 \pi} \frac{d \theta \cos \theta}{X+\cos \theta}=-2 \pi\left(\frac{X}{\sqrt{X^{2}-1}}-1\right) \Theta(|X|-1)+2 \pi \Theta(1-|X|), \\
& \int_{0}^{2 \pi} \frac{d \theta \cos ^{2} \theta}{X+\cos \theta}=2 \pi \frac{X^{2}}{\sqrt{X^{2}-1}} \Theta(|X|-1)-2 \pi X .
\end{aligned}
$$

for solving the angular part of integral in Eq. 113, the charge polarizability reads as

$$
\Pi_{M L G}^{e x t}(q)=\frac{2 g_{s} g_{v}}{2 \pi \gamma}\left(\int_{0}^{\infty} f^{+}\left(\epsilon_{k}\right) d k-\int_{0}^{q / 2} f^{+}\left(\epsilon_{k}\right) \sqrt{1-(2 k / q)^{2}} d k\right)
$$


At zero temperature $f^{+}(\epsilon)=\Theta\left(\epsilon_{F}-\epsilon\right)$, and thus extrinsic part of charge polarizability for monolayer graphene is obtained as (Ando, 2006; Hwang and Das Sarma, 2007)

$$
\Pi_{M L G}^{e x t}(q)=\left\{\begin{array}{l}
\frac{g_{s} g_{v} k_{F}}{2 \pi \gamma}\left(1-\frac{\pi}{4} \frac{q}{2 k_{F}}\right) \quad \text { if } q \leqslant 2 k_{F}, \\
\frac{g_{s} g_{v} k_{F}}{2 \pi \gamma}\left(1-\frac{1}{2} \sqrt{1-\left(\frac{2 k_{F}}{q}\right)^{2}}-\frac{1}{2}\left(\frac{q}{2 k_{F}}\right) \sin ^{-1} \frac{2 k_{F}}{q}\right) \quad \text { if } q>2 k_{F} .
\end{array}\right.
$$

\subsubsection{Charge polarizability in bilayer graphene}

Intrinsic case: For calculating the intrinsic contribution of the charge polarizability for bilayer graphene we start again from Eq. 108. As we mentioned before, bilayer graphene has a parabolic dispersion as $\epsilon_{s}(k)=s k^{2} / 2 M$ with eigenfunction $\psi_{s k}=\left(e^{-i \theta_{k}}, s\right) / \sqrt{2}$ where $\theta_{k}=\tan ^{-1}\left(k_{y} / k_{x}\right)$ and $M$ is the effective mass of charge carriers. The overlap function in Eq. 108 for bilayer graphene is given by $\mathrm{F}_{s s^{\prime}}\left(k, k^{\prime}\right)=\left(1+s s^{\prime} \cos (2 \theta)\right) / 2$. For the intrinsic case the Fermi energy is at neutrality point $\left(E_{F}=0\right)$ and at zero temperature we have $f_{-k}=1$ and $f_{+k}=0$. So one can write the intrinsic charge polarizability in BLG as (Hwang and Das Sarma, 2008)

$$
\Pi_{\mathrm{BLG}}^{0}(q)=\frac{g_{\mathrm{c}} g_{v}}{2} \int \frac{\mathrm{d}^{2} \mathrm{k}}{(2 \pi)^{2}}\left(\frac{1-\cos 2 \theta}{\epsilon_{+\mathrm{k}}-\epsilon_{-\mathrm{k}^{\prime}}}-\frac{1-\cos 2 \theta}{\epsilon_{-\mathrm{k}}+\epsilon_{+\mathrm{k}^{\prime}}}\right),
$$

Solving the integral leads to

$$
\Pi_{\mathrm{BLG}}^{0}(\mathrm{q})=\frac{\mathrm{g}_{\mathrm{c}} \mathrm{g}_{v} M}{2 \pi} \log (4) .
$$

One can see that, in contrast to the intrinsic charge polarizability function in MLG which is linear in q, for BLG this function is constant for all wave vectors. The charge polarizability function for two dimensional electron gas (2DEG) is only constant when $q \leqslant 2 k_{F}$ (Ando et al., 1982).

Extrinsic case: For extrinsic case as for monolayer graphene we consider ndoped bilayer graphene. In order to solve the problem analytically, one can separate the polarizability due to interband and intraband transitions and rewrite 
Eq. 108 as $\Pi(q)=\Pi^{i n t r a}(q)+\Pi^{i n t e r}(q)$. For bilayer graphene these functions are given by

$$
\begin{aligned}
& \Pi_{\mathrm{BLG}}^{\mathrm{intra}}(q)=\frac{-\mathrm{g}_{\mathrm{s}} \mathrm{g}_{v}}{\mathrm{~L}^{2}} \sum_{\mathbf{k}, \mathrm{s}} \frac{\mathrm{f}_{\mathrm{sk}}-\mathrm{f}_{\mathrm{s} \mathbf{k}^{\prime}}}{\epsilon_{\mathrm{sk}}-\epsilon_{\mathrm{s} \mathbf{k}^{\prime}}} \frac{1+\cos 2 \theta}{2}, \\
& \Pi_{\mathrm{BLG}}^{\mathrm{inter}}(q)=\frac{-\mathrm{g}_{\mathrm{s}} \mathrm{g}_{v}}{\mathrm{~L}^{2}} \sum_{\mathbf{k}, \mathrm{s}} \frac{\mathrm{f}_{\mathrm{sk}}-\mathrm{f}_{-\mathrm{s} \mathbf{k}^{\prime}}}{\epsilon_{\mathrm{sk}}-\epsilon_{-s \mathbf{k}^{\prime}}} \frac{1-\cos 2 \theta}{2} .
\end{aligned}
$$

After angular integration and then integration over k, Eq. 118 leads to intraband and interband polarizability function for BLG (Hwang and Das Sarma, 2008)

$$
\begin{aligned}
& \Pi_{\mathrm{BLG}}^{\text {intra }}(q)=\left\{\begin{array}{l}
\frac{g_{c} g_{v} M}{2 \pi}\left(1-\frac{q^{2}}{2 k_{F}^{2}}\right) \quad \text { if } q \leqslant k_{F}, \\
\frac{g_{c} g_{v} M}{2 \pi}\left(\frac{q^{2}}{2 k_{F}^{2}}-2 \log \frac{q}{k_{F}}\right) \quad \text { if } k_{F}<q<2 k_{F}, \\
\frac{g_{c} g_{v} M}{2 \pi}\left(\frac{q^{2}}{2 k_{F}^{2}}-2 \log \frac{q}{k_{F}}-f_{B L G}(q)\right) \quad \text { if } k_{F} \geqslant 2 k_{F} .
\end{array}\right. \\
& \prod_{B L G}^{i n t e r}(q)=\left\{\begin{array}{l}
\frac{g_{c} g_{v} M}{2 \pi}\left(-1+\frac{q^{2}}{2 k_{F}^{2}}+g_{B L G}(q)\right) \quad \text { if } q \leqslant k_{F}, \\
\frac{g_{c} g_{v} M}{2 \pi}\left(-\frac{q^{2}}{2 k_{F}^{2}}+2 \log q+g_{B L G}(q)\right) \quad \text { if } k_{F}<q .
\end{array}\right.
\end{aligned}
$$

where

$$
\begin{aligned}
& f_{B L G}(q)=\frac{2 k_{F}^{2}+q^{2}}{2 k_{F}^{2} q} \sqrt{q^{2}-4 k_{F}^{2}}+\log \frac{q-\sqrt{q^{2}-4 k_{F}^{2}}}{q+\sqrt{q^{2}-4 k_{F}^{2}}}, \\
& g_{B L G}(q)=\frac{1}{2 k_{F}^{2}} \sqrt{4 k_{F}^{4}+q^{4}}-\log \left(\frac{k_{F}^{2}+\sqrt{4 k_{F}^{4}+q^{4} / 4}}{2 k_{F}^{2}}\right) .
\end{aligned}
$$

For angular integration in Eq. 118, we have used relations in Eq. 114, and notice that limits for integration over $k$ for intraband transition it is $\left(0, k_{F}\right)$ and for interband transition is $\left(k_{F}, \infty\right)$. Finally the polarizability function for doped BLG is given by

$$
\Pi_{B L G}(q)=N_{B L G}^{0}\left(g_{B L G}(q)-f_{B L G}(q) \Theta\left(q-2 k_{F}\right)\right)
$$

where $N_{B L G}^{0}=\frac{g_{c} g_{v} M}{2 \pi}$ is the density of states for BLG. In Fig. 15 the static charge 


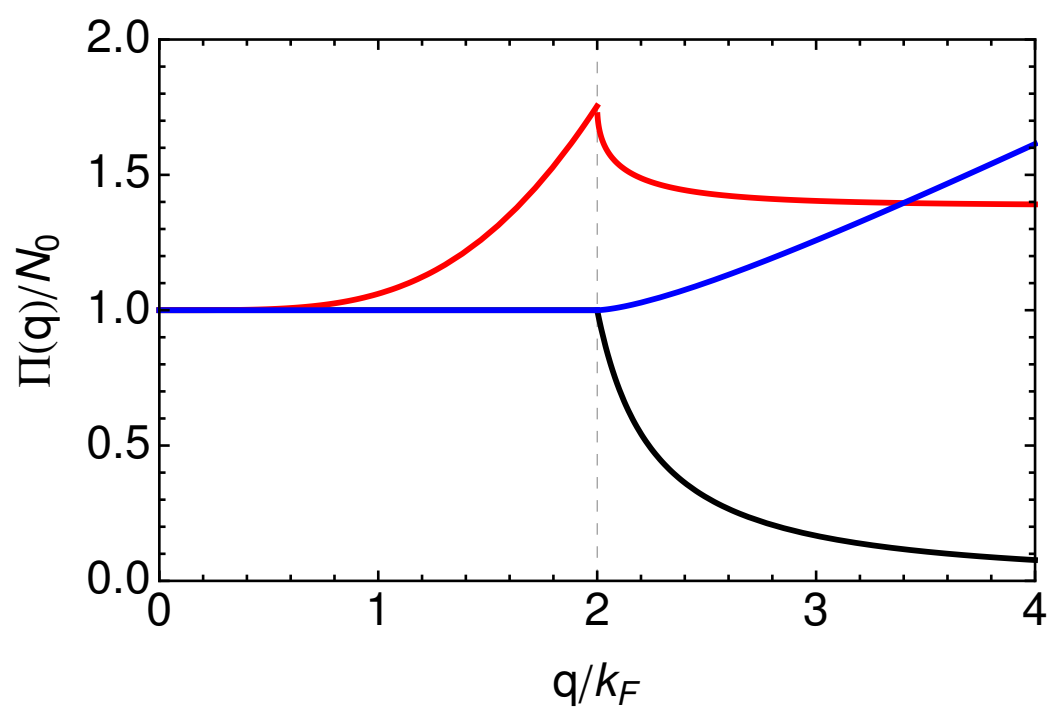

Figure 15: Static charge polarizability of monolayer graphene (blue) and bilayer graphene (red) and ${ }_{2}$ DEG (black). $\mathrm{N}_{0}$ denotes the density of states of the system.

polarizability for MLG, BLG and 2DEG is shown as a function of the wave vector $q$. In the following, we discuss three different situations for the charge polarizability of MLG and BLG (Hwang and Das Sarma, 2007, 2008; Ando, 2009; Ando et al., 1982).

a) $\mathbf{q}<2 \mathrm{k}_{\mathrm{F}}$ :

In this case, for MLG, the contribution form the interband and intraband polarizability can cancel each other out, and so $\Pi(q)$ up to $q=2 k_{F}$ is constant, which is similar to 2 DEG. However for BLG, intraband and interband parts of $\Pi(q)$ do not cancel each other specially when $q>k_{F}$, as shown in Fig. 15 it results in increasing the screening as $q$ increases. For $q=0$ the charge polarizability for MLG, BLG and 2DEG is equal to their density of states.

b) $\mathrm{q}=2 \mathrm{k}_{\mathrm{F}}$ :

In this case there is a qualitative difference between the charge polarizabilities of MLG and BLG. The BLG polarizability has a sharp cusp and its derivative is discontinuous at $q=2 k_{F}$ whereas in MLG the derivative of $\Pi(q)$ at $q=2 k_{F}$ is continuous. This is because at $q=2 k_{F}$ backscattering is not allowed in MLG and the overlap function $F_{s s^{\prime}}\left(k, k^{\prime}\right)$ vanishes at $q=2 k_{F}$, However in BLG at $q=2 k_{F}$ 
backscattering is enhanced resulting in singular behavior of $\Pi_{B L G}(q)$ at $q=2 k_{F}$. The derivative of the charge polarizability $\left(\frac{\mathrm{d} \Pi(\mathrm{q})}{\mathrm{dq}}\right)$ of ${ }_{2} \mathrm{DEG}$ is also not continuous at $q=2 k_{F}$ and shows a sharp cusp (Ando et al., 1982).

c) $\mathrm{q}>2 \mathrm{k}_{\mathrm{F}}$ :

For large momentum when $\mathrm{q}>2 \mathrm{k}_{\mathrm{F}}$, the BLG polarizability approaches its intrinsic value $\left(\Pi(q) \rightarrow \frac{g_{s} g_{v}}{2 \pi v_{F}} q\right)$, and this happens because interband transitions overcome the intraband transitions for a large wave vector q. For MLG the polarizability increases linearly with $\mathrm{q}$. The static polarizability for $2 \mathrm{DEG}$ falls off as $1 / \mathrm{q}^{2}$ for $\mathrm{q}>2 \mathrm{k}_{\mathrm{F}}$ (Ando et al., 1982).

\subsubsection{RKKY interaction}

The RKKY interaction (after Rudermann-Kittel-Kasuya-Yosida) is an indirect exchange interaction between two localized magnetic moments via the spin density of itinerant electrons in the host material (Ruderman and Kittel, 1954; Kasuya, 1956; Yosida, 1957). The direct interaction of a localized magnetic moment with its surrounding electrons induces an oscillation in the spin density of itinerant electrons, and this oscillation can then interact with another localized magnetic moment. The Hamiltonian describing direct interaction between localized magnetic moments $\mathbf{I}_{l}$ located at site $l$, and the localized spin density of electrons $\mathbf{S}_{l}$ is given by

$$
\mathrm{H}_{\text {int }}(\mathrm{r})=\mathrm{J} \sum_{\mathrm{l}} \mathrm{S}_{\mathrm{l} \cdot \mathrm{I}_{l},}
$$

where J is the exchange coupling constant. The RKKY Hamiltonian can be achieved from second-order perturbation expansion in J. By assuming that the magnetic moments are located at the interface of graphene and the substrate in order not to break any kind of symmetry, the RKKY Hamiltonian is proportional to the Fourier transform of the charge polarizability and is given by

$$
\mathrm{H}_{\mathrm{RKKY}}(\mathrm{r})=\mathrm{J}^{2} \mathrm{~S}_{1} \mathrm{~S}_{2} \Pi(\mathrm{r}),
$$


where $\Pi(r)$ is the Fourier transform of $\Pi(q)$ and $S_{1}$ and $S_{2}$ are localized spin moments.

In the case of intrinsic BLG, since $\Pi_{B L G}^{0}(q)=\frac{g_{s} g_{v} M}{2 \pi} \log 4$ is constant its Fourier transform simply becomes $\Pi_{\mathrm{BLG}}(\mathrm{r})=\frac{\mathrm{g}_{\mathrm{s}} \mathrm{g}_{v} \mathrm{M}}{2 \pi} \log 4 \delta(\mathrm{r})$ which shows that there is no magnetic order, and the long range RKKY interaction can not correlate magnetic moments. However for intrinsic MLG, $\Pi_{M L G}(r) \sim-1 / r^{3}$, which implies that moments are ferromagnetically coupled. The RKKY interaction for doped BLG is given by $\Pi_{B L G}(r) \sim \frac{\sin 2 k_{F} r}{\left(k_{F} r\right)^{2}}$ and thus its behavior is the same as for $2 D E G$, that is it decreases as $1 / r^{2}$. In contrast, $\Pi(r)$ for doped MLG has $1 / r^{3}$ behavior. It has been shown by Saremi (2007) that for bipartite lattices like graphene, if magnetic moments are located on the same sublattice, they are ferromagnetically coupled. However, they are anti-ferromagnetically coupled if the moments are on different sublattices.

\subsection{ELECTRICAL PROPERTIES OF $\mathrm{MoS}_{2}$}

Despite its huge potential for applications in electronic devices, there are two main reasons to consider alternatives to graphene. The first being that pristine graphene has no energy gap between conduction and valence bands, which effectively rules it out as a semiconductor. So for example, graphene based field effect transistors have a low on/off ratio and, attempts to open up a gap in graphene reduces its high mobility and adds complexity to the system. Secondly, due to the lightness of the carbon atoms, graphene has a very weak spin-orbit coupling (SOC). A strong intrinsic SOC would be, however, desirable for spintronic applications.

An important class of material that recently has attracted a lot of attention is transition-metal dichalcogenides (Cao et al., 2012; Zeng et al., 2012; Wang et al., 2012). Transition-metal dichalcogenides have been studied thoroughly for many years, however recent progresses in device fabrication technology and sample 

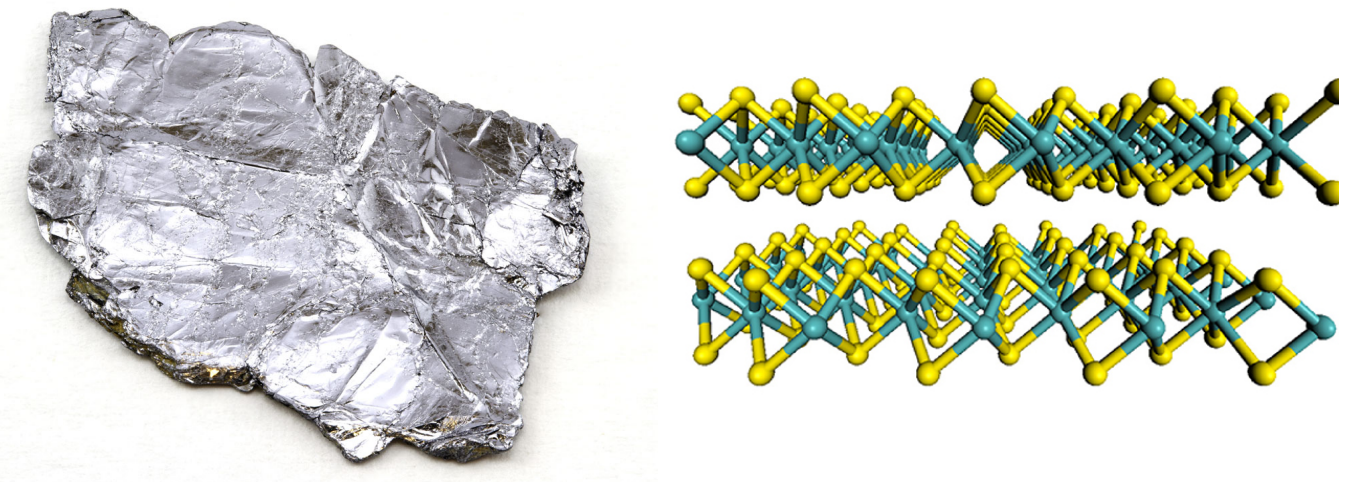

Figure 16: Bulk $\mathrm{MoS}_{2}$ crystal (left) (source: http:// genevalunch.com). $\mathrm{MoS}_{2}$ is a layered material and each layer is composed of a hexagonal lattice of sulfur atoms sandwiched between two hexagonal lattices of molybdenum atoms (right). The strong intralayer interactions between atoms are covalent whereas the weak interlayer interactions are of Van der Waals type.

preparation have triggered the investigation of two-dimensional layer thin films of this class of material. Transition-metal dichalcogenides have the general chemical formula of $\mathrm{MX}_{2}$ where $\mathrm{M}$ is a transition metal from group IV, V and VI and X is a chalcogen like sulfur. The most important compounds from this group of material are $\mathrm{MoS}_{2}, \mathrm{MoSe}_{2}, \mathrm{WS}_{2}$ and $\mathrm{WSe}_{2}$. In this class of material adjacent M-X-M layers are weakly stacked together to form the bulk crystal. So like graphite, transition-metal dichalcogenides are layered which implies that there is a strong in-plane covalent interaction between the atoms in each layer and a weak Van der Waals interaction between the atoms in different layers. Thus exploiting micromechanical cleavage is a suitable technique to split its bulk into individual atomic layers.

Monolayer $\mathrm{MoS}_{2}$ is one of the recent discoveries in material science with promising properties for electronic and spintronic applications. $\mathrm{MoS}_{2}$ belongs to the transition-metal dichalcogenides class of materials. Bulk $\mathrm{MoS}_{2}$ is a semiconductor with an indirect gap of $1.3 \mathrm{eV}$, which is a good candidate for photovoltaic and photocatalysts applications. However when it is cleaved to have only one 
layer it becomes a semiconductor with a direct band gap of $1.8 \mathrm{eV}$ across two inequivalent so called Dirac points $\mathbf{K}$ and $\mathbf{K}^{\prime}$ (see Fig. 17), which makes it a good candidate for optoelectronic applications.

The mobility of $\mathrm{MoS}_{2}$ has been measured between 0.5 and $3 \mathrm{~cm}^{2} / \mathrm{Vs}$ for different samples at room temperature which shows that high quality $2 \mathrm{D} \mathrm{MoS}_{2}$ layers produced by cleavage have almost the same carrier mobility as their bulk counterparts (Novoselov et al., 2005b).

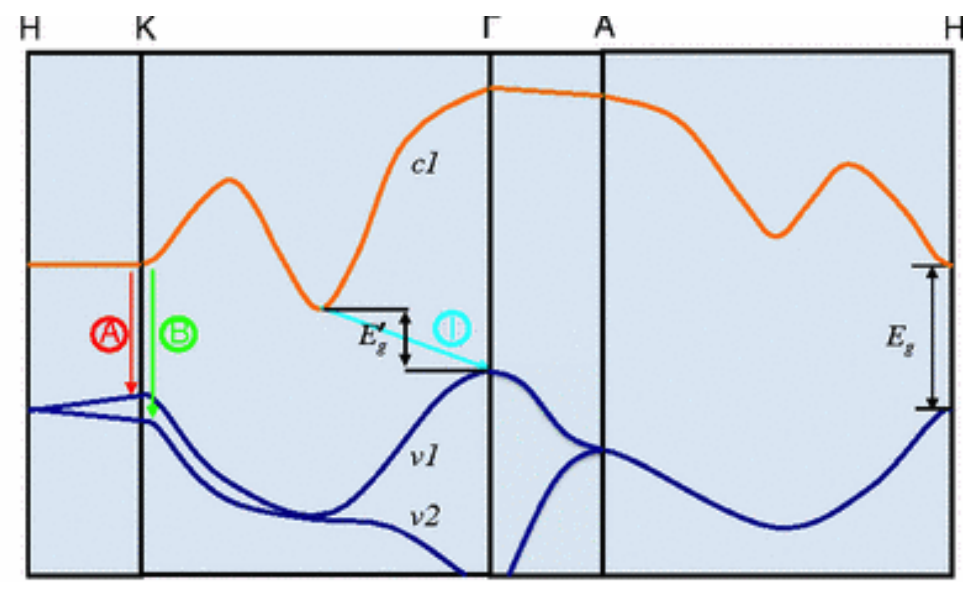

Figure 17: A simplified band structure of bulk $\mathrm{MoS}_{2}$ with the lowest conduction band $\mathrm{c1}$ and highest split valance bands v1 and v2. $\mathrm{E}_{g}^{\prime}$ indicates the indirect band gap of bulk $\mathrm{MoS}_{2}$ and $\mathrm{E}_{\mathrm{g}}$ shows the direct band gap of monolayer $\mathrm{MoS}_{2}$. Figure adopted from Mak et al. (2010).

Monolayer of $\mathrm{MoS}_{2}$ consist of two triangular lattice of $S$ atoms on the top of each other and one triangular lattice of Mo atoms sandwiched between the two layers of $\mathrm{S}$ atoms. Mo atoms are arranged to be in the middle of each triangular of the top and bottom layers (see Fig. 16). In Fig. 18 a top view of the lattice structure of monolayer $\mathrm{MoS}_{2}$ is shown. From this view, monolayer $\mathrm{MoS}_{2}$ lattice is like a honeycomb lattice of graphene with one major difference that different sublattices have different atoms. This arrangement gives rise to a broken inversion symmetry which in turn yields a relatively large band gap $(\sim 1.8 \mathrm{eV})$. In addition, monolayer $\mathrm{MoS}_{2}$ has strong SOC due to admixture of d orbitals of the heavy transition metal Mo, which renders it a good candidate for spintronic applications. 


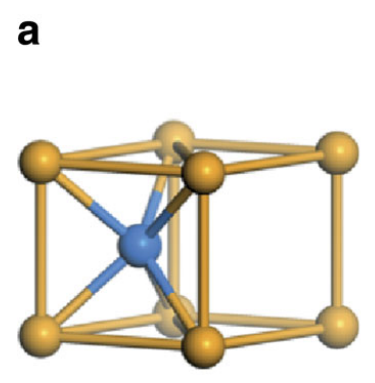

b

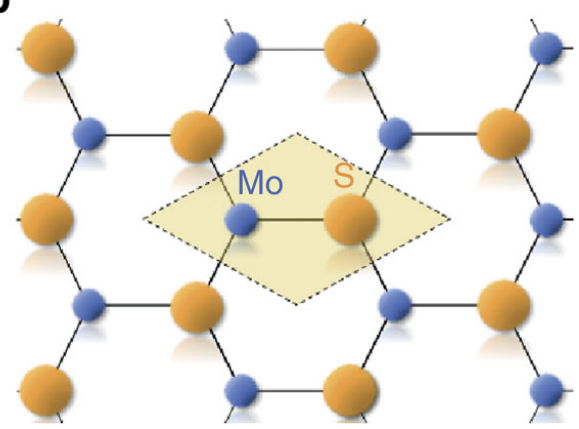

Figure 18: Lattice structure of monolayer $\mathrm{MoS}_{2}$ : a) A Mo atom (blue) in $\mathrm{MoS}_{2}$ lattice with coordination number 6 is surrounded with sulfur atoms. b) The top view of the $\mathrm{MoS}_{2}$ monolayer lattice. The unit cell of the lattice consist of two atoms shown by a shaded diamond. This view clearly shows the connection between the lattice structure of $\mathrm{MoS}_{2}$ and a honeycomb lattice. The Mo and $\mathrm{S}$ atoms are located on different sublattices. This image has been taken from Cao et al. (2012).

The low energy effective Hamiltonian of monolayer $\mathrm{MoS}_{2}$ has been suggested using the k.p perturbation theory (Xiao et al., 2012). To first order in $k$ the total Hamiltonian reads as

$$
\mathrm{H}=\operatorname{at}\left(\tau k_{x} \sigma_{x}+k_{y} \sigma_{y}\right)+\frac{\Delta}{2} \sigma_{z}-\lambda \tau \frac{\sigma_{z}-1}{2} s_{z}
$$

where $k_{\chi(y)}$ are components of wave vector $\mathbf{k}$ measured from valley $\mathbf{K}$ and $\mathbf{K}^{\prime}, \sigma$ and $s$ denote Pauli matrices acting on sublattice space and spin space respectively, $\mathrm{t} \simeq 1.1 \mathrm{eV}$ the hopping integral between the nearest neighbor atoms, $\mathrm{a} \simeq 3.193 \AA$ the lattice constant,$\tau= \pm$ the valley index and $\Delta=1.66 \mathrm{eV}$ is the direct band gap in $\mathrm{MoS}_{2}$. The last term in the Hamiltonian is due to SOC which has been approximated by the intra-atomic contribution L.S. The SOC splits the valance bands by $2 \lambda$ at $k=0$. This spin splitting is a consequence of inversion symmetry breaking and is opposite at different valleys due to TRS and is of importance to spintronics, valleytronics and optoelectronics applications. For example by using circularly polarized light to optically excite the monolayer of $\mathrm{MoS}_{2}$, the valley population can be controlled (Xiao et al., 2012). This process is generally called 


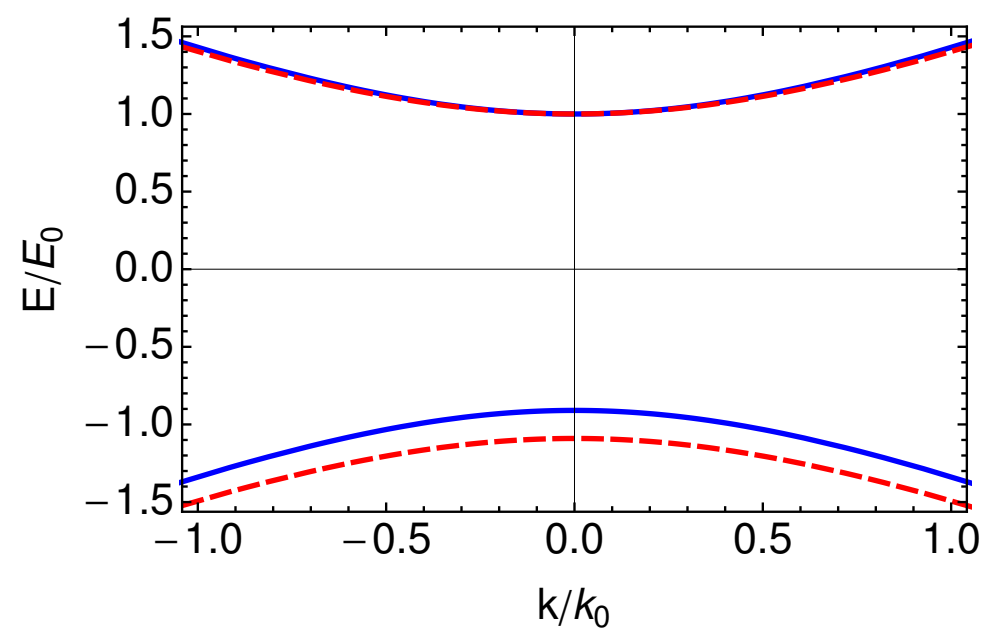

Figure 19: Spin-resolved band dispersions for monolayer $\mathrm{MoS}_{2}$ at the $\mathbf{K}$ point calculated from Eq. 124. Spin- $\uparrow(\downarrow)$ bands are shown as the blue solid (red dashed) curves. The unit scales for energy $E$ and wave vector $\mathbf{k}$ (measured from $\mathbf{K}$ ) are given in terms of band-structure parameters as $E_{0} \equiv \Delta / 2$ and $k_{0} \equiv \Delta /(2 a t)$. Reversal of all spin labels yields the corresponding band dispersions at the $\mathbf{K}^{\prime}(\equiv-\mathbf{K})$ point.

spin valley coupling and has been seen experimentally (Zeng et al., 2012; Cao et al., 2012). The dispersion relation corresponding to Hamiltonian Eq. 124 is shown in Fig. 19.

Recently there has been a generalization of the Hamiltonian Eq. (124) in which the quadratic terms in $k$ are responsible for giving electron and hole different masses (Rostami et al., 2013; Kormányos et al., 2013). This Hamiltonian is given by

$$
H=\operatorname{at}\left(\tau k_{x} \sigma_{x}+k_{y} \sigma_{y}\right)+\frac{\hbar^{2} k^{2}}{4 m}\left(\alpha+\beta \sigma_{z}\right)+\frac{\Delta}{2} \sigma_{z}-\lambda \tau \frac{\sigma_{z}-1}{2} s_{z}
$$

where $\alpha=0.43$ and $\beta=2.21$ are dimensionless parameters. In order to see the effect of the correction term (the term proportional to $k^{2}$ ), we plot the energy dispersion relation. Using the same Hamiltonian suggested by the authors of Rostami et al. (2013) and making it dimensionless by using the energy scale $\mathrm{E}_{0}=$ $\frac{\Delta}{2}$ we have:

$$
\bar{H}_{0}^{\tau} \equiv H_{0}^{\tau} / E_{0}=\bar{t}\left(\tau \bar{k}_{x} \hat{\sigma}_{x}+\bar{k}_{y} \hat{\sigma}_{y}\right)+\hat{\sigma}_{z}-\frac{\bar{\lambda} \tau}{2}\left(\hat{\sigma}_{z}-1\right) \otimes \hat{s}_{z}+\bar{k}^{2}\left(\bar{\alpha}+\bar{\beta} \hat{\sigma}_{z}\right),
$$



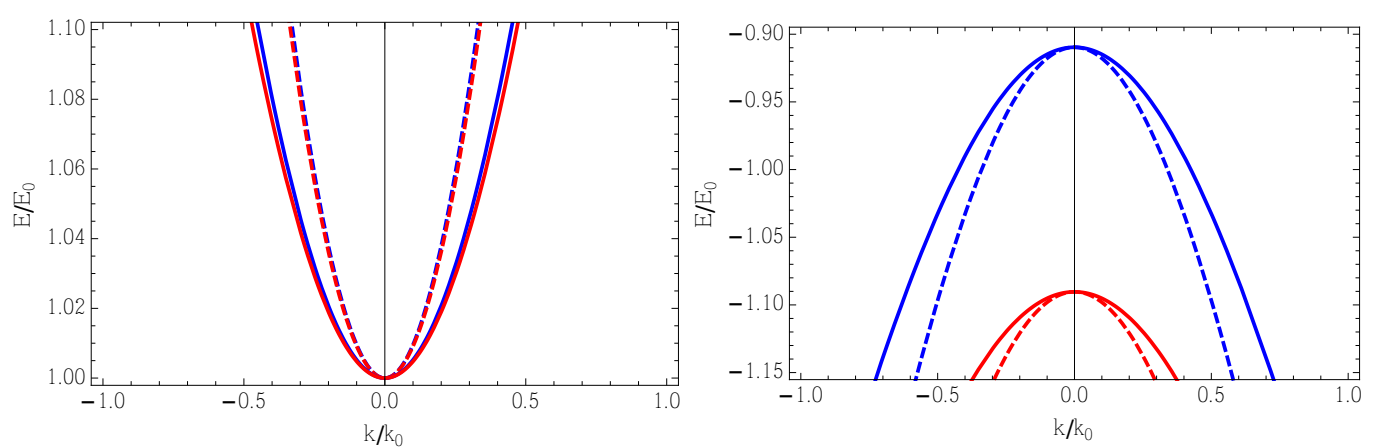

Figure 20: Calculated conduction (up) and valence (down) bands of monolayer $\mathrm{MoS}_{2}$. Blue and red curves correspond to real spin states $|\uparrow\rangle$ and $|\downarrow\rangle$ respectively. Effect of the correction term (the term proportional to $k^{2}$ ) is only included in the dashed curves. The energy scale here is $E_{0}=\Delta / 2$ and the structural parameters for $\mathrm{MoS}_{2}$ are $\alpha=0.43, \beta=2.21, \mathrm{t}_{0}=1.68 \mathrm{eV}, \Delta=1.9 \mathrm{eV}, \lambda=$ $0.08 \mathrm{eV}$.

where $\bar{k} \equiv \mathrm{ka}_{0}, \overline{\mathrm{t}} \equiv \mathrm{t} / \mathrm{E}_{0}$ and $\bar{\lambda} \equiv \lambda / \mathrm{E}_{0}$. We redefine dimensionless parameters as $\bar{\alpha} \equiv \frac{\hbar^{2}}{2 m_{0} a_{0}^{2} \Delta} \alpha$ and $\bar{\beta} \equiv \frac{\hbar^{2}}{2 m_{0} a_{0}^{2} \Delta} \beta$.

The eigenvalues of Hamiltonian (273) are given by

$$
E_{\mu s}(\bar{k})=\bar{k}^{2} \bar{\alpha}+\frac{\bar{\lambda} s \tau}{2}+\mu \frac{1}{2} \sqrt{4+4 s \bar{\lambda} \tau+\bar{\lambda}^{2}+4 \bar{k}^{2}\left(\bar{t}^{2}+\bar{\beta}\left(2+\bar{k}^{2} \bar{\beta}+s \bar{\lambda} \tau\right)\right)},
$$

where $\mu$ and $s$ are band indices for electron/hole branches and spin respectively. In Fig. 20 the dispersion relation of monolayer $\mathrm{MoS}_{2}$ with and without the correction term is shown. The dashed lines include the term proportional to $k^{2}$ whereas in solid curves this term is absent. 


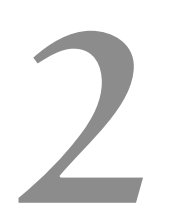

ELECTRONIC PROPERTIES OF BILAYER GRAPHENE RINGS

As we discussed in chapter 1 , electrons and holes in graphene come from two inequivalent valleys named $\mathrm{K}$ and $\mathrm{K}^{\prime}$, and as a result, graphene in the absence of inter-valley scattering processes is valley degenerate. Breaking this valley degeneracy opens the door to another intriguing application of graphene which is valleytronics (Xiao et al., 2007; Rycerz et al., 2007) or simply using valley degrees of freedom to transfer information. It has been shown that ring confinement and applying magnetic flux can provide a controllable way for lifting the valley degeneracy in monolayer graphene rings (MLGR) (Recher et al., 2007). In bilayer graphene a tunable mass gap can be opened up by applying a perpendicular electric field (McCann, 2006). Therefore, one can trap electron and hole carriers in a ring shape configuration using a bias voltage to have a bilayer graphene ring (BLGR). This kind of confinement is not possible in MLGR due to Klein tunneling.

Here in this chapter, we investigate the electronic properties of BLGRs using an analytical approach to calculate the electronic spectrum of a BLGR with finite width. We also calculate the persistent current (PC) induced by applying a magnetic flux for different number of non-interacting electrons in the ring. We then demonstrate how Rashba SOC, which can take place by breaking the inversion symmetry, affects energy eigenstates in BLGR. Finally we report our results on PC in BLGR in the presence of Rashba SOC.

\subsection{ENERGY LEVELS IN BLGR USING A TWO-BAND HAMILTONIAN}

In this section, we suggest an analytical solution for studying the electronic properties of a BLGR built by Bernal stacking of two MLGRs. For this purpose, we use 


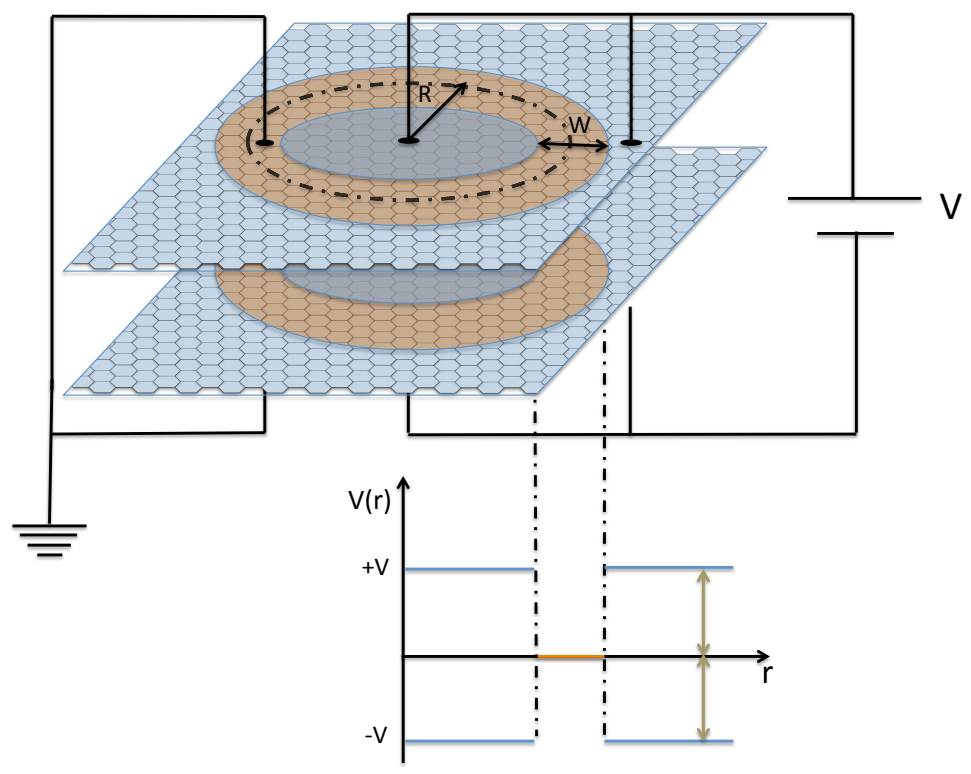

Figure 21: Energy profile of a bilayer graphene ring with radius $\mathrm{R}$ and width $\mathrm{W}$. The bias voltage $\mathrm{V}$ is responsible for confining electrons and holes in a ring shape.

the low energy effective Hamiltonian for bilayer graphene which was introduced by McCann and Falko (2006). The valley isotropic Hamiltonian of BLG is given by

$$
\mathcal{H}_{0}=\left(\begin{array}{cc}
0 & -\frac{\pi^{\dagger^{2}}}{2 M} \\
-\frac{\pi^{2}}{2 M} & 0
\end{array}\right)=\frac{-1}{2 M}\left(\pi^{\dagger^{2}} \sigma_{+}+\pi^{2} \sigma_{-}\right)
$$

in which, the two component basis for valley $\mathrm{K}$ is $\left(\phi_{A_{1}}, \phi_{\mathrm{B}_{2}}\right)$ and for valley $\mathrm{K}^{\prime}$ is $\left(\phi_{B_{2}}, \phi_{A_{1}}\right) . \phi_{A_{1}}$ and $\phi_{B_{2}}$ correspond to two different sublattices (denoted by A and B) from different layers (denoted by 1 and 2). Here, as we discussed in Chap. $1, \pi=p_{x}+i p_{y}, M$ is the effective mass and $\sigma_{-}=\sigma_{+}^{\dagger} \equiv \frac{1}{2}\left(\sigma_{x}-i \sigma_{y}\right)$ where $\sigma_{x, y, z}$ are Pauli matrices acting on the sublattice space. We define our ring by using an external bias $V(r)$ to confine the low energy electron and hole states in a ring shape region with width $\mathrm{W}$ and radius $\mathrm{R}$ as shown in Fig. 21. The bias potential appears in the Hamiltonian as the asymmetry between onsite energies in the two layers, so the valley isotropic form of the Hamiltonian for the BLGR (McCann and Falko, 2006) is

$$
\mathcal{H}^{\tau}=\mathcal{H}_{0}+\tau \mathrm{V}(\mathrm{r}) \sigma_{z}
$$


the term proportional to $\sigma_{z}$ is a mass term which confines the particles in the ring and $\tau=+1(-1)$ is the valley degree of freedom corresponding to valley $K\left(K^{\prime}\right)$ and $\mathrm{V}(\mathrm{r})$ is defined as

$$
\left\{\begin{array}{l}
V(r)=0 \text { for } R-\frac{W}{2}<r<R+\frac{W}{2}, \\
V(r)=V \text { otherwise. }
\end{array}\right.
$$

A perpendicular magnetic flux $\Phi$ threading the BLGR can be introduced by using the vector potential $\mathbf{A}=\left(\frac{\Phi}{2 \pi \mathrm{r}}\right) \hat{\boldsymbol{e}_{\boldsymbol{\phi}}}$ which alters momentum operator $\mathbf{p} \rightarrow \mathbf{p}+$ eA. For solving the eigenvalue equation $\mathcal{H}^{\tau} \psi=E^{\tau} \psi$ for BLGR, we write the Hamiltonian in polar coordinates using the following relations for $\pi$ and $\pi^{\dagger}$

$$
\begin{aligned}
& \pi=i \hbar\left(-\partial_{\mathrm{r}}+\frac{l_{z}+\frac{\Phi}{\Phi_{0}}-1}{r}\right) e^{i \phi} \\
& \pi^{\dagger}=-i \hbar\left(\partial_{\mathrm{r}}+\frac{l_{z}+\frac{\Phi}{\Phi_{0}}+1}{r}\right) e^{-i \phi},
\end{aligned}
$$

where $l_{z}=-i \partial_{\phi}$ is the angular momentum in the $z$ direction and $\Phi_{0}=\frac{2 \pi}{e}$. One can simply write the following relations as well

$$
\begin{aligned}
& \pi^{2}=-\hbar^{2}\left(-\partial_{r}+\frac{l_{z}+\frac{\Phi}{\Phi_{0}}-1}{r}\right)\left(-\partial_{r}+\frac{l_{z}+\frac{\Phi}{\Phi_{0}}-2}{r}\right) e^{2 i \phi} \\
& \pi^{\dagger^{2}}=-\hbar^{2}\left(\partial_{r}+\frac{l_{z}+\frac{\Phi}{\Phi_{0}}+1}{r}\right)\left(\partial_{r}+\frac{l_{z}+\frac{\Phi}{\Phi_{0}}+2}{r}\right) e^{-2 i \phi} .
\end{aligned}
$$

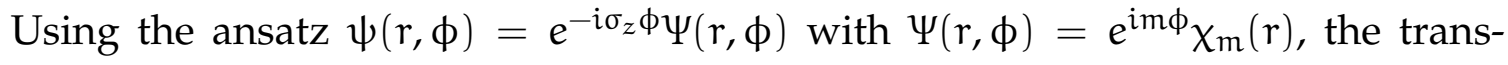
formed energy eigenvalue relation

$$
\tilde{\mathcal{H}} \Psi(\mathrm{r}, \phi)=\mathrm{E} \Psi(\mathrm{r}, \phi)
$$

and $\tilde{\mathcal{H}}=e^{i \sigma_{z} \phi} \mathcal{H} e^{-i \sigma_{z} \phi}$ can be written as

$$
\begin{aligned}
\tilde{\mathcal{H}}^{\tau} \chi_{\bar{m}}^{\tau}(r)= & \frac{\hbar^{2}}{2 M}\left[\left(\partial_{r}+\frac{\bar{m}}{r}\right)\left(\partial_{r}+\frac{\bar{m}+1}{r}\right) \sigma_{+}+\left(-\partial_{r}+\frac{\bar{m}}{r}\right)\left(-\partial_{r}+\frac{\bar{m}-1}{r}\right) \sigma_{-}\right. \\
& \left.+\tau V \sigma_{z}\right] \chi_{\bar{m}}^{\tau}(r)=E^{\tau} \chi_{\bar{m}}^{\tau}(r),
\end{aligned}
$$


where $\chi^{\tau}(r) \equiv\left(\begin{array}{c}\chi_{A}^{\tau} \\ \chi_{B}^{\tau}\end{array}\right)$ is a two component spinor with $\chi_{A}\left(\chi_{B}\right)$ the radial part of the envelope function for the probability amplitudes of the sublattices $A(B)$, and $\overline{\mathrm{m}} \equiv \mathrm{m}+\frac{\Phi}{\Phi_{0}}$ where $\mathrm{m}$ is the angular momentum eigenvalue and in a closed ring, it can only have integer values, i.e. $m=0, \pm 1, \pm 2, \ldots$ due to rotational symmetry. This relation leads to two differential equations which couple $\chi_{A}$ and $\chi_{B}$ as

$$
\left\{\begin{array}{l}
\frac{\hbar^{2}}{2 M}\left(\partial_{r}+\frac{\bar{m}}{r}\right)\left(\partial_{r}+\frac{\bar{m}+1}{r}\right) \chi_{B}=(E-\tau V) \chi_{A}, \\
\frac{\hbar^{2}}{2 M}\left(-\partial_{r}+\frac{\bar{m}}{r}\right)\left(-\partial_{r}+\frac{\bar{m}-1}{r}\right) \chi_{A}=(E+\tau V) \chi_{B}
\end{array}\right.
$$

Considering the case in which $\mathrm{V}>\mathrm{E}$, the four linearly independent solutions of Eqs. (135) are Kelvin's functions $\operatorname{ber}_{m}(k r), b i_{m}(k r), \operatorname{ker}_{m}(k r)$ and $k e i_{m}(k r)$. The relationships between Kelvin's functions and Bessel functions are given by

$$
\begin{aligned}
& \operatorname{ber}_{m}(z) \pm i \operatorname{bei}_{m}(z)=J_{m}\left(z e^{ \pm \frac{3}{4} \pi i}\right) \\
& \operatorname{ker}_{m}(z) \pm i k e i_{m}(z)=e^{-\frac{1}{2} m \pi i} K_{m}\left(z e^{ \pm \frac{\pi i}{4}}\right)
\end{aligned}
$$

where $z$ is real (Abramowitz and Stegun, 1964). The functions $\operatorname{ber}_{m}(k r)$ and bei $i_{m}(k r)$ are finite at the origin but they diverge when $r \rightarrow+\infty$ for any value of $m$ whereas $\operatorname{ker}_{m}(k r)$ and $k e i_{m}(k r)$ are well-defined at infinity but diverge at origin. The solutions for the middle part of the ring $\left(R-\frac{W}{2}<r<R+\frac{W}{2}\right)$ where $\mathrm{V}=0$ are ordinary and modified Bessel functions which are $J_{m}(k r), Y_{m}(k r)$, $\mathrm{I}_{\mathrm{m}}(\mathrm{kr})$ and $\mathrm{K}_{\mathrm{m}}(\mathrm{kr})$. 
Knowing the solutions of Eq. 135, enables us to write the spinor part of the wave functions for the different regions of the ring with valley index $\tau$ as follows

$$
\begin{aligned}
& \chi_{1}=A_{1}\left(\begin{array}{c}
-\sqrt{\frac{\tau V+E}{\tau V-E}} b_{e i}(k r) \\
\operatorname{ber}_{m+1}(k r)
\end{array}\right)+A_{2}\left(\begin{array}{c}
\sqrt{\frac{\tau V+E}{\tau V-E}} \text { ber }_{m-1}(k r) \\
\operatorname{bei}_{m+1}(k r)
\end{array}\right), \\
& \chi_{2}=A_{3}\left(\begin{array}{c}
\operatorname{sgn}(E) J_{m-1}\left(k^{\prime} r\right) \\
J_{m+1}\left(k^{\prime} r\right)
\end{array}\right)+A_{4}\left(\begin{array}{c}
\operatorname{sgn}(E) Y_{m-1}\left(k^{\prime} r\right) \\
Y_{m+1}\left(k^{\prime} r\right)
\end{array}\right) \\
& +A_{5}\left(\begin{array}{c}
\operatorname{sgn}(E) I_{m-1}\left(k^{\prime} r\right) \\
I_{m+1}\left(k^{\prime} r\right)
\end{array}\right)+A_{6}\left(\begin{array}{c}
\operatorname{sgn}(E) K_{m-1}\left(k^{\prime} r\right) \\
K_{m+1}\left(k^{\prime} r\right)
\end{array}\right), \\
& \chi_{3}=A_{7}\left(\begin{array}{c}
-\sqrt{\frac{\tau V+E}{\tau V-E}} k e i_{m-1}(k r) \\
k e r_{m+1}(k r)
\end{array}\right)+A_{8}\left(\begin{array}{c}
\sqrt{\frac{\tau V+E}{\tau V-E}} k e r_{m-1}(k r) \\
k e i_{m+1}(k r)
\end{array}\right) \text {, }
\end{aligned}
$$

where $\chi_{1}$ and $\chi_{3}$ are the radial part of the wave functions of the inner part $r<$ $R-\frac{W}{2}$ and outer part $r>R+\frac{W}{2}$ of the ring respectively and $\chi_{2}$ is the radial wave function for the ring $R-\frac{W}{2}<r<R+\frac{W}{2}$ and wavevectors are defined as $k^{\prime}=\sqrt{\frac{2 M|E|}{\hbar^{2}}}$ and $k=\sqrt{\frac{2 M}{\hbar^{2}}}\left(V^{2}-E^{2}\right)^{\frac{1}{4}}$ and $\operatorname{sgn}(x)$ is the sign function. The wave function of the transformed Hamiltonin (Eq. 133) can then be written as

$$
\Psi(r, \phi)=e^{i m \phi}\left(\begin{array}{l}
\chi_{A}^{\tau}(r) \\
\chi_{B}^{\tau}(r)
\end{array}\right) .
$$

In order to find the energy levels of BLGR, we should apply proper boundary conditions for the continuity of the wave functions

$$
\left\{\begin{array}{l}
\left.\Psi_{1}\right|_{\mathrm{r}=\mathrm{R}-\frac{\mathrm{W}}{2}}=\left.\Psi_{2}\right|_{\mathrm{r}=\mathrm{R}-\frac{\mathrm{W}}{2}}, \\
\left.\Psi_{2}\right|_{\mathrm{r}=\mathrm{R}+\frac{\mathrm{W}}{2}}=\left.\Psi_{3}\right|_{\mathrm{r}=\mathrm{R}+\frac{\mathrm{W}}{2}},
\end{array}\right.
$$

and the probability flux

$$
\left\{\begin{array}{l}
\left.\tilde{v}_{\mathrm{r}} \Psi_{1}\right|_{\mathrm{r}=\mathrm{R}-\frac{\mathrm{W}}{2}}=\left.\tilde{v}_{\mathrm{r}} \Psi_{2}\right|_{\mathrm{r}=\mathrm{R}-\frac{\mathrm{W}}{2}}, \\
\left.\tilde{v}_{\mathrm{r}} \Psi_{2}\right|_{\mathrm{r}=\mathrm{R}+\frac{\mathrm{W}}{2}}=\left.\tilde{v}_{\mathrm{r}} \Psi_{3}\right|_{\mathrm{r}=\mathrm{R}+\frac{\mathrm{W}}{2}},
\end{array}\right.
$$



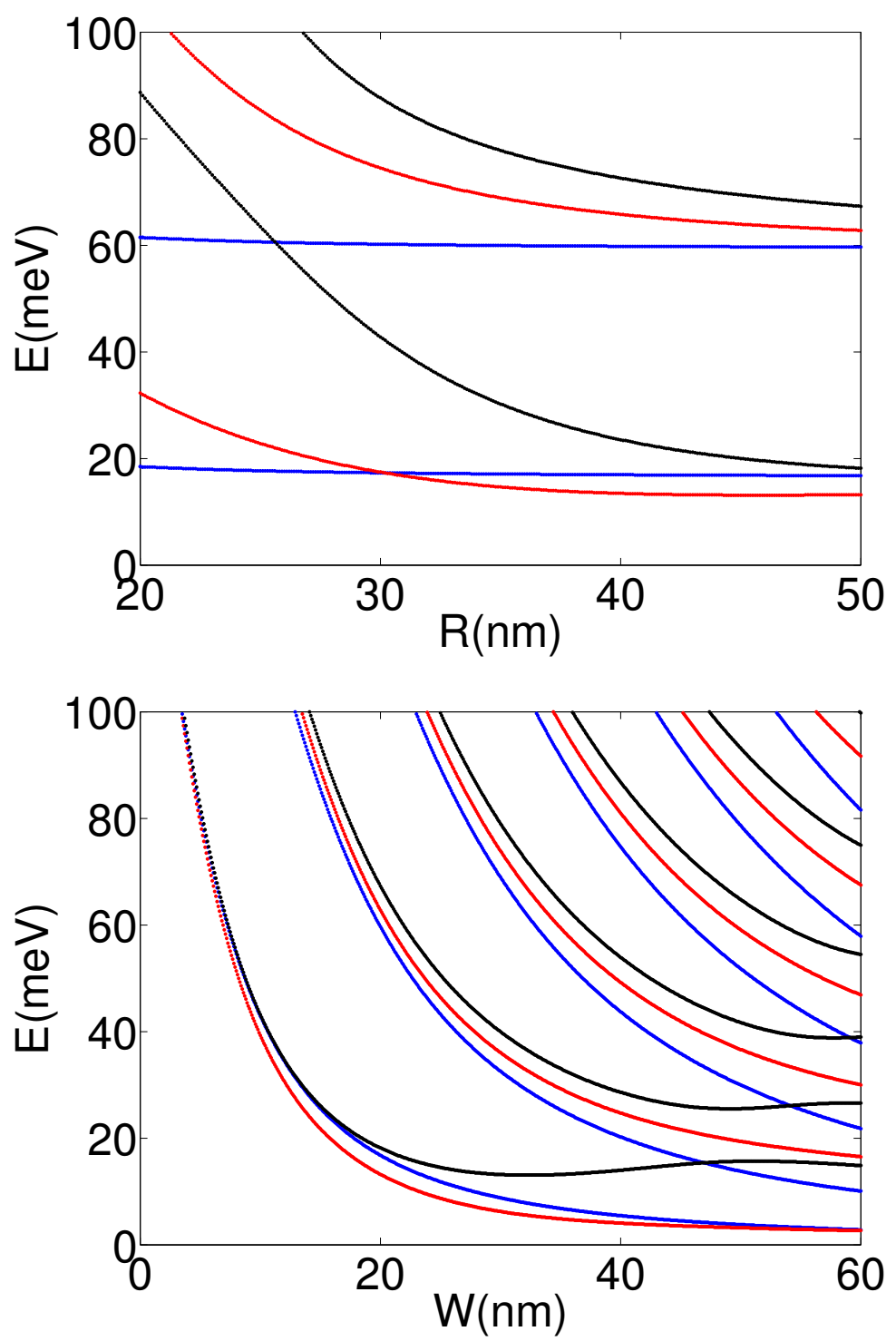

Figure 22: Energy levels of a BLGR with bias potential $\mathrm{V}=150 \mathrm{meV}$ as a function of radius (R) of the ring (upper panel) with width $\mathrm{W}=20 \mathrm{~nm}$ and as a function of width $(\mathrm{W})$ of the ring (lower panel) with radius $\mathrm{R}=50 \mathrm{~nm}$. The blue, red and black curves correspond to $\mathrm{m}=0,5,-5$ respectively. 
at interfaces $r=R-\frac{W}{2}$ and $r=R+\frac{W}{2}$, where $\tilde{v}_{r}$ is the transformed velocity operator (see 2.1.1).

These conditions lead to a system of equations from which the energy eigenstates can be found. The energy levels of a BLGR with bias voltage $V=150 \mathrm{~nm}$ as a function of width $(\mathrm{W})$ and $\operatorname{radius}(\mathrm{R})$ of the ring are shown in Fig. 22. The BLGR energy levels with negative energy (not shown in the plot) are obtained by symmetry $E^{\tau}(m)=-E^{\tau}(-m)$ and this symmetry can be understood from the energy eigenvalue relation Eq. 135. It comes from the fact that in each valley $\tau$, in the absence of inter-valley scattering, effective time-reversal symmetry (TRS) $(\mathbf{p} \rightarrow-\mathbf{p}, \boldsymbol{\sigma} \rightarrow-\boldsymbol{\sigma}, \tau \rightarrow \tau)$ is broken. However, in the absence of an applied magnetic field $(\Phi=0)$, we expect to have $E^{\tau}(m)=E^{-\tau}(-m)$ between states in different valleys since the real TRS is present.

The results from Fig. 22 show that the number of energy levels in the ring increases by increasing the width of the ring. They also show that for $m \neq 0$, there is an electron-hole asymmetry in the system. There is a very weak dependence on the radius of the ring for $m=0$, however this changes considerably for larger values of $|\mathrm{m}|$ especially when the ring radius is smaller than $40 \mathrm{~nm}$.

Recently Zarenia et al. (2009) have studied electronic properties of a BLGR with finite and zero width (Zarenia et al., 2010a,b) using a finite element method to solve the energy eigenvalue relation of the four-band Hamiltonian. In the case of a finite width ring they have seen anti-crossing between energy levels in BLGR in the presence and in the absence of an applied magnetic flux (see Fig. 23). The reason for having such anti-crossings is not clear. However our results are qualitatively in good agreement with the results reported by Zarenia et al. (2009) (see Fig. 23).

In Fig. 24 energy levels in a ring with bias voltage $V=150 \mathrm{meV}$ are plotted as a function of angular momentum eigenvalue $m$ for different width and radius of the ring. It shows that the lowest level has a non-symmetric saddle point which separates the two local minima which is different from the case of monolayer graphene (Zarenia et al., 2010a,b). In the lower panel of Fig. 24 the energy levels for different radius $R=40,50,60 \mathrm{~nm}$ are plotted and which shows that for small 

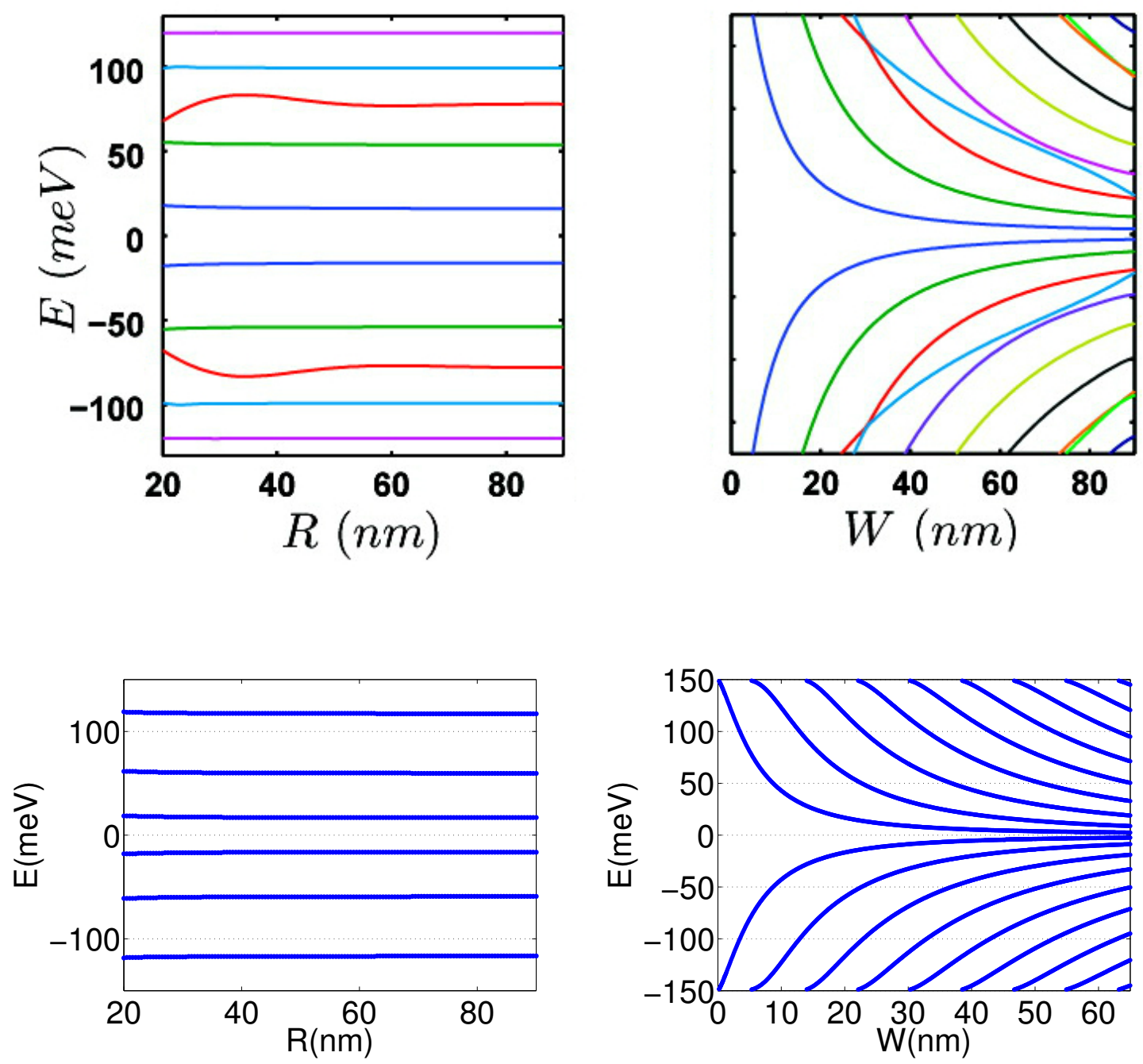

Figure 23: Lowest energy levels of a BLGR with bias potential $V=150 \mathrm{meV}$ as a function of ring radius $R$ (left panels) and as a function of width $W$ (right panels). Top panels are copied from Zarenia et al. (2009). In the right panels the width of the ring is $\mathrm{W}=20 \mathrm{~nm}$ and in the left panels the ring radius is $\mathrm{R}=50 \mathrm{~nm}$. Here angular momentum eigenvalue is zero $(m=0)$. 
values of $m$ the energy levels are not very sensitive to the change of the ring radius. However we can see that for larger values of $|\mathrm{m}|$ the energy levels are influenced by the changing in the ring radius. In the upper panel of Fig. 24 the energy levels for different width are plotted which shows that the number of energy levels in the ring increases, by increasing the width and also the minima in the lowest energy subband, occur at smaller values of $|\mathrm{m}|$.

\subsubsection{Calculating the velocity operator}

In order to find the radial component of the velocity operator, we start with the relations

$$
\begin{aligned}
& \hat{v}_{x}=\frac{i}{\hbar}[\mathcal{H}, x]=\frac{-1}{M}\left(\pi_{+} \sigma_{+}+\pi_{-} \sigma_{-}\right), \\
& \hat{v}_{y}=\frac{i}{\hbar}[\mathcal{H}, y]=\frac{i}{M}\left(\pi_{+} \sigma_{+}-\pi_{-} \sigma_{-}\right),
\end{aligned}
$$

where $\mathcal{H}$ is the Hamiltonian of BLGR Eq. 129 and $\hat{v}_{x}$ and $\hat{v}_{y}$ are the velocity operators in $x$ and $y$ directions. It is straightforward to write the radial component of the velocity $\hat{v}_{\mathrm{r}}=\hat{v}_{\mathrm{x}} \cos (\phi)+\hat{v}_{\mathrm{y}} \sin (\phi)$ as

$$
\hat{v}_{\mathrm{r}}=\frac{-1}{M}\left(e^{-i \phi} \pi_{+} \sigma_{+}+e^{i \phi} \pi_{-} \sigma_{-}\right)
$$

Using the polar form of operators $\pi_{ \pm}=\mp i \hbar e^{\mp i \phi}\left( \pm \partial_{r}+\frac{l_{z}}{r}\right)$ results in

$$
\hat{v}_{r}=\frac{-1}{M} \times\left[-i \hbar\left(\partial_{r}+\frac{l_{z}+2}{r}\right) e^{-2 i \phi} \sigma_{+}+i \hbar\left(-\partial_{r}+\frac{l_{z}-2}{r}\right) e^{2 i \phi} \sigma_{-}\right] .
$$

Since we have transformed Hamiltonian using the operator $U=e^{i \sigma_{z} \phi}$, we should also transform the velocity operator in Eq. 143 using the same ansatz that we applied to the Hamiltonian of the system, that is

$$
\tilde{v}_{\mathrm{r}}=U \hat{v}_{\mathrm{r}} \mathrm{U}^{\dagger}=e^{i \sigma_{z} \phi} \hat{v}_{\mathrm{r}} e^{-i \sigma_{z} \phi}
$$



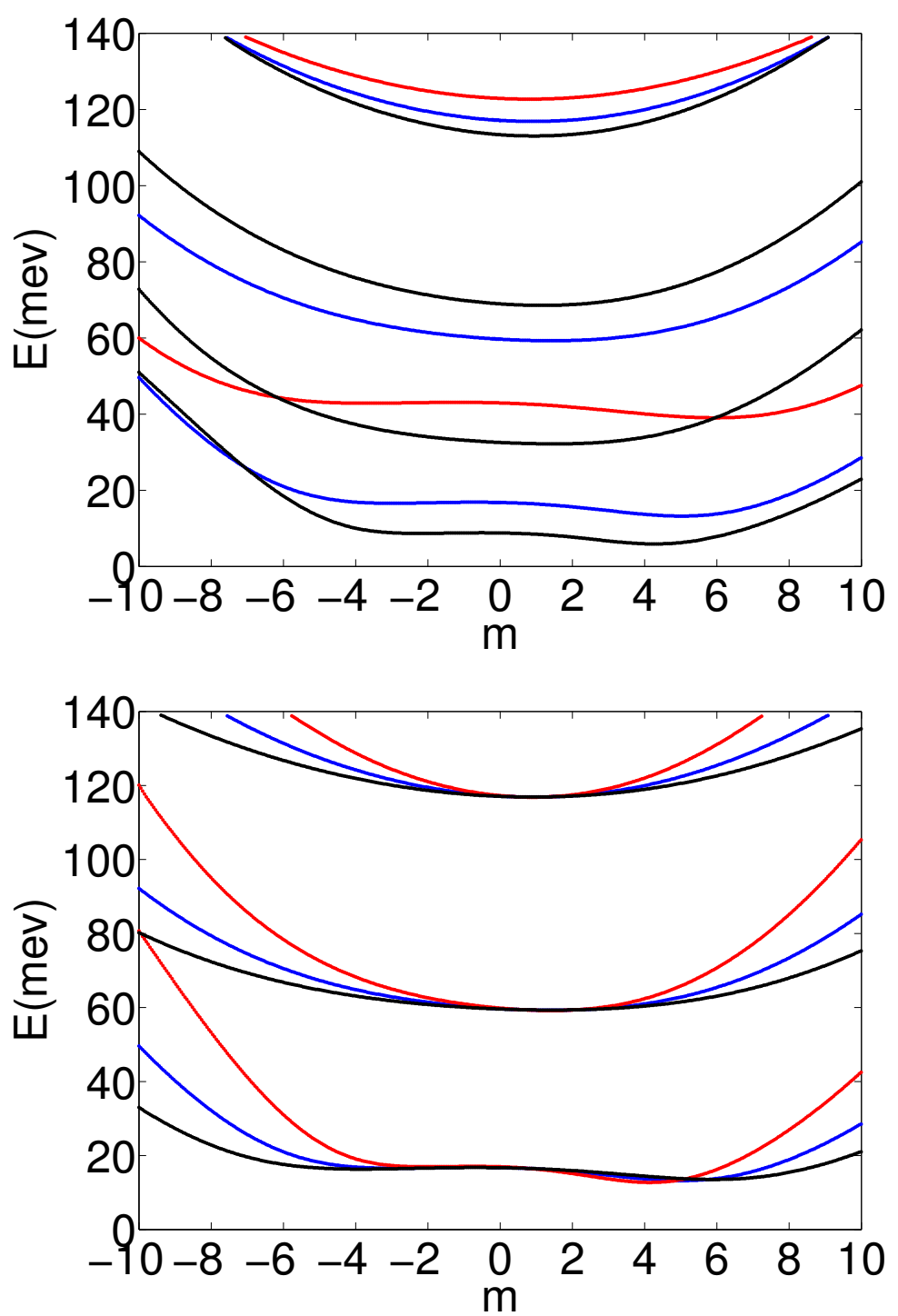

Figure 24: Energy levels of a BLGR with bias potential $V=150 \mathrm{meV}$ versus angular momentum $\mathrm{m}$. In the upper panel the radius of the ring is $\mathrm{R}=50 \mathrm{~nm}$ and width of the ring is $W=10 \mathrm{~nm}$ (red), $\mathrm{W}=20 \mathrm{~nm}$ (blue) and $\mathrm{W}=30 \mathrm{~nm}$ (black). In the lower panel the width of the ring is $\mathrm{W}=2 \mathrm{O} \mathrm{nm}$ and radius of the ring is $\mathrm{R}=4 \mathrm{O}$ $\mathrm{nm}$ (red), $\mathrm{R}=5 \mathrm{O} \mathrm{nm}$ (blue) and $\mathrm{R}=6 \mathrm{o} \mathrm{nm}$ (black). 
and using the following relations

$$
e^{i \sigma_{z} \phi} \sigma_{ \pm} e^{-i \sigma_{z} \phi}=\sigma_{ \pm} e^{ \pm 2 i \phi}
$$

the radial component of velocity operator corresponds to transformed Hamiltonian $\tilde{\mathcal{H}}$ read as

$$
\tilde{v}_{\mathrm{r}}=\frac{-1}{M} \times\left[-i \hbar\left(\partial_{\mathrm{r}}+\frac{l_{z}-\sigma_{z}+2}{r}\right) \sigma_{+}+i \hbar\left(-\partial_{\mathrm{r}}+\frac{l_{z}+\sigma_{z}+2}{r}\right) \sigma_{-}\right] .
$$

\subsection{COMPARISON BETWEEN TWO-BAND AND FOUR-BAND HAMILTONIAN IN BLGR}

So far in our calculations we have used the two-band low energy effective Hamiltonian (McCann and Falko, 2006). Towards better understanding the validity of two-band Hamiltonian when the bias voltage is smaller than the interlayer hopping $(\mathrm{V}<\mathrm{t})$, and also comparing our results with previous works (Zarenia et al., 2009, 2010a,b), we obtain the energy eigenstates of a BLGR using four-band Hamiltonian. This Hamiltonian for BLG with basis $\left(\chi_{A_{1}}^{*}, \chi_{B_{1}}^{*}, \chi_{A_{2}}^{*}, \chi_{B_{2}}^{*}\right)$ is given by (McCann and Falko, 2006)

$$
\mathcal{H}=\left(\begin{array}{cccc}
\mathrm{V} & v_{\mathrm{f}} \pi & 0 & \mathrm{t} \\
v_{\mathrm{f}} \pi^{\dagger} & \mathrm{V} & 0 & 0 \\
0 & 0 & -\mathrm{V} & v_{\mathrm{f}} \pi \\
\mathrm{t} & 0 & v_{\mathrm{f}} \pi^{\dagger} & -\mathrm{V}
\end{array}\right)
$$

where $A_{i}$ and $B_{i}$ denote different sublattices in the ith layer and $t \simeq 400 \mathrm{meV}$ is the interlayer hopping amplitude between the $A$ and $B$ sublattices on top of each other from different layers. $v_{\mathrm{f}} \simeq 10^{6} \mathrm{~m} / \mathrm{s}$ is the Fermi velocity of electrons in monolayer graphene and $\mathrm{V}$ is the bias potential between the layers and $\pi=$ $p_{x}+i p_{y}$. For using the same approach as for the two-band Hamiltonian, we write the four-band Hamiltonian in a compact form as

$$
\mathcal{H}=v_{\mathrm{f}} \mathbb{1} \otimes\left(\pi^{\dagger} \sigma_{-}+\pi \sigma_{+}\right)+\mathrm{t}\left(\lambda_{+} \sigma_{+}+\lambda_{-} \sigma_{-}\right)+V \sigma_{z} \otimes \mathbb{1}
$$


where $\sigma$ and $\lambda$ are the Pauli matrices acting on sublattice and layer degree of freedom respectively and $\mathbb{1}$ is a $2 \times 2$ unit matrix. $\lambda_{+}, \lambda_{-}$and $\lambda_{z}$ are defined as

$$
\begin{aligned}
& \lambda_{+}=\left(\begin{array}{ll}
0 & 1 \\
0 & 0
\end{array}\right), \\
& \lambda_{-}=\left(\begin{array}{ll}
0 & 0 \\
1 & 0
\end{array}\right), \\
& \lambda_{z}=\left(\begin{array}{cc}
1 & 0 \\
0 & -1
\end{array}\right) .
\end{aligned}
$$

For solving the eigenvalue problem $\mathcal{H} \psi=E \psi$, we can use the ansatz $\psi(r, \phi)=$ $e^{-i \sigma_{z} \phi / 2} e^{i \lambda_{z} \phi / 2} \Psi(r, \phi)$ to obtain the transformed energy eigenvalue relation $\tilde{\mathcal{H}} \Psi(\mathrm{r}, \phi)=$ $E \Psi(r, \phi)$ where $\tilde{\mathcal{H}}=e^{i \sigma_{z} \phi / 2} e^{-i \lambda_{z} \phi / 2} \mathcal{H} e^{-i \sigma_{z} \phi / 2} e^{i \lambda_{z} \phi / 2}$. By using the polar representation for $\pi^{ \pm}$and the following relations

$$
\begin{aligned}
& e^{i \sigma_{z} \phi / 2} \sigma_{ \pm} e^{-i \sigma_{z} \phi / 2}=\sigma_{ \pm} e^{ \pm i \phi}, \\
& e^{-i \lambda_{z} \phi / 2} \lambda_{ \pm} e^{i \lambda_{z} \phi / 2}=\lambda_{ \pm} e^{\mp i \phi} \\
& e^{i \sigma_{z} \phi / 2} e^{-i \lambda_{z} \phi / 2} \pi_{ \pm} e^{-i \sigma_{z} \phi / 2} e^{i \lambda_{z} \phi / 2}=\mp i \hbar\left( \pm \partial_{r}+\frac{l_{z}+\frac{\Phi}{\Phi_{0}}-\frac{\sigma_{z}}{2}+\frac{\lambda_{z}}{2} \pm 1}{r}\right) e^{\mp i \phi},
\end{aligned}
$$

we can write the energy eigenvalue relation as

$$
\begin{aligned}
\tilde{\mathcal{H}} \Psi(\mathrm{r}, \phi)= & \left(i \hbar v _ { \mathrm { f } } \left[\left(-\partial_{\mathrm{r}}+\frac{l_{z}+\frac{\Phi}{\Phi_{0}}-\frac{\sigma_{z}}{2}+\frac{\lambda_{z}}{2}-1}{\mathrm{r}}\right) \lambda_{+}\right.\right. \\
& \left.\left.-\left(\partial_{\mathrm{r}}+\frac{\mathrm{l}_{z}+\frac{\Phi}{\Phi_{0}}-\frac{\sigma_{z}}{2}+\frac{\lambda_{z}}{2}+1}{\mathrm{r}}\right) \lambda_{-}\right]+\mathrm{t}\left(\lambda_{+} \sigma_{+}+\lambda_{-} \sigma_{-}\right)+V \sigma_{z}\right) \Psi(\mathrm{r}, \phi) .
\end{aligned}
$$

Now as before we use the ansatz $\Psi(r, \phi)=e^{i m \phi} \chi_{m}(r)$ to separate the radial and angular part of the solution, where $\chi_{m}(r)$ is a four component spinor. Using this ansatz we can write the energy eigenvalue relation as 

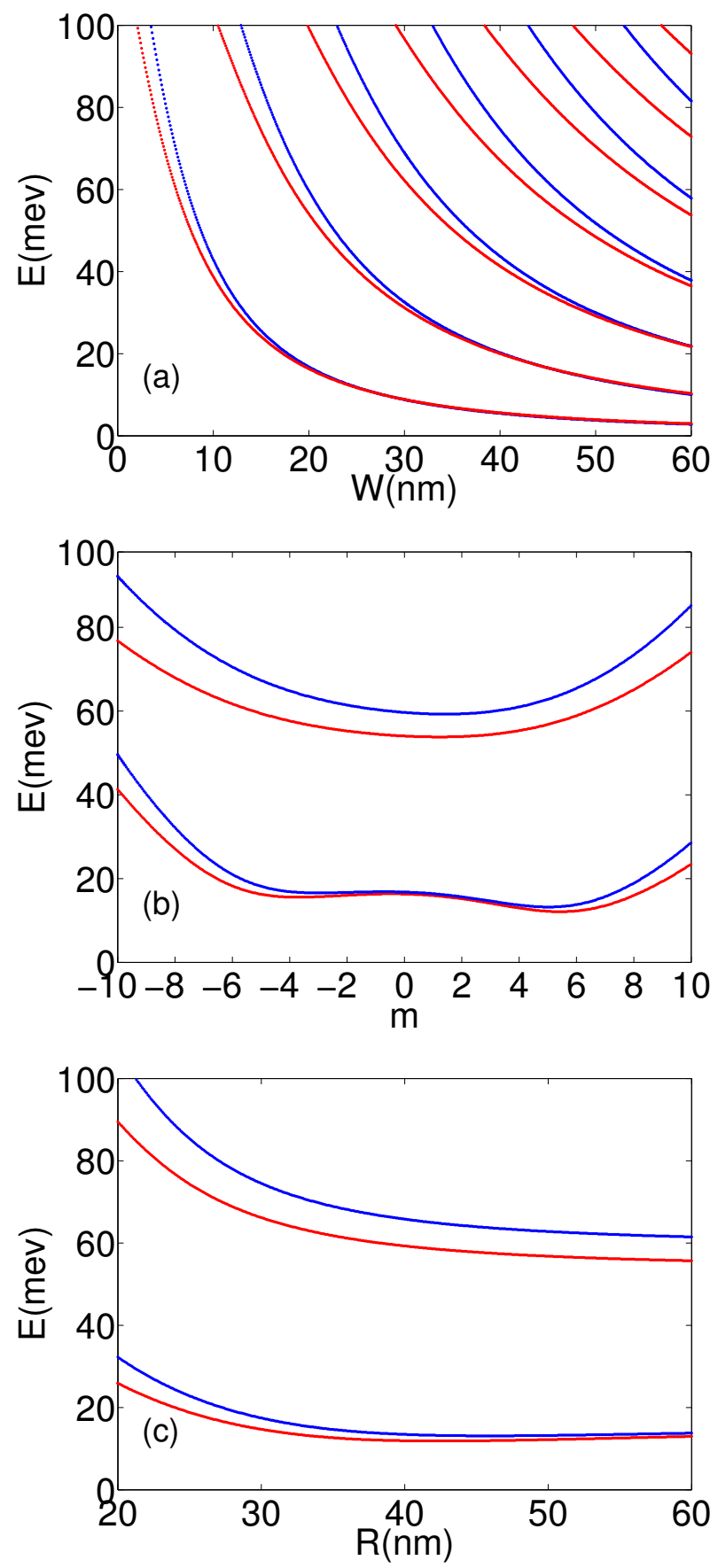

Figure 25: Comparison between the energy levels in a BLGR with $V=150 \mathrm{~nm}$ obtained from the two-band (blue) and four-band (red) Hamiltonian. The energy levels are plotted as function of width (W) in panel (a) with $\mathrm{m}=5$ and $\mathrm{R}=50 \mathrm{~nm}$, as a function of $\mathrm{m}$ in panel (b) where $\mathrm{W}=20 \mathrm{~nm}$ and $\mathrm{R}=50 \mathrm{~nm}$ and versus radius $(R)$ in panel (c) with $m=5$ and $W=20 \mathrm{~nm}$. The interlayer hopping for four-band Hamiltonian is $t \simeq 400 \mathrm{meV}$. 


$$
\left\{\begin{array}{l}
i\left(-\partial_{\mathrm{r}}+\frac{\overline{\mathrm{m}}-1}{\mathrm{r}}\right) \chi_{\mathrm{B}_{1}}+\mathrm{t} \chi_{\mathrm{B}_{2}}=(E-\tau \mathrm{V}) \chi_{\mathrm{A}_{1},} \\
-i\left(\partial_{\mathrm{r}}+\frac{\overline{\mathrm{m}}}{\mathrm{r}}\right) \chi_{\mathrm{A}_{1}}=(E-\tau V) \chi_{\mathrm{B}_{1}}, \\
i\left(-\partial_{\mathrm{r}}+\frac{\overline{\mathrm{m}}}{\mathrm{r}}\right) \chi_{\mathrm{B}_{2}}=(E+\tau V) \chi_{\mathrm{A}_{2}}, \\
-i\left(\partial_{\mathrm{r}}+\frac{\overline{\mathrm{m}}+1}{\mathrm{r}}\right) \chi_{\mathrm{A}_{2}}+\mathrm{t} \chi_{\mathrm{A}_{1}}=(E+\tau V) \chi_{\mathrm{B}_{2}},
\end{array}\right.
$$

where we have defined $\bar{m}=m+\frac{\Phi}{\Phi_{0}}$. So the radial part of the wave function based on the behavior of the Bessel functions in each region is given by

$x_{1} \quad=a_{1}\left(\begin{array}{c}J_{m}\left(k_{1} r\right) \\ \frac{i k_{1}}{V-E} J_{m-1}\left(k_{1} r\right) \\ \frac{i k_{1}\left(k_{1}{ }^{2}-(V-E)^{2}\right)}{t\left(V^{2}-E^{2}\right)} J_{m+1}\left(k_{1} r\right) \\ \frac{k_{1}{ }^{2}-(V-E)^{2}}{t(V-E)} J_{m}\left(k_{1} r\right)\end{array}\right)+a_{2}\left(\begin{array}{c}J_{m}\left(k_{2} r\right) \\ \frac{i k_{2}}{V-E} J_{m-1}\left(k_{2} r\right) \\ \frac{i k_{2}\left(k_{2}{ }^{2}-(V-E)^{2}\right)}{t\left(V^{2}-E^{2}\right)} J_{m+1}\left(k_{2} r\right) \\ \frac{k_{2}{ }^{2}-(V-E)^{2}}{t(V-E)} J_{m}\left(k_{2} r\right)\end{array}\right), r<R-\frac{W}{2}$

$X_{2} \quad=a_{3}\left(\begin{array}{c}J_{m}(k r) \\ \frac{i k}{V-E} J_{m-1}(k r) \\ \frac{i k\left(k^{2}-(V-E)^{2}\right)}{t\left(V^{2}-E^{2}\right)} J_{m+1}(k r) \\ \frac{k^{2}-(V-E)^{2}}{t(V-E)} J_{m}(k r)\end{array}\right)+a_{4}\left(\begin{array}{c}Y_{m}\left(k_{1} r\right) \\ \frac{i k}{V-E} Y_{m-1}\left(k_{1} r\right) \\ \frac{i k\left(k^{2}-(V-E)^{2}\right)}{t\left(V^{2}-E^{2}\right)} Y_{m+1}\left(k_{1} r\right) \\ \frac{k^{2}-(V-E)^{2}}{t(V-E)} Y_{m}\left(k_{1} r\right)\end{array}\right)$

$+a_{5}\left(\begin{array}{c}I_{m}\left(k^{\prime} r\right) \\ \frac{i k^{\prime}}{V-E} I_{m-1}\left(k^{\prime} r\right) \\ \frac{i k^{\prime}\left(k^{\prime 2}+(V-E)^{2}\right)}{t\left(V^{2}-E^{2}\right)} I_{m+1}\left(k^{\prime} r\right) \\ \frac{k^{\prime 2}+(V-E)^{2}}{-t(V-E)} I_{m}\left(k^{\prime} r\right)\end{array}\right)+a_{6}\left(\begin{array}{c}K_{m}\left(k^{\prime} r\right) \\ \frac{-i k^{\prime}}{V-E} K_{m-1}\left(k^{\prime} r\right) \\ \frac{-i k^{\prime}\left(k^{\prime 2}+(V-E)^{2}\right)}{t\left(V^{2}-E^{2}\right)} K_{m+1}\left(k^{\prime} r\right) \\ \frac{k^{\prime 2}+(V-E)^{2}}{-t(V-E)} K_{m}\left(k^{\prime} r\right)\end{array}\right), \quad R-\frac{W}{2}<r<R+\frac{W}{2}$

$x_{3}=a_{7}\left(\begin{array}{c}K_{m}\left(i k_{1} r\right) \\ \frac{k_{1}}{V-E} K_{m-1}\left(i k_{1} r\right) \\ \frac{k_{1}\left(-k_{1}^{2}+(V-E)^{2}\right)}{t\left(V^{2}-E^{2}\right)} K_{m+1}\left(i k_{1} r\right) \\ \frac{-k_{1}^{2}+(V-E)^{2}}{-t(V-E)} K_{m}\left(i k_{1} r\right)\end{array}\right)+a_{8}\left(\begin{array}{c}K_{m}\left(i k_{2} r\right) \\ \frac{k_{2}}{V-E} K_{m-1}\left(i k_{2} r\right) \\ \frac{k_{2}\left(-k_{2}^{2}+(V-E)^{2}\right)}{t\left(V^{2}-E^{2}\right)} K_{m+1}\left(i k_{2} r\right) \\ \frac{-k_{2}^{2}+(V-E)^{2}}{-t(V-E)} K_{m}\left(i k_{2} r\right)\end{array}\right), r>R+\frac{W}{2}$ 
where the wave vectors are defined as

$$
\begin{aligned}
k_{1} & =\sqrt{E^{2}+V^{2}-\sqrt{E^{2} t^{2}+4 E^{2} V^{2}-t^{2} V^{2}}} \\
k_{2} & =-\sqrt{E^{2}+V^{2}+\sqrt{E^{2} t^{2}+4 E^{2} V^{2}-t^{2} V^{2}}} \\
k & =\sqrt{E^{2}+\sqrt{E^{2} t^{2}}} \\
k^{\prime} & =\sqrt{-E^{2}+\sqrt{E^{2} t^{2}}} .
\end{aligned}
$$

Knowing the radial part of the wave function in different regions we can find the energy spectrum of the ring by applying proper boundary conditions, which in case of the four band Hamiltonian for BLGR is only matching the wave functions $(\Psi(r, \phi))$ at interfaces $r=R-\frac{W}{2}$ and $r=R+\frac{W}{2}$. In Fig. 25 the energy levels versus width $(\mathrm{W})$, angular momentum $(\mathrm{m})$ and radius of the ring $(\mathrm{R})$ are plotted using four-band (red) and two-band (blue) Hamiltonians. The parameters in our calculations are $t=400 \mathrm{meV}$ and $V=150 \mathrm{meV}$. It is clear that qualitatively the energy levels are the same and as one expects the two-band low energy effective Hamiltonian is consistent with the four-band model for lower energies where $\mathrm{E}<<\mathrm{V}<\mathrm{t}$.

\subsection{BLGR IN THE PRESENCE OF A MAGNETIC FIELD}

Broken valley degeneracy can be better seen by studying the persistent current (PC) induced in a BLGR when a perpendicular magnetic flux is applied. PC is a quantum effect related to the Aharonov-Bohm (Aharonov-Bohm (AB)) phase. The wave function of a charged particle traveling through a magnetic field picks up a phase called Aharonov-Bohm phase (Aharonov and Bohm, 1959; Büttiker et al., 1983; Washburn and Webb, 1986). At zero temperature $(T=0)$ and for non-interacting electrons the PC is given by (Recher et al., 2007)

$$
J=-\sum_{\tau} \sum_{m} \frac{d E_{m}^{\tau}}{d \Phi}
$$




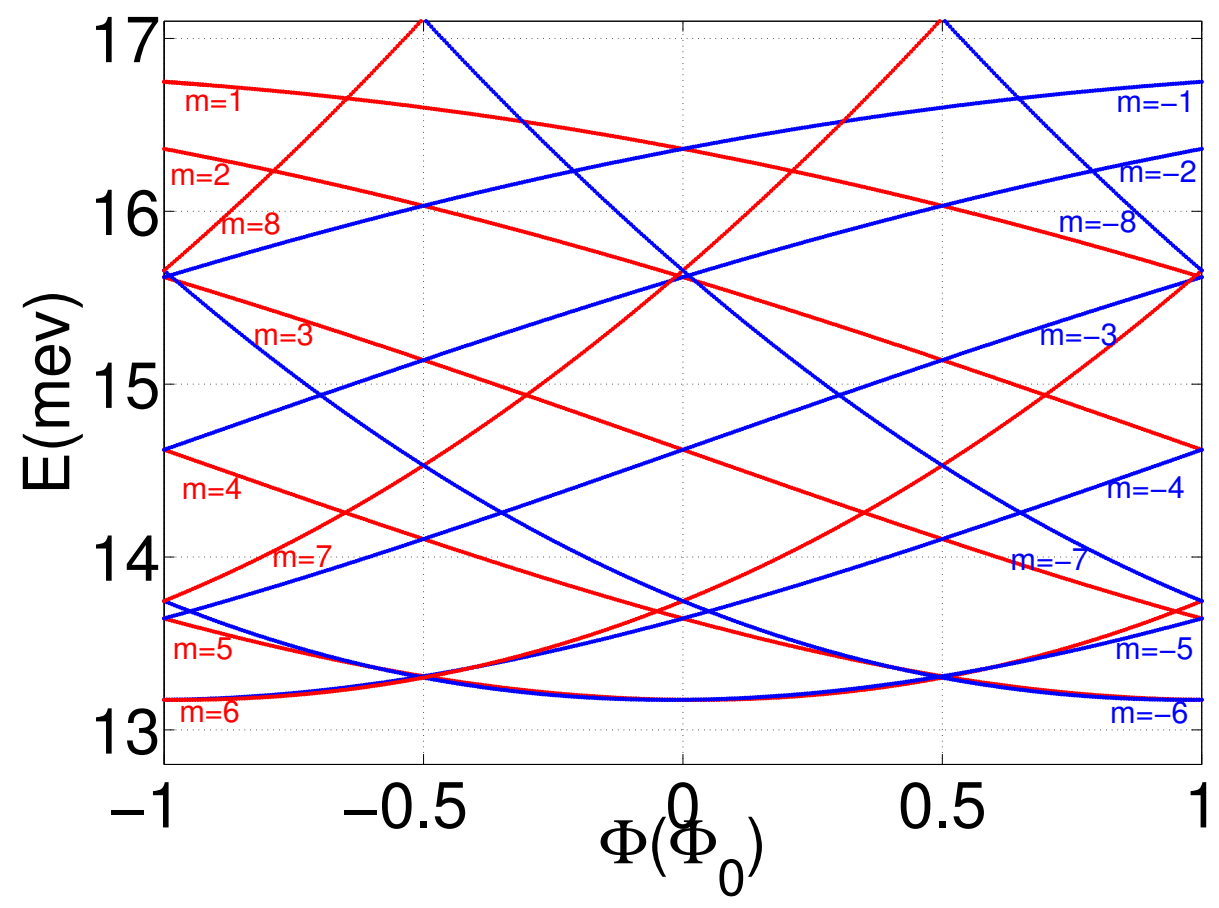

Figure 26: Energy levels in a BLGR with $V=150 \mathrm{meV}$, radius $R=50 \mathrm{~nm}$ and width $\mathrm{W}=20 \mathrm{~nm}$ versus magnetic field for different angular momentums $\mathrm{m}$. The red and the blue lines correspond to valley with indices $\tau=1$ and $\tau=-1$ respectively. (The value of $m$ for each level is written below the corresponding level with the same color.) 
where the sums are over all occupied states. Note that since we are calculating the PC in a closed ring, $m$ can only have integer values due to rotational symmetry. In Fig. 26 the lowest energy eigenstates are plotted as a function of $\Phi$ for different $m$ and valley indices $\tau$. As it is shown, the energy eigenstates with quantum numbers $\pm m$ from different valleys are degenerate in the absence of an applied magnetic flux $(\Phi=0)$. However this valley degeneracy is lifted by applying a finite flux $\Phi \neq 0$. As one expects the spectrum is periodic with periodicity $\Phi_{0}$. The eigenenergies from different valleys are related by $E_{m}^{\tau}(\Phi)=E_{-m}^{-\tau}(-\Phi)$ which can also be seen from Eq. 135 as it is preserved by replacing $\tau \rightarrow-\tau$, $\overline{\mathrm{m}} \rightarrow-\overline{\mathrm{m}}$.

The PC corresponding to the spectrum in Fig. 26 is shown in Fig. 27 for different number of particles $(\mathrm{N})$ in the ring considering the spin of the electrons. As one expects the PC has the periodicity of the energy spectrum and it is antisymmetric about $\Phi=0$. We note that the PC related to only one valley, e.g. $\tau=1$, is not zero when there is no flux applied to the system which shows the valley polarization. However, by considering the case where both valleys are populated with the same number of electrons, the PC including both valleys becomes zero when $\Phi=0$, whereas it shows finite structure with kinks at different values of magnetic flux $\Phi$. The non-zero PC implies the broken valley degeneracy due to applying magnetic flux. The kinks in the PC structure happen when the energy eigenvalues with different quantum numbers $\tau$ and $m$ intersect each other and causes discontinuity of the slope of the energy levels (energy curves which are periodic in $\Phi$ ) occupied by $\mathrm{N}$ electrons. Flux dependence of PC is sensitive to the number of electrons in the ring, for example kinks can disappear for even filling numbers larger than two when energy levels from a valley with angular momentum difference one $\left(\left|m^{\prime}-m\right|=1\right)$ intersect each other. That is the reason why in Fig. 27 the PC for $N_{K}=4$ does not have the kink structure located at $\Phi= \pm 0.5 \Phi_{0}$ as for $\mathrm{N}_{\mathrm{K}}=3$. 

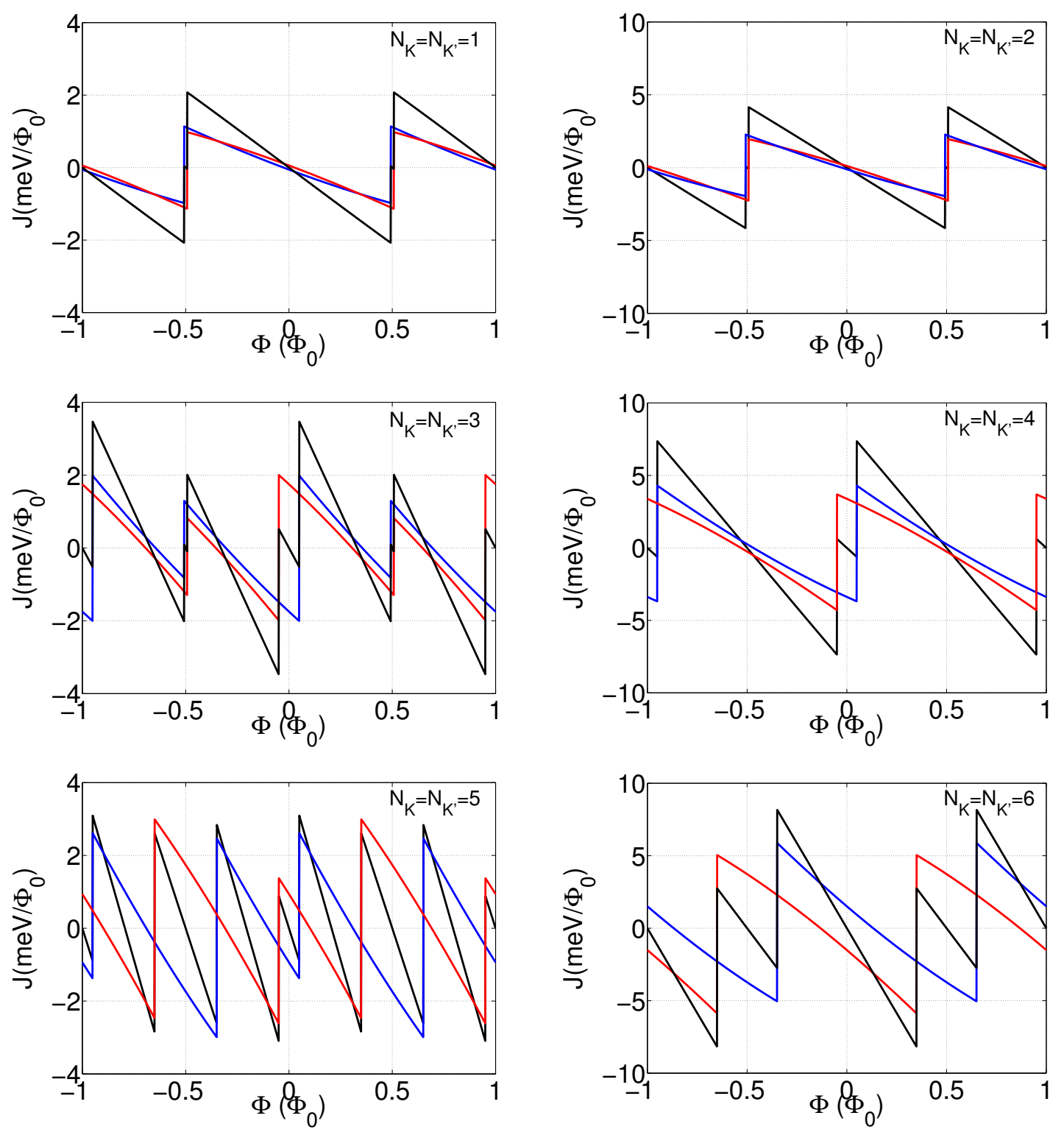

Figure 27: Persistent current as a function of magnetic field when the two valleys are equally occupied. $N_{K}$ and $N_{K^{\prime}}$ show the number of electrons in valley $K$ and $K^{\prime}$. The persistent current due to valley $K(\tau=1)$ is shown by red lines and for valley $K^{\prime}(\tau=-1)$ by blue lines. The persistent current considering both valleys is shown by black lines. 


\subsection{BLGR IN THE PRESENCE OF RASHBA SPIN ORBIT COUPLING}

A quantum ring with Rashba spin-orbit coupling interaction is very attractive for applications in spintronics (Nitta et al., 1999; Nitta, 2004). Rashba SOC in graphene can be generated if the lattice inversion symmetry is broken. In case of BLG, one way to break this symmetry is achieved by applying an external bias voltage (Qiao et al., 2013). The value of this coupling for a typical electric field is less than $1 \mathrm{meV}$. However recently a number of methods suggested to increase the Rashba SOC coupling such as placing graphene on different substrates or introducing impurities (Weeks et al., 2011). As an example, using Ni as the substrate to grow graphene epitaxially, can increase Rashba SOC up to $0.2 \mathrm{eV}$ (Varykhalov et al., 2008; Dedkov et al., 2008).

The effective low energy expression for Rashba SOC in BLG has been recently introduced (Mireles and Schliemann, 2012; Winkler and Zülicke, 2012) and is given by

$$
\mathcal{H}_{\mathrm{R}} \equiv \frac{\nu_{\mathrm{B}} i}{\hbar}\left(\pi \sigma_{-} \mathrm{s}_{+}-\pi^{\dagger} \sigma_{+} \mathrm{s}_{-}\right)
$$

where $v_{\mathrm{B}}$ shows the strength of the coupling which varies depending on the method used to break the lattice inversion symmetry, and $s_{ \pm}=s_{x} \pm i s_{y}$ in which $s_{x, y}$ are the Pauli matrices acting on real spin. So the Hamiltonian of a biased BLGR in the presence of Rashba SOC in valley $\mathrm{K}$ can be written as

$$
\mathcal{H}_{\mathrm{K}}=\frac{-1}{2 M}\left(\pi^{\dagger^{2}} \sigma_{+}+\pi^{2} \sigma_{-}\right)+\frac{\nu_{\mathrm{B}} i}{\hbar}\left(\pi \sigma_{-} s_{+}-\pi^{\dagger} \sigma_{+} s_{-}\right)+V \sigma_{z}
$$

For the valley $\mathrm{K}^{\prime}$ this Hamiltonian is given by $\mathcal{H}_{\mathrm{K}^{\prime}}=\Sigma_{y} \mathrm{H}_{\mathrm{K}} \Sigma_{y}^{-1}$ where $\Sigma_{y}=$ $\sigma_{y} \otimes \sigma_{0}$ with $\sigma_{0}$ being $2 \times 2$ unit matrix. In order to find the eigenenergies of this Hamiltonian analytically, as before, it is convenient to perform a unitary transformation $\tilde{\mathrm{H}}=\mathrm{UHU}^{\dagger}$ with unitary operator $\mathrm{U}=\mathrm{e}^{i \sigma_{z} \phi} \mathrm{e}^{i s_{z} \phi / 2}$ where $\sigma$ and $s$ act on pseudo-spin and real spin respectively. With this transformation, the 
eigenvalue relation for valley $K$ has the form $\tilde{H} \Psi(r, \phi)=E \Psi(r, \phi)$ where $\psi=$ $\mathrm{U} \Psi(\mathrm{r}, \phi)$ and

$$
\begin{aligned}
& \tilde{\mathcal{H}}=\frac{\hbar^{2}}{2 M}\left[\left(\partial_{\mathrm{r}}+\frac{l_{z}+\frac{\Phi}{\Phi_{0}}-\sigma_{z}-\frac{s_{z}}{2}+1}{r}\right)\left(\partial_{\mathrm{r}}+\frac{l_{z}+\frac{\Phi}{\Phi_{0}}-\sigma_{z}-\frac{s_{z}}{2}+2}{r}\right) \sigma_{+}\right. \\
& \left.+\left(-\partial_{\mathrm{r}}+\frac{l_{z}+\frac{\Phi}{\Phi_{0}}-\sigma_{z}-\frac{s_{z}}{2}-1}{r}\right)\left(-\partial_{\mathrm{r}}+\frac{l_{z}+\frac{\Phi}{\Phi_{0}}-\sigma_{z}-\frac{s_{z}}{2}-2}{r}\right) \sigma_{-}\right]+V \sigma_{z} \\
& -v_{\mathrm{B}}\left[\left(-\partial_{\mathrm{r}}+\frac{l_{z}+\frac{\Phi}{\Phi_{0}}-\sigma_{z}-\frac{s_{z}}{2}-1}{\mathrm{r}}\right) \sigma_{-} s_{+}+\left(\partial_{\mathrm{r}}+\frac{l_{z}+\frac{\Phi}{\Phi_{0}}-\sigma_{z}-\frac{s_{z}}{2}+1}{r}\right) \sigma_{+} s_{-}\right] .
\end{aligned}
$$

in which we have used relations for polar form of operators $\pi$ and $\pi^{\dagger}$ presented in Eq. 131.

One can separate the radial and angular parts of the wave function by using the ansatz $\Psi(r, \phi)=e^{i m \phi} \chi_{m}(r)$, where $\chi=\left(\chi_{A}^{* \uparrow}, \chi_{A}^{* \downarrow}, \chi_{B}^{* \uparrow}, \chi_{B}^{* \downarrow}\right)$ is a four component spinor, and rewrite the relation $\tilde{H} \Psi(r, \phi)=E \Psi(r, \phi)$ as four coupled differential equations

$$
\left\{\begin{array}{l}
\frac{\hbar^{2}}{2 M}\left(\partial_{r}+\frac{\bar{m}-\frac{1}{2}}{r}\right)\left(\partial_{r}+\frac{\bar{m}+\frac{1}{2}}{r}\right) \chi_{B}^{\uparrow}=(E-V) \chi_{A^{\prime}}^{\uparrow} \\
\frac{\hbar^{2}}{2 M}\left(\partial_{r}+\frac{\bar{m}+\frac{1}{2}}{r}\right)\left(\partial_{r}+\frac{\bar{m}+\frac{3}{2}}{r}\right) \chi_{B}^{\downarrow}-v_{B}\left(\partial_{r}+\frac{\bar{m}+\frac{1}{2}}{r}\right) \chi_{B}^{\uparrow}=(E-V) \chi_{A^{\prime}}^{\downarrow} \\
\frac{\hbar^{2}}{2 M}\left(-\partial_{r}+\frac{\bar{m}-\frac{1}{2}}{r}\right)\left(-\partial_{r}-\frac{\bar{m}-\frac{3}{2}}{r}\right) \chi_{A}^{\uparrow}-v_{B}\left(-\partial_{r}+\frac{\bar{m}-\frac{1}{2}}{r}\right) \chi_{A}^{\downarrow}=(E+V) \chi_{B^{\prime}}^{\uparrow} \\
\frac{\hbar^{2}}{2 M}\left(-\partial_{r}+\frac{\bar{m}+\frac{1}{2}}{r}\right)\left(-\partial_{r}+\frac{\bar{m}-\frac{1}{2}}{r}\right) \chi_{A}^{\downarrow}=(E+V) \chi_{B}^{\downarrow} .
\end{array}\right.
$$

For valley $\mathrm{K}^{\prime}$ the system of equations from which eigenenergies can be found is given by

$$
\left\{\begin{array}{l}
-\frac{\hbar^{2}}{2 M}\left(-\partial_{r}+\frac{\bar{m}+\frac{1}{2}}{r}\right)\left(-\partial_{r}+\frac{\bar{m}-\frac{1}{2}}{r}\right) \chi_{B}^{\uparrow}+\nu_{B}\left(-\partial_{r}+\frac{\bar{m}-\frac{1}{2}}{r}\right) \chi_{B}^{\downarrow}=(E+V) \chi_{A^{\prime}}^{\uparrow} \\
-\frac{\hbar^{2}}{2 M}\left(-\partial_{r}+\frac{\bar{m}-\frac{1}{2}}{r}\right)\left(-\partial_{r}+\frac{\bar{m}-\frac{3}{2}}{r}\right) \chi_{B}^{\downarrow}=(E+V) \chi_{A^{\prime}}^{\downarrow} \\
-\frac{\hbar^{2}}{2 M}\left(\partial_{r}+\frac{\bar{m}+\frac{1}{2}}{r}\right)\left(\partial_{r}+\frac{\bar{m}+\frac{3}{2}}{r}\right) \chi_{A}^{\uparrow}=(E-V) \chi_{B^{\prime}}^{\uparrow} \\
-\frac{\hbar^{2}}{2 M}\left(\partial_{r}+\frac{\bar{m}-\frac{1}{2}}{r}\right)\left(\partial_{r}+\frac{\bar{m}+\frac{1}{2}}{r}\right) \chi_{A}^{\downarrow}+v_{B}\left(\partial_{r}+\frac{\bar{m}+\frac{1}{2}}{r}\right) \chi_{A}^{\uparrow}=(E-V) \chi_{B}^{\downarrow} .
\end{array}\right.
$$


For regions with $V \neq 0$ the four linearly independent solutions of Eq. 160 are $J_{m}\left(e^{+3 i \pi / 4} k r\right), J_{m}\left(e^{-3 i \pi / 4} k r\right)$ which are well-defined at origin and $K_{m}\left(e^{+i \pi / 4} k r\right)$ and $K_{m}\left(e^{-i \pi / 4} k r\right)$, which decay as $r \rightarrow \infty$. So for region $\mathrm{I}(0<r<R-W / 2)$ the solution of Eq. 160 is

$$
\chi(r)=\left(\begin{array}{c}
\alpha_{1} J_{m-3 / 2}\left(e^{ \pm 3 i \pi / 4} k r\right) \\
\alpha_{2} J_{m-1 / 2}\left(e^{ \pm 3 i \pi / 4} k r\right) \\
\alpha_{3} J_{m+1 / 2}\left(e^{ \pm 3 i \pi / 4} k r\right) \\
\alpha_{4} J_{m+3 / 2}\left(e^{ \pm 3 i \pi / 4} k r\right)
\end{array}\right)
$$

By using this spinor as the solution of Eq. 160, we reach to a matrix equation

$$
\left(\begin{array}{cccc}
V & 0 & \mp \frac{i k^{2}}{2 M} & 0 \\
0 & V & -e^{ \pm 3 i \pi / 4} k v_{B} & \mp \frac{i k^{2}}{2 M} \\
\mp \frac{i k^{2}}{2 M} & -e^{ \pm 3 i \pi / 4} k v_{B} & -V & 0 \\
0 & \mp \frac{i k^{2}}{2 M} & 0 & -V
\end{array}\right)\left(\begin{array}{c}
\alpha_{1} \\
\alpha_{2} \\
\alpha_{3} \\
\alpha_{4}
\end{array}\right)=E\left(\begin{array}{c}
\alpha_{1} \\
\alpha_{2} \\
\alpha_{3} \\
\alpha_{4}
\end{array}\right),
$$

from which one can find the wave vectors $k$ and coefficients $\alpha_{i}(i=1,2,3,4)$ for each wave vector. So the radial part of wave function $\Psi(r, \phi)$ in region one is given by

$$
\begin{gathered}
x_{1}=A_{1}\left(\begin{array}{c}
a_{1} J_{m-3 / 2}\left(e^{3 i \pi / 4} k_{1} r\right) \\
a_{2} J_{m-1 / 2}\left(e^{3 i \pi / 4} k_{1} r\right) \\
a_{3} J_{m+1 / 2}\left(e^{3 i \pi / 4} k_{1} r\right) \\
a_{4} J_{m+3 / 2}\left(e^{3 i \pi / 4} k_{1} r\right)
\end{array}\right)+A_{2}\left(\begin{array}{c}
b_{1} J_{m-3 / 2}\left(e^{3 i \pi / 4} k_{2} r\right) \\
b_{2} J_{m-1 / 2}\left(e^{3 i \pi / 4} k_{2} r\right) \\
b_{3} J_{m+1 / 2}\left(e^{3 i \pi / 4} k_{2} r\right) \\
b_{4} J_{m+3 / 2}\left(e^{3 i \pi / 4} k_{2} r\right)
\end{array}\right) \\
+A_{3}\left(\begin{array}{c}
c_{1} J_{m-3 / 2}\left(e^{-3 i \pi / 4} k_{1} r\right) \\
c_{2} J_{m-1 / 2}\left(e^{-3 i \pi / 4} k_{1} r\right) \\
c_{3} J_{m+1 / 2}\left(e^{-3 i \pi / 4} k_{1} r\right) \\
c_{4} J_{m+3 / 2}\left(e^{-3 i \pi / 4} k_{1} r\right)
\end{array}\right)+A_{4}\left(\begin{array}{c}
d_{1} J_{m-3 / 2}\left(e^{-3 i \pi / 4} k_{2} r\right) \\
d_{2} J_{m-1 / 2}\left(e^{-3 i \pi / 4} k_{2} r\right) \\
d_{3} J_{m+1 / 2}\left(e^{-3 i \pi / 4} k_{2} r\right) \\
d_{4} J_{m+3 / 2}\left(e^{-3 i \pi / 4} k_{2} r\right)
\end{array}\right),
\end{gathered}
$$

where we have chosen those wave vectors $\left(k_{1}\right.$ and $\left.k_{2}\right)$ that when $v_{\mathrm{B}} \rightarrow 0$ are given by $k=\sqrt{\frac{2 M}{\hbar^{2}}}\left(V^{2}-E^{2}\right)^{1 / 4}$. 

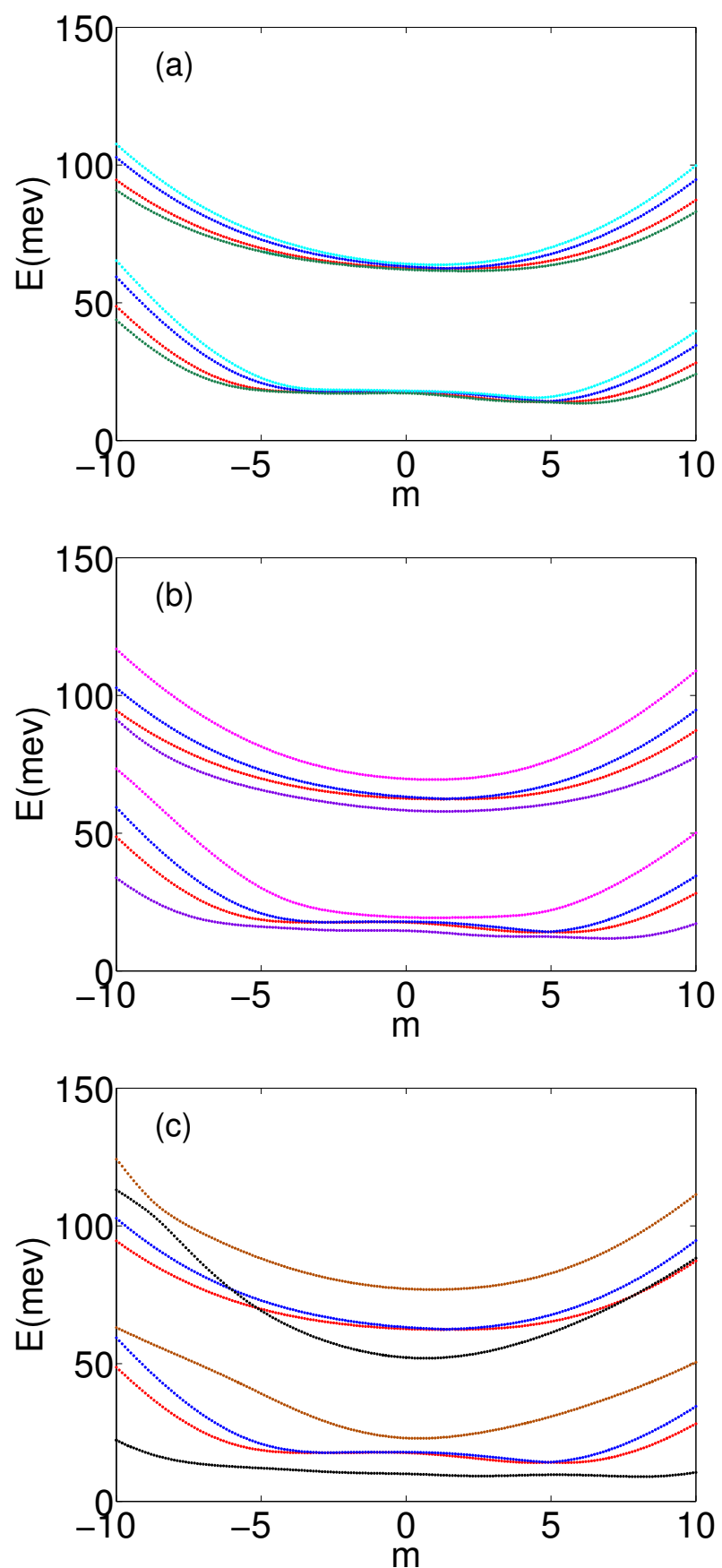

Figure 28: The lowest energy levels of a BLGR (valley $\mathrm{K}$ ) with radius $\mathrm{R}=5 \mathrm{O} \mathrm{nm}$ and width $\mathrm{W}=\mathbf{2 0} \mathrm{nm}$ as a function of $\mathrm{m}$ for different values of Rashba SOC $\bar{v}_{\mathrm{B}}$. The red and blue curves corresponds to the case $\bar{v}_{\mathrm{B}}=0$ and they come from applying the unitary transformation U. Rashba SOC strength for panel (a) is $\bar{v}_{\mathrm{B}}=0.01$, panel (b) $\bar{v}_{\mathrm{B}}=0.05$ and for panel (c) $\bar{v}_{\mathrm{B}}=0.1$. 
Similarly for region III $(r>R+W / 2)$, we choose well-defined functions at infinity and the wave function reads as

$$
\begin{gathered}
\chi_{3}=A_{13}\left(\begin{array}{c}
a_{1}^{\prime} k_{m-3 / 2}\left(e^{i \pi / 4} k_{1}^{\prime} r\right) \\
a_{2}^{\prime} k_{m-1 / 2}\left(e^{i \pi / 4} k_{1}^{\prime} r\right) \\
a_{3}^{\prime} k_{m+1 / 2}\left(e^{i \pi / 4} k_{1}^{\prime} r\right) \\
a_{4}^{\prime} k_{m+3 / 2}\left(e^{i \pi / 4} k_{1}^{\prime} r\right)
\end{array}\right)+A_{14}\left(\begin{array}{c}
b_{1}^{\prime} k_{m-3 / 2}\left(e^{i \pi / 4} k_{2}^{\prime} r\right) \\
b_{2}^{\prime} k_{m-1 / 2}\left(e^{i \pi / 4} k_{2}^{\prime} r\right) \\
b_{3}^{\prime} k_{m+1 / 2}\left(e^{i \pi / 4} k_{2}^{\prime} r\right) \\
b_{4}^{\prime} k_{m+3 / 2}\left(e^{i \pi / 4} k_{2}^{\prime} r\right)
\end{array}\right) \\
+A_{15}\left(\begin{array}{c}
c_{1}^{\prime} K_{m-3 / 2}\left(e^{-i \pi / 4} k_{1}^{\prime} r\right) \\
c_{2}^{\prime} K_{m-1 / 2}\left(e^{-i \pi / 4} k_{1}^{\prime} r\right) \\
c_{3} K_{m+1 / 2}\left(e^{-i \pi / 4} k_{1}^{\prime} r\right) \\
c_{4}^{\prime} k_{m+3 / 2}\left(e^{-i \pi / 4} k_{1}^{\prime} r\right)
\end{array}\right)+A_{16}\left(\begin{array}{c}
d_{1}^{\prime} k_{m-3 / 2}\left(e^{-i \pi / 4} k_{2}^{\prime} r\right) \\
d_{2}^{\prime} k_{m-1 / 2}\left(e^{-i \pi / 4} k_{2}^{\prime} r\right) \\
d_{3}^{\prime} k_{m+1 / 2}\left(e^{-i \pi / 4} k_{2}^{\prime} r\right) \\
d_{4}^{\prime} k_{m+3 / 2}\left(e^{-i \pi / 4} k_{2}^{\prime} r\right)
\end{array}\right),
\end{gathered}
$$

where the wave vectors $k_{i}^{\prime}(i=1,2)$ and coefficients can be obtained from matrix equation

$$
\left(\begin{array}{cccc}
V & 0 & \pm \frac{i k^{2}}{2 M} & 0 \\
0 & V & e^{ \pm i \pi / 4} k v_{B} & \pm \frac{i k^{2}}{2 M} \\
\pm \frac{i k^{2}}{2 M} & -e^{ \pm i \pi / 4} k v_{B} & -V & 0 \\
0 & \pm \frac{i k^{2}}{2 M} & 0 & -V
\end{array}\right)\left(\begin{array}{c}
\alpha_{1} \\
\alpha_{2} \\
\alpha_{3} \\
\alpha_{4}
\end{array}\right)=E\left(\begin{array}{c}
\alpha_{1} \\
\alpha_{2} \\
\alpha_{3} \\
\alpha_{4}
\end{array}\right)
$$


For region II $(R-W / 2<r<R+W / 2)$ with $V=0$, the proper solutions are ordinary and modified Bessel functions which lead to

$$
\begin{aligned}
x_{2}=A_{5}\left(\begin{array}{c}
f_{1} J_{m-3 / 2}(k r) \\
f_{2} J_{m-1 / 2}(k r) \\
f_{3} J_{m+1 / 2}(k r) \\
f_{4} J_{m+3 / 2}(k r)
\end{array}\right)+A_{6}\left(\begin{array}{c}
f_{1}^{\prime} J_{m-3 / 2}\left(k^{\prime} r\right) \\
f_{2}^{\prime} J_{m-1 / 2}\left(k^{\prime} r\right) \\
f_{3}^{\prime} J_{m+1 / 2}\left(k^{\prime} r\right) \\
f_{4}^{\prime} J_{m+3 / 2}\left(k^{\prime} r\right)
\end{array}\right)+A_{7}\left(\begin{array}{l}
g_{1} Y_{m-3 / 2}(k r) \\
g_{2} Y_{m-1 / 2}(k r) \\
g_{3} Y_{m+1 / 2}(k r) \\
g_{4} Y_{m+3 / 2}(k r)
\end{array}\right) \\
+A_{8}\left(\begin{array}{c}
g_{1}^{\prime} Y_{m-3 / 2}\left(k^{\prime} r\right) \\
g_{2}^{\prime} Y_{m-1 / 2}\left(k^{\prime} r\right) \\
g_{1}^{\prime} Y_{m+1 / 2}\left(k^{\prime} r\right) \\
g_{1}^{\prime} Y_{m+3 / 2}\left(k^{\prime} r\right)
\end{array}\right)+A_{9}\left(\begin{array}{c}
h_{1} K_{m-3 / 2}(q r) \\
h_{2} K_{m-1 / 2}(q r) \\
h_{3} K_{m+1 / 2}(q r) \\
h_{4} K_{m+3 / 2}(q r)
\end{array}\right)+A_{10}\left(\begin{array}{l}
h_{1}^{\prime} K_{m-3 / 2}\left(q^{\prime} r\right) \\
h_{2}^{\prime} K_{m-1 / 2}\left(q^{\prime} r\right) \\
h_{3}^{\prime} K_{m+1 / 2}\left(q^{\prime} r\right) \\
h_{4}^{\prime} K_{m+3 / 2}\left(q^{\prime} r\right)
\end{array}\right) \\
+A_{11}\left(\begin{array}{c}
t_{1} I_{m-3 / 2}(q r) \\
t_{2} I_{m-1 / 2}(q r) \\
t_{3} I_{m+1 / 2}(q r) \\
t_{4} I_{m+3 / 2}(q r)
\end{array}\right)+A_{12}\left(\begin{array}{c}
t_{2}^{\prime} I_{m-1 / 2}\left(q^{\prime} r\right) \\
t_{3}^{\prime} I_{m+1 / 2}\left(q^{\prime} r\right) \\
t_{4}^{\prime} I_{m+3 / 2}\left(q^{\prime} r\right)
\end{array}\right) .
\end{aligned}
$$

where wave vectors $k$ and $k^{\prime}$ and their corresponding coefficients are obtained from

$$
\left(\begin{array}{cccc}
0 & 0 & \frac{k^{2}}{2 M} & 0 \\
0 & 0 & -k v_{B} & \frac{k^{2}}{2 M} \\
\frac{k^{2}}{2 M} & -k \nu_{B} & 0 & 0 \\
0 & \mp \frac{k^{2}}{2 M} & 0 & 0
\end{array}\right)\left(\begin{array}{c}
\alpha_{1} \\
\alpha_{2} \\
\alpha_{3} \\
\alpha_{4}
\end{array}\right)=E\left(\begin{array}{c}
\alpha_{1} \\
\alpha_{2} \\
\alpha_{3} \\
\alpha_{4}
\end{array}\right),
$$

while for wave vectors $q$ and $q^{\prime}$ the matrix equation is

$$
\left(\begin{array}{cccc}
0 & 0 & \frac{k^{2}}{2 M} & 0 \\
0 & 0 & k v_{B} & \frac{k^{2}}{2 M} \\
\frac{k^{2}}{2 M} & -k v_{B} & 0 & 0 \\
0 & \mp \frac{k^{2}}{2 M} & 0 & 0
\end{array}\right)\left(\begin{array}{l}
\beta_{1} \\
\beta_{2} \\
\beta_{3} \\
\beta_{4}
\end{array}\right)=E\left(\begin{array}{l}
\beta_{1} \\
\beta_{2} \\
\beta_{3} \\
\beta_{4}
\end{array}\right) .
$$

We have found the proper wave vectors in region II by using this fact that when $v_{\mathrm{B}} \rightarrow 0$, the wave vectors should be equal to the case when Rashba SOC is absent, 
that is $k=\sqrt{\frac{2 M}{\hbar^{2}}|E|}$. The proper boundary conditions are, as before, the continuity of the wave functions $(\Psi(r, \phi))$ at the interfaces $R-\frac{W}{2}$ and $R+\frac{W}{2}$

$$
\left\{\begin{array}{l}
\left.\Psi_{1}\right|_{\mathrm{r}=\mathrm{R}-\frac{\mathrm{W}}{2}}=\left.\Psi_{2}\right|_{\mathrm{r}=\mathrm{R}-\frac{\mathrm{W}}{2}} \\
\left.\Psi_{2}\right|_{\mathrm{r}=\mathrm{R}+\frac{\mathrm{W}}{2}}=\left.\Psi_{3}\right|_{\mathrm{r}=\mathrm{R}+\frac{\mathrm{W}}{2}},
\end{array}\right.
$$

and probability flux

$$
\left\{\begin{array}{l}
\left.\tilde{v}_{\mathrm{r}} \Psi_{1}\right|_{\mathrm{r}=\mathrm{R}-\frac{\mathrm{W}}{2}}=\left.\tilde{v}_{\mathrm{r}} \Psi_{2}\right|_{\mathrm{r}=\mathrm{R}-\frac{\mathrm{W}}{2}}, \\
\left.\tilde{v}_{\mathrm{r}} \Psi_{2}\right|_{\mathrm{r}=\mathrm{R}+\frac{\mathrm{W}}{2}}=\left.\tilde{v}_{\mathrm{r}} \Psi_{3}\right|_{\mathrm{r}=\mathrm{R}+\frac{\mathrm{W}}{2}},
\end{array}\right.
$$

in which the velocity operator is defined as (See Sec. 2.4.1.)

$$
\begin{aligned}
\tilde{v}_{\mathrm{r}}= & \frac{i \hbar}{M}\left[\left(\partial_{\mathrm{r}}+\frac{l_{z}+\frac{s_{z}}{2}+1}{r}\right) \sigma_{+}-\left(-\partial_{\mathrm{r}}+\frac{l_{z}+\frac{s_{z}}{2}-1}{r}\right) \sigma_{-}\right] \\
& +i v_{\mathrm{B}}\left[\sigma_{+} s_{-}-\sigma_{-} s_{+}\right] .
\end{aligned}
$$

These conditions lead to a system of equations from which energy eigenvalues of a BLGR in the presence of Rashba SOC can be obtained. The energy eigenvalues of a BLGR with radius $R=50 \mathrm{~nm}$ and width $W=20 \mathrm{~nm}$ as a function of $\mathrm{m}$ is shown in Fig. 28 for different strengths of Rashba SOC $\bar{v}_{B}=\frac{2 M a}{\hbar^{2}} v_{B}$ where $a$ is the graphene lattice constant. One should notice that as a consequence of using the unitary transformation $\mathrm{U}=e^{-\mathrm{i} \sigma_{z} \phi} e^{i s_{z} \phi / 2}$ we obtain a split in the spectrum of the BLGR which is regardless of Rashba SOC. This splitting is such that states with $\mathrm{m}^{\prime}=\mathrm{m} \pm 1$ are having the same energy in the absence of Rashba SOC $\left(\bar{v}_{\mathrm{B}} \neq 0\right)$. This splitting which comes only because of transformation $U$ are shown by blue and red curves in Fig. 28. The effect of Rashba SOC on the two lowest energy levels can be understood by comparing the energy levels to blue and red curves. When the Rashba SOC strength is weak only the states with larger values $|\mathrm{m}|$ are affected by Rashba SOC (panel a). However the splitting becomes appreciable even for small values of $|\mathrm{m}|$ as $\bar{v}_{\mathrm{B}}$ increases. It is also notable that Rashba SOC tends to flatten the Mexican hat form of the lowest level in BLGR (panel b, c). In Fig. 28 we have only plotted the positive energy levels. The negative part of the energy 

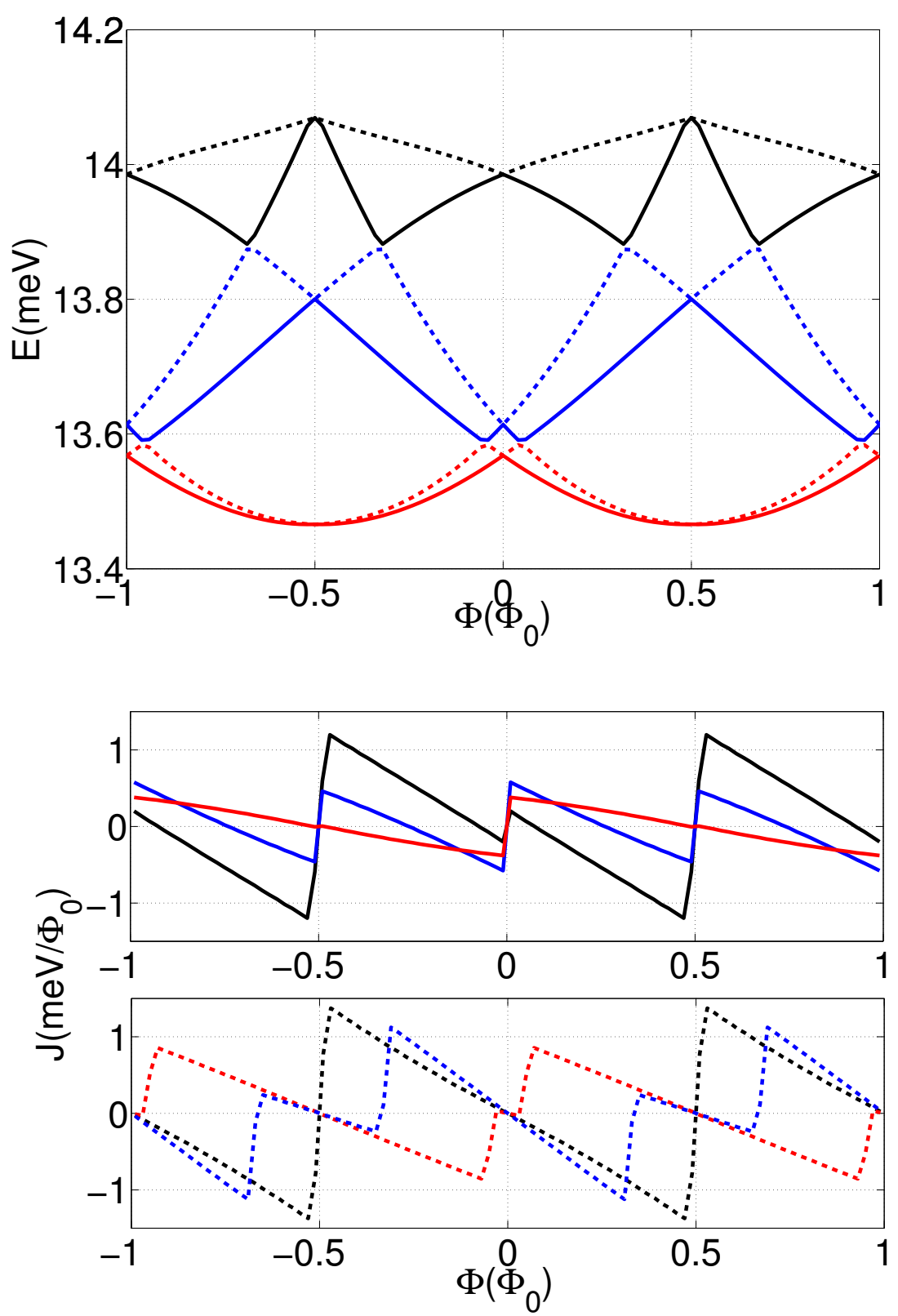

Figure 29: Top panel: lowest energy levels of a BLGR in the presence of Rashba SOC. Each single energy level is shown by solid or dashed curves with different colors. Bottom panel: persistent current as a function of magnetic flux considering both valleys in the presence of Rashba SOC with strength $\bar{v}_{\mathrm{B}}=0.02$. Persistent current with different colors and line styles corresponds to the energy levels occupied shown in the left panels. 

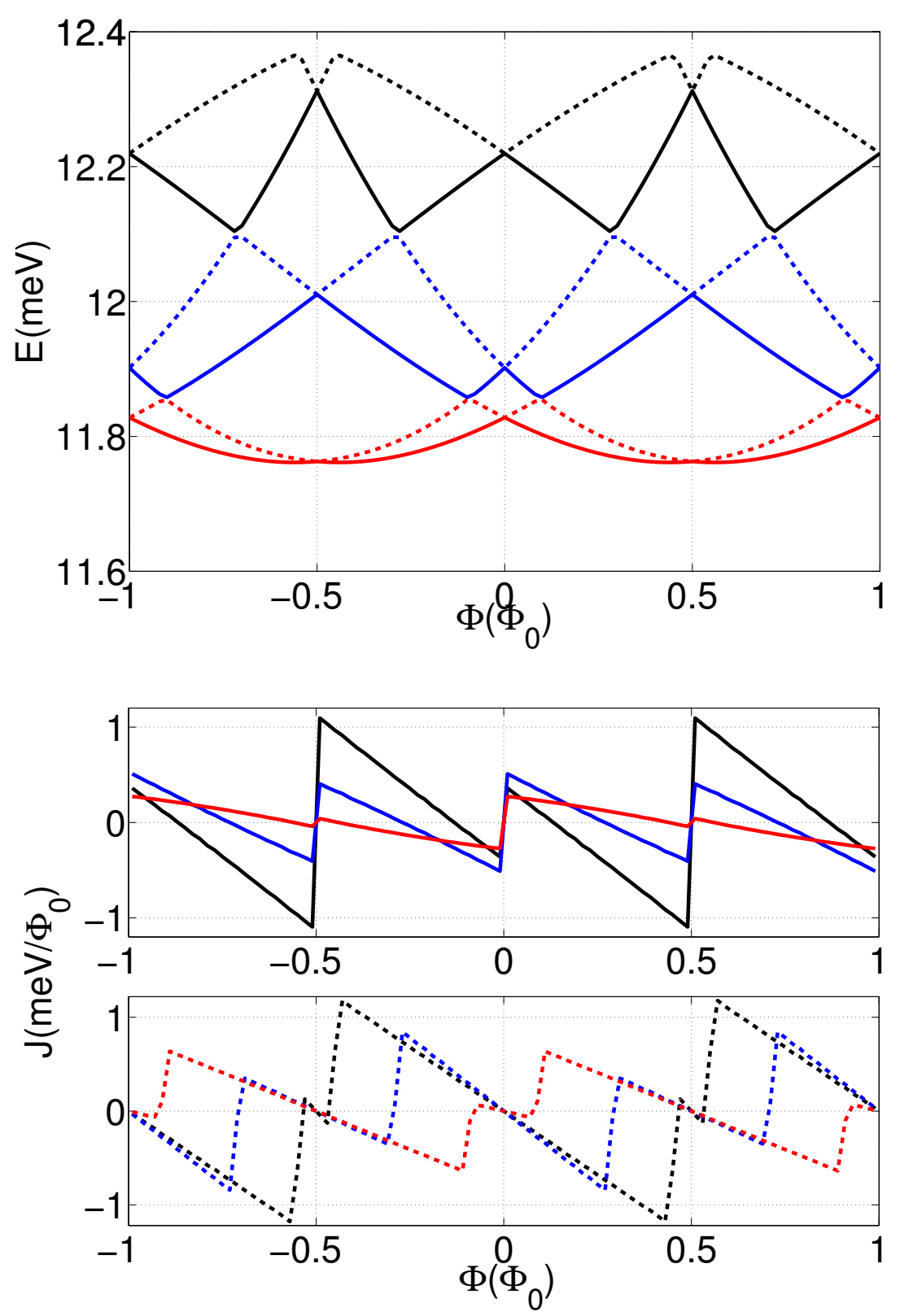

Figure 30: Top panel: lowest energy levels of a BLGR in the presence of Rashba SOC. Each single energy level is shown by solid or dashed curves with different colors. Bottom panel: persistent current as a function of magnetic flux considering both valleys in the presence of Rashba SOC with strength $\bar{v}_{\mathrm{B}}=0.05$. Persistent current with different colors and line styles corresponds to the energy levels occupied shown in the left panels. 
spectrum can be obtained by using relation $E^{\tau}(\bar{m})=-E^{\tau}(-\bar{m})$ for each valley, and also energy levels in different valleys are related by $E^{\tau}(\bar{m})=-E^{-\tau}(-\bar{m})$ which can be understood by comparing Eq. 159 with Eq. 160.

In the upper panel of Fig. 29 and Fig. 30 the lowest energy levels with Rashba SOC $\bar{v}_{\mathrm{B}}=0.02$ and $\bar{v}_{\mathrm{B}}=0.05$ considering both valleys as a function of magnetic field are shown. The PC when only the first lowest level $(N=1)$ is occupied has a kink at $\Phi=0$. Compared to the PC in Fig. 27 with $N=1$, it is like that it has been shifted by $\Phi_{0} / 2$. Since the spin degeneracy is lifted due to Rashba SOC and energy levels in Fig. 29 and Fig. 30 are single levels, the PC with $N=2$ is no longer twice the PC with $N=1$ and actually the structure of the PC has kinks located at different values of $\Phi$. It is notable that for filling numbers $N>1$ when single energy levels touch each other there are no kinks in the PC.

The magnitude of PC has decreased compared to the case without Rashba SOC since Rashba SOC tends to flatten the lowest energy levels and this will decrease the slope of the levels which results in reduction of PC. Increasing strength of Rashba SOC tends to increase the energy level splitting and it results in changing the number of kinks in the structure of PC specially for even number of electrons in the ring. For example, $\mathrm{PC}$ in Fig. 30 with $\bar{v}_{\mathrm{B}}=0.05$ for $\mathrm{N}=2$ and $\mathrm{N}=6$ has additional kinks located at $\Phi= \pm \phi_{0}$ compared to the case when $\bar{v}_{\mathrm{B}}=0.02$ in Fig. 29.

\subsubsection{Calculating the velocity operator in the presence of Rashba SOC}

In order to find the radial component of the velocity operator when the ring is subjected to Rashba SOC, we start by deriving velocity operators in $x$ and $y$ directions

$$
\begin{aligned}
& \hat{v}_{x}=\frac{i}{\hbar}[\mathcal{H}, x]=\frac{-1}{M}\left(\pi_{+} \sigma_{+}+\pi_{-} \sigma_{-}\right)+i v_{B}\left(\sigma_{+} s_{-}-\sigma_{-} s_{+}\right) \\
& \hat{v}_{y}=\frac{i}{\hbar}[\mathcal{H}, y]=\frac{i}{M}\left(\pi_{+} \sigma_{+}-\pi_{-} \sigma_{-}\right)+v_{\mathrm{B}}\left(\sigma_{+} s_{-}+\sigma_{-} s_{+}\right),
\end{aligned}
$$


where $\mathcal{H}$ is the Hamiltonian of the BLGR in the presence of Rashba SOC. One can write the radial component of the velocity operator $\hat{v}_{r}=\hat{v}_{x} \cos (\phi)+\hat{v}_{y} \sin (\phi)$ as

$$
\hat{v}_{\mathrm{r}}=\frac{-1}{M}\left(e^{-i \phi} \pi_{+} \sigma_{+}+e^{i \phi} \pi_{-} \sigma_{-}\right)+i v_{\mathrm{B}}\left(e^{-i \phi} \sigma_{+} s_{-}-e^{i \phi} \sigma_{-} s_{+}\right) .
$$

Using the polar definition of $\pi_{ \pm}=\mp i \hbar e^{\mp i \phi}\left( \pm \partial_{\mathrm{r}}+\frac{\mathrm{l}_{z}}{\mathrm{r}}\right)$

$$
\begin{aligned}
\hat{v}_{\mathrm{r}}= & \frac{-1}{M} \times\left(-i \hbar\left(\partial_{\mathrm{r}}+\frac{l_{z}+2}{r}\right) e^{-2 i \phi} \sigma_{+}+i \hbar\left(-\partial_{\mathrm{r}}+\frac{l_{z}-2}{r}\right) e^{2 i \phi} \sigma_{-}\right) \\
& +i v_{\mathrm{B}}\left(e^{-i \phi} \sigma_{+} s_{-}-e^{i \phi} \sigma_{-} s_{+}\right),
\end{aligned}
$$

To transform the velocity operator, we use the same ansatz that we applied to the Hamiltonian of the system, that is

$$
\tilde{v}_{\mathrm{r}}=e^{i \sigma_{z} \phi} e^{i s_{z} \phi / 2} \hat{v}_{\mathrm{r}} e^{-i \sigma_{z} \phi} e^{-i s_{z} \phi / 2}
$$

and using the following relations

$$
\begin{aligned}
& e^{i s_{z} \phi / 2} s_{ \pm} e^{-i s_{z} \phi / 2}=s_{ \pm} e^{ \pm i \phi} \\
& e^{i \sigma_{z} \phi} \sigma_{ \pm} e^{-i \sigma_{z} \phi}=\sigma_{ \pm} e^{ \pm 2 i \phi}
\end{aligned}
$$

the radial component of velocity operator corresponding to transferred Hamiltonian $\tilde{\mathcal{H}}$ read as

$$
\begin{aligned}
\tilde{v}_{\mathrm{r}}= & \frac{i \hbar}{M}\left[\left(\partial_{\mathrm{r}}+\frac{l_{z}+\frac{s_{z}}{2}+1}{r}\right) \sigma_{+}-\left(-\partial_{\mathrm{r}}+\frac{l_{z}+\frac{s_{z}}{2}-1}{\mathrm{r}}\right) \sigma_{-}\right] \\
& +i v_{\mathrm{B}}\left[\sigma_{+} s_{-}-\sigma_{-} s_{+}\right] .
\end{aligned}
$$

\subsection{EXPERIMENTAL REALIZATION OF GRAPHENE RINGS}

Metal rings were the first quantum rings fabricated to investigate quantum coherence effects (Webb et al., 1985; Stone and Imry, 1986). Later, thanks to advances 
in nano fabrication technology, rings from semiconductor heterostructures could be prepared (Timp et al., 1987; K. Ismail and Lee, 1991). In monolayer graphene, lithographic cutting is one way to create a ring which is equivalent to applying an infinite mass boundary condition in theoretical studies (Berry and Mondragon, 1987; Recher et al., 2007). Fabricating a ring through cutting always leaves us with edge impurities which may influence electrical properties, for example by suppressing the measured PC (S. Russo and Sobhani, 2008; Huefner et al., 2010). It has been shown theoretically that not only edge disorders can affect the spectrum of the system but specific types of edge states such as zigzag or arm chair also can influence electrical properties of a graphene ring (D. A. Bahamon and Schulz, 2009). Also there have been several theoretical studies on MLGRs using the tight-binding method studying edge disorder (T. Luo et al., 2009; Wurm et al., 2010; Rycerz and Beenakker, 2007).

Using electrostatic confinement one can dispose of problems which occurred due to edge disorders of the lithographic cutting. However there are still some challenges. One challenge comes from the fabricating of the gates and how perfectly one can create a ring-shaped gate. The other limit is that we can not have a smooth circular boundary because of the underlying crystal structure which is rugged on the scale of the atomic length.

\subsection{SUMMARY AND CONCLUSIONS}

In the present chapter we have analyzed the electronic properties of a bilayer graphene ring with finite width using a two-band and four-band Hamiltonian. We have also investigated the ABoscillations in a closed bilayer graphene ring through calculating the persistent current. We have shown that confining electrons in a ring shape can break the time reversal symmetry in a single valley and by applying a magnetic flux one can control the valley degeneracy breaking. Valley degeneracy breaking in graphene based devices can be used in so called valleytronics applications. Furthermore, we have calculated the effect of Rashba 
spin-orbit coupling on the energy levels in a bilayer graphene ring analytically. We have shown that in weak Rashba spin-orbit coupling there is a splitting in the energy eigenstates with large value of $|\mathrm{m}|$. However the energy states with small $|\mathrm{m}|$ are hardly influenced by weak Rashba spin-orbit coupling. When the strength of the Rashba spin-orbit coupling increases all the states are affected which tends to flatten the Mexican hat structure of the lowest energy level as a function of angular momentum eigenvalue and this can manifest itself by reducing the persistent current as the number of particles in the ring increases. Rashba spin-orbit coupling can also lead to having extra kinks in the structure of persistent current due to energy level spin splitting. 



\subsection{INTRODUCTION AND MOTIVATION}

By using an invariant expansion of the trigonal band structure of graphene, Winkler and Zülicke (2010) showed that an in-plane electric field in graphene can induce a novel effect which couples the orbital motion of an electron with the pseudospin degree of freedom, which is called orbital-Rashba effect and has the form

$$
H_{O R}=V_{O R}\left(k_{x} E_{y}-k_{y} E_{x}\right) \sigma_{z}
$$

where the coefficient $V_{O R}$ shows the strength of this effect. However its value can not be obtained from a symmetry analysis, therefore we suppose here that this coefficient is small enough to make this term very small in comparison with the effect of an electric field on the orbital motion of electrons.

As the first step in this chapter, by using the same method as applied by Peres and Castro (2007), we try to study the effect of orbital-Rashba on LLs! of graphene. The Hamiltonian of graphene in the presence of crossed uniform electric and magnetic field has the form

$$
\mathrm{H}=\mathrm{H}_{\mathrm{D}}+\mathrm{H}_{\mathrm{B}}+\mathrm{H}_{\mathrm{OR}}=\hbar v_{\mathrm{f}} \boldsymbol{\sigma} \cdot \boldsymbol{\Pi}-\mathrm{e} \boldsymbol{E} \cdot \boldsymbol{r} \mathbb{1}+\mathrm{V}_{\mathrm{OR}}(\boldsymbol{k} \times \boldsymbol{E}) \cdot \hat{z} \sigma_{\mathrm{z}},
$$

where the term $H_{D}$ is the Hamiltonian of massless Dirac Fermions in graphene in the presence of perpendicular magnetic field. The term $\mathrm{H}_{B}$ shows the effect of an electric field on the orbital motion of electrons, and the term $\mathrm{H}_{\mathrm{OR}}$ is due to the orbital-Rashba effect. Here $\mathbb{1}$ denotes a unit matrix in pseudospin space.

By having the transverse electric field only in $y$ direction and using the bosonic operators $a$ and $a^{\dagger}$, the Hamiltonian reads as

$$
H=E_{f}\left(a^{\dagger} \sigma_{-}+a \sigma_{+}\right)+E_{B}\left(a+a^{\dagger}\right) \mathbb{1}-e E l_{B}^{2} k \mathbb{1}+\Delta_{R}\left(a+a^{\dagger}\right) \sigma_{z},
$$


where $E_{f}=\frac{\sqrt{2} \hbar v_{f}}{l_{B}}, E_{B}=\frac{e E l_{B}}{\sqrt{2}}$ and $\Delta_{R}=\frac{V_{O R} E_{B}}{e l_{B}^{2}}$, in which $E$ is the electric field, $e$ the electron charge and $l_{B}=\sqrt{\frac{\hbar}{e B}}$ the magnetic length.

Peres and Castro (2007) found an analytical method to solve the energy eigenvalue in Eq. 180 without orbital Rashba term $\left(\Delta_{R}=0\right)$, and the result was the collapse of LLs!. However adding the orbital Rashba term to these two terms makes it difficult to find an exact analytical solution for it. In this chapter, we try to investigate the effect of the orbital-Rashba term on LLs! using perturbation theory.

\subsubsection{Graphene in a transverse electric and perpendicular magnetic fields}

In the present section, we briefly discuss the method Peres and Castro (2007) used in their calculations to find the eigenstates and eigenenergies of Dirac fermions in the presence of crossed uniform electric and magnetic fields. Here we assume that $\Delta_{R}$ in Hamiltonian (179) is zero. Instead of finding the energy eigenvalues of the Hamiltonian

$$
H=\left(\begin{array}{cc}
E_{B}\left(a+a^{\dagger}\right)-e E l_{B}^{2} k & E_{f} a \\
E_{f} a^{\dagger} & E_{B}\left(a+a^{\dagger}\right)-e E l_{B}^{2} k
\end{array}\right),
$$

one can solve the eigenvalue equation

$$
\left(\begin{array}{cc}
E_{B}\left(a+a^{\dagger}\right) & E_{f} a \\
E_{f} a^{\dagger} & E_{B}\left(a+a^{\dagger}\right)
\end{array}\right)\left(\begin{array}{c}
\left|\psi_{1}\right\rangle \\
\left|\psi_{2}\right\rangle
\end{array}\right)=\epsilon_{0}\left(\begin{array}{l}
\left|\psi_{1}\right\rangle \\
\left|\psi_{2}\right\rangle
\end{array}\right),
$$

where $\epsilon_{0}=\epsilon+e E l_{\mathrm{B}}^{2} k$, and the effective Hamiltonian has the form

$$
\tilde{\mathcal{H}}=\left(\begin{array}{cc}
E_{B}\left(a+a^{\dagger}\right) & E_{f} a \\
E_{f} a^{\dagger} & E_{B}\left(a+a^{\dagger}\right)
\end{array}\right) .
$$

In order to diagonalize this effective Hamiltonian, one can define a new Hamiltonian as

$$
\overline{\mathcal{H}} \equiv \sigma_{z} \tilde{\mathcal{H}} \sigma_{z}=\left(\begin{array}{cc}
E_{B}\left(a+a^{\dagger}\right) & -E_{f} a \\
-E_{f} a^{\dagger} & E_{B}\left(a+a^{\dagger}\right)
\end{array}\right)
$$


Here the idea is to find a particular linear combination of $\tilde{\mathcal{H}}+\overline{\mathcal{H}}$ and $\overline{\mathcal{H}} \tilde{\mathcal{H}}$ which can help us to solve our actual eigenvalue problem i.e. $\tilde{\mathcal{H}}|\psi\rangle=\epsilon_{0}|\psi\rangle$. These two terms are given by

$$
\begin{aligned}
\overline{\mathrm{H}}+\tilde{\mathrm{H}}= & \left(\begin{array}{cc}
2 \mathrm{E}_{\mathrm{B}}\left(\mathrm{a}+\mathrm{a}^{\dagger}\right) & 0 \\
0 & 2 \mathrm{E}_{\mathrm{B}}\left(\mathrm{a}+\mathrm{a}^{\dagger}\right)
\end{array}\right) \\
& =2 \mathrm{E}_{\mathrm{B}} \mathbb{1}\left(\mathrm{a}+\mathrm{a}^{\dagger}\right)
\end{aligned}
$$

and

$\bar{H} \tilde{H}=\left(\begin{array}{cc}E_{B}^{2}\left(a^{2}+a^{\dagger^{2}}\right)+\left(2 E_{B}^{2}-E_{f}^{2}\right) \hat{n}+E_{B}^{2}-E_{f}^{2} & -E_{f} E_{B} \\ E_{f} E_{B} & E_{B}^{2}\left(a^{2}+a^{\dagger^{2}}\right)+\left(2 E_{B}^{2}-E_{f}^{2}\right) \hat{n}+E_{B}^{2}\end{array}\right)$,

or in a more compact form as

$$
\begin{aligned}
\bar{H} \tilde{H} \equiv & \left(\left(2 E_{B}^{2}-E_{f}^{2}\right) \hat{n}+E_{B}^{2}\left(a a+a^{\dagger} a^{\dagger}\right)\right) \mathbb{1} \\
& +\left(\begin{array}{cc}
E_{B}^{2}-E_{f}^{2} & -E_{f} E_{B} \\
E_{f} E_{B} & E_{B}^{2}
\end{array}\right) .
\end{aligned}
$$

One can write the linear combination of $\tilde{\mathcal{H}}+\overline{\mathcal{H}}$ and $\overline{\mathcal{H}} \tilde{\mathcal{H}}$ as sum of an operator $\hat{\boldsymbol{J}}$ and a two by two real matrix $\boldsymbol{K}$ as

$$
\mu(\overline{\mathrm{H}}+\tilde{\mathrm{H}})+v \overline{\mathrm{H}} \tilde{\mathrm{H}}=\hat{\boldsymbol{J}} \mathbb{1}+\boldsymbol{K} .
$$

By applying the left hand side of the Eq. (188) on $|\psi\rangle$ we have

$$
(\mu(\overline{\mathrm{H}}+\tilde{\mathrm{H}})+v \overline{\mathrm{H}} \tilde{\mathrm{H}})|\psi\rangle=\left(\mu \epsilon_{0}+\mu \overline{\mathrm{H}}+v \epsilon_{0} \overline{\mathrm{H}}\right)|\psi\rangle .
$$

By choosing $\mu=\epsilon_{0}$ and $v=-1$ we can reduce the problem to

$$
(\hat{\boldsymbol{J}} \mathbb{1}+\boldsymbol{K})|\psi\rangle=\epsilon_{0}^{2}|\psi\rangle
$$

in which $\hat{\boldsymbol{J}}$ and $\boldsymbol{K}$ are

$$
\hat{\boldsymbol{J}}=2 \epsilon_{0} E_{B}\left(a+a^{\dagger}\right)-E_{B}^{2}\left(a a+a^{\dagger} a^{\dagger}\right)-\left(2 E_{B}^{2}-E_{f}^{2}\right) \hat{n},
$$




$$
\boldsymbol{K} \doteq\left(\begin{array}{cc}
E_{f}^{2}-E_{B}^{2} & E_{f} E_{B} \\
-E_{f} E_{B} & -E_{B}^{2}
\end{array}\right) .
$$

Solving Eq. 190 is indeed much easier than our initial problem i.e, $\tilde{\mathcal{H}}|\psi\rangle=$ $\epsilon_{0}|\psi\rangle$. The only thing that we should be careful about is when our new eigenvalue problem has extra double degeneracy in which should be dealt with carefully.

The eigenvector $|\psi\rangle$ in Eq. 190 can be written as $|\psi\rangle=\chi^{R}|\phi\rangle$, where $|\phi\rangle$ is the eigenvalue of $\hat{\boldsymbol{J}}$ and $\chi^{R}$ is the eigenfunction of the following eigenvalue problem

$$
\boldsymbol{K} \chi^{\mathrm{R}}=\lambda \chi^{\mathrm{R}}
$$

and eigenvalues

$$
\lambda_{ \pm}=-E_{B}^{2}+\frac{1}{2} E_{f}^{2} \pm \frac{1}{2} E_{f} \sqrt{E_{f}^{2}-4 E_{B}^{2}}
$$

and eigenvectors

$$
\chi_{ \pm}^{R}=\sqrt{\left|\frac{E_{B}}{E_{f}}\right|}\left[\begin{array}{c}
-\sqrt{C_{ \pm}} \\
1 / \sqrt{C_{ \pm}}
\end{array}\right]
$$

and

$$
\mathrm{C}_{ \pm}=\mathrm{E}_{\mathrm{f}} /\left|\mathrm{E}_{\mathrm{B}}\right| \pm \sqrt{\mathrm{E}_{\mathrm{f}}^{2} / \mathrm{E}_{\mathrm{B}}^{2}-4}
$$

In order to have real eigenvalues in Eq. 194, relation $E_{f}^{2} \geqslant 4 E_{B}^{2}$ must always be satisfied. Solving the eigenvalue problem Eq. 193 leads to

$$
\hat{\boldsymbol{J}}|\phi\rangle=\left(\epsilon_{0}^{2}-\lambda_{ \pm}\right)|\phi\rangle
$$

To solve this eigenvalue problem, we write $\hat{\boldsymbol{J}}$ as a sum of two Hamiltonians $\hat{\boldsymbol{J}}=\hat{\mathrm{H}}_{1}+\hat{\mathrm{H}}_{2}$ where

$$
\begin{aligned}
& \hat{H}_{1}=\left(E_{f}^{2}-2 E_{B}^{2}\right) \hat{n}-E_{B}^{2}\left(a a+a^{\dagger} a^{\dagger}\right), \\
& \hat{H}_{2}=2 \epsilon_{0} E_{B}\left(a+a^{\dagger}\right) .
\end{aligned}
$$


By using canonical transformations

$$
\begin{aligned}
& a=\cosh U \gamma-\sinh U \gamma^{\dagger} \\
& a^{\dagger}=\cosh U \gamma^{\dagger}-\sinh U \gamma,
\end{aligned}
$$

we can write

$$
\begin{aligned}
\hat{\mathrm{H}}_{1}= & 2 \mathrm{E}_{\mathrm{B}}^{2} \sinh \mathrm{U} \cosh \mathrm{U}+\left(\mathrm{E}_{\mathrm{f}}^{2}-2 \mathrm{E}_{\mathrm{B}}^{2}\right) \sinh ^{2} \mathrm{U} \\
& +\left(\gamma \gamma+\gamma^{\dagger} \gamma^{\dagger}\right)\left\{-\mathrm{E}_{\mathrm{B}}^{2}\left(\cosh ^{2} \mathrm{U}+\sinh ^{2} \mathrm{U}\right)\right. \\
& \left.-\left(\mathrm{E}_{\mathrm{f}}^{2}-2 \mathrm{E}_{\mathrm{B}}^{2}\right) \sinh \mathrm{U} \cosh \mathrm{U}\right\} \\
& +\gamma \gamma^{\dagger}\left\{-\left(\mathrm{E}_{\mathrm{f}}^{2}-2 \mathrm{E}_{\mathrm{B}}^{2}\right)\left(\cosh ^{2} \mathrm{U}+\sinh ^{2} \mathrm{U}\right)\right. \\
& \left.+4 \mathrm{E}_{\mathrm{B}}^{2} \sinh \mathrm{U} \cosh \mathrm{U}\right\} .
\end{aligned}
$$

In order to diagonalize $\hat{\mathrm{H}}_{1}$, the coefficient multiplying the $\left(\gamma \gamma+\gamma^{\dagger} \gamma^{\dagger}\right)$ term must be zero, which leads to

$$
\begin{aligned}
\tanh (2 \mathrm{U}) & =\frac{-2 \mathrm{E}_{\mathrm{B}}^{2}}{\mathrm{E}_{\mathrm{f}}^{2}-2 \mathrm{E}_{\mathrm{B}}^{2}}, \\
\sinh ^{2} \mathrm{U} & =-\frac{1}{2}\left[1-\frac{\mathrm{E}_{\mathrm{f}}^{2}-2 \mathrm{E}_{\mathrm{B}}^{2}}{\omega}\right], \\
\cosh ^{2} \mathrm{U} & =\frac{1}{2}\left[1+\frac{\mathrm{E}_{\mathrm{f}}^{2}-2 \mathrm{E}_{\mathrm{B}}^{2}}{\omega}\right], \\
\sinh \mathrm{U} \cosh \mathrm{U} & =-\frac{\mathrm{E}_{\mathrm{B}}^{2}}{\omega},
\end{aligned}
$$

where $\omega=\sqrt{E_{f}^{4}-4 E_{f}^{2} E_{B}^{2}}$. By using the results for sinh $U$ and $\cosh U$ one can write

$$
\begin{aligned}
\hat{\mathrm{H}}_{1}= & \frac{1}{2}\left[\omega-\left(\mathrm{E}_{\mathrm{f}}^{2}-2 \mathrm{E}_{\mathrm{B}}^{2}\right)\right]+\omega \gamma^{\dagger} \gamma \\
& \equiv \mathrm{C}_{1}+\omega \gamma^{\dagger} \gamma,
\end{aligned}
$$


where $C_{1}$ is

$$
\mathrm{C}_{1}=\frac{1}{2}\left[\omega-\left(\mathrm{E}_{\mathrm{f}}^{2}-2 \mathrm{E}_{\mathrm{B}}^{2}\right)\right]
$$

and

$$
\begin{aligned}
\hat{\mathrm{H}}_{2}= & 2 \epsilon_{0} \mathrm{E}_{\mathrm{B}}\left(\mathrm{a}+\mathrm{a}^{\dagger}\right)=2 \epsilon_{0} \mathrm{E}_{\mathrm{B}}(\cosh \mathrm{U}-\sinh \mathrm{U})\left(\gamma+\gamma^{\dagger}\right) \\
& \equiv \mathrm{C}_{2}\left(\gamma+\gamma^{\dagger}\right),
\end{aligned}
$$

where $C_{2}$ is

$$
\mathrm{C}_{2}=2 \epsilon_{0} \mathrm{E}_{\mathrm{B}}(\cosh \mathrm{U}-\sinh \mathrm{U})
$$

The last step to diagonalize $\hat{\mathrm{H}}_{1}+\hat{\mathrm{H}}_{2}$ can be accomplished by using the transformation $\gamma^{\dagger}=\beta^{\dagger}-C_{2} / \omega$ yields

$$
\hat{\mathrm{H}}_{1}+\hat{\mathrm{H}}_{2}=\mathrm{C}_{1}-\frac{\mathrm{C}_{2}^{2}}{\omega}+\omega \beta^{\dagger} \beta
$$

where the eigenvalues of this Hamiltonian are given by

$$
\omega_{n}=\frac{1}{2}\left[\omega-\left(E_{f}^{2}-2 E_{B}^{2}\right)\right]-\frac{4 \epsilon_{0}^{2} E_{B}^{2} E_{f}^{2}}{\omega^{2}}+\omega n .
$$

So the eigenvalues of Eq. 190 are given by $\epsilon_{0}^{2}-\lambda_{ \pm}=\omega_{n}$, leading to

$$
\epsilon_{0}^{2}=\frac{\omega^{3}}{E_{f}^{4}}\left(n+\frac{1}{2}\right) .
$$

In summary the LLs! of graphene in a transverse electric field are given by

$$
\epsilon(n)=-e E l_{B}^{2} k-\operatorname{sgn}(n) \frac{\left(E_{F}^{2}-4 E_{B}^{2}\right)^{3} / 4}{E_{F}^{1 / 2}} \sqrt{|n|},
$$

with eigenvectors

$$
\begin{gathered}
|\psi\rangle_{n}=\sqrt{\left|\frac{E_{B}}{E_{f}}\right|}\left[\begin{array}{c}
-|n-1 ; \beta\rangle \sqrt{C_{+}}-\operatorname{sgn}(n)|n ; \beta\rangle \sqrt{C_{-}} \\
|n-1 ; \beta\rangle / \sqrt{C_{+}}+\operatorname{sgn}(n)|n ; \beta\rangle / \sqrt{C_{-}}
\end{array}\right] \text {for } n \neq 0, \\
|\psi\rangle_{0}=\sqrt{\left|\frac{E_{B}}{E_{f}}\right|}\left[\begin{array}{c}
-\sqrt{C_{-}} \\
1 / \sqrt{C_{-}}
\end{array}\right]|0 ; \beta\rangle \quad \text { for } n=0
\end{gathered}
$$




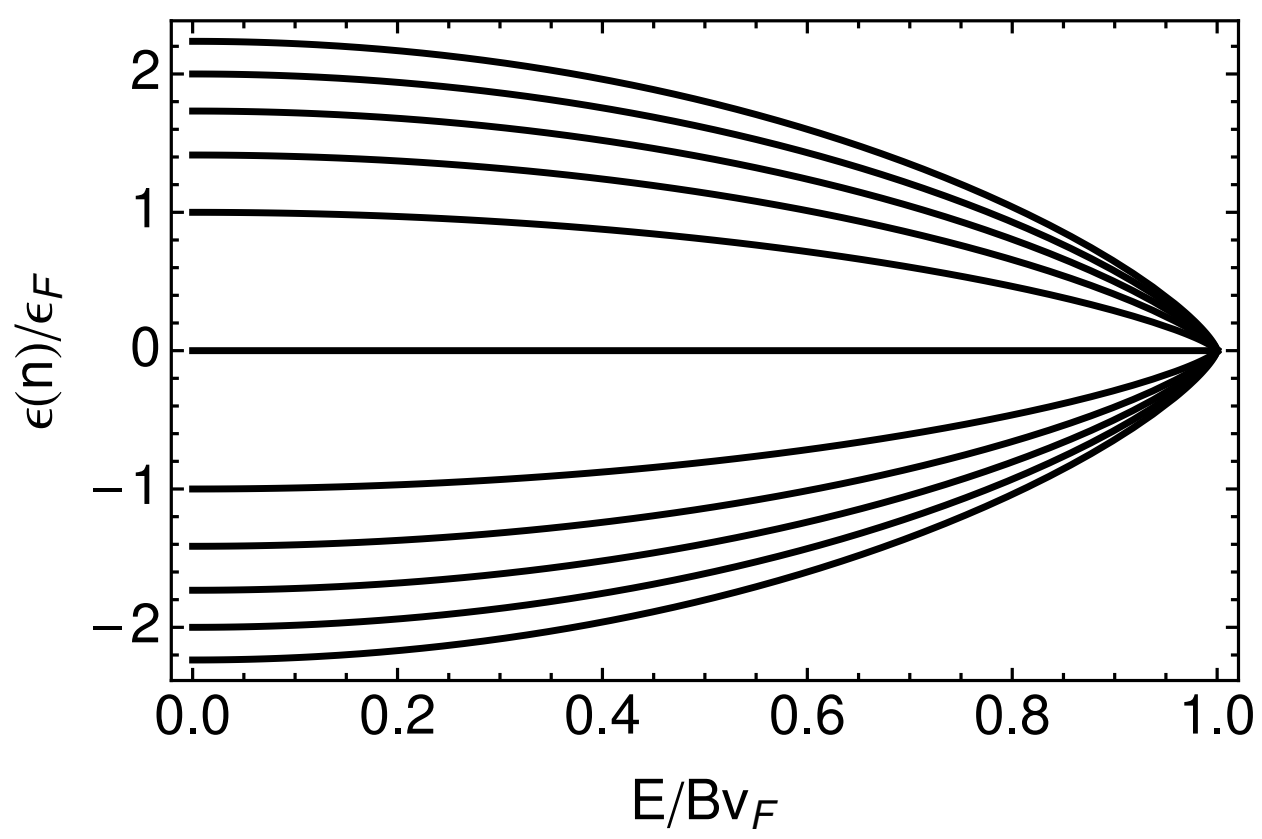

Figure 31: Landau levels in graphene as a function of $E / B v_{F}$ where $E$ is the transverse electric field. Here the term $-e \mathrm{El}_{\mathrm{B}}^{2} \mathrm{k}$ has been chosen to be zero.

In Fig. 31 the first five Landau levels in a monolayer graphene have been shown as a function of $E / B v_{F}$. The condition $E_{F}^{2} \geqslant 4 E_{B}^{2}$ for having real energy eigenvalues implies that $E<B v_{f}$ must always be fulfilled. When $E$ tends towards $B v_{F}$ the Landau levels become closer and closer, and they eventually collapse when $E=$ $\mathrm{B} v_{\mathrm{F}}$. The collapse of Landau levels in the presence of transverse electric field is a novel effect unlike the usual 2 DEG case. We mention that the method used by Peres and Castro (2007) is not valid when $E>B v_{F}$.

\subsubsection{Orbital Rashba Effect of on Landau levels}

As we showed in the previous section the effect of a transverse electric field on the quantized Landau levels shows the collapse of Landau levels when the value of electric to magnetic field ratio reaches the Fermi velocity (Peres and Castro, 2007). However, Lukose et al. (2007) showed by the use of full tight-binding calculations, that this collapse occurs at ratio values smaller than the Fermi velocity. 
In this section, we investigate how orbital Rashba can affect the collapse of Landau levels. Now that we know the exact energy eigenvalues and eigenvectors of a monolayer graphene in a crossed magnetic and electric field, we can study the effect of the orbital Rashba term $\Delta_{R}\left(a+a^{\dagger}\right) \sigma_{z}$ in Eq. 180 by using the perturbation theory.

The general formula for finding energy eigenstates using perturbation theory is given by

$$
\epsilon(n)=\epsilon^{0}(n)+\Delta_{n},
$$

where $\epsilon^{0}(n)$ are the energy eigenvalues of the non-perturbed Hamiltonian, i.e. $H=E_{f}\left(a^{\dagger} \sigma_{-}+a \sigma_{+}\right)+E_{B}\left(a+a^{\dagger}\right) \mathbb{1}-e E l_{B}^{2} k \mathbb{1}$, and $\Delta_{n}$ is the shift in energy due to the perturbation $\Delta_{R}\left(a+a^{\dagger}\right) \sigma_{z}$ given by

$$
\Delta_{n}=\lambda V_{n n}+\lambda^{2} \sum_{k \neq m} \frac{\left|V_{n m}\right|^{2}}{E_{n}^{0}-E_{m}^{0}}+\ldots
$$

where

$$
V_{n m} \equiv\left\langle n^{0}|V| m^{0}\right\rangle
$$

with $\left|n^{0}\right\rangle$ being the eigenvectors of the unperturbed Hamiltonian (Sakurai, 1994). Applying the energy eigenvalues and eigenvectors of the unperturbed Hamiltonian given by Eq. 210 and Eq. 211 yields to

$$
\begin{aligned}
\epsilon(n) / E_{F}= & \epsilon^{0}(n) / E_{F}+\bar{V}_{O R}^{2} \frac{\alpha^{2}\left(1-\alpha^{2}\right)^{3 / 4}}{\operatorname{sgn}(m) \sqrt{|m|}-\operatorname{sgn}(n) \sqrt{|n|}} \sum_{\substack{m \neq n \\
m \neq 0}}\left[\alpha^{2}\left(1-\alpha^{2}\right) \delta_{n-1, m-1}\right. \\
& +\frac{1}{4}(|n|+|m|-2 \operatorname{sgn}(n) \operatorname{sgn}(m) \sqrt{1+|n|} \sqrt{1+|m|})\left(\delta_{n-1, m}+\right. \\
& \left.\left.\delta_{n-1, m-2}+\delta_{n-1,-m-2}+\delta_{n-1,-m}\right)\right]
\end{aligned}
$$

where $\alpha \equiv \mathrm{E} / \mathrm{B} v_{\mathrm{F}}$ and $\overline{\mathrm{V}}_{\mathrm{OR}}$ is given by

$$
\Delta_{\mathrm{R}} / \mathrm{E}_{\mathrm{F}}=\frac{\mathrm{V}_{\mathrm{OR}}}{e l_{\mathrm{B}}} \frac{\alpha}{2}=\overline{\mathrm{V}}_{\mathrm{OR}} \frac{\alpha}{2}
$$

There are several points worth mentioning from Eq. 215. First of all, our calculations show that the orbital Rashba term does not affect the Landau levels in 


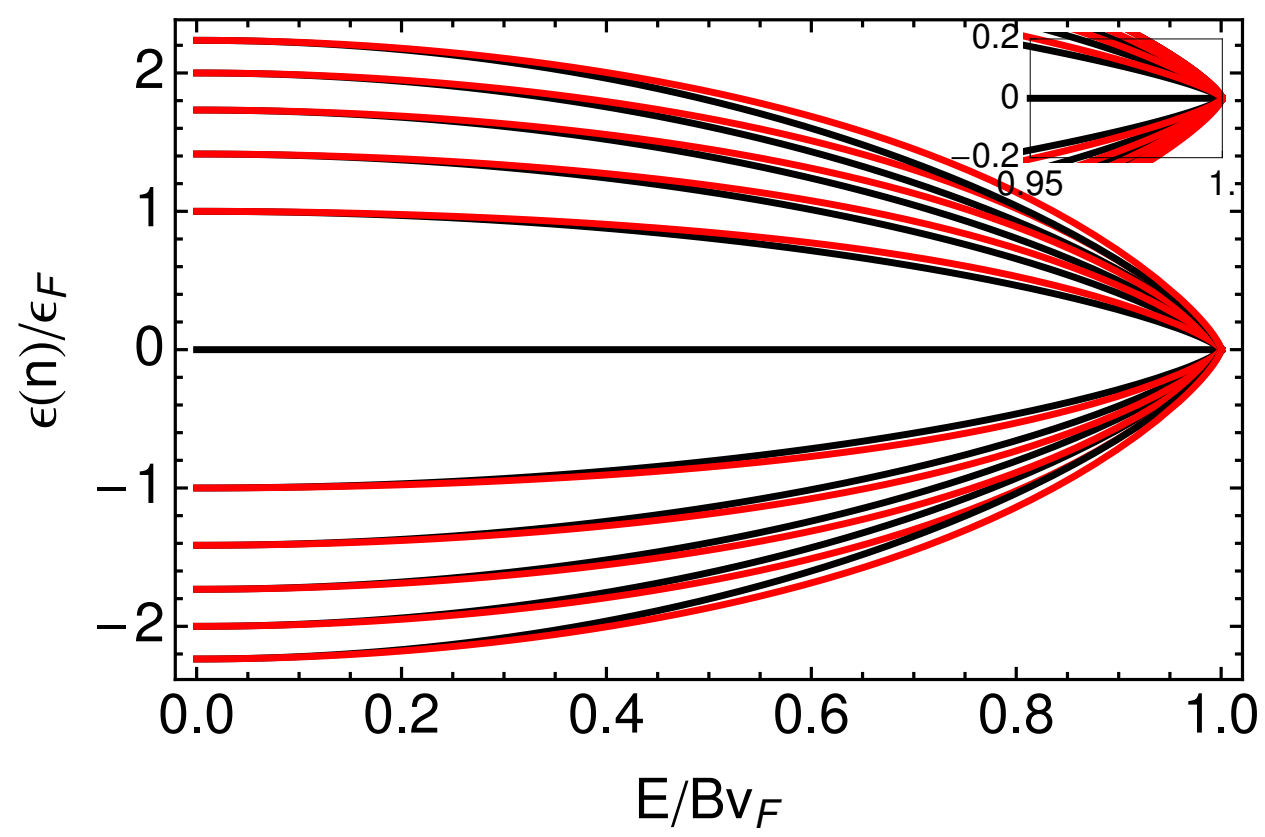

Figure 32: Landau levels in graphene as a function of $E / B v_{F}$ where $E$ is the transverse electric field. Energy levels with red (black) colour correspond to $\bar{V}_{\mathrm{OR}}=0.2$ $\left(\overline{\mathrm{V}}_{\mathrm{OR}}=0\right)$.

first order. However, second-order perturbation is non-zero and the correction to energy levels are proportional to the term $\alpha^{2}\left(1-\alpha^{2}\right)^{\gamma}$. This implies that when $\alpha=0$ (no electric field applied), the correction terms vanish, and the Landau levels are the same as for the case of the unperturbed case. The same story is true $\mathrm{E}=\mathrm{B} v_{\mathrm{F}}$ which implies that $\alpha=1$ and again the correction term disappears. However, when $0<\alpha<1$ the correction terms are not zero. Our calculations also show that the Landau level with $n=0$ is not affected even by the second order perturbation approximation. Fig. 32 shows how the orbital Rashba can affect the Landau levels.

\subsection{AC TRANSPORT OF MONOLAYER GRAPHENE IN THE PRESENCE OF AN ELECTRIC FIELD}

As we discussed in Sec. 1.7, there has been a confusion in theoretical results found for minimal conductivity of graphene systems. Firstly, the experimental value ob- 
tained for the universal minimal conductivity is almost three times larger than the results obtained by theoretical studies. Secondly, there have been different theoretical results for the minimal conductivity of graphene (Ziegler, 2007). For example by using the Kubo formula, it has been shown that for a clean graphene system, at zero temperature and finite frequency, the minimal conductivity calculated per spin per valley is given by $\sigma_{\min }=e^{2} / 8 \mathrm{~h}$ and is independent of the frequency (Ludwig et al., 1994; Cserti, 2007). However Bernád et al. (2010) showed that by taking trigonal warping into account $\sigma_{\min }$ has a larger value than $e^{2} / 8 \mathrm{~h}$.

Here in this section we study the effect of the orbital Rashba term on the electronic transport of a monolayer graphene. We apply standard linear response or Kubo formalism in order to find how the AC electric transport in monolayer graphene is affected by this term. In order to calculate the AC conductivity of our system, we divide the Hamiltonian into two parts, the free Hamiltonian $\mathrm{H}_{0}$ and the perturbation due to a time-dependent external uniform electric field $\delta \mathrm{H}$,

$$
\begin{aligned}
& H=H_{0}+\delta H, \\
& H_{0}=\hbar v\left(\sigma_{x} k_{x}+\sigma_{y} k_{y}\right), \\
& \delta H=\lim _{\alpha \rightarrow \infty}\left(e \vec{E} \cdot \vec{r}+V_{O R}(\vec{k} \times \vec{E}) \cdot \hat{z} \sigma_{z}\right) e^{-i \omega t+\alpha t} .
\end{aligned}
$$

For finding the linear response of the system, we start by calculating the time evolution of the density matrix $\rho=\rho_{0}+\delta \rho$ where $\rho_{0}$ is the density matrix of the free Hamiltonian $\mathrm{H}_{0}$ and $\delta \rho$ is the perturbation (Madelung, 1978). The time evolution of density matrix can be obtained from iћ $\dot{\rho}=[H, \rho]$ which leads to

$$
i \hbar \dot{\delta} \rho=\left[H_{0}, \delta \rho\right]+\left[\delta H, \rho_{0}\right]
$$

We have neglected terms of the order $\delta \rho \delta \mathrm{H}$ on the right-hand side of the equation. It is more convenient to work in the interaction picture where

$$
\delta \rho=e^{-\frac{i}{\hbar} H_{0} t} \Delta \rho e^{\frac{i}{\hbar} H_{0} t} .
$$

Replacing relation 219 in Eq. 218 yields

$$
i \hbar \Delta \rho=e^{\frac{i}{\hbar} H_{0} t}\left[\delta H, \rho_{0}\right] e^{-\frac{i}{\hbar} H_{0} t}
$$


We assume that the field is applied at time $t=-\infty$ and increases adiabatically to its value at time $t=0$, so

$$
\delta \rho(t=0)=\Delta \rho(t=0)=\frac{1}{i \hbar} \int_{-\infty}^{0} d t e^{\frac{i}{\hbar} H_{0} t}\left[\delta H, \rho_{0}\right] e^{-\frac{i}{\hbar} H_{0} t} .
$$

For finding the response of the system to the electric field, we calculate the expectation value of current operator

$$
\begin{aligned}
\langle j\rangle= & \operatorname{Trace}(j \delta \rho) \\
& =\frac{1}{i \hbar} \int_{-\infty}^{0} \operatorname{dtTrace}\left(j e^{\frac{i}{\hbar} H_{0} t}\left[\delta H, \rho_{0}\right] e^{-\frac{i}{\hbar} H_{0} t}\right) .
\end{aligned}
$$

By replacing $\delta \mathrm{H}$ we can write

$$
\begin{aligned}
j_{\mu}= & \frac{1}{i \hbar} \lim _{\alpha \rightarrow \infty} \int_{-\infty}^{0} d t e^{-i \omega t+\alpha t} \\
& \times \operatorname{Trace}\left(j _ { \mu } e ^ { \frac { i } { \hbar } H _ { 0 } t } \left(\left[e E_{\mu} r_{\mu}+e E_{v} r_{v}, \rho_{0}\right]\right.\right. \\
& \left.\left.+V_{O R}\left(k_{\mu} E_{v}-k_{v} E_{\mu}\right)\left[\sigma_{z}, \rho_{0}\right]\right) e^{-\frac{i}{\hbar} H_{0} t}\right) .
\end{aligned}
$$

and the conductivity is

$$
\begin{aligned}
\sigma_{\mu \mu}= & \frac{1}{i \hbar} \lim _{\alpha \rightarrow \infty} \int_{-\infty}^{0} d t e^{-i \omega t+\alpha t} \operatorname{Trace}\left(j _ { \mu } e ^ { \frac { i } { \hbar } H _ { 0 } t } \left(\left[\operatorname{er}_{\mu}, \rho_{0}\right]\right.\right. \\
& \left.\left.+\alpha_{\mu} V_{O R} k_{v}\left[\sigma_{z}, \rho_{0}\right]\right) e^{-\frac{i}{\hbar} H_{0} t}\right), \\
\sigma_{\mu \nu}= & \frac{1}{i \hbar} \lim _{\alpha \rightarrow \infty} \int_{-\infty}^{0} d t e^{-i \omega t+\alpha t} \operatorname{Trace}\left(j _ { \mu } e ^ { \frac { i } { \hbar } H _ { 0 } t } \left(\left[\operatorname{er}_{\nu}, \rho_{0}\right]\right.\right. \\
& \left.\left.+\alpha_{\mu \nu} V_{O R} k_{\mu}\left[\sigma_{z}, \rho_{0}\right]\right) e^{-\frac{i}{\hbar} H_{0} t}\right)
\end{aligned}
$$

where $\alpha_{\mu}$ and $\alpha_{\mu v}$ are defined as

$$
\alpha_{\mu \nu}=\left\{\begin{array}{ll}
-1 & \text { if } \mu \equiv x, v \equiv y, \\
+1 & \text { if } \mu \equiv y, v \equiv x .
\end{array} \quad \alpha_{\mu \mu} \equiv \alpha_{\mu}= \begin{cases}-1 & \text { if } \mu \equiv x \\
+1 & \text { if } \mu \equiv y\end{cases}\right.
$$

First we focus on calculating $\sigma_{\mu \nu}$

$$
\sigma_{\mu \nu}=\lim _{\alpha \rightarrow 0^{+}} \int_{-\infty}^{0} d t e^{-i \omega t+\alpha t} K_{\mu \nu}
$$


with the Kernel

$$
K_{\mu v}=\frac{1}{i \hbar} \operatorname{Trace}\left(j_{\mu} e^{\frac{i}{\hbar} H_{0} t}\left(\left[e r_{v}, \rho_{0}\right]+\alpha_{\mu \nu} V_{O R} k_{\mu}\left[\sigma_{z}, \rho_{0}\right]\right) e^{-\frac{i}{\hbar} H_{0} t}\right)
$$

By using the following relations

$$
\begin{aligned}
& {\left[r_{\mu}, \rho_{0}\right]=\rho_{0} \int_{0}^{1 / K_{\beta} T} d \lambda e^{\lambda H_{0}}\left[H_{0}, r_{\mu}\right] e^{-\lambda H_{0},}} \\
& {\left[\sigma_{z}, \rho_{0}\right]=\rho_{0} \int_{0}^{1 / K_{\beta} T} d \lambda e^{\lambda H_{0}}\left[H_{0}, \sigma_{z}\right] e^{-\lambda H_{0},},}
\end{aligned}
$$

the kernel will be divided into two parts

$$
K_{\mu \nu}=K_{\mu \nu}^{I}+K_{\mu \nu}^{I I}
$$

where $\mathrm{K}_{\mu \nu}^{\mathrm{I}}$ is the kernel of the conductivity of monolayer graphene without considering the orbital Rashba effect, which has been calculated in Chap. 1 and also in the work of (Bernád et al., 2010):

$$
K_{\mu \nu}^{I}=\frac{1}{i \hbar} \operatorname{Trace}\left(j_{\mu} \rho_{0} \int_{0}^{\frac{1}{K_{\beta} T}} d \lambda e^{\frac{i}{\hbar} H_{0}(t-i \hbar \lambda)} e\left[H_{0}, r_{\nu}\right] e^{-\frac{i}{\hbar} H_{0}(t-i \hbar \lambda)}\right) \text {. }
$$

$\mathrm{K}_{\mu \nu}^{\mathrm{II}}$ is the kernel of the correction to the conductivity due to orbital-Rashba term

$$
K_{\mu \nu}^{I I}=\frac{1}{i \hbar} \operatorname{Trace}\left(j_{\mu} \rho_{0} \int_{0}^{\frac{1}{K_{\beta} T}} d \lambda e^{\frac{i}{\hbar} H_{0}(t-i \hbar \lambda)} \alpha_{\mu \nu} V_{O R} k_{\mu}\left[H_{0}, \sigma_{z}\right] e^{-\frac{i}{\hbar} H_{0}(t-i \hbar \lambda)}\right) \text {. }
$$

By using the eigenstates of the free Hamiltonian $\mathrm{H}_{0}$ to calculate the trace and also replacing the current operator by its commutation relation $j_{\mu}=\frac{-i e}{\hbar}\left[\mathrm{H}_{0}, r_{\mu}\right]$ we find

$$
\begin{aligned}
K_{\mu \nu}^{\mathrm{II}}= & \frac{-e \alpha_{\mu \nu} V_{\mathrm{OR}}}{\hbar^{2}} \sum_{n, m} k_{\mu} e^{\frac{i}{\hbar}\left(\epsilon_{m}-\epsilon_{n}\right) t} \frac{e^{\frac{\left(\epsilon_{m}-\epsilon_{n}\right)}{k_{\beta} T}}-1}{\epsilon_{\mathrm{m}}-\epsilon_{n}} \\
& \times\left\langle\epsilon_{\mathrm{n}}\left|\left[\mathrm{H}_{0}, r_{\mu}\right] \rho_{0}\right| \epsilon_{m}\right\rangle\left\langle\epsilon_{m}\left|\left[\mathrm{H}_{0}, \sigma_{z}\right]\right| \epsilon_{n}\right\rangle .
\end{aligned}
$$

By applying the definition of the density matrix $\rho_{0}$ as

$$
\begin{aligned}
& \qquad \rho_{0}=\mathrm{Z}_{0}^{-1} \exp \left(\frac{-\mathrm{H}_{0}}{\mathrm{~K}_{\beta} \mathrm{T}}\right) \\
& \text { where } \quad \mathrm{Z}_{0}=\operatorname{Trace}\left(\exp \left(\frac{-\mathrm{H}_{0}}{\mathrm{~K}_{\beta} \mathrm{T}}\right)\right),
\end{aligned}
$$


$\mathrm{K}_{\mu \nu}^{\mathrm{II}}$ has the following form

$$
\begin{aligned}
K_{\mu \nu}^{I I}= & \frac{-e \alpha_{\mu v} V_{O R}}{\hbar^{2}} \sum_{n, m} k_{\mu} e^{\frac{i}{\hbar}\left(\epsilon_{m}-\epsilon_{n}\right) t} \frac{f\left(\epsilon_{n}\right)-f\left(\epsilon_{m}\right)}{\epsilon_{m}-\epsilon_{n}} \\
& \times\left\langle\epsilon_{n}\left|\left[H_{0}, r_{\mu}\right]\right| \epsilon_{m}\right\rangle\left\langle\epsilon_{m}\left|\left[H_{0}, \sigma_{z}\right]\right| \epsilon_{n}\right\rangle,
\end{aligned}
$$

in which we have used the relation $\rho_{0}\left|\epsilon_{n}\right\rangle=f\left(\epsilon_{n}\right)\left|\epsilon_{n}\right\rangle$ where $f(\epsilon)$ denotes the Fermi function. Consequently $\sigma_{\mu \mu}^{\mathrm{II}}$ reads as

$$
\begin{aligned}
\sigma_{\mu \nu}^{I I}= & \frac{i e \alpha_{\mu \nu} V_{O R}}{\hbar} \lim _{\alpha \rightarrow 0^{+}} \sum_{n, m} k_{\mu} \frac{\left\langle\epsilon_{n}\left|\left[H_{0}, r_{\mu}\right]\right| \epsilon_{m}\right\rangle\left\langle\epsilon_{m}\left|\left[H_{0}, \sigma_{z}\right]\right| \epsilon_{n}\right\rangle}{\left(\epsilon_{m}-\epsilon_{n}-\hbar \omega-i \alpha\right)} \\
& \times \frac{f\left(\epsilon_{n}\right)-f\left(\epsilon_{m}\right)}{\epsilon_{m}-\epsilon_{n}} .
\end{aligned}
$$

By using continuum descriptions of the band structure, the eigenstates of the clean graphene Hamiltonian $\mathrm{H}_{0}$ can be written as a product of $|k\rangle$ wave-vector in real space and a spinor $|s\rangle_{k}$

$$
\left|\epsilon_{\mathrm{n}}\right\rangle=|\mathrm{k}\rangle \otimes|\mathrm{s}\rangle_{\mathrm{k}}
$$

For the Hamiltonian of graphene $\mathrm{H}_{0}$ the energy eigenstates and eigenvalues are given by

$$
\begin{array}{r}
\epsilon_{\mathrm{ks}}=s \hbar v_{\mathrm{f}}|\mathrm{k}|, \\
|s\rangle=\frac{1}{\sqrt{2}}\left(\begin{array}{c}
1 \\
s e^{i \theta}
\end{array}\right),
\end{array}
$$

where $s= \pm$ distinguishes the electron and hole bands. The commutation relations $\left[\mathrm{H}_{0}, \mathrm{r}_{\mu}\right]$ and $\left[\mathrm{H}_{0}, \sigma_{z}\right]$ are diagonal in $|k\rangle$ space so

$$
\begin{aligned}
& {\left[\mathrm{H}_{0}, \mathrm{r}_{\mu}\right]|k\rangle=\mathrm{W}_{\mu}(\mathrm{k})|\mathrm{k}\rangle,} \\
& {\left[\mathrm{H}_{0}, \sigma_{z}\right]|k\rangle=\mathrm{V}(\mathrm{k})|\mathrm{k}\rangle .}
\end{aligned}
$$

Therefore, $\sigma_{\mu \nu}^{\mathrm{II}}$ has the form

$$
\begin{aligned}
\sigma_{\mu \nu}^{\mathrm{II}}= & \frac{i e \alpha_{\mu \nu} V_{\mathrm{OR}}}{\hbar} \lim _{\alpha \rightarrow 0^{+}} \sum_{s, s^{\prime}} \int \frac{d^{2} k}{(2 \pi)^{2}} k_{\mu} \frac{\left\langle s\left|W_{\mu}(k)\right| s^{\prime}\right\rangle_{k}\left\langle s^{\prime}|V(k)| s\right\rangle_{k}}{\left(\epsilon_{k s^{\prime}}-\epsilon_{k s}-\hbar \omega-i \alpha\right)} \\
& \times \frac{f\left(\epsilon_{\mathrm{ks}}\right)-f\left(\epsilon_{\mathrm{ks}}{ }^{\prime}\right)}{\epsilon_{\mathrm{ks}}{ }^{\prime}-\epsilon_{\mathrm{ks}}} .
\end{aligned}
$$


First, we investigate the inter-band $\left(s \neq s^{\prime}\right)$ contribution to the conductivity

$$
\begin{aligned}
\frac{\sigma_{\mu \nu}^{\mathrm{II}}}{\sigma_{0}}= & \frac{-\alpha_{\mu} \mathrm{V}_{\mathrm{OR}}}{e} \frac{1}{2 \hbar \omega} \sum_{s \neq s^{\prime}} \int d^{2} k k_{\mu} \delta\left(\hbar \omega-\left(\epsilon_{\mathrm{ks}}{ }^{\prime}-\epsilon_{\mathrm{ks}}\right)\right) \\
& \times W_{\mu}^{s s^{\prime}}(k) \mathrm{V}^{s^{\prime}}{ }^{\prime}(k) g\left(\frac{\hbar \omega}{2 k_{\beta} T^{\prime}}, \frac{\mu}{k_{\beta} \mathrm{T}}\right),
\end{aligned}
$$

where we have used the relations

$$
\lim _{\alpha \rightarrow 0^{+}} \frac{1}{i} \frac{1}{\epsilon_{\mathrm{ks}^{\prime}}-\epsilon_{\mathrm{ks}}-\hbar \omega-i \hbar \alpha}=\pi \delta\left(\epsilon_{\mathrm{ks}}-\epsilon_{\mathrm{ks}}-\hbar \omega\right),
$$

and

$$
\begin{aligned}
& \mathrm{V}^{s^{\prime}} \mathrm{s}(\mathrm{k})=\left\langle\mathrm{s}^{\prime}|\mathrm{V}(\mathrm{k})| \mathrm{s}\right\rangle, \\
& \mathrm{W}_{\mu}^{s^{\prime}}(\mathrm{k})=\left\langle\mathrm{s}^{\prime}\left|\mathrm{W}_{\mu}(\mathrm{k})\right| \mathrm{s}^{\prime}\right\rangle .
\end{aligned}
$$

Also, we introduced the abbreviation

$$
g(\xi, \eta)=\frac{\sinh \xi}{\cosh \xi+\cosh \eta} .
$$

Performing the summation over $s$ and $s^{\prime}$ leads to

$$
\begin{aligned}
\frac{\sigma_{\mu \nu}^{\mathrm{II}}}{\sigma_{0}}= & \frac{-\alpha_{\mu} V_{\mathrm{OR}}}{e} \frac{g\left(\frac{\hbar \omega}{2 \mathrm{~K} \beta \mathrm{T}}, \frac{\mu}{\mathrm{K}_{\beta} \mathrm{T}}\right)}{2 \hbar \omega} \int \mathrm{d}^{2} \mathrm{k} \mathrm{k}_{\mu}\left(\mathrm{W}_{\mu}^{+-} \mathrm{V}^{-+} \delta\left(\hbar \omega-\left(\epsilon_{\mathrm{k}-}-\epsilon_{\mathrm{k}+}\right)\right)\right. \\
& \left.+W_{\mu}^{-+} \mathrm{V}^{+-} \delta\left(\hbar \omega-\left(\epsilon_{\mathrm{k}+}-\epsilon_{\mathrm{k}-}\right)\right)\right) .
\end{aligned}
$$

It is straight forward to calculate $\mathrm{V}^{s^{\prime}}(\mathrm{k})$ and $\mathrm{W}^{s s^{\prime}}(\mathrm{k})$ for interband transition $\left(s \neq s^{\prime}\right)$ using the eigenstates of Hamiltonian $\mathrm{H}_{0}$ in Eq. 236

$$
\begin{aligned}
& W_{\mu}^{+-}(k)=<+\left|\left[H_{0}, r_{\mu}\right]\right|->=-i \hbar v_{f}<+\left|\sigma_{\mu}\right|->=\frac{\alpha_{\mu} \hbar v_{f} k_{v}}{k}, \\
& \mathrm{~V}^{-+}(\mathrm{k})=<-\left|\left[\mathrm{H}_{0}, \sigma_{z}\right]\right|+>=-2 i \hbar v_{\mathrm{f}}\left(\mathrm{k}_{\mathrm{x}}<-\left|\sigma_{\mathrm{y}}\right|+>-\mathrm{k}_{\mathrm{y}}<-\left|\sigma_{\mathrm{x}}\right|+>\right) \\
& =-2 \hbar v_{f} k \text {. }
\end{aligned}
$$

Thus $\sigma_{\mu \nu}^{\mathrm{II}}$ can be written as

$$
\frac{\sigma_{\mu \nu}^{\mathrm{II}}}{\sigma_{0}}=\frac{\alpha_{\mu} \alpha_{\mu \nu} V_{\mathrm{OR}}}{e} \frac{g\left(\frac{\hbar \omega}{2 \mathrm{~K}_{\beta} \mathrm{T}}, \frac{\mu}{\mathrm{K}_{\beta} \mathrm{T}}\right)}{\hbar \omega} \int d^{2} k \hbar^{2} v_{\mathrm{F}}^{2} k_{\mu} k_{v}\left(\delta\left(\hbar \omega+2 \hbar v_{\mathrm{F}}|\mathrm{k}|\right)+\delta\left(\hbar \omega-2 \hbar v_{\mathrm{F}}|\mathrm{k}|\right)\right) \text {. }
$$


By using relation $k=\frac{v_{f}}{\omega} k$ to make the equation dimensionless, we reach

$$
\frac{\sigma_{\mu \nu}^{\mathrm{II}}}{\sigma_{0}}=\frac{\alpha_{\mu} \alpha_{\mu \nu} V_{\mathrm{OR}}}{e} g\left(\frac{\hbar \omega}{2 \mathrm{~K}_{\beta} \mathrm{T}}, \frac{\mu}{\mathrm{K}_{\beta} \mathrm{T}}\right) \frac{\omega^{2}}{v_{\mathrm{F}}^{2}} \int d^{2} \kappa \kappa_{\mu} \kappa_{\nu}(\delta(1+2|\kappa|)+\delta(1-2|\kappa|)) \Theta(\omega),
$$

where $\Theta$ is the Heaviside step function. Finally, the correction due to orbitalRashba term is given by

$$
\frac{\sigma_{x x}^{\mathrm{II}}}{\sigma_{0}}=\frac{\sigma_{y y}^{\mathrm{II}}}{\sigma_{0}}=\frac{\mathrm{V}_{\mathrm{OR}}}{e} \frac{\omega^{2}}{v_{f}^{2}} \frac{\pi}{16} \mathrm{~g}\left(\frac{\hbar \omega}{2 \mathrm{k}_{\beta} \mathrm{T}}, \frac{\mu}{\mathrm{k}_{\beta} \mathrm{T}}\right) \Theta(\omega) .
$$

By the same calculations, one can find that the orbital-Rashba does not affect off-diagonal elements of the conductivity tensor components $\sigma_{x y}$ or $\sigma_{y x}$. For intraband transitions when $s=s^{\prime}, V^{s s}(k)$ in Eq. 239 becomes zero and so the orbital Rashba term does not change the intraband conductivity. Hence, the conductivity of monolayer graphene in the presence of orbital Rashba is given by

$$
\frac{\sigma_{v v}}{\sigma_{0}}=\frac{\sigma_{v v}^{\mathrm{I}}}{\sigma_{0}}+\frac{\sigma_{\nu v}^{\mathrm{II}}}{\sigma_{0}}=g\left(\frac{\hbar \omega}{2 \mathrm{k}_{\beta} \mathrm{T}}, \frac{\mu}{\mathrm{k}_{\beta} \mathrm{T}}\right) \Theta(\omega) \Gamma\left(\frac{\mathrm{V}_{\mathrm{OR}}}{e} \frac{\omega^{2}}{2 v_{\mathrm{f}}^{2}}\right) \text {. }
$$

where $\Gamma(\zeta)=\frac{\pi}{8}(1+\zeta)$. Note that at the limit of zero temperature $g\left(\frac{\hbar \omega}{2 k_{\beta} T}, \frac{\mu}{k_{\beta} T}\right)=$ 1.

Here we have shown that by taking the orbital Rashba term into account, the minimal conductivity of clean graphene system at zero temperature and for finite $\omega$ in contrast to the results previously found (Ludwig et al., 1994; Cserti, 2007; Bernád et al., 2010) is not independent of frequency.

\subsection{RABI OSCILLATIONS IN THE PRESENCE OF AN IN-PLANE ELECTRIC FIELD}

In quantum optics the Jaynes-Cumming (JC) model is a fully quantum mechanical description of coupling a spinor (two-level system atom) to an external single mode electromagnetic field, and the Hamiltonian describing this feature is given by

$$
\mathrm{H}_{\mathrm{JC}}=\Gamma\left(\mathrm{a}^{\dagger} \sigma_{-}+\sigma_{+} \mathrm{a}\right)+\Delta \sigma_{z}
$$


where $\Gamma$ is the coupling between the two-level atom and electromagnetic field, $\mathrm{a}^{\dagger}(\mathrm{a})$ are the photon creation(annihilation) operators, $\sigma_{+}\left(\sigma_{-}\right)$are raising(lowering) operators of the two-level atom and $\delta$ is called the detuning frequency between the two-level system and the field. According to this Hamiltonian, the absorption of a photon is accompanied by an upward transition $\left(\sigma_{+} a\right)$ while a downward transition in two-level system atom is accompanied by a photon emission $\left(\mathrm{a}^{\dagger} \sigma_{-}\right)$. This interaction leads to a periodic exchange between field and two-level system and is known as Rabi oscillations.

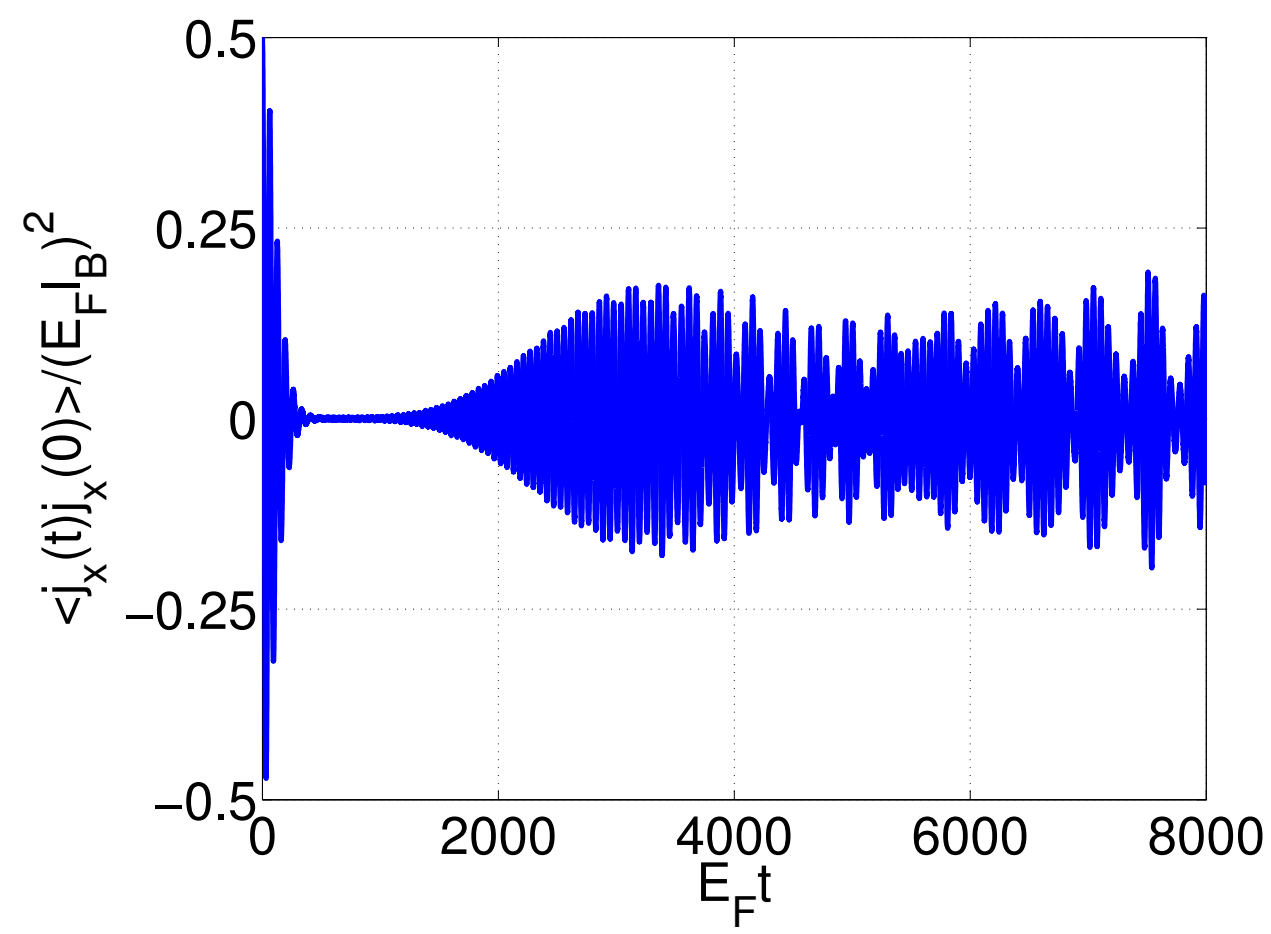

Figure 33: Time evolution of $\left\langle j_{x}(t) j_{x}(0)>\right.$ in the absence of an electric field $\left(E_{B}=\right.$ $\left.0, \Delta_{\mathrm{R}}=0\right)$

In quantum optics one can obtain the spectrum of Rabi oscillations by calculating dynamical correlation function $C_{x x}(t)=\left\langle\sigma_{x}(t) \sigma_{x}(0)\right\rangle$ (symmetric dipoledipole correlator) and $C_{x y}(t)=\left\langle\sigma_{x}(t) \sigma_{y}(0)\right\rangle$ (antisymmetric dipole-dipole correlator) which describe the transitions between states.

The Hamiltonian of graphene in the presence of a perpendicular magnetic field is the same as the well-known Jaynes-Cummings (JC) Hamiltonian in quantum optics. As we discussed in Sec. 1.5.1, Dirac fermions in graphene in the 
presence of a magnetic field are described by $H=v_{f}\left(\tau \pi_{x} \sigma_{x}+\pi_{y} \sigma_{y}\right)+\Delta \sigma_{z}$ where $\vec{\pi}=\vec{p}-e \frac{\vec{A}}{c}$ (minimal coupling), $v_{f}$ is the Fermi velocity and $\tau= \pm 1$ distinguishes the two valleys and $\Delta$ is a mass term. One can define bosonic operators $\mathrm{a}^{\dagger}=\frac{\pi^{\dagger}}{\sqrt{2 \mathrm{eB}}}$ and $a=\frac{\pi}{\sqrt{2 e B}}$ in which $\pi^{ \pm} \equiv \pi_{x} \pm i \pi_{y}$. These operators satisfy the standard commutation relation $\left[a, a^{\dagger}\right]=1$, and by using them, the Hamiltonian of graphene in the presence of a magnetic field can be written as $H=\Omega\left(a^{\dagger} \sigma_{-}+a \sigma_{+}\right)$with $\sigma_{ \pm} \equiv \sigma_{x} \pm i \sigma_{y}$. In this context $\left(\sigma_{+}\left(\sigma_{-}\right)\right)$acting on the pseudospin (sublattices), $\mathrm{a}^{\dagger}(\mathrm{a})$ can be translated to operators action on Landau levels in graphene and $\Delta$ represents a possible gap. To see the Rabi oscillation in graphene first one should prepare quasiparticles to be in a certain Landau level as their initial state. This state is a non-equilibrium state for quasiparticles because it is not an eigenstate of the Hamiltonian of graphene, and quasiparticles will go from this initial state to other Landau levels. The oscillation between Landau levels continues until the system reaches an equilibrium state which will be a superposition of Landau levels. Since in graphene $\sigma_{x(y)}$ play the role of current operators $j_{x(y)}=\mathfrak{i}[\mathrm{H}, x(y)]=v_{F} \sigma_{x(y)}$, the correlation function $C_{x x}(t)=\left\langle\sigma_{x}(t) \sigma_{x}(0)\right\rangle$ and $C_{x y}(t)=\left\langle\sigma_{x}(t) \sigma_{y}(0)\right\rangle$ play the role of the longitudinal and transverse current correlation functions in graphene. Therefore, one can expect to observe Rabi oscillations in the response function of Landau-quantized graphene. Dóra et al. (2009) showed that Rabi oscillations in the optical response of graphene are observable. Since preparing graphene in an arbitrary initial state is not accessible by experiiment, they have considered a thermal ensemble averaging in their calculations. They have calculated longitudinal and transverse current-current correlation functions and observed a collapse and revival in their spectrum as the signature of Rabi oscillations.

The Hamiltonian of graphene in the presence of a perpendicular magnetic field and in-plane electric field is given by Eq. 180. So in the presence of an inplane electric field in a particular direction e.g. $y$ direction, by considering the 


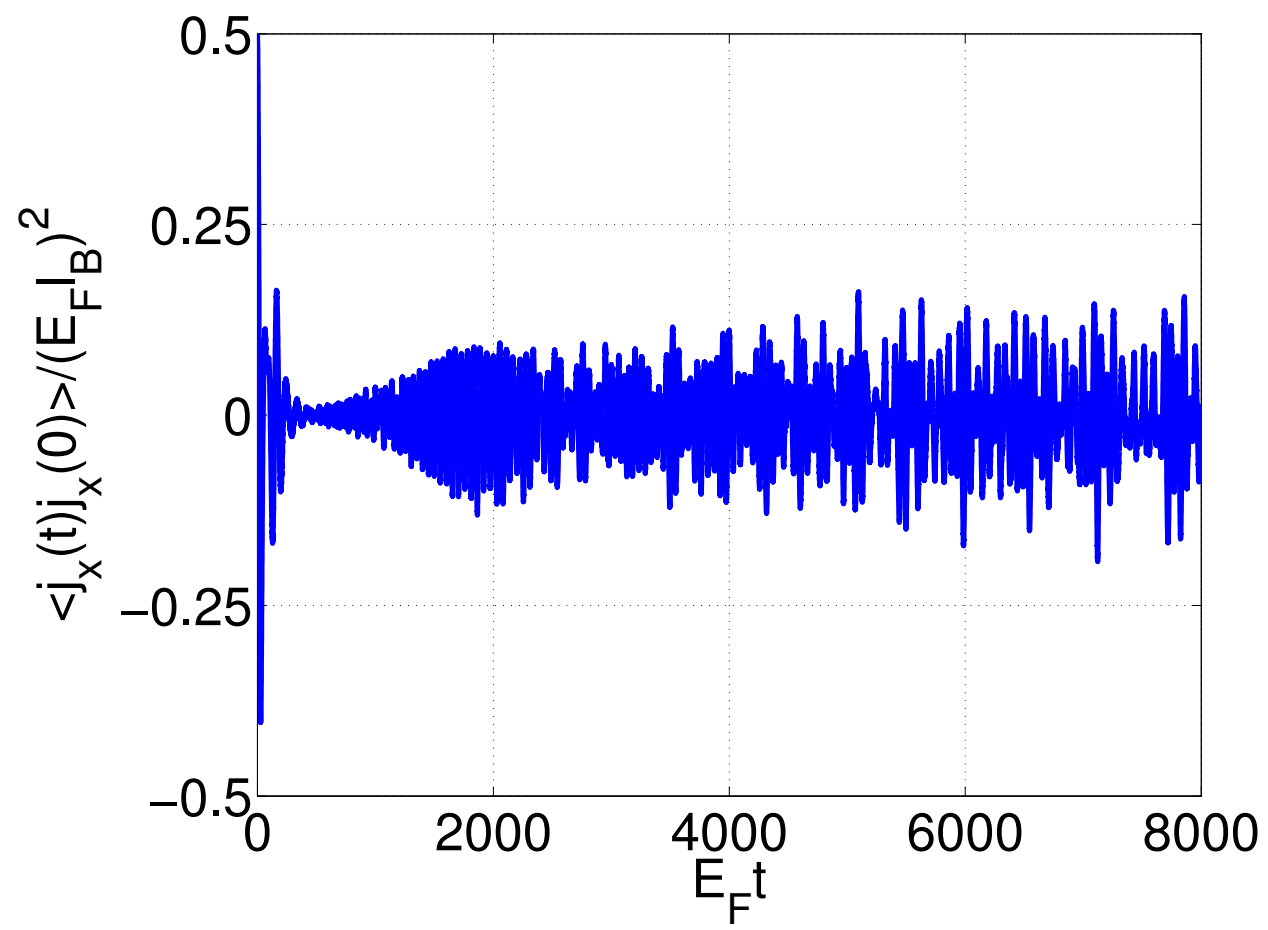

Figure 34: Time evolution of $\left\langle j_{x}(t) j_{x}(0)\right\rangle$ in the presence of the electric field $\left(E_{B}=\right.$ $\left.0.1 \mathrm{E}_{\mathrm{f}}, \Delta_{\mathrm{R}}=0\right)$

orbital Rashba term $\mathrm{H}_{\mathrm{OR}}$ the current density operator in $x$ direction has also contributions from the pseudospin in $z$ direction as

$$
j_{x}=i[H, x]=\frac{-e}{\hbar}\left(\hbar v_{f} \sigma_{x}+V_{O R} E_{y} \sigma_{z}\right) \text {. }
$$

However, the current density operator in $y$ direction is still proportional to $\sigma_{y}$

$$
j_{y}=\mathfrak{i}[H, y]=-e v_{f} \sigma_{y}
$$

We also showed in Sec. 3.1.I that an in-plane electric field can affect the Landau levels in graphene and at sufficiently large electric field $\left(E=B v_{F}\right)$ leads to the collapse of Landau levels.

In this section, we study how the in-plane electric field can affect the Rabi oscillations in Landau quantized graphene. In this part we show our numerical results by calculation of current-current correlation functions $\left\langle j_{x}(t) j_{x}(0)\right\rangle$ and $\left\langle j_{x}(t) j_{y}(0)\right\rangle$ using Matlab and quantum optic toolbox. In our calculations, we assume that the initial two level system state is localized on one of the sublattices, 


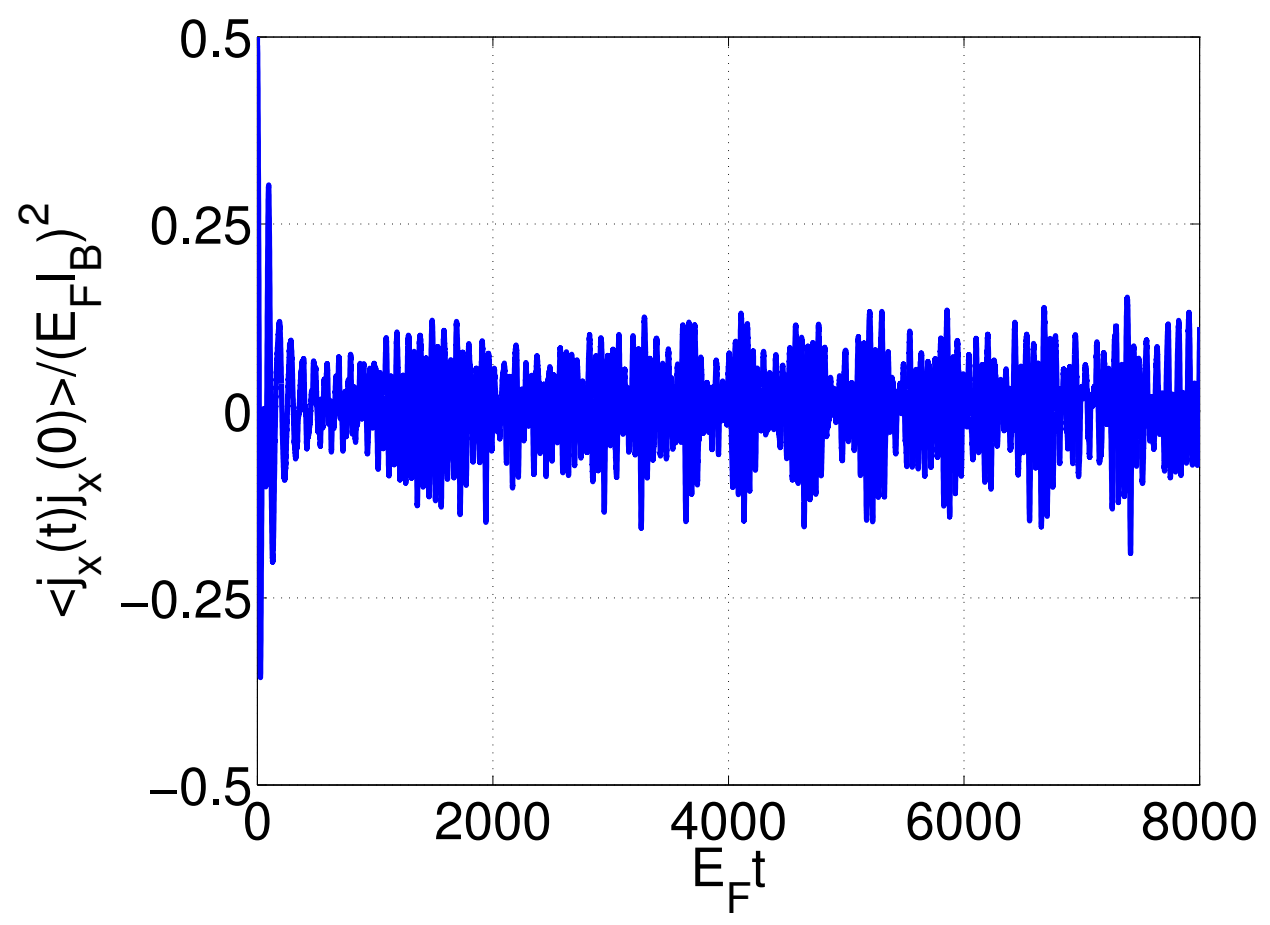

Figure 35: Time evolution of $<j_{x}(t) j_{x}(0)>$ in the presence of an electric field $\left(E_{B}=\right.$ $\left.0.15 \mathrm{E}_{\mathrm{f}}, \Delta_{\mathrm{R}}=0\right)$

and the field state is a coherent state. The average boson number is chosen to be $\bar{n}=25$. The truncation number in our calculation is $N=100$. The initial state of the system is therefore

$$
|\psi\rangle=\sum_{n=0}^{N} e^{-|\alpha|^{2} / 2} \frac{\alpha^{n}}{\sqrt{n !}}|n\rangle \otimes|\sigma\rangle
$$

where $\alpha=\sqrt{\bar{n}}$ and $n$ being the number of Landau levels. This initial state is being used for calculating correlation functions.

In Fig. 33 the time evolution of the correlation function $\left\langle j_{x}(t) j_{x}(0)\right\rangle$ in the absence of an electric field, shows that Rabi oscillations in quantum optics can be observed for Landau-quantized Dirac fermions. The revival time depends on the square root of the mean number of bosons in the field. Applying an in-plane electric field in a given direction, e.g. y, can influence the Rabi oscillations.

As is shown in Fig. 34, the revival time is influenced by applying the in-plane electric field. As the strength of the electric field increases, the amplitude of oscil- 


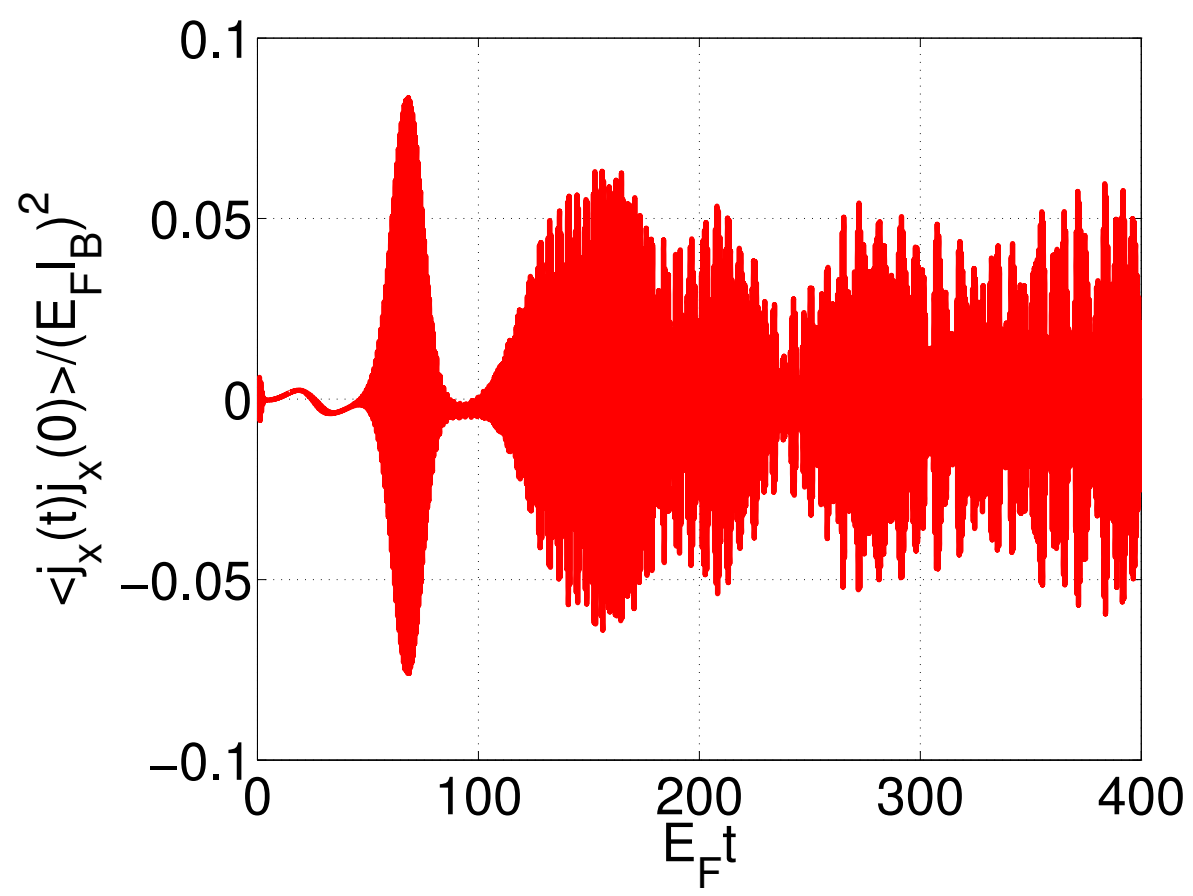

Figure 36: Time evolution of $\left\langle j_{x}(t) j_{y}(0)\right\rangle$ in the presence of the electric field $\left(E_{B}=\right.$ $\left.0.1 \mathrm{E}_{\mathrm{F}}, \Delta_{\mathrm{R}}=0.0\right)$

lations decreases, and higher electric fields can destroy the collapse and revival patterns of oscillations as shown in Fig. 35 .

The correlation function $\left\langle j_{x}(t) j_{y}(0)\right\rangle$ in the absence of an in-plane electric field, when a coherent state is prepared in graphene, is zero. However by applying in-plane electric field oscillations occur. The time evolution of the correlation function $\left\langle j_{x}(t) j_{y}(0)\right\rangle$ is shown in Fig. 36 and Fig. 37 for $E_{B}=0.1$ and $E_{B}=0.15$ respectively.

We have also considered the orbital-Rashba term $\Delta_{R}$ in our calculations to see how it can affect the Rabi oscillations. However, since the effect of this term on Landau levels is negligible compared to the term proportional to $E_{B}$ in Eq. 180, we found that this term hardly changes the Rabi oscillation patterns for $\left\langle j_{x}(t) j_{y}(0)\right\rangle$ and $\left\langle j_{x}(t) j_{x}(0)\right\rangle$. 


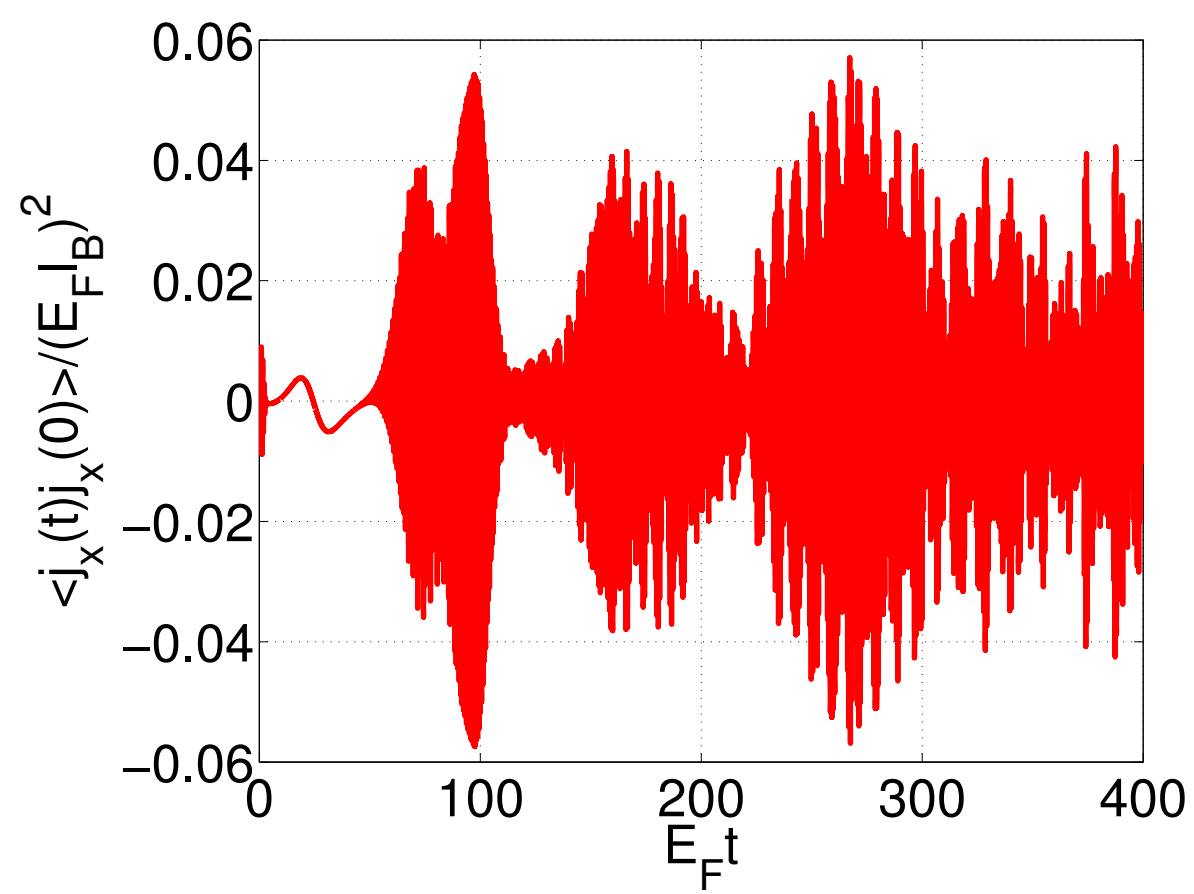

Figure 37: Time evolution of $\left\langle j_{x}(t) j_{y}(0)>\right.$ in the presence of an electric field $\left(E_{B}=\right.$ $\left.0.15 \mathrm{E}_{\mathrm{f}}, \Delta_{\mathrm{R}}=0.0\right)$

\subsection{SUMMARY AND CONCLUSION}

In the present section, we investigated the novel orbital Rashba effect due to an in-plane electric field in graphene coupling the orbital motion of an electron with the pseudospin degree of freedom. The Landau levels in graphene in the presence of in-plane electric field show a collapse when $E=B v_{F}$. By using perturbation theory, we study the effect of orbital Rashba on the Landau levels of graphene. Our calculations show that the orbital Rashba term does not affect the Landau levels to first order. However, second-order perturbation is not zero for electric fields $0<\mathrm{E}<\mathrm{B} v_{\mathrm{F}}$ and causes electron and hole Landau levels to repel each other. When $E=0$ or $E=B v_{F}$ the correction due to orbital Rashba is zero. Our calculations also show that the Landau level with $n=0$ is not affected even by second-order perturbation theory.

We also study how the orbital Rashba effect can influence the minimal conductivity of graphene. We have calculated the AC conductivity of graphene in the 
presence of an orbital Rashba term using the Kubo formula in the linear response regime. We show that this term does not affect conductivity due to intraband transitions. However, our calculations show that due to the orbital Rashba effect, the longitudinal conductivity in clean graphene system at zero temperature and finite frequency is not independent of the frequency. This result is in contrast to previous studies that show minimal conductivity in the absence of disorder and with the finite frequency has a universal value independent of the frequency.

Finally, we study the effect of in-plane electric fields on Rabi oscillations in the Landau quantized graphene system by calculating longitudinal and transverse current-current correlation functions numerically. We show that an in-plane electric field can destroy the collapse and revival patterns in Rabi oscillations. Our calculations show that the orbital Rashba effect does not have a significant influence on Rabi oscillations. 


\section{4}

CHIRAL TUNNELING IN BLG: EFFECT OF RASHBA

SPIN-ORBIT COUPLING

\subsection{INTRODUCTION}

As we previously discussed in Sec. 1.4, chiral tunneling in monolayer graphene shows that electrons with normal incidence can fully transmit through a barrier regardless of the width and height of the barrier. However this transmission is forbidden in bilayer graphene and there is a perfect back scattering for normally incident electrons. There are also so-called magic angles for both cases in which the transmission is perfect (Katsnelson et al., 2006; Allian and Fuchs, 2011; Tudorovskiy and Katsnelson, 2012; Cheianov and Falḱko, 2006).

Since Rashba SOC couples the spin and pseudospin degree of freedom, it can affect Klein tunneling. For example it has been shown that the low energy effective dispersion relation for monolayer graphene in the presence of Rashba SOC is similar to that for spin degenerate BLG (Yamakage et al., 2009; Rashba, 2009; Bercioux and Martino, 2010), hence transport properties through npn junctions for both systems show the same features (M. Liu and Richter, 2012). The band structure of BLG in the presence of Rashba SOC shows unique characteristics that are not seen in MLG or other conventional 2D systems (see Sec. 1.6). Here in this chapter, we investigate the influence of Rashba SOC on transport properties of a BLG system by studying chiral tunneling through npn and np junctions. 


\subsection{BAND STRUCTURE OF BILAYER GRAPHENE IN PRESENCE OF RASHBA SOC}

We consider a clean BLG in the $x y$ plane and assume that electron-electron interactions are negligible such that the system can be well defined using a single electron model. The low energy effective Hamiltonian for this system is given by:

$$
\mathrm{H}=\frac{-1}{2 \mathrm{M}}\left(\pi_{+}^{2} \sigma_{+}+\pi_{-}^{2} \sigma_{-}\right)-i v_{\mathrm{B}}\left(\pi_{+} \sigma_{+} \mathrm{s}_{-}-\pi_{-} \sigma_{-} \mathrm{s}_{+}\right)+\mathrm{V},
$$

where $\pi_{ \pm}=\hbar\left(k_{x} \mp i k_{y}\right)$ and $\left(k_{x}, k_{y}\right)$ being the wave vector components, $\sigma_{ \pm}=$ $\frac{1}{2}\left(\sigma_{x} \pm i \sigma_{y}\right)$ and $s_{ \pm}=\frac{1}{2}\left(s_{x} \pm i s_{y}\right)$ with $\left(\sigma_{x}, \sigma_{y}\right)$ and $\left(s_{x}, s_{y}\right)$ are the ordinary Pauli matrices acting on the pseudo-spin and spin degree of freedom respectively. $M=0.035 m_{0}$ ( $m_{0}$ is the free electron mass) represents the effective mass of the charge carriers in bilayer graphene. The second term in Eq. 253 shows the effect of Rashba SOC with $v_{B}$ indicating its strength. The effective low energy Hamiltonian in the atomic basis $\left\{\chi_{A}^{\uparrow}, \chi_{A}^{\downarrow}, \chi_{B}^{\uparrow}, \chi_{B}^{\downarrow}\right\}$ where $A / B$ denote different sublattices, is given by:

$$
H=\left(\begin{array}{cccc}
V & 0 & -\frac{\pi_{+}^{2}}{2 M} & 0 \\
0 & V & -i v_{B} \pi_{+} & -\frac{\pi_{+}^{2}}{2 M} \\
-\frac{\pi_{-}^{2}}{2 M} & i v_{B} \pi_{-} & V & 0 \\
0 & -\frac{\pi_{-}^{2}}{2 M} & 0 & V
\end{array}\right) .
$$

The corresponding eigenvalue and eigenfunction of Eq. 254 are given by:

$$
E_{\mu s}(k)=\frac{\mu}{2} \hbar k\left(\sqrt{v_{B}^{2}+\frac{\hbar^{2} k^{2}}{M^{2}}}-s v_{B}\right)+V,
$$

and

$$
\left|\psi_{k^{s}}^{\mu}\right\rangle=\left(\begin{array}{c}
-i \mu s e^{-3 i \phi} \\
-g_{v_{B}}(k) \mu e^{-2 i \phi} \\
i g_{v_{B}}(k) s e^{-i \phi} \\
1
\end{array}\right)
$$


where $\mu$ and $s$ with the value \pm 1 are band indices, indicating electron/hole

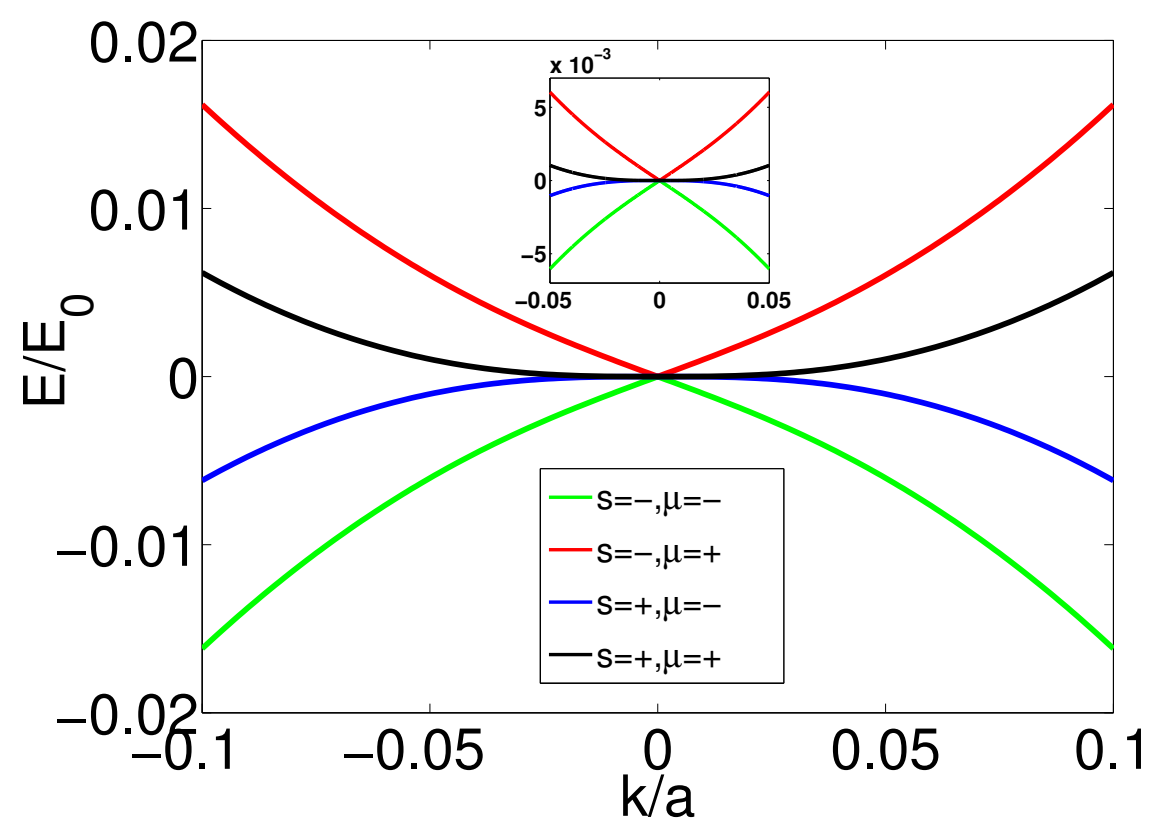

Figure 38: Dispersion relation of a BLG in the presence of Rashba SOC with strength $\bar{v}_{\mathrm{B}}=0.02$. Different colors indicate bands with different indices. Here $s$ and $\mu$ indicate spin and electron/hole indices respectively.

branch and spin chirality respectively, and we used the abbreviation $g_{v_{\mathrm{B}}}(k) \equiv$ $\frac{-s v_{B}+\sqrt{v_{B}^{2}+\frac{\hbar^{2} k^{2}}{M^{2}}}}{\frac{\hbar k}{M}}$.

\subsubsection{Dimensionless relations}

Considering $\mathrm{E}_{0}=\frac{\hbar^{2}}{2 \mathrm{Ma}^{2}}$ as the energy scale, we can write the dimensionless Hamiltonian as

$$
\overline{\mathrm{H}} \equiv \mathrm{H} / \mathrm{E}_{0}=-\left(\overline{\mathrm{k}}_{+}^{2} \sigma_{+}+\overline{\mathrm{k}}_{-}^{2} \sigma_{-}\right)-i \bar{v}_{\mathrm{B}}\left(\overline{\mathrm{k}}_{+} \sigma_{+} S_{-}-\overline{\mathrm{k}}_{-} \sigma_{-} S_{+}\right)+\overline{\mathrm{V}},
$$

where

$$
\bar{v}_{\mathrm{B}} \equiv \frac{\hbar}{\mathrm{a} \mathrm{E}_{0}} v_{\mathrm{B}}, \quad \overline{\mathrm{k}}_{-} \equiv \mathrm{k}_{-} \mathrm{a}, \quad \overline{\mathrm{k}}_{+} \equiv \mathrm{k}_{+} \mathrm{a}, \quad \overline{\mathrm{v}} \equiv \frac{\mathrm{V}}{\mathrm{E}_{0}},
$$


in which $\mathrm{a}=0.246 \mathrm{~nm}$ is graphene lattice parameter. The dispersion relation can therefore be written in dimensionless form as

$$
\overline{\mathrm{E}}_{\mu s}(\mathrm{k})=\frac{\mu}{2} \overline{\mathrm{k}}\left(\sqrt{\bar{v}_{\mathrm{B}}^{2}+4 \overline{\mathrm{k}}^{2}}-s \bar{v}_{\mathrm{B}}\right)+\overline{\mathrm{V}}
$$

In Fig. 38, the energy dispersion relation of bilayer graphene in the presence of Rashba spin-orbit coupling with $\bar{v}_{\mathrm{B}}=0.02$ has been shown. Since Rashba SOC does not break the inversion symmetry, it does not induce a gap in the band structure. However, spin degeneracy of the system is broken and there is a spin splitting in the band structure.

\subsubsection{Band structure in different regimes}

In the presence of Rashba SOC $\left(v_{\mathrm{B}} \neq 0\right)$ the degeneracy of the band structure is broken, and it results in four different subbands which are indicated by the subband indices $s$ and $\mu$. Regarding the strength of $\nu_{B}$ we have two different regimes:

1. Weak Rashba SOC $\left(\frac{\hbar^{2} k^{2}}{M^{2}}>>v_{B}^{2}\right)$ :

The dispersion relation can be shown approximately by $E_{\mu s}(k) \simeq \mu\left(\frac{\hbar^{2} k^{2}}{2 M}-\frac{s}{2} \hbar k v_{B}\right)$ which implies that the behavior of subbands are parabolic.

2. Strong Rashba SOC $\left(\frac{\hbar^{2} k^{2}}{M^{2}}<<v_{B}^{2}\right)$ :

In this regime the band structure has different forms for subbands with different band index $s$. When $s=+1$ (outermost subband) the dispersion relation has the approximate form as $E_{\mu s}(k) \simeq \mu \frac{\hbar^{3} k^{3}}{4 M^{2} v_{B}}$ which shows that this subband has a $k$ cubic spectrum. For $s=-1$ (innermost subband) the energy-momentum relation has a linear shape as $E_{\mu s}(k) \simeq \mu \hbar k v_{B}$. 


\subsubsection{Spin polarization}

In the presence of Rashba spin-orbit coupling, spin polarization $\langle\vec{S}\rangle_{\mu s}=\left\langle\psi_{\mathrm{ks}}^{\mu}|\vec{S}| \psi_{\mathrm{ks}}^{\mu}\right\rangle$ in MLG and BLG in contrast to conventional semiconductors is momentum dependent and is given by

$$
\begin{aligned}
& \left\langle S_{x}\right\rangle_{\mu s}=4 s g_{v_{\mathrm{B}}}(k) \sin (\phi), \\
& \left\langle S_{y}\right\rangle_{\mu s}=-4 s g_{v_{\mathrm{B}}}(\mathrm{k}) \cos (\phi), \\
& \left\langle S_{z}\right\rangle_{\mu s}=0,
\end{aligned}
$$

where $g_{v_{B}}(k) \equiv \frac{-s v_{B}+\sqrt{v_{B}^{2}+\frac{\hbar^{2} k^{2}}{M^{2}}}}{\frac{\hbar k}{M}}$. The components of the spin polarization for unbiased bilayer graphene can be written in a compact form by (Mireles and Schliemann, 2012; Rashba, 2009)

$$
\langle\overrightarrow{\mathrm{S}}\rangle_{\mu \mathrm{s}}=\frac{\mathrm{s}}{M \sqrt{v_{\mathrm{B}}^{2}+\mathrm{k}^{2} / \mathrm{M}^{2}}}(\hat{z} \times \mathbf{k}),
$$

which is the same as the case of monolayer graphene with Rashba SOC interaction.

Therefore, in unbiased BLG $\langle\vec{S}\rangle_{\mu s}$ lies in the BLG plane and as long as $v_{\mathrm{B}}$ is finite, spin is no longer a good quantum number since $|\langle\vec{S}\rangle| \neq 1$ and in fact $|\langle\vec{S}\rangle|$ has $\mathrm{k}$-dependent oscillations. The orientation of the spin polarization is always perpendicular to the momentum direction that is $\vec{S} \cdot \vec{k}=0$. Hence, we can define two types of spin polarization with respect to the band index s, which are clockwise with $s=+1$ and anti-clockwise with $s=-1$.

\subsection{N-P-N JUNCTION WITH SO-SO-SO INTERFACES}

In order to investigate the chiral tunnelling in BLG with Rashba SOC interaction, we first consider a n-p-n BLG junction with a finite and uniform Rashba SOC inside and outside the barrier. The potential barrier $V$ has a rectangular shape assumed as

$$
V(x)=\left\{\begin{array}{ccc}
V_{0} & \text { for } & 0<x<L \\
0 & \text { otherwise }
\end{array}\right.
$$


where $L$ and $V_{0}$ determine the width and the height of the barrier respectively. Here assuming a potential barrier with sharp edges requires that the Fermi wavelength be larger than the lattice constant over which the potential changes (Cheianov and Falko, 2006). We solve the scattering problem for a quasiparticle tunneling through a barrier of the height $V_{0}$ and the width $L$ as sketched in Fig. 39. $E$ and $\phi$ represent energy of the particle and its angle of incidence respectively.

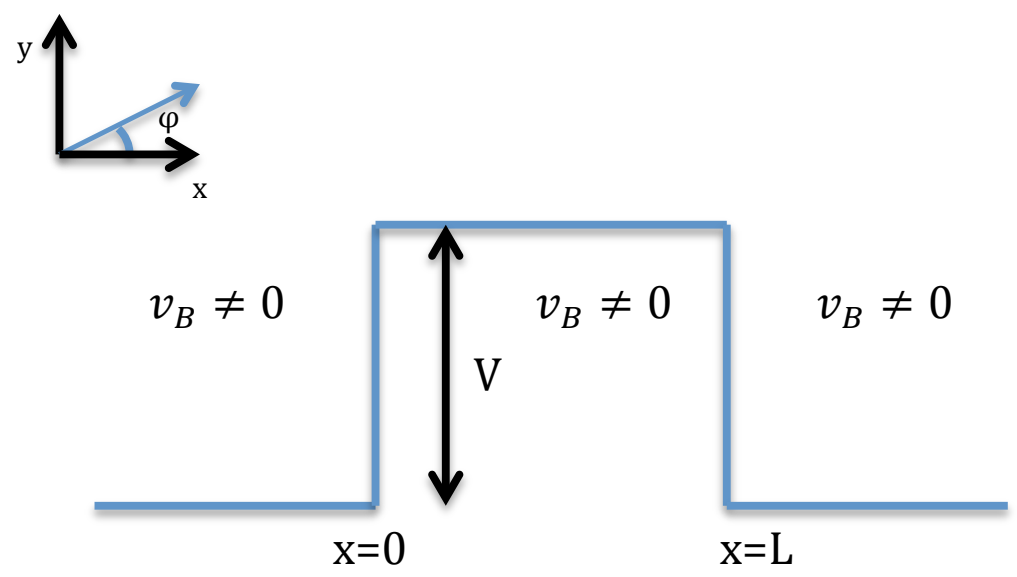

Figure 39: The energy profile of a BLG tunnelling barrier in the presence of Rashba spinorbit coupling $v_{\mathrm{B}}$

Due to translational invariance in $y$ direction, $k_{y}$ is conserved throughout the system and the scattering problem can be considered effectively as a one dimensional problem. Therefore, the solution of the Hamiltonian (254) for an incident electron with spin chirality $s$ and angle of incidence $\tan ^{-1}\left(\frac{k_{y}}{k_{x}}\right)=\phi$ is given by 
$\psi(x, y)=\chi(k) e^{i k_{x}^{s} x} e^{i k_{y} y}$ where $\chi(k)$ is a four-component spinor. The scattering states in different regions are given by

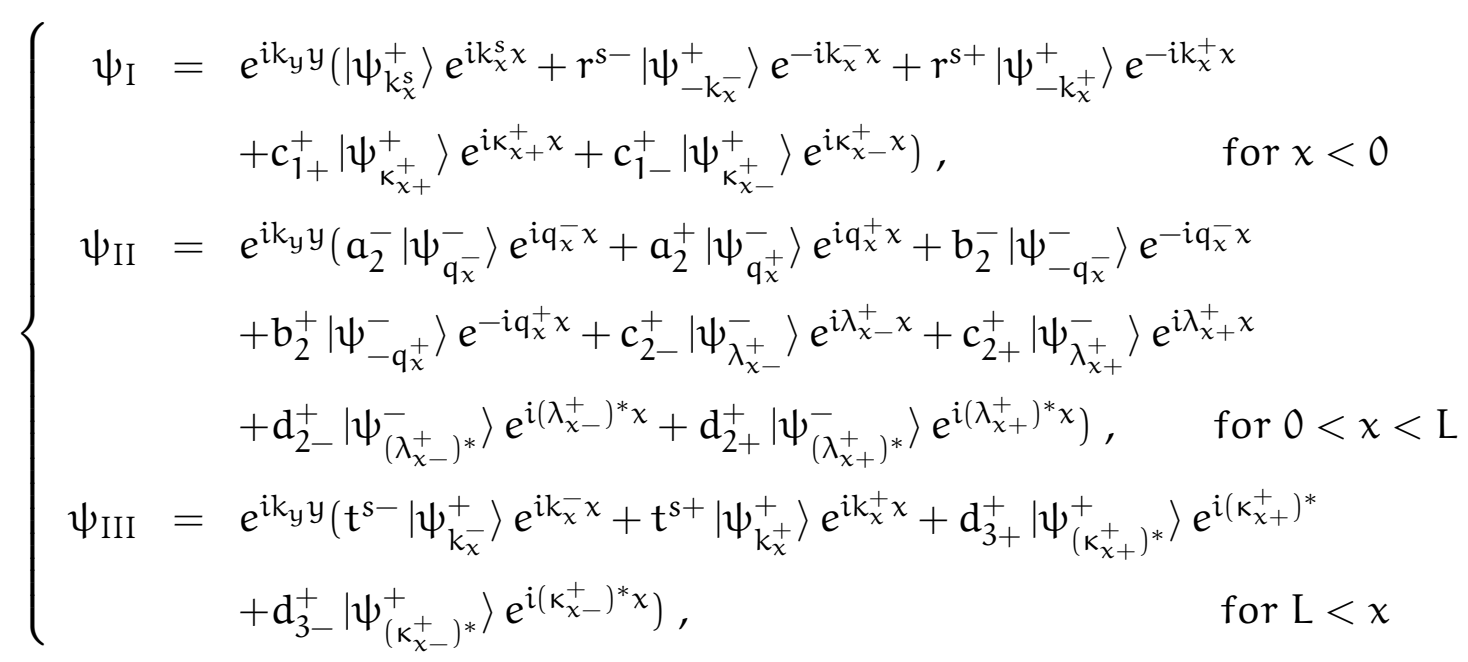

where $r^{s s^{\prime}}$ and $t^{s s^{\prime}}$ are respectively the complex amplitudes of the reflected and transmitted waves with spin chirality $s^{\prime}$ when the incident wave has the spin chirality s. By solving Eq. (255) for a given energy $E$ and momentum $k_{y}$ one can find the wave vectors corresponding to propagating and evanescent modes for $n$ and $p$ regions. For $s=-1$ there are two real wave vectors $\pm k_{x}^{-}$corresponding to two propagating waves $e^{ \pm i k_{\chi}^{-} \chi}$, whereas for $s=+1$, besides the two real wave vectors $\pm k_{x}^{+}$for propagating waves $e^{ \pm i k_{x}^{+} \chi}$, there are also four complex (not purely imaginary) wave vectors of the form $\kappa_{x \pm}^{+}= \pm \alpha-i \beta$ ( $\alpha$ and $\beta$ are real and positive) and $\left(\kappa_{x \pm}^{+}\right)^{*}$, corresponding to the evanescent waves $e^{i \kappa_{x \pm}^{+} x}$ and $e^{i\left(\kappa_{x \pm}^{+}\right)^{*} x}$.

For a rectangular potential barrier, the unknown coefficients can be found from the continuity of the wave function

$$
\left\{\begin{array}{c}
\left.\psi_{\mathrm{I}}\right|_{x=0}=\left.\psi_{\mathrm{II}}\right|_{x=0} \\
\left.\psi_{\mathrm{II}}\right|_{x=\mathrm{L}}=\left.\psi_{\mathrm{III}}\right|_{x=\mathrm{L}}
\end{array}\right.
$$

and the probability flux

$$
\left\{\begin{array}{c}
\left.\hat{v}_{x} \psi_{\mathrm{I}}\right|_{x=0}=\left.\hat{v}_{x} \psi_{\mathrm{II}}\right|_{x=0} \\
\left.\hat{v}_{x} \psi_{\mathrm{II}}\right|_{x=\mathrm{L}}=\left.\hat{v}_{x} \psi_{\mathrm{III}}\right|_{x=\mathrm{L}}
\end{array}\right.
$$

in different interfaces, where the velocity operator in $x$-direction $\hat{v}_{x}$ is given by

$$
\hat{v}_{x}=\frac{-1}{M}\left(\pi_{+} \sigma_{+}+\pi_{-} \sigma_{-}\right)-i v_{B}\left(\sigma_{+} S_{-}-\sigma_{-} S_{+}\right) .
$$


For more technical details of the derivation of the velocity operator in the presence of Rashba SOC refer to Appendix A.I.

\subsubsection{Kinematics of scattering}

In analogy with conventional Rashba SOC in semiconductors, the spin polarization $\vec{S}$ in BLG is always perpendicular to the momentum $\vec{k}$, and the band index $s=+1(s=-1)$ corresponds to clockwise (anti-clockwise) spin chirality. For a given energy $E_{f}$, there are two Fermi wavelengths $k_{f}^{>}$and $k_{f}^{<}$corresponding to clockwise and anti-clockwise spin chirality respectively. Fig. 40 shows Fermi circles for a given Fermi energy and bias voltage in BLG. When $V>E_{f}$, the BLG tunnel barrier is a n-p-n junction. For $V>2 E_{f}$, the Fermi circles of the barrier are always bigger than the regions where $V=0$. It is obvious that for $\phi>\arcsin \left(\frac{k_{f}^{<}}{k_{f}^{>}}\right)$ there is only one channel for electrons coming from Fermi circle $k_{f}^{>}$to be transmitted into. For $\mathrm{E}_{\mathrm{f}}<\mathrm{V}<2 \mathrm{E}_{\mathrm{f}}$ the Fermi circles of the barrier are smaller than the Fermi circles of the regions where there is no applied gate voltage. So when $V \gtrsim E_{f}$, there is a small angle of incidence $\phi$ in which transmission probability is not zero. As it is shown in Fig. 41, increasing the strength of the Rashba SOC leads to an increase in the difference between the radius of the two Fermi circles. Hence in the regime of $V \gtrsim E_{f}$, increasing $\bar{v}_{B}$ results in overlap between $k_{f}^{<}$in the incidence region and $k_{f}^{>}$of the barrier region. This feature leads to having non-zero transmission probabilities for the larger angle of incidence.

\subsubsection{Numerical results for npn junction}

Fig. 42 shows the chiral tunneling in a clean BLG in the absence of Rashba SOC. In order to make a comparison with the results reported by Katsnelson et al. (2006), the height of the barrier is chosen to be $V=0.0043 \mathrm{E}_{0}(50 \mathrm{meV})$ and $V=0.0086 \mathrm{E}_{0}$ (100 $\mathrm{meV})$, the width of the barrier is $W=406.33 \mathrm{a}(100 \mathrm{~nm})$ and energy of the incident electron is $E_{f}=0.00146 E_{0}(17 \mathrm{meV})$ where $E_{0} \equiv \frac{\hbar^{2}}{2 \mathrm{Ma}^{2}}$ and $a=2.46 \AA$ 

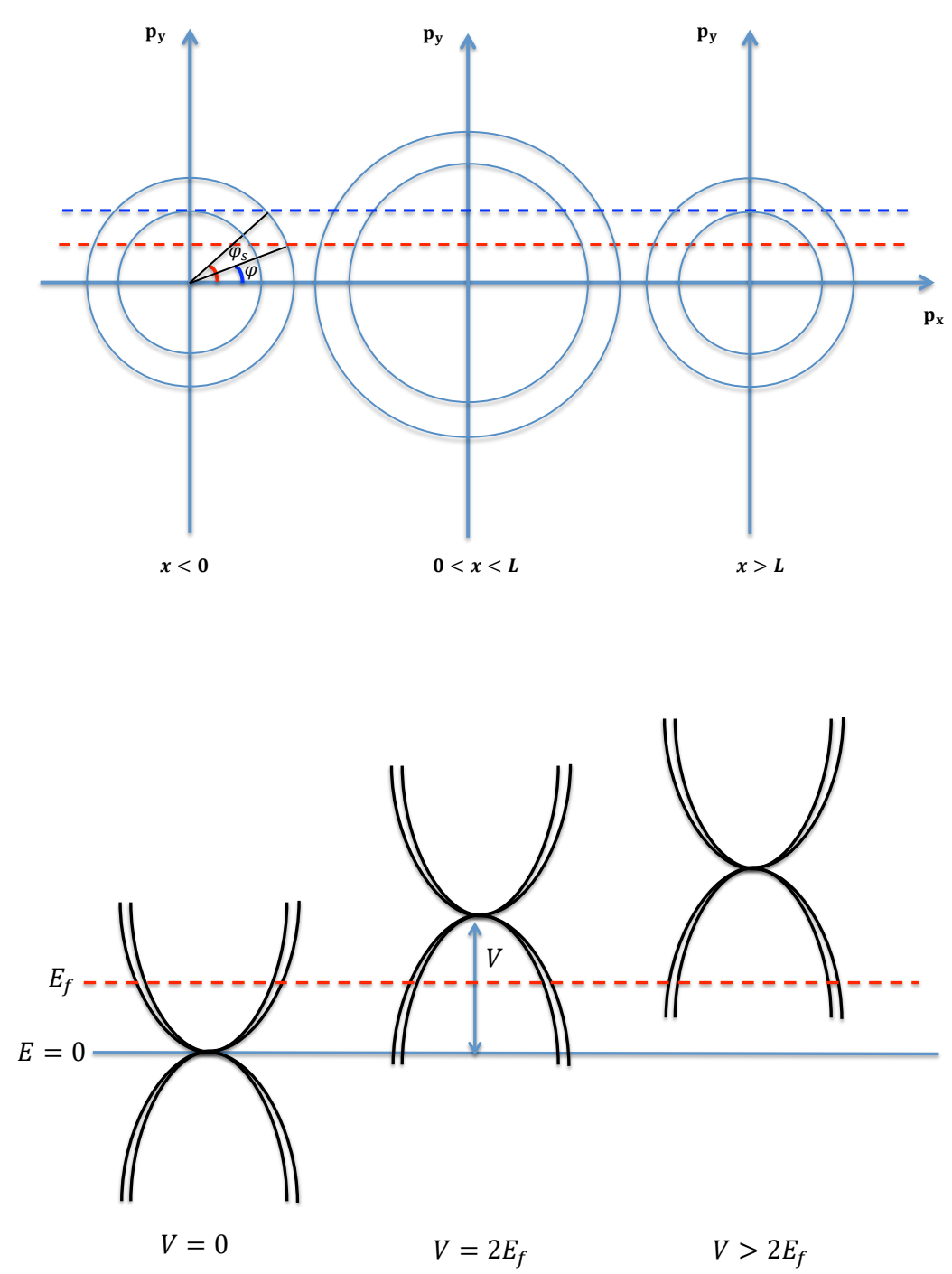

Figure 40: Kinematic scattering in a n-p-n BLG junction in the presence of Rashba SOC. Fermi circles are related to electrons with different chiralities. It is clear that when the angle of the incident electrons are larger than $\phi_{s}$ there is only one channel for the incident electrons from the outer Fermi circle to be transmitted into. For $\mathrm{V}>2 \mathrm{E}_{\mathrm{f}}$, the Fermi circles are bigger than the Fermi circles of the incidence and transmitted regions. 


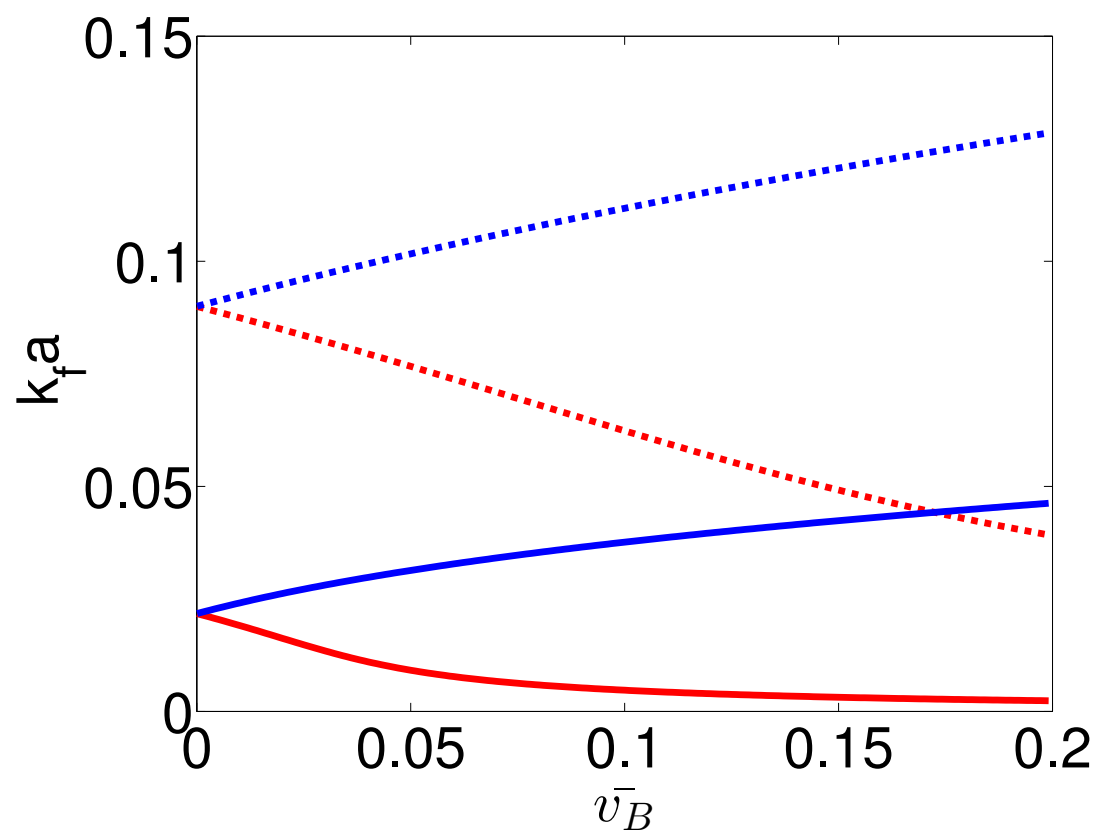

Figure 41: Radius of the Fermi circles $k_{f}^{>}$(clockwise spin chirality) and $k_{f}^{<}$(anticlockwise spin chirality) versus the strength of the Rashba SOC, $\bar{v}_{\mathrm{B}}$, for $\mathrm{V}=0$ (dashed lines) and $\mathrm{V}=50 \mathrm{meV}$ when the energy of the incident electrons is $E_{f}=45 \mathrm{meV}$.

the graphene lattice parameter. These results are in agreement with the ones reported by Katsnelson et al. (2006) that show normal transmission is forbidden for a rectangular barrier and there are magic angles in which transmission is perfect regardless of the height and width of the barrier. This effect is due to conservation of the pseudo-spin in graphene. In the presence of Rashba SOC, spin and pseudospin are coupled and this can affect the transmission probability. Fig. 43 shows the transmission probability of an incident electron with specific spin chirality with Rashba SOC strength $\bar{v}_{B}=0.01$. The transmission probability $\mathrm{T}_{\mathrm{ss}^{\prime}}$ corresponds to an incoming electron with spin chirality $\mathrm{s} \equiv \pm$ and transmitted by an outgoing electron with spin chirality $s^{\prime} \equiv \pm$ and it is defined as $\mathrm{T}_{s s^{\prime}}=\frac{J_{s^{\prime}}^{\operatorname{tran}}\left(t^{s s^{\prime}} t^{s s^{\prime *}}\right)}{J_{s}^{\text {inc }}}$ where $J_{s}^{\text {inc }}\left(J_{s}^{\text {tran }}\right)$ are the probability currents of an incident (transmitted) electron with spin chirality s. The total transmission probability $T_{\text {total }}$ is the sum of all transmission probabilities defined as $T_{\text {total }}=\sum_{s, s^{\prime}= \pm} T_{s s^{\prime}}$. In Fig. 43, for angles of incidence with $|\phi|>\phi_{s}$ where $\phi_{s}= \pm 1.01$ radian, the 

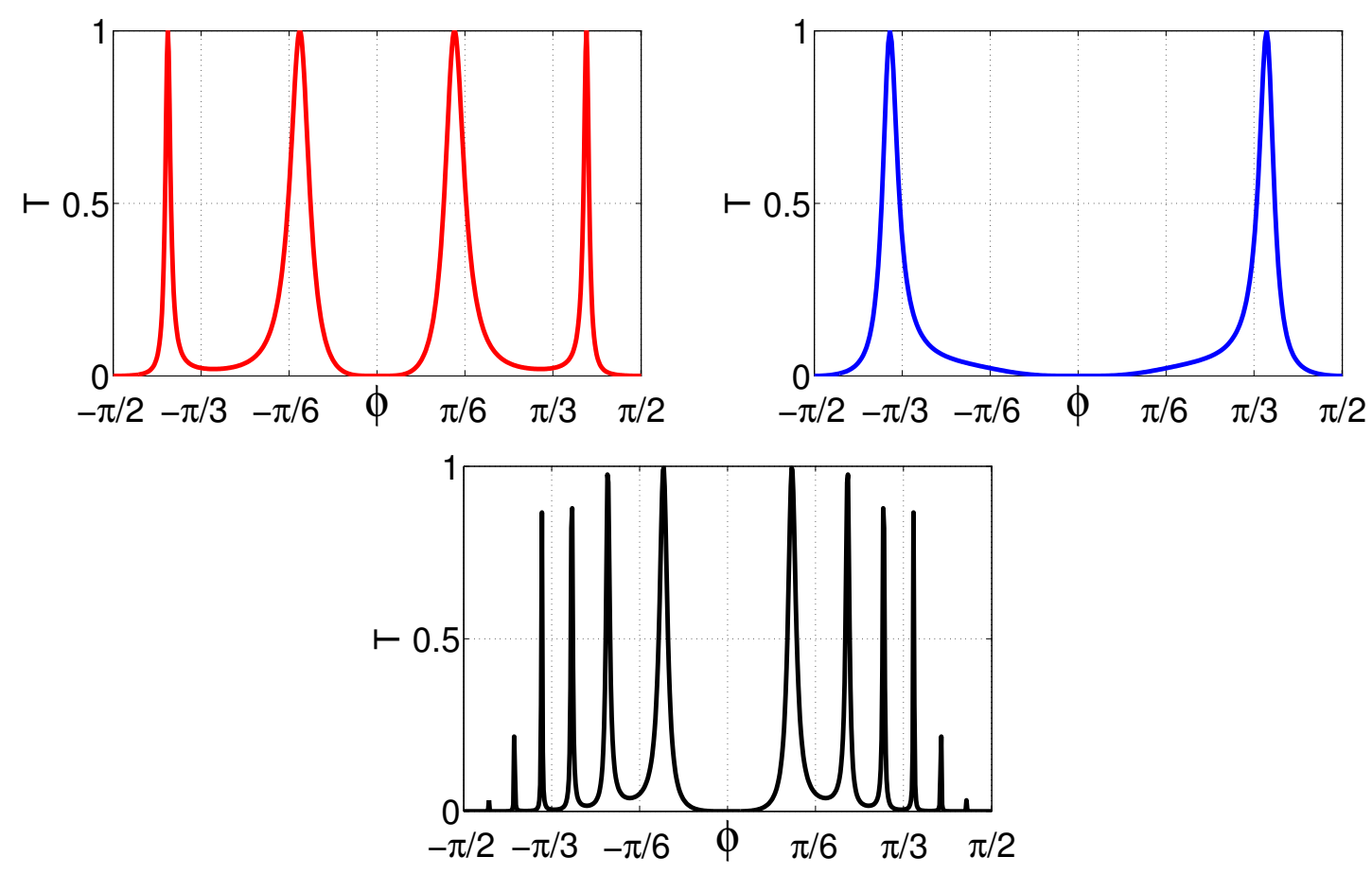

Figure 42: Transmission probability in n-p-n BLG junction versus angle of incidence $\phi$ in the absence of Rashba SOC with barrier height and Fermi energy V=10omeV and $E_{f}=17 \mathrm{meV}$ (blue), $V=50 m e V$ and $E_{f}=17 \mathrm{meV}$ (red) and $V=100 \mathrm{meV}$ and $\mathrm{E}_{\mathrm{f}}=50 \mathrm{meV}$ (black). 

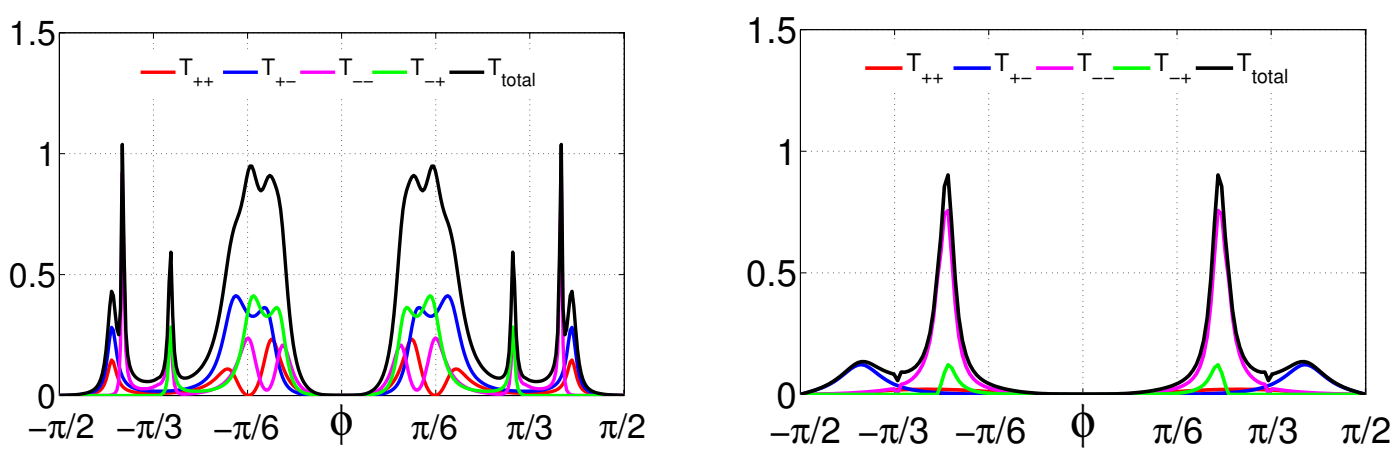

Figure 43: Transmission probability versus the angle of incidence $\phi$ for Rashba SOC strength $\bar{v}_{\mathrm{B}}=0.01$ and barrier height $\mathrm{V}=100 \mathrm{meV}$ (right) and $\mathrm{V}=50 \mathrm{meV}$ (left). Here $T_{s s^{\prime}}$ corresponds to an outgoing electron with spin chirality s' when the incident electron has spin chirality $s$. $T_{\text {total }}$ is the sum of all transmission probabilities with different spin chirality.

transmission probability $\mathrm{T}_{+-}$will be zero since there is only one channel for the electrons to be transmitted into. One can also see that in the presence of Rashba SOC, transmission is not perfect for any angle of incidence.

In the presence of Rashba spin-orbit coupling, spin polarization in MLG and BLG in contrast with ordinary semiconductors is momentum dependent. The components for the spin polarization in unbiased MLG and BLG is given by (Mireles and Schliemann, 2012; Rashba, 2009)

$$
\langle\mathbf{S}\rangle_{\mu \mathrm{s}}=\frac{\mathrm{s}}{\mathrm{M} \sqrt{v_{\mathrm{B}}^{2}+\mathrm{k}^{2} / \mathrm{M}^{2}}}(\hat{z} \times \mathbf{k})
$$

and in unbiased BLG, $\langle\mathbf{S}\rangle_{\mu \mathrm{s}}$ lies in the BLG plane furthermore, as long as $\nu_{\mathrm{B}}$ is finite, spin is no longer a good quantum number and $|\langle\mathbf{S}\rangle| \neq 1$. Due to the momentum dependence, spin polarization of the incident waves from branches with different spin chirality s do not cancel each other. This causes the incident wave in our problem to become spin polarized. This spin polarization increases with the increase of the Rashba SOC strength $\bar{v}_{B}$ due to the increase in spin splitting which results in momentum separation for a given incident energy $E_{f}$.

Here we study the effect of Rashba SOC on the transmission probability through a BLG n-p-n junction. The height of the barrier and Fermi energy are chosen to be 

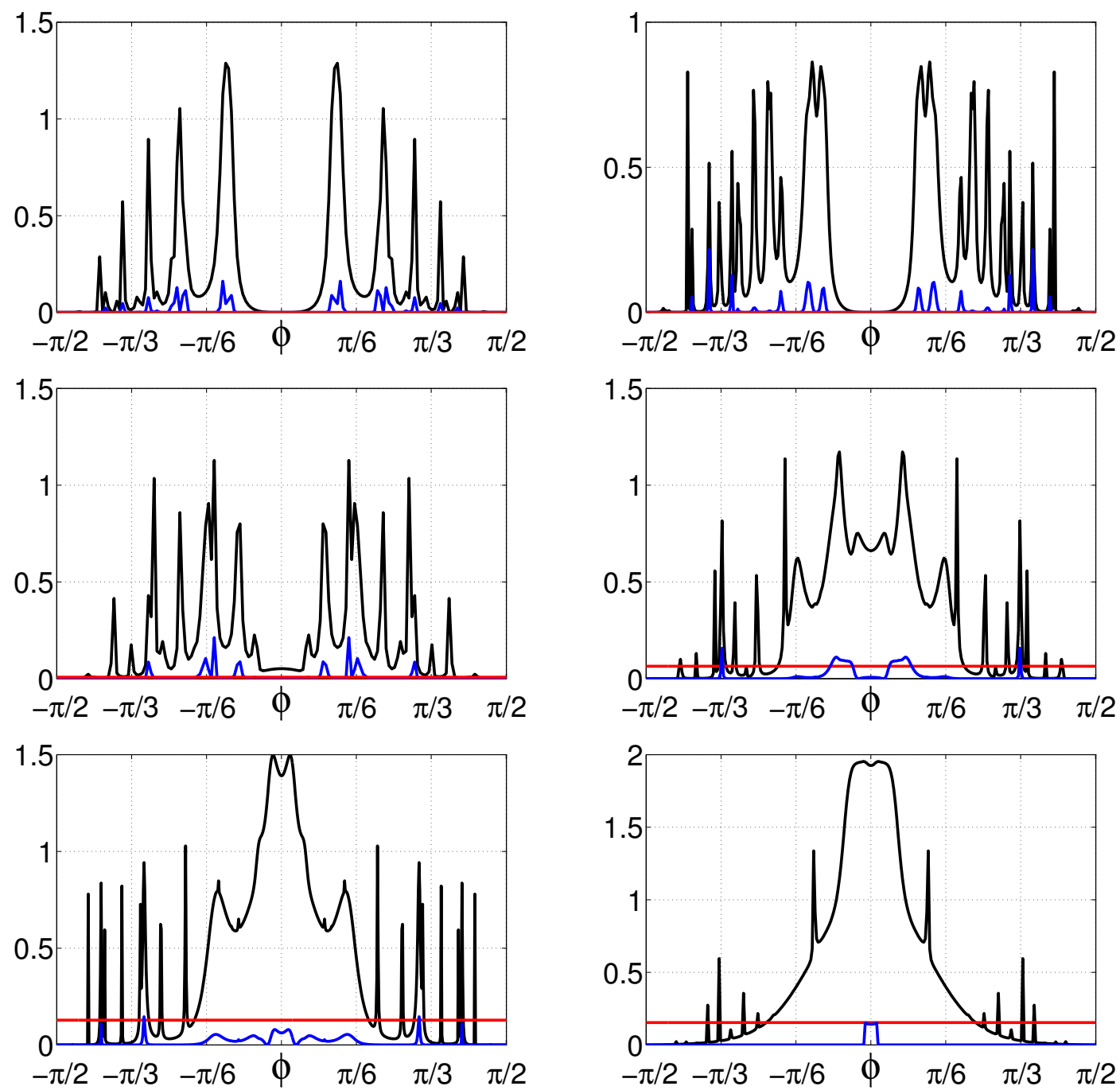

Figure 44: Angle resolved transmission probability $\mathrm{T}_{\text {total }}$ (black line) and spin polarization $\left\langle\mathrm{S}^{2}\right\rangle$ for the incident (red line) and transmitted electrons (blue line) in a $n-p-n$ junction with width $100 \mathrm{~nm}$, height $100 \mathrm{meV}$ and Fermi energy $E_{f}=50$ meV with different Rashba SOC strength: $\bar{v}_{\mathrm{B}}=0.005$ (top left), $\bar{v}_{\mathrm{B}}=0.01$ (top right), $\bar{v}_{\mathrm{B}}=0.05$ (middle left), $\bar{v}_{\mathrm{B}}=0.08$ (middle right), $\bar{v}_{\mathrm{B}}=0.1$ (bottom left) and $\bar{v}_{\mathrm{B}}=0.5$ (bottom right) . 
$\mathrm{V}=100 \mathrm{meV}, \mathrm{E}_{\mathrm{f}}=50 \mathrm{meV}$ (Fig.. 44), $\mathrm{V}=50 \mathrm{meV}$ and $\mathrm{E}_{\mathrm{f}}=17 \mathrm{meV}$ (Fig. 45) and $\mathrm{V}=100$ $\mathrm{meV}, \mathrm{E}_{\mathrm{f}}=17 \mathrm{meV}$ (Fig. 46 ) respectively. For small $\bar{v}_{\mathrm{B}}$, in which the system is in the weak Rashba SOC regime $\left(4 \bar{k}^{2}>>\bar{v}_{B}^{2}\right)$, the transmission probability corresponds to the peaks decreases but peaks still appear for the same angle of incidence (see top row of Fig. 44,45,46). By increasing the Rashba SOC strength $\bar{v}_{\mathrm{B}}$, the system transfers into the intermediate regime in which peaks might appear in different angle of incidence. More importantly the system starts to have a transmission probability for the normal incidence $(T(0) \neq 0)$ and $T(0)$ increases with increasing Rashba SOC strength (see middle row of Fig. 44,45,46). For sufficiently large Rashba SOC strength, the system will be in the strong Rashba SOC regime $\left(4 \overline{\mathrm{k}}^{2}<<\bar{v}_{\mathrm{B}}^{2}\right)$. In this regime, the transmission probability for an angle of incidence around $\phi=0$ is dominant. This can be understood from the fact that for large values of $\bar{v}_{B}$, the linear part in the Hamiltonian (253) becomes dominant, and the system, like in the case of monolayer graphene, shows a large transmission probability for normal incidence (see bottom row of Fig. 44,45,46).

The spin polarization $\langle S\rangle^{2}=\left\langle S_{x}\right\rangle^{2}+\left\langle S_{y}\right\rangle^{2}$ for the incident (red line) and transmitted waves (blue line) are also depicted in Figs. 44,45,46. In order to calculate the spin polarization of the incident $\left(\langle S\rangle_{\text {incident }}^{2}\right)$ and outgoing $\left(\langle S\rangle_{\text {outgoing }}^{2}\right)$ waves we have used

$$
\begin{aligned}
& \left|\psi_{\text {inc }}\right\rangle=\sum_{s= \pm 1}\left|\psi_{k_{x}^{s}}^{+}\right\rangle e^{i k_{x}^{s} x}, \\
& \left|\psi_{\text {out }}\right\rangle=\sum_{s, s^{\prime}= \pm 1} t^{s s^{\prime}}\left|\psi_{k_{x}^{s^{\prime}}}^{+}\right\rangle e^{i k_{x}^{s^{\prime} x}},
\end{aligned}
$$

to numerically calculate

$$
\begin{aligned}
\langle S\rangle_{\text {incident }}^{2} & =\left\langle\psi_{\text {inc }}\left|S_{x}\right| \psi_{\text {inc }}\right\rangle^{2}+\left\langle\psi_{\text {inc }}\left|S_{y}\right| \psi_{\text {inc }}\right\rangle^{2}, \\
\langle S\rangle_{\text {outgoing }}^{2} & =\left\langle\psi_{\text {out }}\left|S_{x}\right| \psi_{\text {out }}\right\rangle^{2}+\left\langle\psi_{\text {out }}\left|S_{y}\right| \psi_{\text {out }}\right\rangle^{2} .
\end{aligned}
$$

In the weak Rashba SOC regime, the spin polarization of the incident waves is very small but still finite. However, as long as we are in the weak Rashba SOC regime, 

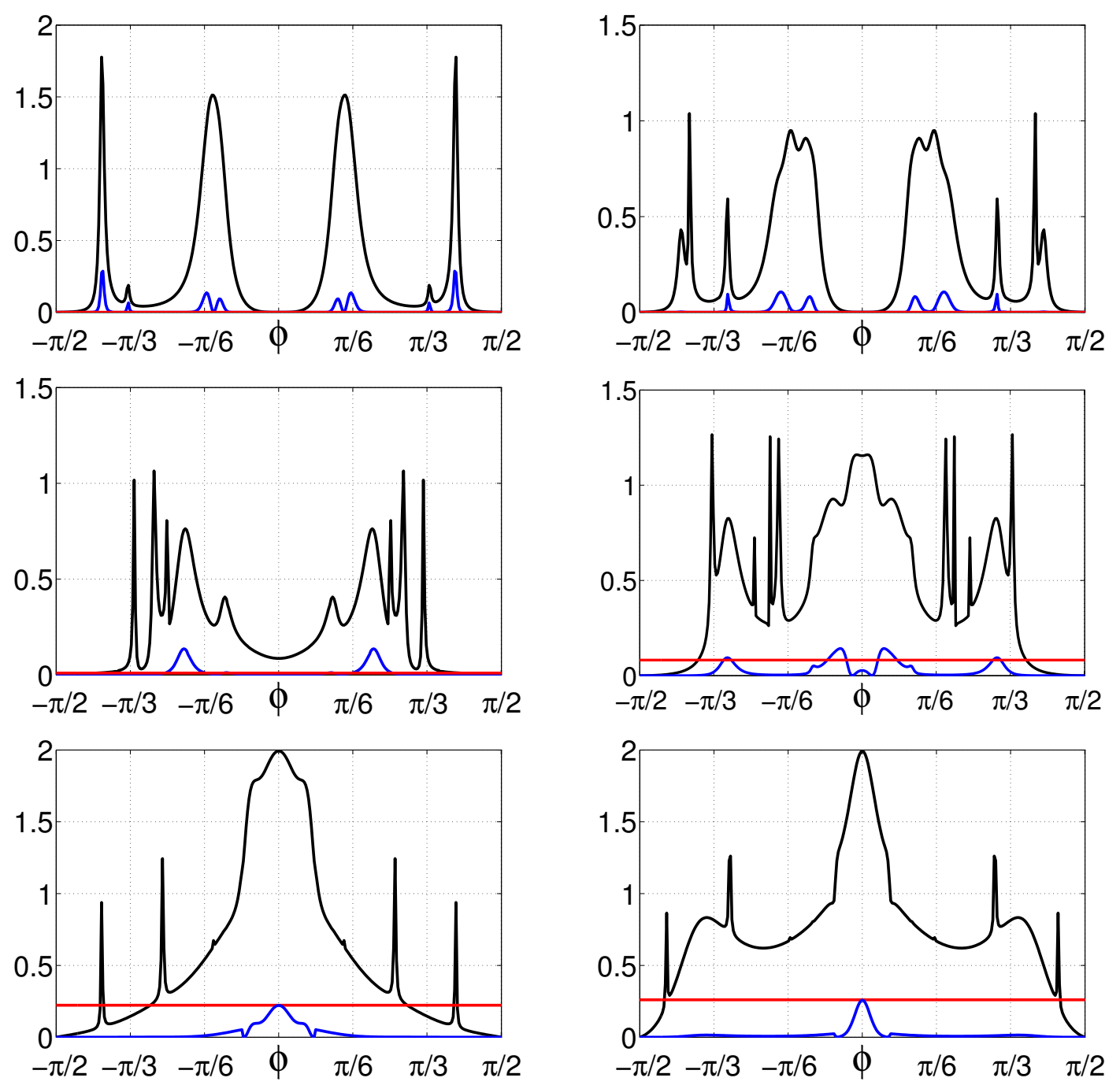

Figure 45: Angle resolved transmission probability $T_{\text {total }}$ (black line) and spin polarization $\left\langle\mathrm{S}^{2}\right\rangle$ for the incident (red line) and transmitted electrons (blue line) in a $n-p-n$ junction with width $100 \mathrm{~nm}$, height $50 \mathrm{meV}$ and Fermi energy $E_{f}=17$ meV with different Rashba SOC strength: $\bar{v}_{\mathrm{B}}=0.005$ (top left), $\bar{v}_{\mathrm{B}}=0.01$ (top right), $\bar{v}_{\mathrm{B}}=0.03$ (middle left), $\bar{v}_{\mathrm{B}}=0.05$ (middle right), $\bar{v}_{\mathrm{B}}=0.08$ (bottom left) and $\bar{v}_{\mathrm{B}}=0.1$ (bottom right) . 
spin polarization of the outgoing wave is always larger than spin polarization of the incident wave. In this regime, peaks in the outgoing spin polarization coincide with peaks of the transmission and tend to have strongly spin polarized signals. So in this regime spin polarization has been amplified for the outgoing wave especially for the angle of incidence that transmission shows peaks. In the intermediate regime increasing $\bar{v}_{\mathrm{B}}$ results in increasing the spin polarization of the incident wave and starts to be comparable with spin polarization of the outgoing wave for some angles. Finally for the case of strong Rashba SOC strength, spin polarization of the incident waves is always bigger than the spin polarization of the outgoing wave. Further increasing of $\bar{v}_{\mathrm{B}}$ in this regime increases the spin polarization of the outgoing wave for the angle of incidence around $\phi=0$, in which the transmission probability is reaching its maximum value of 2 . In this regime, transmission peaks occur where there is mostly zero spin polarization for out going waves, so mostly transmission resonances are associated with spin unpolarized currents.

\subsection{N-P JUNCTION WITH SO-SO INTERFACES}

Here we consider a n-p junction in the presence of Rashba SOC. The wave functions for different regions are given by:

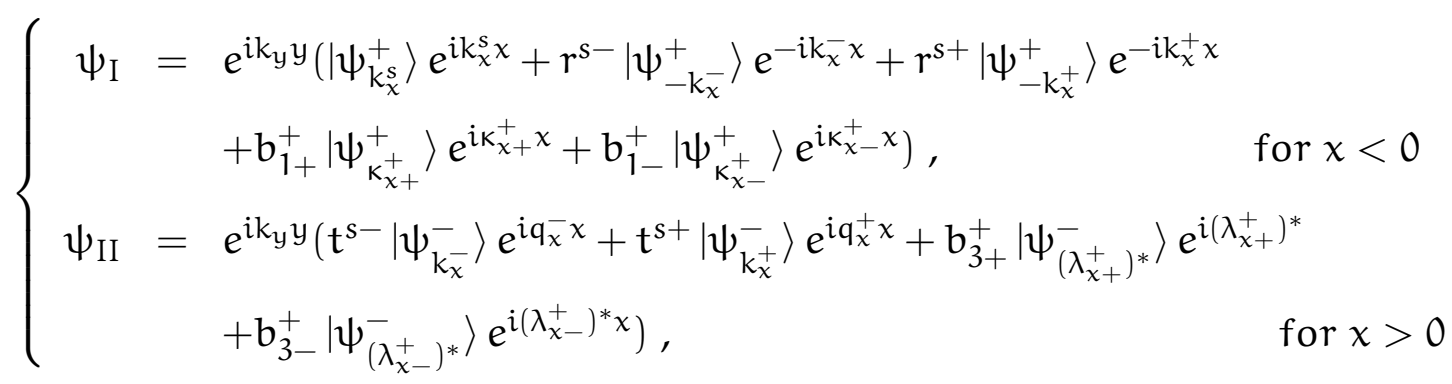

where $k_{x}^{s}$ and $q_{x}^{s}$ is the wave vector for the propagating wave with spin chirality $s$ for the incident region $(V=0)$ and the barrier $(V \neq 0)$ respectively. The sign of the wave vector inside the barrier is given by $\operatorname{sgn}\left(q_{x}^{s}\right)=\operatorname{sgn}\left(E_{f}-V\right) . \kappa_{x \pm}$ and $\lambda_{x \pm}$ are the wave vectors for the evanescent modes and the imaginary part of $\kappa_{x \pm}\left(\lambda_{x_{ \pm}}\right)$ must be negative (positive) in order to have a well defined wave function in the 

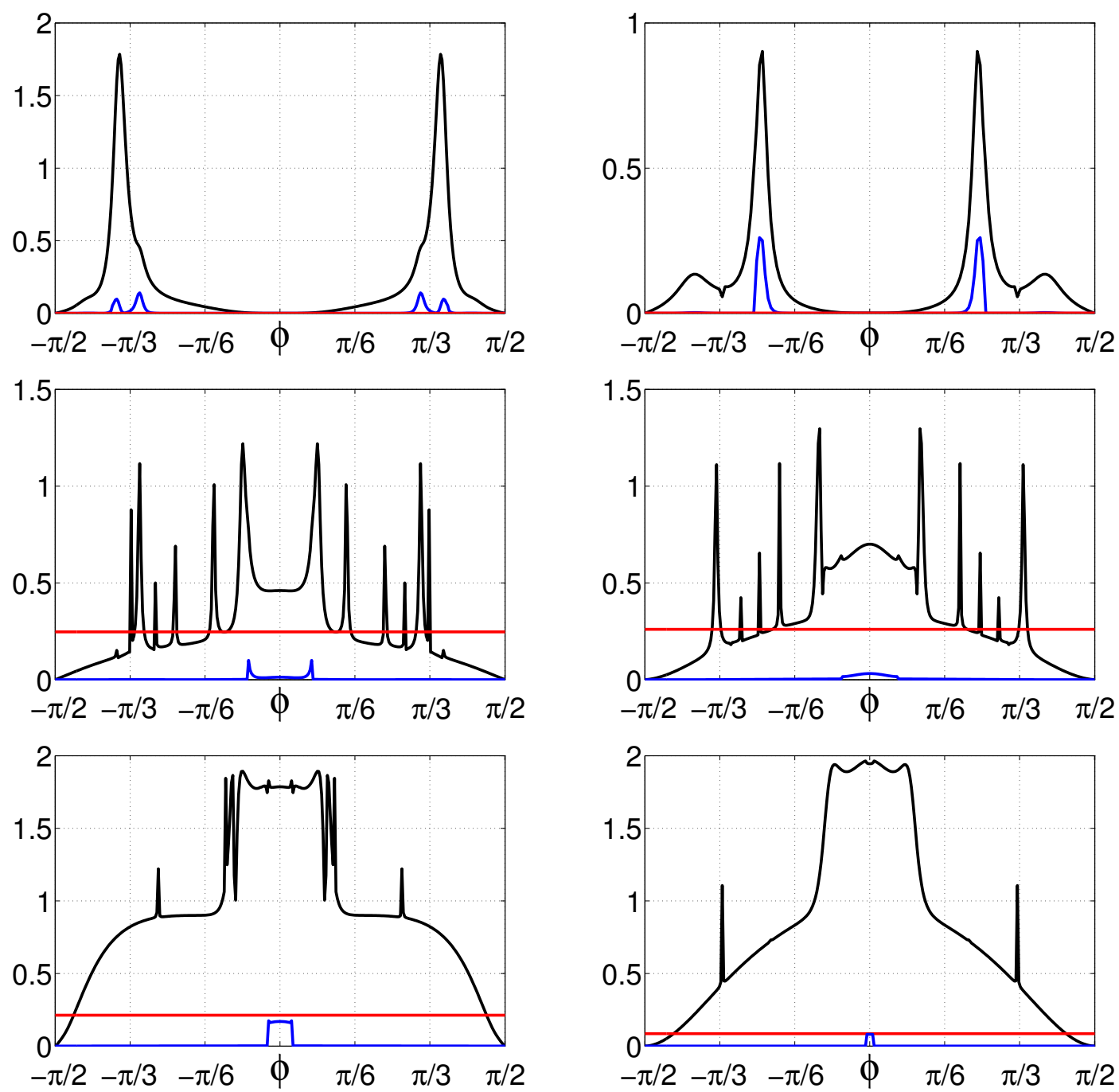

Figure 46: Angle resolved transmission probability $\mathrm{T}_{\text {total }}$ (black line) and spin polarization $\left\langle\mathrm{S}^{2}\right\rangle$ for the incident (red line) and transmitted electrons (blue line) in a n-p-n junction with width $100 \mathrm{~nm}$, height $100 \mathrm{meV}$ and Fermi energy $E_{f}=17$ meV with different Rashba SOC strength: $\bar{v}_{\mathrm{B}}=0.005$ (top left), $\bar{v}_{\mathrm{B}}=0.01$ (top right), $\bar{v}_{\mathrm{B}}=0.09$ (middle left), $\bar{v}_{\mathrm{B}}=0.1$ (middle right), $\bar{v}_{\mathrm{B}}=0.2$ (bottom left) and $\bar{v}_{\mathrm{B}}=0.5$ (bottom right) . 
incident region (barrier). The complex amplitudes $r^{s s^{\prime}}$ and $t^{s s^{\prime}}$ are the reflection and and transmission coefficients respectively. These coefficients are obtained by matching the wave function

$$
\left.\psi_{\mathrm{I}}\right|_{x=0}=\left.\psi_{\mathrm{II}}\right|_{x=0}
$$

and probability flux

$$
\left.\hat{v}_{x} \psi_{\mathrm{I}}\right|_{x=0}=\left.\hat{v}_{x} \psi_{\mathrm{II}}\right|_{x=0}
$$

at the interface of the junction, where the velocity operator in $x$-direction $\hat{v}_{x}$ is given by Eq. 265 .

\subsubsection{Comment on the Klein paradox for a pn junction in multilayer graphene}

In their paper, Duppen and Peeters (2013) studied the angle resolved transmission probability in a $n-p$ junction for multilayer graphene. They showed that multilayer graphene can have a transmission probability of one for some special angles (see Fig. 47). They used pseudospin conservation at the interface of the two regions to support their results. They mentioned that for some specific angles, the Hamiltonian of multilayer graphene is proportional to $\sigma_{x}$ or $\sigma_{y}$. Hence, Hamiltonian commutes with $\sigma_{x}$ or $\sigma_{y}$ which makes these observables representing pseudospin direction in $x$ and $y$ constants of motion. So they are expected to conserve pseudospin at these special angles, and if the reflected angle had opposite pseudospin direction, reflection would be forbidden and the electron would tunnel through the barrier with transmission probability one. For example they showed that for BLG, the Hamiltonian can be written as $\mathrm{H}_{\mathrm{BLG}}=$ $k^{2}\left(\cos \left(2 \phi_{k}\right) \sigma_{x}+\sin \left(2 \phi_{k}\right) \sigma_{y}\right)$ where $\phi_{k}=\arctan \left(k_{y} / k_{x}\right)$ and for angles $\phi_{k}=$ $\pm \pi / 4$, the sine in the Hamiltonian becomes zero. So for these angles $\left[\mathrm{H}_{\mathrm{BLG}}, \sigma_{\mathrm{x}}\right]=$ 0 , which makes $\sigma_{x}$ a conserved quantity.

We believe that they have made several mistakes in their calculations and physical interpretation. For instance, in their numerical calculation in order to have the same results, one needs to choose an evanescent wave function which goes 


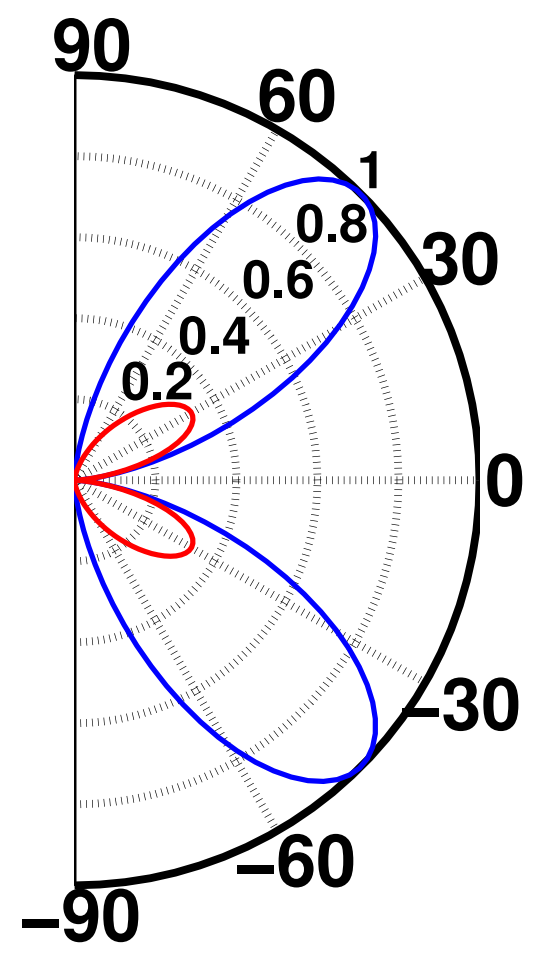

Figure 47: Transmission probability of a pn junction when $\mathrm{V}=2 \mathrm{E}_{\mathrm{F}}$. The blue curve shows the result found by Duppen and Peeters (2013) which indicates that when the angle of incidence is 45 degrees, the pn junction is transparent and transmission probability becomes one. The red curve shows the result found by our calculations where there is no perfect transition in a pn graphene junction.

to infinity for large values of $x$ in the $p$ junction, which is physically unrealistic. Regarding the physical interpretation of their results, one can simply show that the Hamiltonian is not the same for an angle of incidence with $\phi \neq 0$ and its reflection $(\pi-\phi)$. This feature implies that we can not consider conservation of pseudospin at these angles. Finally, it is well understood that the refraction angle for monolayer and bilayer graphene $n-p$ junction when $V>E_{f}$ is negative (Low et al., 2009; Cheianov et al., 2007a). This comes from the fact that in the $p$ region, holes in the valance band are responsible for carrying the current. These holes despite having a group velocity in the $x$ direction has wave vectors with negative values. This fact is missing in their interpretation as one can notice that in figure 


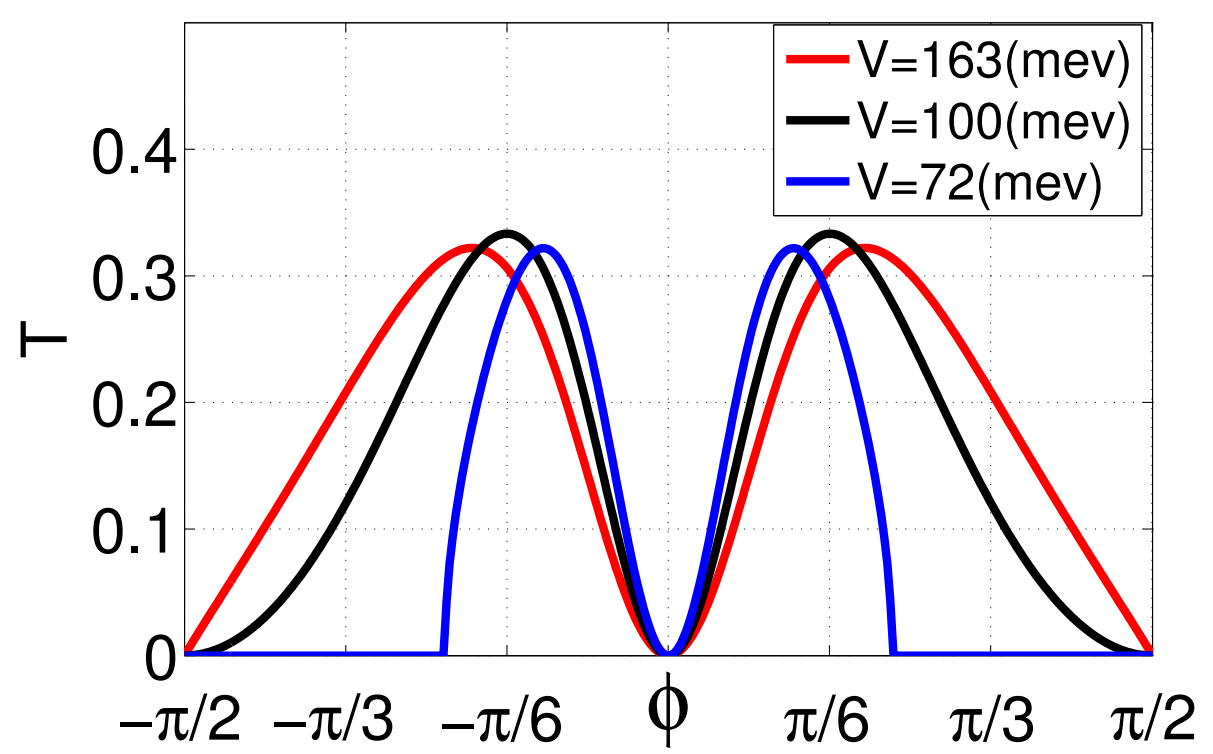

Figure 48: Transmission probability of a pn junction with different barrier height versus angle of incidence for electrons having Fermi energy $E_{f}=50 \mathrm{meV}$.

(3) of Duppen and Peeters (2013), the electrons with positive momentum tunnels into the states with positive wave vector values, which can not be correct.

\subsubsection{Numerical results for $n p$ junction}

In Fig. 48, the angle resolved transmission probability of a BLG p-n junction with Fermi energy $E_{f}=50 \mathrm{meV}$ for different heights of the barrier is shown. Here we have not considered the effect of Rashba SOC $\left(\bar{v}_{\mathrm{B}}=0\right)$. This result agrees with the fact that the transmission probability for normal incidence is zero $(T(0)=0)$ regardless of the height of the barrier. The absence of transmission or having full back scattering for normal incidence, can be explained using the chiral nature of the quasiparticles in BLG.

Now we turn on the Rashba SOC effect to see how it influences the transmission probability. In Fig. 49 the angle resolved transmission probability of a p-n junction with fermi energy $E_{f}=50 \mathrm{meV}$ and barrier height $V=100 \mathrm{meV}$ for different values of $\bar{v}_{\mathrm{B}}$ is shown. It can be understood from these results that as long as 

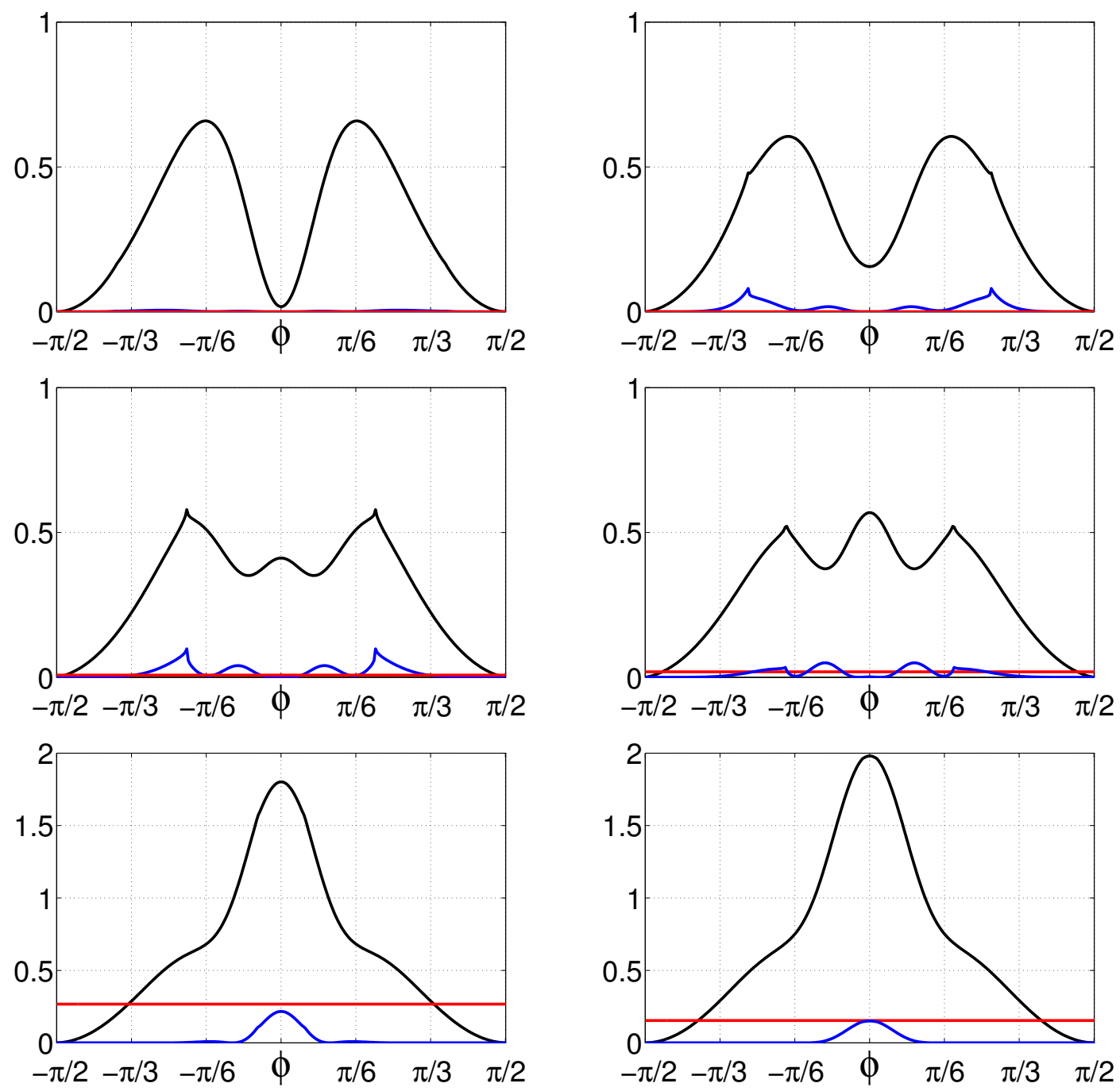

Figure 49: Angle resolved transmission probability $\mathrm{T}_{\text {total }}$ (black line) and spin polarization $\left\langle\mathrm{S}^{2}\right\rangle$ for the incident (red line) and transmitted electrons (blue line) in a n-p junction with height $100 \mathrm{meV}$ and Fermi energy $E_{f}=50 \mathrm{meV}$ with different Rashba SOC strength: $\bar{v}_{\mathrm{B}}=0.01$ (top left), $\bar{v}_{\mathrm{B}}=0.03$ (top right), $\bar{v}_{\mathrm{B}}=0.05$ (middle left), $\bar{v}_{\mathrm{B}}=0.06$ (middle right), $\bar{v}_{\mathrm{B}}=0.2$ (bottom left) and $\bar{v}_{\mathrm{B}}=0.5$ (bottom right). 
we are in the weak Rashba SOC regime $\left(4 \bar{k}^{2}>>\bar{v}_{\mathrm{B}}^{2}\right)$, the transmission probability is not very different from the case without Rashba SOC. Increasing the Rashba SOC strength results in having a non-zero normal transmission. Also, it is worth mentioning that in this regime, the spin polarization of the incident electrons are always smaller than the spin polarization of the transmitted electrons. In this regime the spin polarization of the transmitted electrons increases by increasing the strength of the Rashba SOC (see top row in Fig. 49). For very large $\bar{v}_{\mathrm{B}}$, the system is in the strong Rashba SOC regime $\left(4 \bar{k}^{2}<<\bar{v}_{\mathrm{B}}^{2}\right)$. In this regime by increasing $\bar{v}_{\mathrm{B}}$, the transmission probability becomes more similar to what we have in the case of a monolayer. The reason is obvious as by increasing $\bar{v}_{\mathrm{B}}$ the linear term in Eq. 253 becomes dominant. In this regime, the spin polarization of the incident electrons is always larger than the spin polarization of the transmitted electrons. By increasing the Rashba SOC strength, the spin polarization around $\phi=0$ starts to grow until it finally reaches to the spin polarization of the incident electrons (see bottom row in Fig. 49). In the intermediate regime, the spin polarization of the incident waves begins to rise by increasing $\bar{v}_{\mathrm{B}}$. On the other hand, the spin polarization of the transmitted waves starts to decrease (see middle row in Fig. 49)

\subsection{N-P-N JUNCTION WITH N-SO-N INTERFACES}

In this section we investigate electron spin tunneling through a Rashba barrier. We assume that Rashba SOC is absent everywhere in the junction except for the barrier. We solve the tunneling problem by considering the wave function in different regions as follows: 


$$
\begin{aligned}
\psi_{\mathrm{I}}= & \left|\psi_{k_{x}^{s}}^{+}\right\rangle e^{i k_{x}^{s} x}+r^{\uparrow}\left|\psi_{-k_{x}^{\uparrow}}^{+}\right\rangle e^{-i k_{x}^{\uparrow} x}+r^{\downarrow}\left|\psi_{-k_{x}^{\downarrow}}^{+}\right\rangle e^{-i k_{x}^{\downarrow} x} \\
& +b_{1}^{\downarrow}\left|\psi_{k_{x}^{\downarrow}}^{+}\right\rangle e^{k_{x}^{\downarrow} x}+b_{1}^{\uparrow}\left|\psi_{k_{x}^{+}}^{+}\right\rangle e^{k_{x}^{\uparrow} x} \\
\psi_{\text {II }}= & a_{2}^{-}\left|\psi_{q_{x}^{-}}^{-}\right\rangle e^{i q_{x}^{-} x}+a_{2}^{+}\left|\psi_{q_{x}^{+}}^{-}\right\rangle e^{i q_{x}^{+} x}+b_{2}^{-}\left|\psi_{-q_{x}^{-}}^{-}\right\rangle e^{-i q_{x}^{-} x} \\
& +b_{2}^{+}\left|\psi_{-q_{x}^{+}}^{-}\right\rangle e^{-i q_{x}^{+} x}+c_{2-}^{+}\left|\psi_{\lambda_{x-}^{+}}^{-}\right\rangle e^{i \lambda_{x}^{+} x}+c_{2+}^{+}\left|\psi_{\lambda_{x+}^{+}}^{-}\right\rangle e^{i \lambda_{x}^{+} x} \\
& +d_{2-}^{+}\left|\psi_{\left(\lambda_{x}^{-}\right)^{*}}^{-}\right\rangle e^{i\left(\lambda_{x-}^{+}\right)^{*} x}+d_{2+}^{+}\left|\psi_{\left(\lambda_{x+}^{+}\right)^{*}}^{-}\right\rangle e^{i\left(\lambda_{x+}^{+}\right)^{*} x} \\
\psi_{\text {III }}= & t^{\uparrow}\left|\psi_{k_{x}^{\uparrow}}^{+}\right\rangle e^{i k_{x}^{\uparrow} x}+t^{\downarrow}\left|\psi_{k_{x}^{+}}^{+}\right\rangle e^{-i k_{x}^{\downarrow} x}+b_{3}^{\uparrow}\left|\psi_{-k_{x}^{\uparrow}}^{+}\right\rangle e^{-k_{x}^{\uparrow}} \\
& +b_{3}^{\downarrow}\left|\psi_{-k_{x}^{\downarrow}}^{+}\right\rangle e^{-k_{x}^{\downarrow} x}
\end{aligned}
$$

where $k_{x}^{2}+k_{y}^{2}=\frac{2 m|E|}{\hbar^{2}}$ and $k_{x}^{2}-k_{y}^{2}=\frac{2 m|E|}{\hbar^{2}}$ are wave vectors for the propagating and evanescent waves outside the barrier respectively, and $q_{x}^{-}, q_{x}^{+}$and $\lambda_{x \pm}^{+}$are obtained by solving Eq. 255 for the Rashba barrier $\left(\bar{v}_{B} \neq 0\right)$. Again by applying the continuity of the wave function Eq. 269 and probability current Eq. 270 we can find the unknown coefficients of the wave functions in Eq. 271 . For calculating the angle resolved transmission and comparing them with the results reported by Katsnelson et al. (2006), we use the same structure parameters, that is we consider the height of the barrier as $\mathrm{V}=0.0043 \mathrm{E}_{0}(50 \mathrm{meV})$, the width of the barrier as $W=406.33 a(100 \mathrm{~nm}$ ) and the energy of the incident electron with $E_{f}=0.00146 E_{0}(17 \mathrm{meV})$ where $E_{0}=\frac{\hbar^{2}}{2 M^{2}}$ and $a=2.46 \AA$ is the graphene lattice parameter. We choose the quantization axis in $z$ direction and consider that the spin of the incident wave is up. Hence, the transmitted waves corresponding to up and down spin can be shown by $\mathrm{T}_{\uparrow \uparrow}$ and $\mathrm{T}_{\uparrow \downarrow}$ respectively.

In Fig. 50 the angle resolved transmission probability $T_{\text {total }}$ and spin polarization $\left\langle S_{z}\right\rangle$ for different values of the Rashba SOC strength are shown. In the left column of Fig. 50, the transmission probability for incidence wave with up spin is shown. The blue curve $T_{\uparrow \downarrow}$ shows the transmission of the outgoing electrons with spin down when the incident electrons have spin up, whereas rthe ed curve $\mathrm{T}_{\uparrow \uparrow}$ is the transmission probability for outgoing electrons with spin up when the 

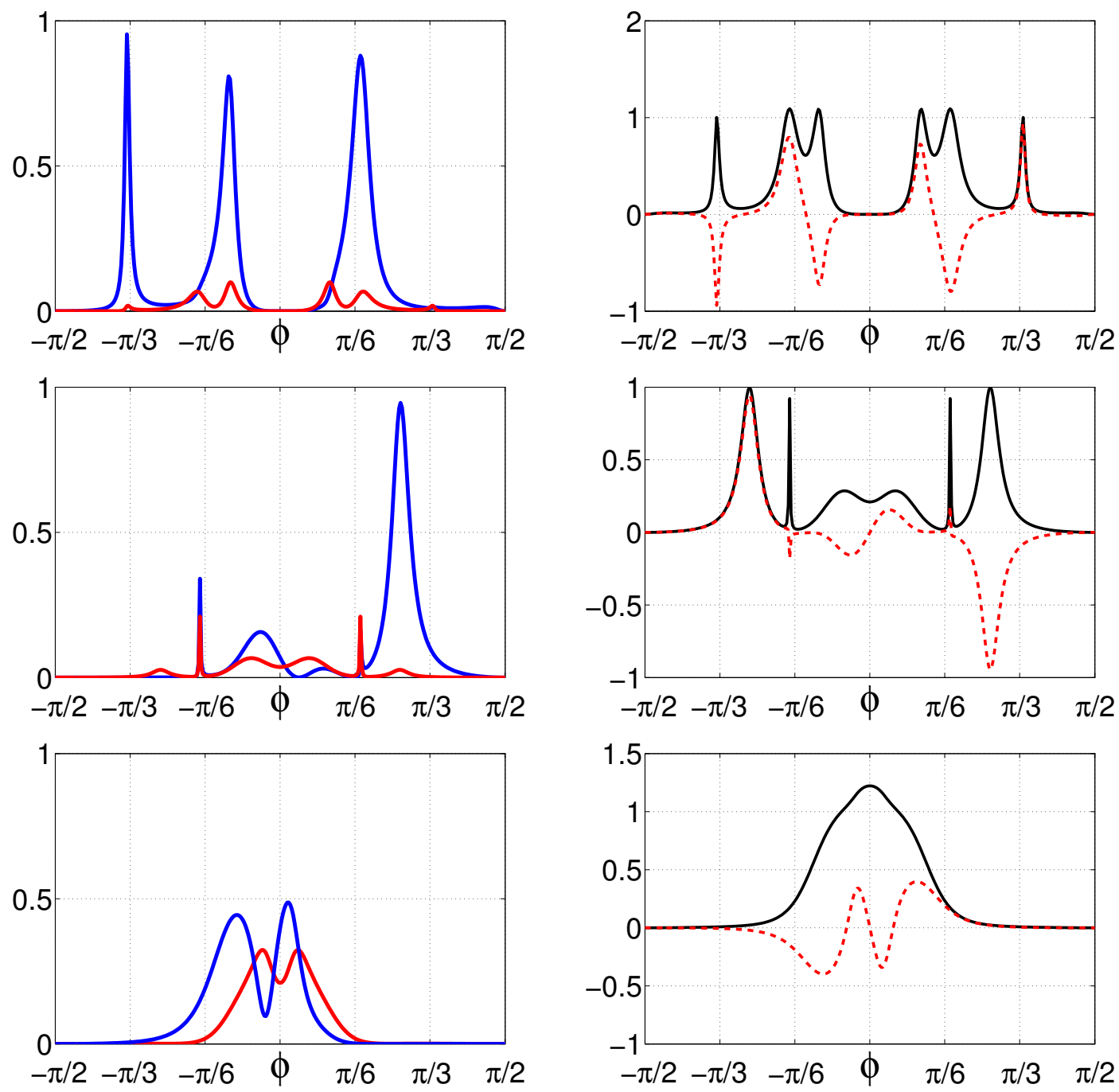

Figure 50: Right column: angle resolved total transmission probability $\mathrm{T}_{\text {total }}$ (black line) and spin polarization in $z$ direction $\left\langle\mathrm{S}_{z}\right\rangle$ (dashed red line). Left column: transmission probability of outgoing electrons with spin up (down) when the spin of the incident electrons is up $\left(T_{\uparrow \uparrow}\left(T_{\uparrow \downarrow}\right)\right)$ for a Rashba SOC strength of $\bar{v}_{\mathrm{B}}=0.005$ (first row), $\bar{v}_{\mathrm{B}}=0.01$ (second row), $\bar{v}_{\mathrm{B}}=0.05$ (third row). 
incident electrons have up spin. The transmission probability for the incident waves with down spin has the symmetry

$$
\begin{aligned}
& \mathrm{T}_{\uparrow \uparrow}(\phi)=\mathrm{T}_{\downarrow \downarrow}(\phi), \\
& \mathrm{T}_{\uparrow \downarrow}(\phi)=\mathrm{T}_{\downarrow \uparrow}(-\phi) .
\end{aligned}
$$

As we see in the left column of Fig. 50, for weak Rashba SOC strength, the Rashba barrier tends to inverse the spin of the incoming electrons. In the right column of Fig. 50 we show the total transmission probability $\left(T_{\text {total }}=\sum_{s, s^{\prime}=\uparrow \downarrow} T_{s s,}\right)$ and expectation value of spin in $z$ direction $\left(\left\langle S_{z}\right\rangle\right)$. Here we have assumed that the incoming wave is unpolarized $\left(\left\langle S_{z}\right\rangle=0\right)$ which means it consists equally of electrons with up and down spin. However the spin polarization of the outgoing electrons regarding their angle of incidence can be finite $\left\langle S_{z}\right\rangle_{\phi} \neq 0$. Peaks of spin polarization for weak Rashba SOC strength coincide with the peaks of the transmission probability. The spin polarization $\left\langle S_{z}\right\rangle$ changes its sign between consecutive peaks. In weak Rashba SOC regime, there are peaks in the transmission probability with $\left\langle S_{z}\right\rangle \simeq 1$. However in strong Rashba sOC regime $\bar{v}_{\mathrm{B}}=0.05$, the transmission probability has only one peak around $\phi=0$. This feature happens due to the dominance of the linear Rashba term Eq. 253. The spin of the out going electrons do not have any component in the $x y$ direction $\left\langle S_{x, y}\right\rangle=0$. Notice

that $\int_{-\pi / 2}^{\pi / 2}\left\langle S_{z}\right\rangle d \phi=0$, so the outgoing current in not polarized. However, the Rashba barrier in bilayer graphene npn junctions can be used to invert the spin of a polarized current.

\subsection{SUMMARY AND CONCLUSION}

Here in this section we investigated the effect of Rashba SOC in transport properties of a BLG system by studying chiral tunneling through npn and np junctions. We showed that introducing Rashba SOC in BLG can affect the perfect transmission seen in BLG. For sufficiently large Rashba SOC strengths normal transmission which is forbidden in BLG becomes finite. As we increase the strength of the Rashba SOC, we can see perfect transmission for normal incidence electrons. We 
showed that in the weak Rashba SOC regime, the spin polarization of outgoing electrons have peaks which coincide with the peaks of the transmission probability. For the weak Rashba SOC regime, the spin polarization of the outgoing electrons are always larger than the spin polarization of the incidence ones. We reported our results for the npn junction with Rashba barrier. We showed that Rashba barrier can inverse the spin of the incidence electrons. 
SPIN SUSCEPTIBILITY OF TWO-DIMENSIONAL

TRANSITION METAL DICHALCOGENIDES

\subsection{INTRODUCTION AND MOTIVATION}

Recent theoretical studies on $\mathrm{MoS}_{2}$ have focused on the many-particle and collective response properties of its charge carriers. Plasmon dispersions and static screening have been investigated within the random phase approximation (Scholz et al., 2013). Other works (Lu et al., 2013; Song and Dery, 2013; Ochoa et al., 2013; Ochoa and Roldán, 2013; Wang and $\mathrm{Wu}$; $\mathrm{Yu}$ and $\mathrm{Wu}$ ) have discussed the various spin-relaxation processes that can occur in $\mathrm{MoS}_{2}$. Furthermore, the carriermediated exchange interaction between localized magnetic impurities has been calculated (Parhizgar et al., 2013) within the framework of the RKKY mechanism (Ruderman and Kittel, 1954; Kasuya, 1956; Yosida, 1957) and using first-principle methods (Cheng et al., 2013; Mishra et al., 2013). A recent study (Dolui et al., 2013) has systematically explored realistic strategies for achieving $n$-type and p-type doping in monolayer $\mathrm{MoS}_{2}$.

Analytical results for the wave-vector-dependent spin susceptibility (Moriya, 1985; Yosida, 1996) $\chi_{i j}(\mathbf{q})$ are obtained based on the $\mathbf{k} \cdot \mathbf{p}$ model-Hamiltonian descriptions (Xiao et al., 2012; Kormányos et al., 2013; Rostami et al., 2013). Physical consequences are discussed and illustrated using band-structure parameters for $\mathrm{MoS}_{2}$.

Here in this chapter, we reveal interesting features are exhibited by carriermediated exchange interactions between local magnetic moments and Zeeman spin splitting as encoded in the electronic $\mathrm{g}$-factor. The hole-doped material turns out to have particularly rich spin properties, whereas the electron-doped case shows behavior quite similar to that of ordinary $2 \mathrm{D}$ electron systems. Nevertheless, from a conceptual point of view, consideration of the electron-doped mate- 
rial is useful because it serves as an instructive testbed for understanding the interplay between extrinsic and intrinsic contributions to the spin response, where the former (latter) result from filled states in the conduction (valence) band.

Thus the spin-response properties of monolayer transition metal dichalcogenides constitute an intriguing intermediate behavior between that exhibited by graphene and ordinary $2 \mathrm{D}$ electron systems realized in semiconductor heterostructures. Besides adding to the basic understanding of a new material class, our results also suggest practical ways for electronic manipulation of its spin structure.

\subsection{DETAILS OF OUR THEORETICAL APPROACH}

\subsubsection{Model-Hamiltonian description}

As our formal basis for the calculation of the spin susceptibility, we adopt the low-energy effective Hamiltonian for monolayer transition metal dichalcogenides derived in Ref. (Xiao et al., 2012) (see also Refs. (Kormányos et al., 2013; Rostami et al., 2013)). To lowest order in the in-plane wave vector $\mathbf{k}=\left(k_{x}, k_{y}\right)$, it reads

$$
H_{0}^{\tau}=\operatorname{at}\left(\tau k_{x} \hat{\sigma}_{x}+k_{y} \hat{\sigma}_{y}\right) \otimes 1+\frac{\Delta}{2} \hat{\sigma}_{z} \otimes 1-\frac{\lambda \tau}{2}\left(\hat{\sigma}_{z}-1\right) \otimes \hat{s}_{z}
$$

The valley index $\tau= \pm 1$ distinguishes electronic excitations at the two nonequivalent high-symmetry points $\mathbf{K}$ and $\mathbf{K}^{\prime} \equiv-\mathbf{K}$ in the Brillouin zone. The symbol a denotes the lattice constant, $t$ is the nearest-neighbor hopping matrix element, $\Delta$ is the fundamental energy gap between conduction and valence bands, and $2 \lambda$ is a measure of the material's intrinsic spin-orbit coupling strength. The Pauli matrices $\hat{\sigma}_{x, y, z}$ act in the space of basis functions for the conduction and valenceband states at the $\mathbf{K}$ and $\mathbf{K}^{\prime}$ points. In contrast, $\hat{\mathbf{s}}_{z}$ is the diagonal Pauli matrix associated with the charge carriers' real spin. We neglect the recently discussed (Kormányos et al., 2013; Rostami et al., 2013) corrections to effective band masses and trigonal warping, which only give rise to small quantitative corrections to the spin susceptibility. For the case of $\mathrm{MoS}_{2}$, values of the relevant parameters are (Xiao et al., 2012) $a=3.193 \AA, t=1.1 \mathrm{eV}, \Delta=1.66 \mathrm{eV}$, and $2 \lambda=0.15 \mathrm{eV}$. These 
values have been used in our calculations whose results are plotted in the figures of this chapter.

The term proportional to $\lambda$ in Eq. (273) breaks the spin-rotational invariance in our system of interest; with eigenstates having their real spin quantized along the out-of-plane $(z)$ direction. In the following, we use a representation where the space of conduction (c) and valence (v) bands is combined with the real-spin space, and we will adopt the states $|\mathrm{c} \uparrow\rangle,|\mathrm{v} \uparrow\rangle,|\mathrm{c} \downarrow\rangle,|\mathrm{v} \downarrow\rangle$ from each individual valley as our basis. The generalized Pauli matrices for real spin are then given by $\hat{J}_{i}=\hat{J}_{i} \otimes \hat{\tau}_{0}$, with $\hat{\tau}_{0} \equiv 1_{2 \times 2}$ being the identity matrix in valley space, and

$$
\begin{aligned}
& \hat{J}_{x}=\left(\begin{array}{llll}
0 & 0 & 1 & 0 \\
0 & 0 & 0 & 1 \\
1 & 0 & 0 & 0 \\
0 & 1 & 0 & 0
\end{array}\right), \\
& \hat{J}_{y}=\left(\begin{array}{cccc}
0 & 0 & -i & 0 \\
0 & 0 & 0 & -i \\
i & 0 & 0 & 0 \\
0 & i & 0 & 0
\end{array}\right), \\
& \hat{J}_{z}=\left(\begin{array}{cccc}
1 & 0 & 0 & 0 \\
0 & 1 & 0 & 0 \\
0 & 0 & -1 & 0 \\
0 & 0 & 0 & -1
\end{array}\right) .
\end{aligned}
$$




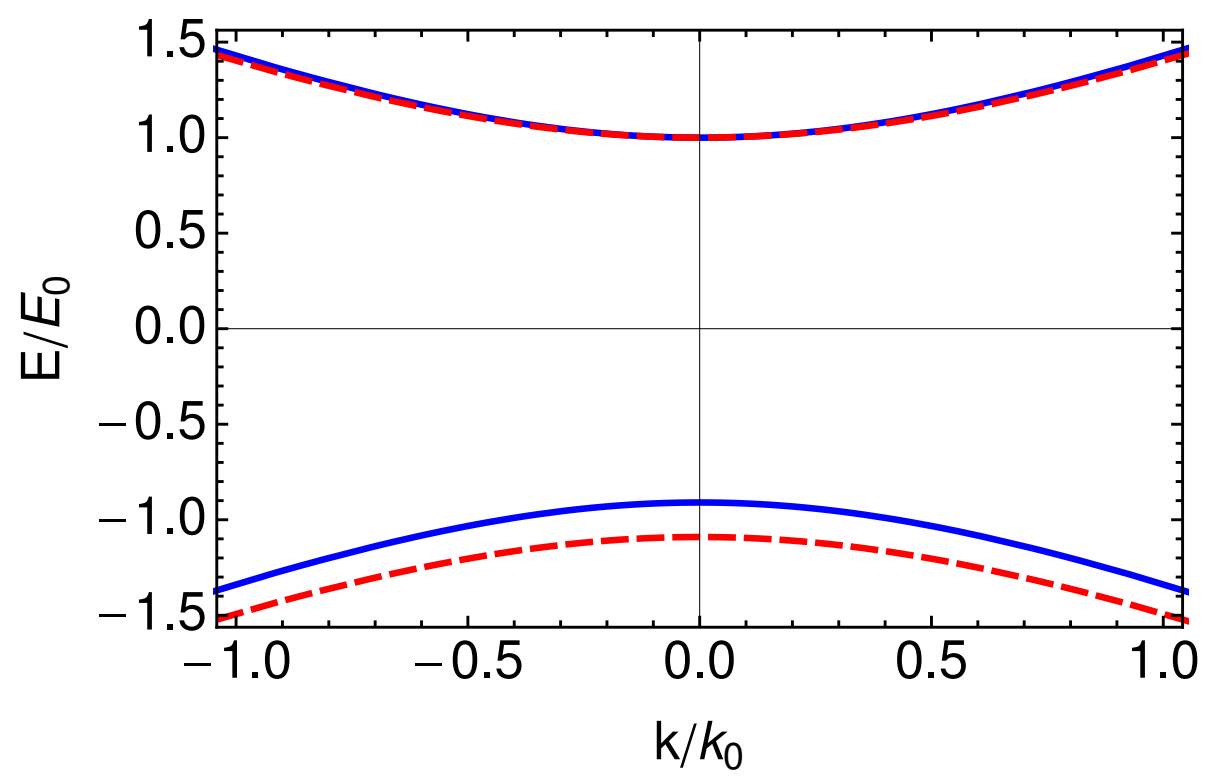

Figure 51: Spin-resolved band dispersions for monolayer $\operatorname{MoS}_{2}$ at the $\mathbf{K}$ point. Spin- $\uparrow(\downarrow)$ bands are shown as the blue solid (red dashed) curves. The unit scales for energy $E$ and wave vector $\mathbf{k}$ (measured from $\mathbf{K}$ ) are given in terms of bandstructure parameters as $E_{0} \equiv \Delta / 2$ and $k_{0} \equiv \Delta /(2 a t)$. Reversal of all spin labels yields the corresponding band dispersions at the $\mathbf{K}^{\prime}(\equiv-\mathbf{K})$ point.

It is instructive to express also the model Hamiltonian given in Eq. (273) as a matrix corresponding to our chosen representation. Using polar coordinates $\mathbf{k}=$ $(k, \theta)$ for the in-plane wave vector, we find

$$
H_{0}^{\tau}=\left(\begin{array}{cccc}
\frac{\Delta}{2} & a t \tau k e^{-i \theta} & 0 & 0 \\
a t \tau k e^{i \theta} & -\frac{\Delta}{2}+\lambda \tau & 0 & 0 \\
0 & 0 & \frac{\Delta}{2} & a t \tau k e^{-i \theta} \\
0 & 0 & a^{i t} k e^{i \theta} & -\frac{\Delta}{2}-\lambda \tau
\end{array}\right) .
$$

The eigenenergies and eigenstates of the Hamiltonian $\mathrm{H}_{0}^{\tau}$ are straightforwardly obtained as

$$
E_{\mathbf{k} \alpha}^{(\tau, s)}=\frac{E_{0}}{2}\left(s \tau \bar{\lambda}+\alpha \sqrt{4 \bar{k}^{2}+m_{s \tau}^{2}}\right)
$$


and

$$
\psi_{\mathbf{k} \alpha}^{(\tau, s)}=\frac{1}{\sqrt{2}}\left(\begin{array}{c}
\alpha\left[1+\frac{\alpha m_{s \tau}}{\sqrt{4 \bar{k}^{2}+m_{s \tau}^{2}}}\right]^{\frac{1}{2}} \\
\tau\left[1-\frac{\alpha m_{s \tau}}{\sqrt{4 \bar{k}^{2}+m_{s \tau}^{2}}}\right]^{\frac{1}{2}} e^{-i \tau \theta}
\end{array}\right) \otimes|s\rangle,
$$

respectively, where $\alpha=1(-1)$ for conduction electrons (valence-band holes), $s= \pm 1$ labels the eigenstates of $s_{z}$, and $m_{ \pm} \equiv 2 \mp \bar{\lambda}$. We introduced dimensionless quantities $\bar{k}=k / k_{0}, \bar{\lambda} \equiv \lambda / E_{0}$, with unit scales for energy and wave vector given by $E_{0} \equiv \Delta / 2$ and $k_{0} \equiv \Delta /(2 a t)$, respectively. In the limit $\lambda \rightarrow 0$, the effective Hamiltonian (273) is equivalent to a Dirac model with effective speed of light $c_{\text {eff }} \equiv a t / \hbar$ and effective rest mass $M_{\text {eff }} \equiv \hbar^{2} \Delta /\left(2 a^{2} t^{2}\right)$. Hence, the scales $E_{0}$ and $k_{0}$ can be associated with an effective rest energy $M_{\text {eff }} c_{\text {eff }}^{2}$ and inverse Compton wave length $M_{\text {eff }} c_{\text {eff }} / \hbar$, respectively. Later on we choose $\chi_{0}=2 k_{0}^{2} /\left(\pi \mathrm{E}_{0}\right) \equiv 2 \mathrm{M}_{\mathrm{eff}} /\left(\pi \hbar^{2}\right)$ as the unit for the spin-susceptibility tensor, as it corresponds to the density of states for a $2 \mathrm{D}$ system of free electrons with effective mass $M_{\text {eff }}$ and four-fold flavor degeneracy. Figure 51 shows the electron and hole band dispersions obtained for $\mathrm{MoS}_{2}$. Note that SOC gives rise to an energy splitting for the hole (valence-band) excitations that is finite $(\equiv 2 \lambda)$ even at the band edge. For a non-zero wave vector, the inter-band coupling induces a spin splitting also for conduction electrons, but its magnitude is suppressed because of the relatively large band gap.

When the Fermi energy $E_{F}$ is above (below) the conduction-band (valenceband) edge, the system is electron-doped (hole-doped). The Fermi wave vectors for electronic excitations associated with the spin-split bands in each valley are then given by $k_{\mathrm{F}}^{(s \tau)}$, with

$$
k_{\mathrm{F}}^{( \pm)}=k_{0} \sqrt{\left[\left(E_{F} / E_{0}\right)-1\right]\left[\left(E_{F} / E_{0}\right)+1 \mp \bar{\lambda}\right]} .
$$

The total sheet density $n$ of charge carriers can be related to the Fermi wave vectors via

$$
\mathrm{n}=\frac{1}{4 \pi} \sum_{\tau, \mathrm{s}}\left(\mathrm{k}_{\mathrm{F}}^{(s \tau)}\right)^{2} \equiv \frac{\mathrm{g}_{v}}{4 \pi}\left[\left(\mathrm{k}_{\mathrm{F}}^{(+)}\right)^{2}+\left(\mathrm{k}_{\mathrm{F}}^{(-)}\right)^{2}\right],
$$

where $g_{v}=2$ is the degeneracy factor associated with the valley degree of freedom. In the following, it will be useful to also define a density-related average 
Fermi wave number $k_{F}$ such that $n=g_{v} g_{s} k_{F}^{2} /(4 \pi)$, with real-spin degeneracy factor $g_{s}=2$. Obviously we have

$$
k_{F}=\left\{\begin{array}{cl}
k_{0}\left[\left(\frac{E_{F}}{E_{0}}\right)^{2}-1\right]^{\frac{1}{2}} & E_{F}>E_{0} \text { or } E_{F}<-E_{0}-\lambda \\
k_{F}^{(+)} & -E_{0}+\lambda>E_{F}>-E_{0}-\lambda
\end{array} .\right.
$$

\subsubsection{Spin susceptibility for a multi-band system}

The influence of spin-dependent external stimuli on a many-particle system can be quite generally discussed, within linear-response theory, in terms of the spin susceptibility given by (Giuliani and Vignale, 2005)

$$
\chi_{i j}\left(\mathbf{r}-\mathbf{r}^{\prime}\right)=-\frac{i}{\hbar} \int_{0}^{\infty} d t e^{-\eta t}\left\langle\left[S_{i}(\mathbf{r}, t), S_{j}\left(\mathbf{r}^{\prime}, 0\right)\right]\right\rangle .
$$

Here $S_{j}(\mathbf{r})$ denotes a general Cartesian component of the spin-density operator (we measure spin in units of $\hbar$ ), and $\mathbf{r}$ is the position vector in the $x y$-plane. We can express $S_{j}(\mathbf{r})$ in terms of the second-quantized particle creation and annihilation operators $\Psi^{\dagger}, \Psi$ and the spin matrices $\hat{\partial}_{j}$ as $S_{j}(\mathbf{r})=\Psi^{\dagger}(\mathbf{r}) \hat{\partial}_{j} \Psi(\mathbf{r})$. As particle excitations are generally superpositions of contributions from the individual valleys, we represent the particle operator as a spinor, $\Psi(\mathbf{r})=\left(\Psi^{(+)}(\mathbf{r}), \Psi^{(-)}(\mathbf{r})\right)$. In

terms of energy eigenstates and their annihilation operators $c_{\mathbf{k} \alpha}^{(\tau, s)}$, the contributions for each valley can be expressed as

$$
\Psi^{\tau}(\mathbf{r})=\sum_{s, \alpha} \int \frac{d^{2} k}{(2 \pi)^{2}} e^{i(\mathbf{k}+\tau \mathbf{K}) \mathbf{r}} \psi_{\mathbf{k} \alpha}^{(\tau, s)} c_{\mathbf{k} \alpha}^{(\tau, s)}
$$

With these definitions, we explicitly write the spin operator as

$$
S_{i}(\mathbf{r})=\sum_{\alpha, \beta} \int \frac{d^{2} k}{(2 \pi)^{2}} \int \frac{d^{2} k^{\prime}}{(2 \pi)^{2}} e^{i\left(\mathbf{k}^{\prime}-\mathbf{k}\right) \mathbf{r}}\left(\psi_{\mathbf{k} \alpha}^{\dagger} \hat{J}_{i} \psi_{\mathbf{k}^{\prime} \beta}\right) c_{\mathbf{k} \alpha}^{\dagger} c_{\mathbf{k}^{\prime} \beta},
$$

where we have used a greek index to include the quantum numbers for sublattice, spin and valley. The time-dependent spin operator is given by $S_{\mathfrak{i}}(\mathbf{r}, \mathrm{t})=$ 
$e^{\frac{i}{\hbar} H_{0} t} S_{i}(\mathbf{r}) e^{-\frac{i}{\hbar} H_{0} t}=e^{i\left(E_{\mathbf{k}_{\alpha}}-E_{\mathbf{k}^{\prime} \beta}\right) \frac{t}{\hbar}} S_{i}(\mathbf{r})$. The commutator under the integral in (281) is then given by

$$
\begin{aligned}
{\left[S_{i}(\mathbf{r}, \mathrm{t}), S_{j}\left(\mathbf{r}^{\prime}\right)\right]=} & \sum_{\alpha, \beta, \gamma, \delta} \int \frac{d^{2} k}{(2 \pi)^{2}} \int \frac{d^{2} k^{\prime}}{(2 \pi)^{2}} \int \frac{d^{2} k^{\prime \prime}}{(2 \pi)^{2}} \int \frac{d^{2} k^{\prime \prime \prime}}{(2 \pi)^{2}} e^{i\left(\mathbf{k}^{\prime}-\mathbf{k}\right) \mathbf{r}} \mathrm{e}^{i\left(\mathbf{k}^{\prime \prime \prime}-\mathbf{k}^{\prime \prime}\right) \mathbf{r}^{\prime}} \\
& \mathrm{e}^{i\left(E_{\mathbf{k} \alpha}-E_{\mathbf{k}^{\prime} \beta}\right) \frac{\mathrm{t}}{\hbar}}\left(\psi_{\mathbf{k} \alpha}^{\dagger} \hat{J}_{i} \psi_{\mathbf{k}^{\prime} \beta}\right)\left(\psi_{\mathbf{k}^{\prime \prime} \gamma}^{\dagger} \hat{J}_{j} \psi_{\mathbf{k}^{\prime \prime \prime} \delta}\right)\left[c_{\mathbf{k} \alpha}^{\dagger} c_{\mathbf{k}^{\prime} \beta}, c_{\mathbf{k}^{\prime \prime} \gamma}^{\dagger} c_{\mathbf{k}^{\prime \prime \prime} \delta}\right] .
\end{aligned}
$$

The commutator in (284) involving the creation and annihilation operators is evaluated to give $\left[S_{i}(\mathbf{r}, t), S_{j}\left(\mathbf{r}^{\prime}\right)\right](2 \pi)^{2} \delta^{(2)}\left(\mathbf{k}^{\prime}-\mathbf{k}^{\prime \prime}\right) \delta_{\beta \gamma} c_{\mathbf{k} \alpha}^{\dagger} c_{\mathbf{k}^{\prime \prime \prime} \delta}-(2 \pi)^{2} \delta^{(2)}\left(\mathbf{k}-\mathbf{k}^{\prime \prime \prime}\right) \delta_{\alpha \delta} c_{\mathbf{k}^{\prime \prime} \gamma}^{\dagger} c_{\mathbf{k}^{\prime} \beta}$. The equilibrium average of the same commutator gives

$$
\left\langle\left[S_{i}(\mathbf{r}, t), S_{j}\left(\mathbf{r}^{\prime}\right)\right]\right\rangle=(2 \pi)^{4} \delta_{\beta \gamma} \delta_{\alpha \delta} \delta^{(2)}\left(\mathbf{k}^{\prime}-\mathbf{k}^{\prime \prime}\right) \delta^{(2)}\left(\mathbf{k}-\mathbf{k}^{\prime \prime \prime}\right)\left[n_{\mathrm{F}}\left(E_{\mathbf{k} \alpha}\right)-n_{\mathrm{F}}\left(E_{\mathbf{k}^{\prime} \beta}\right)\right] .
$$

Performing the summations and trivial integrations yields

$$
\begin{aligned}
\left\langle\left[S_{i}(\mathbf{r}, t), S_{j}\left(\mathbf{r}^{\prime}\right)\right]\right\rangle= & \sum_{\alpha, \beta} \int \frac{d^{2} k}{(2 \pi)^{2}} \int \frac{d^{2} k^{\prime}}{(2 \pi)^{2}} e^{i\left(\mathbf{k}^{\prime}-\mathbf{k}\right)\left(\mathbf{r}-\mathbf{r}^{\prime}\right)} e^{i\left(E_{\mathbf{k} \alpha}-E_{\mathbf{k}^{\prime} \beta}\right) \frac{t}{\hbar}} \\
& \left(\psi_{\mathbf{k} \alpha}^{\dagger} \hat{J}_{i} \psi_{\mathbf{k}^{\prime} \beta}\right)\left(\psi_{\mathbf{k}^{\prime} \beta}^{\dagger} \hat{J}_{j} \psi_{\mathbf{k} \alpha}\right)\left[n_{F}\left(E_{\mathbf{k} \alpha}\right)-n_{F}\left(E_{\mathbf{k}^{\prime} \beta}\right)\right] .
\end{aligned}
$$

Making in (286) the variable transformations $\mathbf{k}^{\prime}=\mathbf{q}+\mathbf{k}$ and $\mathbf{R}=\mathbf{r}-\mathbf{r}^{\prime}$, and finally performing the time integration on the r.h.s. of (281) yields

$$
\chi_{i j}(\mathbf{R})=\int \frac{d^{2} q}{(2 \pi)^{2}} e^{i q R} \chi_{i j}(\mathbf{q})
$$

and

$$
\chi_{i j}(\mathbf{q})=\sum_{s, s^{\prime}, \tau} \sum_{\alpha, \beta} \int \frac{d^{2} k}{(2 \pi)^{2}} \mathscr{W}_{i j(\mathbf{k}, \mathbf{k}+\mathbf{q}, \alpha, \beta)}^{s, s^{\prime}, \tau} \frac{n_{F}\left(E_{\mathbf{k} \alpha}^{(\tau, s)}\right)-n_{F}\left(E_{\mathbf{k}+\mathbf{q} \beta}^{\left(\tau, s^{\prime}\right)}\right)}{E_{\mathbf{k} \alpha}^{(\tau, s)}-E_{\mathbf{k}+\mathbf{q} \beta}^{\left(\tau, s^{\prime}\right)}+i \eta},
$$

with matrix elements

$$
\mathscr{W}_{i j\left(\mathbf{k}, \mathbf{k}^{\prime}, \alpha, \beta\right)}^{\left(s, s^{\prime}, \tau\right)}=\left[\left(\psi_{\mathbf{k} \alpha}^{(\tau, s)}\right)^{\dagger} \hat{J}_{i} \psi_{\mathbf{k}+\mathbf{q} \beta}^{\left(\tau, s^{\prime}\right)}\right]\left[\left(\psi_{\mathbf{k}+\mathbf{q} \beta}^{\left(\tau, s^{\prime}\right)}\right)^{\dagger} \hat{J}_{j} \psi_{\mathbf{k} \alpha}^{(\tau, s)}\right]
$$


Here $n_{\mathrm{F}}(\cdot)$ denotes the Fermi function. For our cases of interest, the two valleys make identical contributions to the spin susceptibility, hence we can account for the valley degree of freedom by a degeneracy factor $g_{v}=2$. We only include contributions to the spin susceptibility that involve intra-valley excitations, as has been done in a recent calculation of the charge response (Scholz et al., 2013). In principle, inter-valley terms exist, but these are oscillating rapidly in real- space (Brey et al., 2007) and are therefore only relevant for physical observables on microscopic scales.

It follows from the structure of the spin matrices, Eqs. (274), that the in-plane components $\chi_{x x}(\mathbf{q})=\chi_{y y}(\mathbf{q})$ contain contributions only for $s \neq s^{\prime}$. In contrast, $\chi_{z z}(\mathbf{q})$ has only terms with $s=s^{\prime}$ contributing, thus $\chi_{z z}(\mathbf{q})$ is proportional to the Lindhard function $\chi_{0}(\mathbf{q})$ calculated in Ref. (Scholz et al., 2013). By similar arguments, it can be established that all off-diagonal elements of the spinsusceptibility tensor vanish. As the Hamiltonian (275) has axial symmetry, it follows that the spin susceptibility depends only on the magnitude $q \equiv|\mathbf{q}|$ of the wave vector $\mathbf{q}$.

\subsection{SPIN SUSCEPTIBILITY OF ELECTRONS: EXTRINSIC VS. INTRINSIC CON- TRIBUTIONS}

In this Section, we consider the situation where the Fermi energy is above the conduction-band edge, i.e., $\mathrm{E}_{\mathrm{F}}>\Delta / 2$. As in the previously considered case of the dielectric polarizability of monolayer graphene (Ando, 2006; Hwang and Das Sarma, 2007; Pyatkovskiy, 2009; Scholz and Schliemann, 2011; Scholz et al., 2012), the spin-response function of the electron-doped system can be separated into an extrinsic contribution that is entirely due to the occupied states in the conduction band and the intrinsic contribution arising from the completely filled valence band. In order to calculate the contribution of these transitions we start by calculating the spin susceptibility tensor for intraband and interband transitions. 

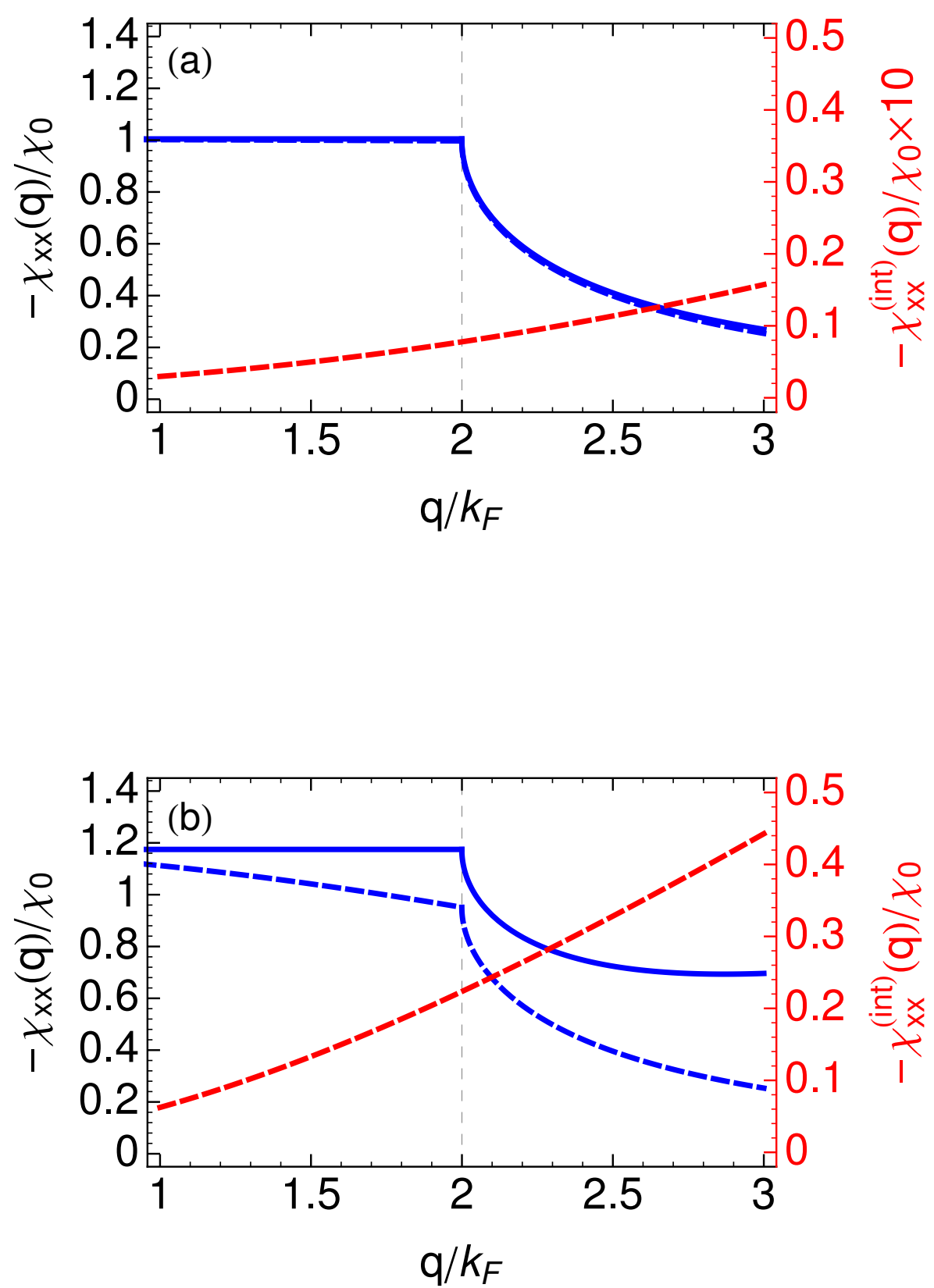

Figure 52: In-plane component $\chi_{x x}(q) \equiv \chi_{x x}^{(\text {ext })}(q)+\chi_{x x}^{(\text {int })}(q)$ of the wave-vectordependent spin-susceptibility tensor for electron-doped monolayer $\mathrm{MoS}_{2}$ (blue solid curves). We also plot the extrinsic (intrinsic) contributions $\chi_{x x}^{(e x t)}$ $\left(\chi_{x x}^{(\text {int })}\right)$ separately as the blue (red) dashed curves. Panel (a) [(b)] is for electron density $\mathrm{n}=1 \times 10^{12} \mathrm{~cm}^{-2}\left[5 \times 10^{13} \mathrm{~cm}^{-2}\right]$. Notice the sharp feature exhibited by $\chi_{x x}$ and $\chi_{x x}^{(\text {ext })}$ for $q \approx 2 k_{F}$ and the different ordinate scale for $\chi_{x x}^{(i n t)}$. 
The wave vector dependent spin susceptibility tensor for intraband transitions in electron doped system $(\alpha=\beta=+)$ is given by

$$
\chi_{i j}^{\text {intra }}(\mathbf{q})=\sum_{s, s^{\prime}, \tau} \int \frac{d^{2} k}{(2 \pi)^{2}} \mathscr{W}_{i j(\mathbf{k}, \mathbf{k}+\mathbf{q},+,+)}^{s, s^{\prime}, \tau} \frac{n_{F}\left(E_{\mathbf{k}+}^{(\tau, s)}\right)-n_{F}\left(E_{\mathbf{k}+\mathbf{q}+}^{\left(\tau, s^{\prime}\right)}\right)}{E_{\mathbf{k}+}^{(\tau, s)}-E_{\mathbf{k}+\mathbf{q}+}^{\left(\tau, s^{\prime}\right)}+i \eta},
$$

Having certain symmetries in our problem enables us to simplify the spin susceptibility tensor. First changing variable $\mathbf{k} \rightarrow \mathbf{k}-\mathbf{q}$ in term proportional to $n_{F}\left(E_{\mathbf{k}+\mathbf{q}+}^{\left(\tau, s^{\prime}\right)}\right)$ leaves us with

$$
\begin{aligned}
& \chi_{i j}^{\text {intra }}(\mathbf{q})=\sum_{s, s^{\prime}, \tau} \int \frac{d^{2} k}{(2 \pi)^{2}}\left(\mathscr{W}_{i j(\mathbf{k}, \mathbf{k}+\mathbf{q},+,+)}^{s, s^{\prime}, \tau} \frac{n_{F}\left(E_{\mathbf{k}+}^{(\tau, s)}\right)}{E_{\mathbf{k}+}^{(\tau, s)}-E_{\mathbf{k}+\mathbf{q}+}^{\left(\tau, s^{\prime}\right)}+i \eta}\right. \\
& +\left[\left(\psi_{\mathbf{k}-\mathbf{q}+}^{(\tau, s)}\right)^{\dagger} \hat{J}_{i} \psi_{\mathbf{k}+}^{\left(\tau, s^{\prime}\right)}\right]\left[\left(\psi_{\mathbf{k}+}^{\left(\tau, s^{\prime}\right)}\right)^{\dagger} \hat{J}_{j} \psi_{\mathbf{k}-\mathbf{q}+}^{(\tau, s)}\right] \\
& \left.\times \frac{n_{F}\left(E_{\mathbf{k}+}^{\left(\tau, s^{\prime}\right)}\right)}{E_{\mathbf{k}+}^{(\tau, s)}-E_{\mathbf{k}-\mathbf{q}+}^{\left(\tau, s^{\prime}\right)}+i \eta}\right),
\end{aligned}
$$

Second, due to axial symmetry in our system changing the azimuthal angle for $\mathbf{q}$ and $\mathbf{k}$ as $\theta_{\mathbf{q}}=0$ and $\theta_{k} \rightarrow \pi+\theta_{k}$ transforms $\mathbf{k}-\mathbf{q} \rightarrow \mathbf{k}+\mathbf{q}$ and some straightforward calculations yield to

$$
x_{i j}^{\text {intra }}(\mathbf{q})=\sum_{\substack{s, s^{\prime}, \tau \\ \delta= \pm 1}} \int \frac{d^{2} k}{(2 \pi)^{2}} \mathscr{W}_{i j(k, k+q,+,+)}^{s, s^{\prime}, \tau} \frac{n_{F}\left(E_{\mathbf{k}+}^{(\tau, s)}\right)}{E_{\mathbf{k}+}^{(\tau, s)}-E_{\mathbf{k}+\mathbf{q}+}^{\left(\tau, s^{\prime}\right)}+i \eta \delta},
$$

For interband transitions $(\alpha \neq \beta)$ the spin susceptibility has the following form

$$
\chi_{i j}^{\text {inter }}(\mathbf{q})=\sum_{s, s^{\prime}, \tau} \int \frac{d^{2} k}{(2 \pi)^{2}} \mathscr{W}_{i j(\mathbf{k}, \mathbf{k}+\mathbf{q},+,-)}^{s, s^{\prime}, \tau} \frac{n_{F}\left(E_{\mathbf{k}+}^{(\tau, s)}\right)-n_{F}\left(E_{\mathbf{k}+\mathbf{q}-}^{\left(\tau, s^{\prime}\right)}\right)}{E_{\mathbf{k}+}^{(\tau, s)}-E_{\mathbf{k}+\mathbf{q}-}^{\left(\tau, s^{\prime}\right)}+i \eta},
$$

In the zero-temperature limit (which we employ in the following), the Fermi functions are $n_{F}\left(E_{k+}^{(\tau, s)}\right)=\Theta\left(k_{F}^{(s \tau)}-k\right)$ and $n_{F}\left(E_{k-}^{(\tau, s)}\right)=1$, respectively. Since we considered the electron doped case, the valance band is fully occupied and thus the Fermi function $n_{\mathrm{F}}\left(\mathrm{E}_{\mathbf{k}-}^{(\tau, s)}\right)=1$ for bands with different spin indices. Using the 
same approach we used above we simplify the spin susceptibility tensor for inter band transitions into:

$$
\chi_{i j}^{\text {inter }}(\mathbf{q})=\sum_{\substack{s, s^{\prime}, \tau \\ \delta= \pm 1}} \int \frac{d^{2} k}{(2 \pi)^{2}} \mathscr{W}_{i j(\mathbf{k}, \mathbf{k}+\mathbf{k},+,-)}^{s, \tau} \frac{n_{F}\left(E_{\mathbf{k}+}^{(\tau, s)}\right)-1}{E_{\mathbf{k}+}^{(\tau, s)}-E_{\mathbf{k}+\mathbf{q}-}^{\left(\tau, s^{\prime}\right)}+i \eta \delta},
$$

Here the term with Fermi function equal to one corresponds to an intrinsic transition where the electron band is empty or equivalently the system is undoped. Therefore, we can separate the intrinsic and extrinsic part of the susceptibility. For the non-vanishing diagonal elements, we find $\chi_{j j}(\mathbf{q})=\chi_{j j}^{(\mathrm{ext})}(\mathbf{q})+\chi_{j j}^{(\mathrm{int})}(\mathbf{q})$, with

$$
\begin{aligned}
& \chi_{j j}^{(e x t)}(\mathbf{q})=\sum_{\substack{s, s^{\prime}, \tau \\
\delta= \pm 1}} \int \frac{d^{2} k}{(2 \pi)^{2}} n_{F}\left(E_{k+}^{(\tau, s)}\right)
\end{aligned}
$$

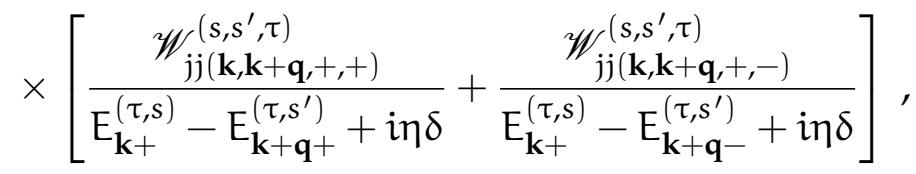

$$
\begin{aligned}
& \chi_{j j}^{(i n t)}(\mathbf{q})=-\sum_{\substack{s, s^{\prime}, \tau \\
\delta= \pm 1}} \int \frac{d^{2} k}{(2 \pi)^{2}} \frac{\mathscr{W}_{j j}^{\left(s, s^{\prime}, \tau\right)}}{E_{\mathbf{k}+\mathbf{k}+\mathbf{q},+,-)}^{(\tau, s)}-E_{k}^{\left(\tau, s^{\prime}\right)}+i \eta \mathbf{q} \delta}
\end{aligned}
$$

\subsubsection{In-plane spin-susceptibility component $\chi_{x x}$}

An explicit calculation of the extrinsic contribution to the in-plane spin susceptibility tensor element yields 


$$
\begin{aligned}
\chi_{x x}^{(\text {ext })}(q)= & -\frac{\chi_{0}}{4}\left\{\frac{2 \bar{q}^{4}+\mathcal{F} \bar{\lambda}^{2}(6+\bar{\lambda}+\mathcal{G})-\bar{q}^{2}[\mathcal{G} \mathcal{F}+\bar{\lambda}(\mathcal{F}+8 \bar{\lambda})]}{\left(\bar{q}^{2}-\bar{\lambda}^{2}\right)^{2}}\right. \\
+ & 2\left(\sqrt{4\left(\bar{k}_{\mathrm{F}}^{(+)}\right)^{2}+m_{+}^{2}}-\mathrm{m}_{+}\right)+\frac{\left[\overline{\mathrm{q}}^{6}-2 \overline{\mathrm{q}}^{2} \bar{\lambda}^{2}+2 \bar{\lambda}^{4}\left(\bar{\lambda}^{2}-4\right)-\overline{\mathrm{q}}^{4}\left(\bar{\lambda}^{2}+4\right)\right]}{\left(\bar{\lambda}^{2}-\overline{\mathrm{q}}^{2}\right)^{5 / 2}} \\
& \left.\times \log \frac{\overline{\mathrm{q}}^{2}\left(\sqrt{\bar{\lambda}^{2}-\overline{\mathrm{q}}^{2}}-2\right)}{\mathcal{F} \sqrt{\bar{\lambda}^{2}-\overline{\mathrm{q}}^{2}}+\bar{\lambda}^{2}\left(\mathcal{G}-\mathrm{m}_{+}\right)-\overline{\mathrm{q}}^{2}(\mathcal{G}+\bar{\lambda})}\right\}
\end{aligned}
$$

with $\chi_{0}=g_{v} g_{s} k_{0}^{2} /\left(2 \pi E_{0}\right) \equiv \Delta /\left(\pi a^{2} t^{2}\right)$. We have also used the abbreviations

$$
\begin{gathered}
\mathcal{F}=\sqrt{\bar{q}^{4}-2 \bar{q}^{2} \bar{\lambda}\left(\mathcal{G}-m_{+}\right)-2 \bar{\lambda}^{2} m_{+}\left(\mathcal{G}-m_{+}\right)+4 \kappa_{\mathrm{q}}^{2}\left(\bar{\lambda}^{2}-\bar{q}^{2}\right)}, \\
\mathcal{G}=\sqrt{4 \kappa_{\mathrm{q}}^{2}+\mathrm{m}_{+}^{2}}, \\
\kappa_{\mathrm{q}}=\mathcal{K}_{\mathrm{q}} \Theta\left(k_{\mathrm{F}}^{(+)}+\mathrm{k}_{\mathrm{F}}^{(-)}-\mathrm{q}\right)+\bar{k}_{\mathrm{F}}^{(+)} \Theta\left(\mathrm{q}-\mathrm{k}_{\mathrm{F}}^{(+)}-\mathrm{k}_{\mathrm{F}}^{(-)}\right), \\
\mathcal{K}_{\mathrm{q}}=\frac{\overline{\mathrm{q}}^{3}-\overline{\mathrm{q}} \bar{\lambda}(\bar{\lambda}-2)-\overline{\mathrm{q}} \bar{\lambda} \sqrt{4+\bar{q}^{2}-\bar{\lambda}^{2}}}{2\left(\overline{\mathrm{q}}^{2}-\bar{\lambda}^{2}\right)} .
\end{gathered}
$$

In the limit $\mathrm{q} \rightarrow 0$, the result

$$
\chi_{x x}^{(\text {ext })}(0)=-\frac{\chi_{0}}{2}\left(\frac{\left(4-\bar{\lambda}^{2}\right) \operatorname{arctanh} \frac{\bar{\lambda}}{2}}{\bar{\lambda}}+\sqrt{4\left(\bar{k}_{\mathrm{F}}^{(+)}\right)^{2}+m_{+}^{2}}-m_{+}\right)
$$

is found.

The intrinsic contribution can be expressed as

$$
\chi_{x x}^{(\text {int })}(q)=-\left.\chi_{x x}^{(\mathrm{ext})}(q)\right|_{\kappa_{q} \equiv \mathcal{K}_{q}}+\chi_{x x}^{(\mathrm{ext})}(0)+\chi_{x x}^{(\mathrm{int})}(0)
$$

with $\chi_{x x}^{(\mathrm{ext})}(0)$ from Eq. (300). In contrast to the static dielectric polarizability of both monolayer $\mathrm{MoS}_{2}$ (Scholz et al., 2013) and monolayer graphene (Ando, 2006; Hwang and Das Sarma, 2007; Pyatkovskiy, 2009; Scholz and Schliemann, 2011; 

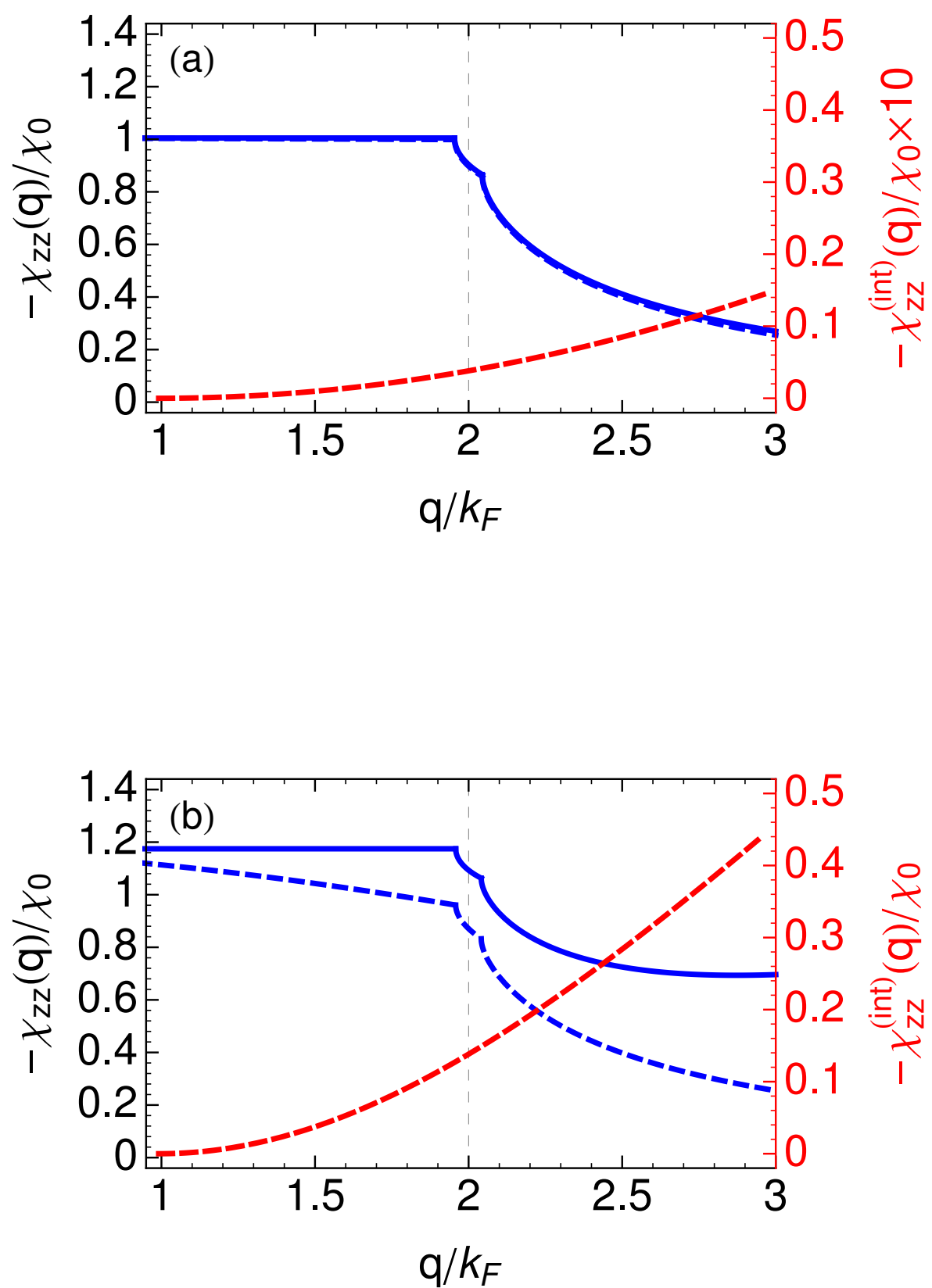

Figure 53: Spin-susceptibility component $\chi_{z z}(q) \equiv \chi_{z z}^{(\text {ext })}(q)+\chi_{z z}^{(\text {int })}(q)$ for electrondoped monolayer $\mathrm{MoS}_{2}$ (blue solid curves). The extrinsic (intrinsic) contributions $\chi_{z z}^{(\mathrm{ext})}\left(\chi_{z z}^{(\mathrm{int})}\right)$ are also plotted as the blue (red) dashed curves. Panel (a) [(b)] shows results for electron density $\mathrm{n}=1 \times 10^{12} \mathrm{~cm}^{-2}\left[5 \times 10^{13} \mathrm{~cm}^{-2}\right]$. Sharp features are exhibited by $\chi_{z z}$ and $\chi_{z z}^{(\text {ext })}$ for $q=k_{\mathrm{F}}^{(+)}, k_{\mathrm{F}}^{(-)}$. Note the different scale for $\chi_{z z}^{(\mathrm{int})}$. 
Scholz et al., 2012), the in-plane spin susceptibility of electron-doped transition metal dichalcogenides is found to have a finite intrinsic contribution for $q \rightarrow 0$,

$$
\chi_{x x}^{(\text {int) }}(0)=-\frac{\chi_{0}}{2}\left(\frac{2 \bar{\lambda}-\left(4-\bar{\lambda}^{2}\right) \operatorname{arctanh} \frac{\bar{\lambda}}{2}}{\bar{\lambda}}\right)
$$

As the expression (302) vanishes for $\lambda \rightarrow 0$, the finite $\chi_{x x}^{(\mathrm{int})}(0)$ is a SOC effect. Combining Eqs. (302) and (300) yields the $q \rightarrow 0$ limit of the in-plane spinsusceptibility tensor component in the electron-doped case given by

$$
\begin{aligned}
\chi_{x x}(0) & =-\frac{\chi_{0}}{2}\left(\sqrt{4\left(\bar{k}_{\mathrm{F}}^{(+)}\right)^{2}+m_{+}^{2}}+\bar{\lambda}\right), \\
& \equiv-\chi_{0} \frac{E_{F}}{E_{0}} .
\end{aligned}
$$

Figure 52 illustrates the behavior of $\chi_{x x}(q)$ and also shows the individual extrinsic and intrinsic contributions. A cancellation of the latters' $q$ dependences yields a constant $\chi_{x x}(q)$ for $q \leqslant k_{F}^{(+)}+k_{F}^{(-)}\left(\approx 2 k_{F}\right.$ typically), which is followed by an abrupt decrease of the spin susceptibility for $q>k_{F}^{(+)}+k_{F}^{(-)}$. The general line shape is similar to the one found for an ordinary $2 \mathrm{D}$ electron gas (Giuliani and Vignale, 2005) in the absence of spin-orbit coupling, and the plateau behavior is also exhibited by response functions of monolayer graphene (Ando, 2006; Hwang and Das Sarma, 2007; Pyatkovskiy, 2009; Scholz and Schliemann, 2011). The fact that only a single sharp feature appears in $\chi_{x x}(q)$, even though there are two Fermi surfaces for the values of density used for the plots, originates from the in-plane response being governed by transitions between eigenstates with opposite $s_{z}$ quantum number. Furthermore, in contrast to the case of an ordinary 2D electron gas, the plateau value of $\chi_{x x}(q)$ is density dependent [see Eq. (303)], but this dependence is much weaker than in the case of graphene (Ando, 2006; Hwang and Das Sarma, 2007; Pyatkovskiy, 2009; Scholz and Schliemann, 2011) because of the relatively large value of the gap parameter $\Delta$. 


\subsubsection{Perpendicular spin-susceptibility component $\chi_{z z}$}

The extrinsic contribution to the spin-susceptibility component describing the response in the direction perpendicular to the $2 \mathrm{D}$ material's plane is the sum of terms arising from the individual spin-split bands,

$$
\begin{aligned}
& \chi_{z z}^{(\mathrm{ext})}(\mathrm{q})=\sum_{s} \chi_{z z, s}^{(\mathrm{ext})}(\mathrm{q}) \\
& \chi_{z z, s}^{(e x t)}(q)=\frac{\chi_{0}}{8}\left\{m_{s}-2 \sqrt{4\left(\bar{k}_{F}^{(s)}\right)^{2}+m_{s}^{2}}-\frac{\left(m_{s}^{2}-\bar{q}^{2}\right)}{\bar{q}} \arctan \frac{\bar{q}}{m_{s}}\right\} \Theta\left(2 k_{F}^{(s)}-q\right) \\
& +\frac{\chi_{0}}{8}\left\{m_{s}-2 \sqrt{\left(\bar{k}_{\mathrm{F}}^{(s)}\right)^{2}+m_{s}^{2}}+\frac{1}{\bar{q}} \sqrt{\left[4\left(\bar{k}_{\mathrm{F}}^{(s)}\right)^{2}+m_{s}^{2}\right]\left[\bar{q}^{2}-4\left(\bar{k}_{\mathrm{F}}^{(s)}\right)^{2}\right]}\right. \\
& -\frac{m_{s}^{2}-\bar{q}^{2}}{\bar{q}}\left[2 \arcsin \sqrt{\frac{4\left(\bar{k}_{F}^{(s)}\right)^{2}+m_{s}^{2}}{m_{s}^{2}+\bar{q}^{2}}}-2 \arctan \frac{m_{s}}{\bar{q}}+\frac{1}{2} \arctan \left(\frac{m_{s}}{2 \bar{q}}-\frac{\bar{q}}{2 m_{s}}\right)\right. \\
& \left.\left.-\frac{1}{2} \arctan \frac{8\left(\bar{k}_{\mathrm{F}}^{(s)}\right)^{2}+m_{s}^{2}-\bar{q}^{2}}{2 \sqrt{4\left(\bar{k}_{\mathrm{F}}^{(s)}\right)^{2}+m_{s}^{2}} \sqrt{\bar{q}^{2}-4\left(\bar{k}_{\mathrm{F}}^{(s)}\right)^{2}}}\right]\right\}\left(q-2 k_{\mathrm{F}}^{(s)}\right) \text {. }
\end{aligned}
$$

In the $q \rightarrow 0$ limit, Eq. (304a) yields

$$
\chi_{z z}^{(\mathrm{ext})}(0)=-\frac{\chi_{0}}{4} \sum_{\mathrm{s}} \sqrt{4\left(\bar{k}_{\mathrm{F}}^{(\mathrm{s})}\right)^{2}+\mathrm{m}_{\mathrm{s}}^{2}},
$$

thus $-\chi_{z z}^{(\mathrm{ext})}(0)$ corresponds to the density of states at the Fermi energy. For the intrinsic contribution, the expression

$$
\chi_{z z}^{(\text {int })}(q)=-\frac{\chi_{0}}{8} \sum_{s}\left[m_{s}-\frac{m_{s}^{2}-\bar{q}^{2}}{\bar{q}} \arctan \frac{\bar{q}}{m_{s}}\right]
$$

is found, which vanishes in the limit $q \rightarrow 0$. As a result, $\chi_{z z}(0) \equiv \chi_{z z}^{(\mathrm{ext})}(0)$, and we find using Eq. (305)

$$
\chi_{z z}(0)=-\chi_{0} \frac{E_{F}}{E_{0}} \equiv \chi_{x x}(0)
$$

The line shape of $\chi_{z z}(q)$ is shown in Fig. 53 for band-structure parameters of $\mathrm{MoS}_{2}$ and two density values. As in the case of the in-plane spin-susceptibility 
component, a cancellation of $\mathrm{q}$ dependences from the extrinsic and intrinsic contributions results in a plateau for $\chi_{z z}(q)$ for wave vectors smaller than a threshold value (here: $2 k_{F}^{(+)}$). While the plateau value is the same as for $\chi_{x x}(q)$, its width is different. Also in contrast to the behavior of $\chi_{x x}(q)$, two sharp features at $q=k_{F}^{(+)}$ and at $\mathrm{q}=\mathrm{k}_{\mathrm{F}}^{(-)}$signify the existence of the two Fermi surfaces. However, the line shapes of the in-plane and perpendicular spin-susceptibility components become very similar again for $\mathrm{q}>2 \mathrm{k}_{\mathrm{F}}^{(-)}$. Hence, except for wave vectors within the region close to the two Fermi wave vectors, the spin response of charge carriers in electron-doped transition metal dichalcogenides is isotropic and very similar to that of an ordinary 2D electron gas (Giuliani and Vignale, 2005). Differences to the standard behavior will therefore occur in the oscillations of the spin susceptibility in real space [Eq. (287)] whose wave length and beating pattern is governed by the sharp features in $\chi_{i j}(q)$.

\subsection{SPIN SUSCEPTIBILITY OF HOLES: IN-PLANE/ OUT-OF-PLANE ANISOTROPY}

Specializing the general definition (288a) for the spin-susceptibility tensor to the situation where only states in the valence band are occupied [i.e., for $n_{\mathrm{F}}\left(\mathrm{E}_{\mathbf{k}+}^{(\tau, s)}\right) \equiv$ 0], we obtain

$$
\chi_{j j}(\mathbf{q})=\sum_{\substack{s, s^{\prime}, \tau \\ \delta= \pm 1}} \int \frac{d^{2} k}{(2 \pi)^{2}} n_{F}\left(E_{\mathbf{k}-}^{(\tau, s)}\right)\left[\frac{\mathscr{W}_{j j\left(s^{\prime}, \mathbf{k}+\mathbf{q},-,-\right)}^{\left(s, s^{\prime},\right)^{\prime}}}{E_{\mathbf{k}-}^{(\tau, s)}-E_{\mathbf{k}+\mathbf{q}-}^{\left(\tau, s^{\prime}\right)}+i \eta \delta}+\frac{\mathscr{W}_{j j(\mathbf{k}, \mathbf{k}+\mathbf{q},-,+)}^{\left(s, s^{\prime}, \tau\right)}}{E_{\mathbf{k}-}^{(\tau, s)}-E_{\mathbf{k}+\mathbf{q}+}^{\left(\tau, s^{\prime}\right)}+i \eta \delta}\right] .
$$

Note the analogy of the expression (308) with that of the extrinsic part of the electron-doped case [cf. Eq. (294)]. If we were to adopt the hole picture by defining $\tilde{n}_{\mathrm{F}}=1-\mathfrak{n}_{\mathrm{F}}$ as the distribution function of charge carriers, the expression (308) could be written, in full analogy to the electron-doped case, as the sum of the intrinsic contribution and an extrinsic part that vanishes in the limit of zero hole density. While we have used the electron picture throughout, the formulae given in our work make it possible to easily find the equivalent results for the hole picture. 

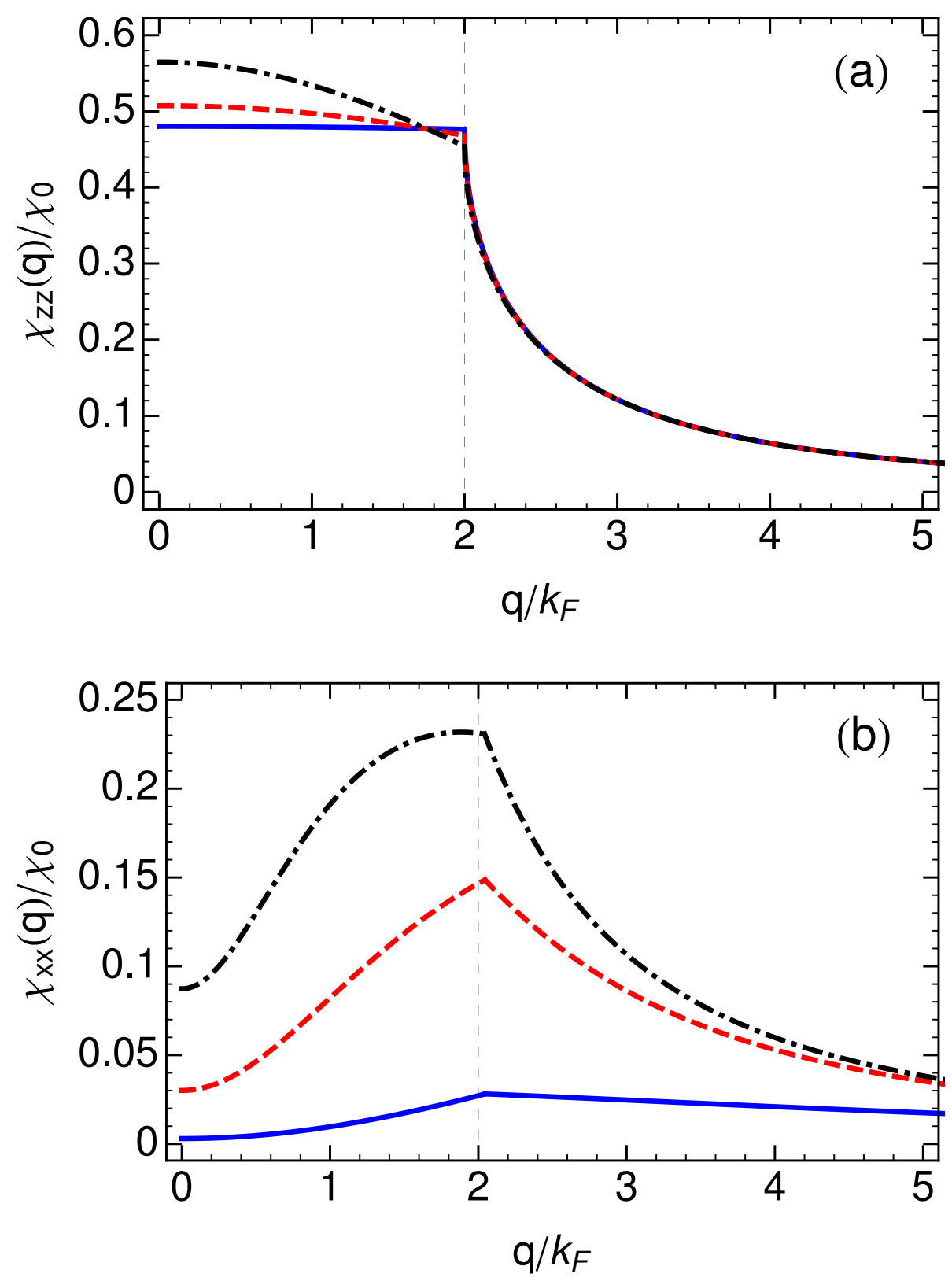

Figure 54: Spin response of hole-doped monolayer $\mathrm{MoS}_{2}$. Panel (a) [(b)] shows $\chi_{z z}(q)$ $\left[\chi_{x x}(q)\right]$ obtained for a hole sheet density $\mathrm{n}=1 \times 10^{12} \mathrm{~cm}^{-2}$ (blue solid curve), $\mathrm{n}=1 \times 10^{13} \mathrm{~cm}^{-2}$ (red dashed curve), and $\mathrm{n}=3 \times 10^{13} \mathrm{~cm}^{-2}$ (black dot-dashed curve). Noteworthy features are the strong in-plane/out-of-plane anisotropy of the spin response, the deviations from ideal 2D-electron-gas behavior even for $\chi_{z z}(q)$, which are getting more pronounced as the density increases, and the nonanalyticity exhibited by $\chi_{z z}(q)\left[\chi_{x x}(q)\right]$ for $q=2 k_{F} \equiv$ $2 k_{\mathrm{F}}^{(+)}\left[\mathrm{q}=\mathrm{k}_{1}>2 \mathrm{k}_{\mathrm{F}}^{(+)}\right]$. 
In the following, we consider the situation where hole densities $\mathrm{n}$ are small enough such that only the upper-most of the two spin-split valence bands has empty states. This implies $\Delta / 2-\lambda<-\mathrm{E}_{\mathrm{F}}<\Delta / 2+\lambda$, and there will be only one Fermi surface with radius $k_{\mathrm{F}}^{(+)} \equiv \sqrt{4 \pi \mathrm{n} / \mathrm{g}_{v}}$. For this situation, we obtain in the zero-temperature limit the in-plane component of the spin-susceptibility tensor as

$$
\begin{aligned}
& \chi_{x x}(q)= \\
& \frac{\chi_{0}}{8}\left\{\frac{2 \overline{\mathrm{q}}^{4}(1-\bar{\lambda})+\bar{\lambda}^{2}\left[\mathcal{H}(\tilde{\mathcal{G}}-6-\bar{\lambda})+4 \bar{\lambda}\left(4-\bar{\lambda}^{2}\right)\right]+\overline{\mathrm{q}}^{2}\left[\bar{\lambda}\left(8+\mathcal{H}-8 \bar{\lambda}+6 \bar{\lambda}^{2}\right)-\tilde{\mathcal{G}} \mathcal{H}\right]}{\left(\overline{\mathrm{q}}^{2}-\bar{\lambda}^{2}\right)^{2}}\right. \\
& +2\left(\sqrt{4\left(\bar{k}_{\mathrm{F}}^{(+)}\right)^{2}+\mathrm{m}_{+}^{2}}-\mathrm{m}_{+}\right)+\frac{\left[\overline{\mathrm{q}}^{6}-2 \overline{\mathrm{q}}^{2} \bar{\lambda}^{2}+2 \bar{\lambda}^{4}\left(\bar{\lambda}^{2}-4\right)-\overline{\mathrm{q}}^{4}\left(\bar{\lambda}^{2}+4\right)\right]}{\left(\bar{\lambda}^{2}-\overline{\mathrm{q}}^{2}\right)^{5 / 2}} \\
& \left.\times \log \frac{\overline{\mathrm{q}}^{2}\left(2 \bar{\lambda}-2+\sqrt{\bar{\lambda}^{2}-\overline{\mathrm{q}}^{2}}\right)+2 \bar{\lambda} \mathrm{m}_{+}\left(\sqrt{\overline{\bar{\lambda}}^{2}-\overline{\mathrm{q}}^{2}}+\bar{\lambda}\right)}{\mathcal{H} \sqrt{\bar{\lambda}^{2}-\overline{\mathrm{q}}^{2}}+\bar{\lambda}^{2}\left(\mathrm{~m}_{+}+\tilde{\mathcal{G}}\right)+\overline{\mathrm{q}}^{2}(\bar{\lambda}-\tilde{\mathcal{G}})}\right\} .
\end{aligned}
$$

We have again introduced abbreviations

$$
\begin{aligned}
\mathcal{H}= & \sqrt{\overline{\mathrm{q}}^{4}+2 \overline{\mathrm{q}}^{2} \bar{\lambda}\left(\tilde{\mathcal{G}}^{2} \mathrm{~m}_{+}\right)+2 \bar{\lambda}^{2} \mathrm{~m}_{+}\left(\tilde{\mathfrak{G}}^{2} \mathrm{~m}_{+}\right)+4 \tilde{\mathrm{k}}_{\mathrm{q}}^{2}\left(\bar{\lambda}^{2}-\overline{\mathrm{q}}^{2}\right)}, \\
\tilde{\mathcal{G}} & =\sqrt{4 \tilde{\mathrm{k}}_{\mathrm{q}}^{2}+\mathrm{m}_{+}^{2}}, \\
\tilde{\mathrm{k}}_{\mathrm{q}} & =\mathcal{K}_{\mathrm{q}} \Theta\left(\mathrm{k}_{1}-\mathrm{q}\right)+\overline{\mathrm{k}}_{\mathrm{F}}^{(+)} \Theta\left(\mathrm{q}-\mathrm{k}_{1}\right), \\
\frac{\mathrm{k}_{1}}{\mathrm{k}_{0}} & =\overline{\mathrm{k}}_{\mathrm{F}}^{(+)}+\left[\left(\overline{\mathrm{k}}_{\mathrm{F}}^{(+)}\right)^{2}+\bar{\lambda}\left(\sqrt{4\left(\overline{\mathrm{k}}_{\mathrm{F}}^{(+)}\right)^{2}+\mathrm{m}_{+}^{2}}-\mathrm{m}_{+}\right)\right]^{\frac{1}{2}},
\end{aligned}
$$

with $\mathcal{K}_{\mathrm{q}}$ from Eq. (299). Note that $\chi_{x x}(q)$ is nonanalytic at $q=k_{1}\left(>2 k_{F}^{(+)}\right)$. In the limit $q \rightarrow 0$, Eq. (309) yields

$$
\chi_{x x}(0)=\frac{\chi_{0}}{4}\left(\sqrt{4\left(\bar{k}_{\mathrm{F}}^{(+)}\right)^{2}+m_{+}^{2}}-m_{+}\right) .
$$

The general result for the spin-susceptibility tensor component perpendicular to the plane is obtained as

$$
\chi_{z z}(q)=-\sum_{s} \chi_{z z, s}^{(e x t)}(q) \Theta\left(k_{F}^{(s)}\right)
$$


with the expression for $\chi_{z z, s}^{(\text {ext) }}$ given in Eq. (304b). Unlike in the electron-doped case, SOC does not give rise to the existence of two Fermi surfaces for all hole densities. For our case of interest where hole densities are low enough such that only the upper-most valence band has empty states, only a single Fermi surface exists. In this situation, the $\mathrm{q} \rightarrow 0$ limit yields

$$
\chi_{z z}(0)=\frac{\chi_{0}}{4} \sqrt{4\left(\bar{k}_{F}^{(+)}\right)^{2}+m_{+}^{2}} \equiv \frac{\chi_{0}}{2}\left(-\frac{E_{F}}{E_{0}}+\frac{\bar{\lambda}}{2}\right)
$$

which corresponds to the density of states in the upper-most valence band. In contrast to the in-plane spin-susceptibility component, $\chi_{z z}(q)$ is non-analytic at $\mathrm{q}=2 \mathrm{k}_{\mathrm{F}}^{(+)}$. Also, $\chi_{z z}(0) \gg \chi_{x x}(0)$ for typical hole densities, signifying a strong anisotropy of the spin response.

The behavior of the spin response in the hole-doped case differs markedly from the electron-doped situation. See Fig. 54 for an illustration. As a first observation, a strong dependence on hole-sheet density is apparent. In the low-density regime, the in-plane spin response is almost uniformly very small, whereas $\chi_{z z}(q)$ has the line shape associated with the response functions of an ordinary $2 \mathrm{D}$ electron system (Giuliani and Vignale, 2005). As the hole density increases, a pronounced peak develops in $\chi_{x x}(q)$ for $q=k_{1} \gtrsim 2 k_{F}^{(+)}$, and the plateau behavior of $\chi_{z z}(q)$ disappears. Some of these features are very similar to those exhibited by the spin response of $2 \mathrm{D}$ hole systems (Kernreiter et al., 2013) realized by a quantumwell confinement in typical semiconductor heterostructures (Winkler, 2003). The nonanalyticity at (near) $q=2 k_{F}$ in $\chi_{z z}\left(\chi_{x x}\right)$ as well as the power-law behavior in its vicinity (She and Bishop, 2013) determine the decay of the corresponding spin-susceptibility oscillations in real space. This and other consequences of the unusual spin-response properties in the hole-doped case will be discussed in greater detail in the following Section. 


\subsection{PHYSICAL CONSEQUENCES OF UNUSUAL SPIN RESPONSE IN THE HOLE- DOPED CASE}

Based on the results presented in the previous Section, we consider spin-related physical quantities for hole-doped monolayers of transition metal dichalcogenides. We start by discussing the properties of hole-carrier-mediated exchange interaction between localized impurity spins. Then the paramagnetic response of our system of interest is investigated. These examples serve to illustrate the very different behavior of hole-doped systems, in contrast to the electron-doped case that mirrors the properties of ordinary $2 \mathrm{D}$ electron gases.

\subsubsection{RKKY interaction and mean-field magetism}

We consider two localized impurity spins $\mathbf{I}^{(1)}$ and $\mathbf{I}^{(2)}$ that couple via a contact interaction of strength $J$ to the local spin density of holes in a monolayer transition metal dichalcogenide sample. In second-order perturbation theory, such a coupling gives rise to an effective exchange interaction between the impurity-spin components that is described by the RKKY Hamiltonian (Ruderman and Kittel, 1954; Kasuya, 1956; Yosida, 1957)

$$
\mathrm{H}_{\mathrm{RKKY}}^{(1,2)}=-\mathrm{J}^{2} \sum_{i, j} \mathrm{I}_{i}^{(1)} \mathrm{I}_{j}^{(2)} \chi_{i j}(\mathbf{R}) .
$$

Here $\mathbf{R}$ is the distance vector between the locations of the two impurity spins, and $\chi_{i j}(\mathbf{R})$ denotes the spin susceptibility in real space given by Eq. (287). For our cases of interest, the spin susceptibility turns out to be isotropic in its dependence on real-space position; $\chi_{i j}(\mathbf{R}) \equiv \chi_{i j}(R)$.

Figure 55 shows plots of the quantity $\left(k_{F} R\right)^{2} \chi_{j j}(R)$ for two realistic values of the hole density. The fact that $\chi_{z z}(R) \propto R^{-2}$ is clearly indicated by the constancy of the oscillations exhibited by the blue solid curves. In contrast, the in-plane response function is seen to decay faster with distance R. Closer inspection reveals that $X_{z z}(R) \propto R^{-5 / 2}$, i.e., shows behavior that deviates from the expected 

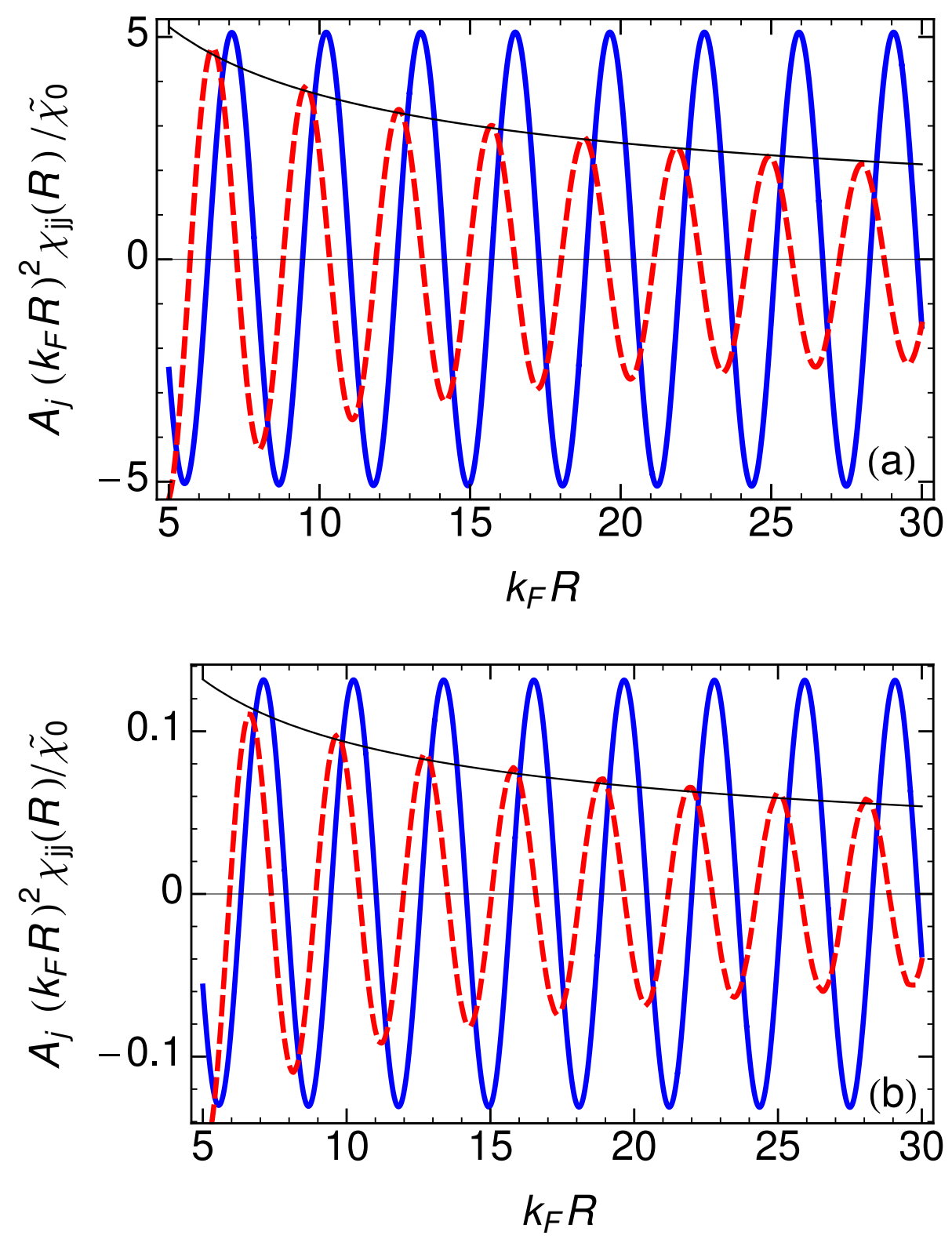

Figure 55: Spin response functions in real space. To highlight deviations from the ordinary 2D-electron-gas behavior (Giuliani and Vignale, 2005), we plot the quantities $A_{j}\left(k_{F} R\right)^{2} \chi_{j j}(R)$ in panel (a) [(b)] obtained for a hole density $n=$ $1 \times 10^{12} \mathrm{~cm}^{-2}\left[\mathrm{n}=3 \times 10^{13} \mathrm{~cm}^{-2}\right]$. The red dashed (blue solid) curve shows $\chi_{x x}\left(\chi_{z z}\right)$. The decrease of the oscillation amplitude for $\left(k_{F} R\right)^{2} \chi_{x x}(R)$ shows that the in-plane spin response decays faster than the $R^{-2}$ power law expected for ordinary $2 \mathrm{D}$ electron systems. The scaling factors are $A_{x}=4 \times 10^{4}[4]$ and $A_{z}=10^{3}[1]$ in Panel (a) [(b)], and $\tilde{\chi}_{0}=\chi_{0} k_{\mathrm{F}}^{2} /(2 \pi) \equiv 2 n k_{0}^{2} /\left(\pi \mathrm{E}_{0}\right)$. 
$\mathrm{R}^{-2}$ power law of a $2 \mathrm{D}$ Fermi liquid. Previously, deviations from the $\mathrm{R}^{-\mathrm{d}}$ powerlaw decay of the RKKY range function in a d-dimensional electron system have been shown to emerge as the result of strong electron-electron correlations (She and Bishop, 2013) or for particular placements of magnetic impurity atoms with respect to a material's crystal lattice (Kirwan et al., 2008; Uchoa et al., 2011). In contrast, the shorter-than-normal range of the in-plane spin response for $\mathrm{MoS}_{2}$ found here is exhibited by a uniform and non-interacting electron system. Thus the in-plane RKKY range function for monolayer transition metal dichalcogenides shows behavior that is intermediate between that of an ordinary $2 \mathrm{D}$ electron gas (Giuliani and Vignale, 2005) (or doped graphene (Brey et al., 2007)) and undoped graphene (Wunsch et al., 2006).

In the low-density regime, the amplitude of $\chi_{z z}(R)$ can be more than an order of magnitude larger than that of $\chi_{x x}(R)$; see Fig. 55(a). However, as the density is increased, $\chi_{x x}(R)$ becomes appreciable and even reaches the same magnitude as $\chi_{z z}(R)$; see Fig. $55($ b). From the figure, it is also apparent that the oscillations of $\chi_{x x}(R)$ and $\chi_{z z}(R)$ have a relative phase shift that varies somewhat with $R$ and sometimes turns out to be close to $\pi / 2$. It follows from this observation that the lowest-energy state of two RKKY-coupled impurity spins can change from the typically expected easy-axis configuration (both impurity spins align in the direction perpendicular to the monolayer plane) to an easy-plane alignment if the distance $R$ corresponds to a point where $\chi_{x x}(R)\left[\chi_{z z}(R)\right]$ has a maximum [a zero].

Considering now a large number of impurity spins distributed, on average, homogeneously with density $n_{I}$ in the material, the RKKY spin Hamiltonian can be treated using standard mean-field theory (Yosida, 1996). For the hole-doped situation, we have $\left.\left.\chi_{z z}(q)\right|_{q=0} \gg \chi_{x x}(q)\right|_{q=0}$, hence the spin system will exhibit Ising-type ferromagnetism with Curie temperature given by

$$
\mathrm{T}_{\mathrm{C}}=\left.\mathrm{T}_{0} \frac{\chi_{z z}(\mathrm{q})}{\chi_{0}}\right|_{\mathrm{q}=0},
$$

with the temperature scale

$$
\mathrm{T}_{0}=\frac{\mathrm{I}(\mathrm{I}+1)}{3} \frac{\mathrm{J}^{2}}{\mathrm{k}_{\mathrm{B}}} \mathrm{n}_{\mathrm{I}} \frac{\Delta}{\pi \mathrm{a}^{2} \mathrm{t}^{2}} .
$$




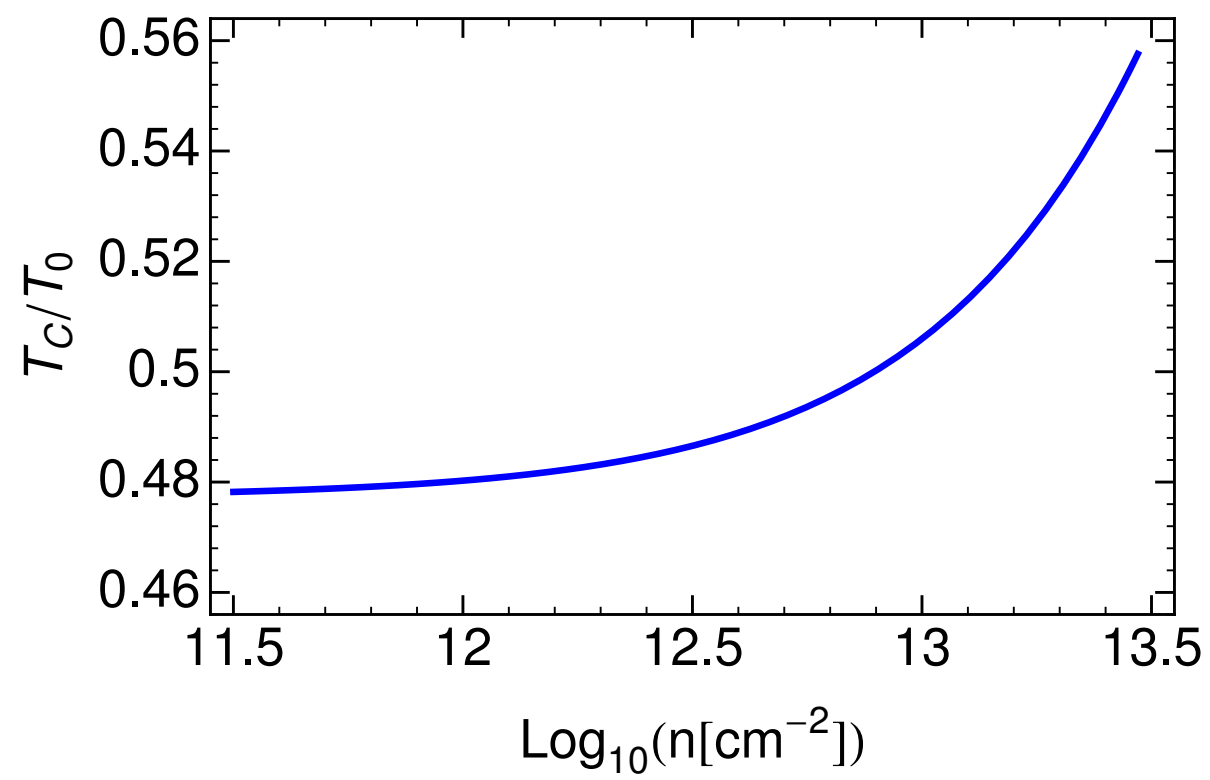

Figure 56: Density dependence of the Curie temperature for hole-mediated easy-axis Ising ferromagnetism of impurity spins in monolayer $\mathrm{MoS}_{2}$.

Here I and $n_{I}$ denote the impurity spin quantum number and areal density of impurities, respectively. Due to the density dependence of $\chi_{z z}(q=0)$ [see Eq. (316)], the Curie temperature can, in principle, be manipulated by the magnitude of hole doping. However, as illustrated in Fig. 56, the range of realistic values for the hole density allows for an adjustment of $\mathrm{T}_{\mathrm{C}}$ by only upto $10 \%$ in the highdensity regime.

In general, mean-field predictions for transition temperatures are only a crude approximation to reality, as the excitation of spin waves generally suppresses in some cases, even destroys - magnetic order. For our case of interest, the line shape of $\chi_{z z}(q)$ near $q=0$ implies (Simon et al., 2008) that spin-wave excitations cost a finite amount of energy, hence the Curie temperature should stay finite even when fluctuations are taken into account. 


\subsubsection{Pauli paramagnetism and effective g-factor}

An external magnetic field generally couples to the hole carriers' spin via a Zeeman term $\mathrm{H}_{Z}=\kappa \mu_{B} B_{j} \hat{J}_{j}$, where $\mu_{B}$ is the Bohr magneton and $2 \kappa$ the bulk valence-band $\mathrm{g}$ factor. ${ }^{1}$ In the limit of a small magnetic field, the paramagnetic susceptibility is given by

$$
\chi_{P, j}=\left.\left(\kappa \mu_{B}\right)^{2} \chi_{j j}(q)\right|_{q=0}
$$

where $\chi_{j j}(q)$ are the spin susceptibilities of the hole-doped system for the inplane and out-of-plane response whose $q \rightarrow 0$ limits are shown in Eqs. (314) and (316). It is possible to define a collective $g$-factor for the charge carriers by expressing the paramagnetic susceptibility in terms of the density of states, which is the zero-q limit of the Lindhard function (Giuliani and Vignale, 2005) $\chi_{L}(q)$, as $\chi_{P, j}=\left(g_{j} \mu_{B}\right)^{2} \chi_{L}(0) / 4$, and equate this with the expression in Eq. (319) to yield (Kernreiter et al., 2013)

$$
g_{j}=2 \kappa \sqrt{\left.\frac{\chi_{j j}(q)}{\chi_{L}(q)}\right|_{q=0}} .
$$

Our result for the out-of-plane spin response of holes in monolayer transition metal dichalcogenides implies $g_{z} \equiv 2 \kappa$, as $\chi_{z z}(q=0)$ turns out to be equal to the density of states at the Fermi energy. However, the in-plane g-factor shows unusual behavior, which is illustrated in Fig. 57. For small densities (small $k_{F}$ ), $g_{x}$ is negligible [see Eq. (314)]. In contrast, for large hole densities, $g_{x}$ can become of the same order of magnitude as $g_{z}$.

\subsection{SUMMARY AND CONCLUSIONS}

We have obtained analytical expressions for the static wave vector-dependent spin susceptibility in monolayer $\mathrm{MoS}_{2}$ for both electron and hole doped cases. We have adopted the low energy effective Hamiltonian without considering the

I We adopt a notation that is commonly $u$ used in semiconductor physics. See, e.g., Ref. (Suzuki and Hensel, 1974). 


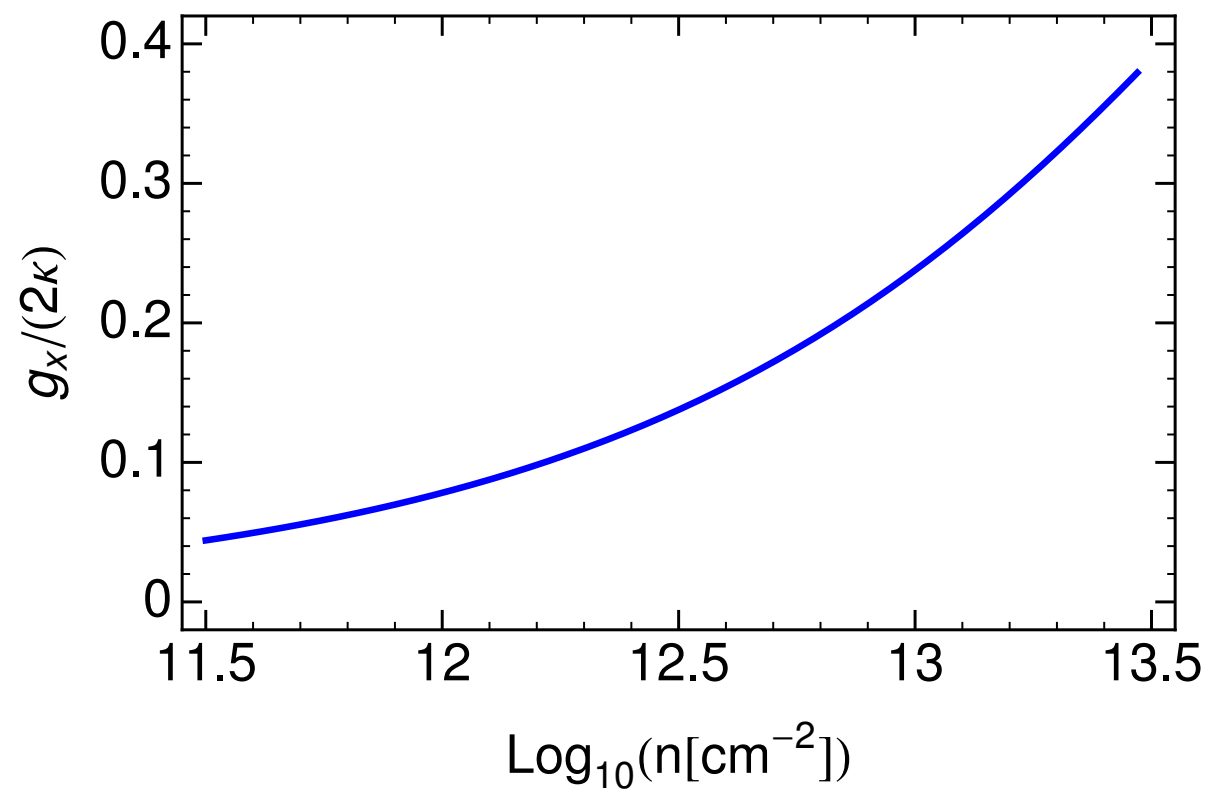

Figure 57: Density dependence of the effective in-plane g-factor for hole-doped $\mathrm{MoS}_{2}$.

correction terms introduced in previous works (Rostami et al., 2013; Kormányos et al., 2013). Within linear response theory, we found intrinsic (unroped or ungated) and extrinsic (doped or gated) contributions of the wave vector spin susceptibility components. We showed that, for the electron doped case, the in-plane spin susceptibility component $\left(\chi_{x x}(q)\right.$ and $\left.\chi_{y y}(q)\right)$ has a finite intrinsic contribution for $\mathrm{q} \rightarrow 0$. We found that this finite intrinsic contribution is a spin-orbit effect. Having a finite intrinsic contribution to the in-plane spin susceptibility is in contrast to the static dielectric polarizability of monolayer $\mathrm{MoS}_{2}$ and graphene. The in-plane spin susceptibility in electron doped case found to be constant for wave vectors $q<2 k_{F}$ due to cancelation of intrinsic and extrinsic contributions. This feature is similar to the one found in the ordinary $2 \mathrm{D}$ electron gas. However, this constant plateau, like in the case of graphene, is density dependent which is in contrast to an ordinary $2 \mathrm{D}$ electron gas. For wave vectors $q>2 k_{F}$, the constant in-plane spin susceptibility is followed by an abrupt drop. In the electron doped case, in the limit of a zero wave vector, the intrinsic contribution of the perpendicular spin susceptibility $\left(\chi_{z z}^{\text {int }}(0)\right)$ vanishes and $\chi_{z z}(0) \equiv \chi_{z z}^{\text {ext }}(0)$ . The spin susceptibility $\chi_{z z}$ shows a plateau for $q<k_{\mathrm{F}}^{+}$which is followed by 
two sharp features at $\mathrm{q}=\mathrm{k}_{\mathrm{F}}^{+}$and at $\mathrm{q}=\mathrm{k}_{\mathrm{F}}^{-}$signifying the existence of the two Fermi surfaces. For $q>k_{F}^{-}$, we see the same drop as in $\chi_{x x}$. For the hole doped case we found remarkably different behaviours from an ordinary $2 \mathrm{D}$ electron gas. First we found that the out-of-plane and in-plane response are hole density dependent. The in-plane response $\chi_{x x}$ for low hole densities is uniformly small, however as the hole density increases it shows pronounced non-analytical peaks near $q=2 k_{F}$. The out-of-plane response $x_{z z}$ for low hole densities has the line shape associated with the response functions of an ordinary $2 \mathrm{D}$ electron system. This plateau behaviour disappears by increasing the hole density. The unusual response of the hole doped system can manifest itself in certain physical observables of monolayer $\mathrm{MoS}_{2}$. For example, we showed that for a hole doped system, the RKKY interaction between two in-plane impurity spins, calculated from an inplane response, decays with a power law $R^{-5 / 2}$ with $R$ being the distance between two impurity spins. This decay deviates from the $\mathrm{R}^{-2}$ power law seen in the twodimensional Fermi liquid. In contrast, the out-of-plane response for hole doped case, shows an $\mathrm{R}^{-2}$ behaviour. We have also calculated the Curie temperature and $\mathrm{g}$-factor for the hole doped case and showed that they can be manipulated by the magnitude of hole doping. 


\section{OVERVIEW}

In this thesis, we examined the electronic and magnetic properties of two-dimensional crystals. Our focus has been on single layer and bilayer graphene and monolayer of transition metal dichalcogenides such as $\mathrm{MoS}_{2}$. We first studied electrons and holes confined in a ring shape in bilayer graphene. Then we investigated the effect of an orbital Rashba type term induced in monolayer graphene due to an in-plane electric field. We also studied transport properties of bilayer graphene in the presence of Rashba spin-orbit coupling through calculation of chiral tunnelling in npn and np junctions. In the last chapter, we found analytical results for the q-dependent static spin susceptibility of monolayer $\mathrm{MoS}_{2}$ considering both the electron-doped and hole-doped cases. In the following, the main conclusions are summarised.

\subsection{ELECTRONIC PROPERTIES OF BILAYER GRAPHENE RINGS}

Ring conductors are interesting structures which allow us to study quantum coherent transport properties. For example, it is well known that threading a closed quantum ring with finite magnetic flux can induce a persistent current even at equilibrium. In order to study ring conductors, it is important to know the structure of the energy levels in the ring as a function of size (radius and width) and magnetic flux. Recently, monolayer graphene quantum rings have been studied, both theoretically (Recher et al., 2007) and experimentally (S. Russo and Sobhani, 2008). Lithographic cutting is one way to create a ring, which is equivalent to applying an infinite mass boundary condition in theoretical studies (Berry and Mondragon, 1987). This method has some disadvantages and limitations such as producing edge disorders, which can reduce the performance of the ring such as suppression of the persistent current (S. Russo and Sobhani, 2008; Huefner et al., 
2010). Since it is possible to induce a tuneable band gap in bilayer graphene, one can confine the charge carriers in bilayer graphene in a ring shape by applying a gate voltage or position-dependent doping. This kind of confinement is not possible in graphene due to Klein tunnelling (Katsnelson et al., 2006).

In Chap. 2, in order to study electronic properties of bilayer graphene rings, we found the energy levels of a bilayer graphene ring with finite width. We assumed that electrons and holes are confined in a ring shape by applying a bias voltage in bilayer graphene. We used a two-band Hamiltonian for describing the low energy charge carriers in bilayer graphene. Working with polar coordinates, we found the wave functions of these charge carriers in different parts of the ring. Applying boundary conditions, which in the case of two-band Hamiltonian of bilayer graphene are continuity of the wave functions and the flux probability at different interfaces, we found the energy levels in the system numerically. We showed that energy levels have a weak dependence on the ring radius when the angular momentum $|\mathrm{m}|$ is small. However as $|\mathrm{m}|$ increases, the dependence on the ring radius can be noticeable. We have also shown that for a fixed $m$, the lowest energy level as a function of magnetic field has a shape of an asymmetric Mexican hat (two minima separated by a saddle point), which is in contrast to parabolic energy levels in conventional semiconductor rings.

In order to compare our results with previous calculations (Zarenia et al., 2009), we have also used a four-band Hamiltonian and showed that for low energy limits the results are in good agreement with the results found using a two-band Hamiltonian. In our model we have neglected inter-valley scattering so that the valley index is a good quantum number. We showed that applying a magnetic flux can produce a controllable way to lift the valley degeneracy in the bilayer graphene ring. This feature can be used in valleytronics (Rycerz et al., 2007). By calculating the persistent current in a closed bilayer graphene ring in the presence of finite magnetic flux, we showed that a valley broken degeneracy can manifest itself in the persistent current. We also examined the effect of Rashba spin-orbit coupling that arises from applying gate voltage, on the energy levels in bilayer graphene ring. We showed that Rashba spin-orbit coupling breaks the 
spin degeneracy of energy levels and tends to flatten the Mexican hat shape of the lowest level. This feature can be seen in the reduction of persistent current due to decreasing the slope of the energy levels.

\subsection{ORBITAL RASHBA EFFECT IN MONOLAYER GRAPHENE}

In Chap. 3, we tried to understand the effect of an orbital Rashba-type term induced in monolayer graphene by an in-plane electric field. For this purpose we first started to see how this term can affect Landau levels in graphene. It is well known that in-plane electric fields can cause a collapse in Landau levels when $E=B v_{F}$ (Peres and Castro, 2007). However tight-binding calculations have shown that this collapse can occur even when $E<B v_{F}$ (Lukose et al., 2007). This encouraged us to see if the orbital Rashba term is responsible for this feature. By using perturbation theory, we found that up to first order, this term does not affect Landau levels. However, the second-order correction of this term to Landau levels is finite when $0<\mathrm{E}<\mathrm{B} v_{\mathrm{F}}$ and tends to repel the Landau levels. When $\mathrm{E} \rightarrow \mathrm{B} v_{\mathrm{F}}$ the correction due to orbital Rashba vanishes, so this term cannot be responsible for the early collapse found in tight-binding calculations. We also found that the $n=0$ Landau level is intact even for second-order perturbation.

We then examined how this term can affect the minimal conductivity in monolayer graphene. It has been shown (Ludwig et al., 1994) by using the Kubo formula that for clean graphene at zero temperature and finite frequency, minimal conductivity has a universal value independent of frequency given by $\sigma_{\min } / \sigma_{0}=$ $\pi / 8$ with $\sigma_{0}=g_{s} g_{v} e^{2} / 2 \pi \hbar$. By using the Kubo formula and linear response theory we showed that taking the orbital Rashba term into account, minimal conductivity can be frequency dependent. This result casts doubt upon the universality of minimal conductivity of monolayer graphene. Finally in this chapter we studied the Rabi oscillations in Landau quantised monolayer graphene in the presence of the in-plane electric field by calculating longitudinal and transverse currentcurrent correlation function. Our calculations showed that collapse and revival 
pattern in Rabi oscillations can be destroyed at sufficiently high electric fields. We also found that the orbital Rashba term has a negligible effect on the current correlation functions.

\subsection{CHIRAL TUNNELLING IN THE PRESENCE OF RASHBA SPIN-ORBIT COU- PLING}

Spin-orbit coupling is an important ingredient of spintronic studies. In graphene spin-orbit coupling has two different sources, intrinsic and extrinsic or Rashba. Intrinsic spin-orbit coupling which comes from intra-atomic spin-orbit interaction of carbon atoms in graphene is very small and in many cases negligible. However extrinsic spin-orbit coupling which can be induced for example by applying a gate voltage, can be large. Recently there have been several reports on observing huge Rashba spin-orbit coupling in graphene (Varykhalov et al., 2008). This encouraged us to study the effect of Rashba spin-orbit coupling on transport properties of bilayer graphene. To tackle this problem we have chosen chiral tunnelling as an example.

In Chap. 4, we studied the effect of Rashba spin-orbit coupling on the transport of massive chiral carriers in Bilayer graphene by considering the chiral tunnelling through electrostatic barriers. We solved the tunnelling problem by considering an incoming wave with an angle of incidence $\phi$ propagating towards the potential barrier. We then found the spinors of the reflected and transmitted waves accompanied by evanescent waves. This allowed us to find the transmission probability of the outgoing waves numerically. It is well-known that due to conservation of pseudospin, the transmission probability for normally incident electrons in bilayer graphene is zero and there are some magic angles in which the transmission probability is perfect (Katsnelson et al., 2006). We showed that in the presence of Rashba spin-orbit coupling, the bilayer graphene barrier is not transparent for any angle of incidence due to the mixture of pseudospin and spin states. We showed that for sufficiently large Rashba spin-orbit coupling, 
transmission probability for the normal incidence becomes finite and by increasing the strength of this coupling, the barrier becomes transparent for normally incident electrons. This is due to the dominance of the linear spin-orbit term in the Hamiltonian which makes the problem similar to the case of monolayer graphene.

We also have calculated the spin polarization of incident and outgoing electrons and showed that when we are in the weak Rashba spin-orbit coupling regime, the peaks of the transmission coincide with the peaks of the spin polarization. However, increasing the strength of Rashba spin-orbit coupling destroys this coincidence. We have also investigated chiral tunnelling in an npn junction with a Rashba barrier. We consider a spin-polarized incoming electron tunnelling through an npn junction in which Rashba spin-orbit coupling is only present in the barrier (Rashba barrier). We found that for weak Rashba spin-orbit coupling, the Rashba barrier can invert the spin of the incoming electron. This feature can be used in manipulating the spin of the electrons. We also showed that for incoming electrons having spin in particular direction (e.g. z), the expectation value of the spin of the outgoing electrons in z-direction has different signs for successive peaks in the transmission probability.

We have also corrected some of the results previously reported. Duppen and Peeters (2013) in their paper have claimed that transmission probability of a pn junction in bilayer and multilayer graphene can be one for some special angles. We have shown that they have made several mistakes both in choosing their wave functions for different parts of the junction and also in their physical interpretation. We showed that there are not such special angles for bilayer graphene pn junctions.

6.4 SPIN RESPONSE OF MONOLAYER TRANSITION METAL DICHALOCOGENIDES

In Chap. 5, we have calculated, and obtained analytical expressions for the wavevector-dependent static spin susceptibility of charge carriers in monolayer transi- 
tion metal dichalocogenides. Our approach is based on the effective-mass model description of electronic excitations in these materials. Very different behavior emerges for the cases of electron-doped and hole-doped systems. We illustrate our findings using band parameters for $\mathrm{MoS}_{2}$ monolayers.

Features exhibited for electron doping are similar, but not entirely analogous, to those associated with ordinary $2 \mathrm{D}$ electron gases. The finite spin-orbit coupling results in deviations from the canonical line shape near $q=2 k_{F}$. Also, unlike the ordinary $2 \mathrm{D}$ electron system, the plateau value of the spin response at small $\mathrm{q}$ depends on the electron sheet density, see Eq. (307). The total response of the electron-doped system is obtained as the sum of an extrinsic part that vanishes without the doping and an intrinsic contribution due to the completely filled valence band.

The hole-doped system shows marked deviations from the behavior expected from an ordinary $2 \mathrm{D}$ electron gas. In that, it mirrors some of the features of confined valence-band states in semiconductor heterostructures (Dietl et al., 1997; Kernreiter et al., 2013). In particular, a strong anisotropy of the spin susceptibility is exhibited, with the out-of-plane response being much stronger than the in-plane response in the low-density limit. However, the in-plane response is enhanced as the hole density increases and shows pronounced nonanalytic behavior near $\mathrm{q}=2 \mathrm{k}_{\mathrm{F}}$. We have investigated implications for spin-related physical quantities arising from the unusual spin response of the hole-doped system. We show that the oscillations of the in-plane spin response in real space decay faster than the typical $R^{-2}$ law that is expected for a $2 \mathrm{D}$ Fermi liquid. Both the Curie temperature for hole-mediated easy-axis ferromagnetism and the g-factor characterizing the Zeeman spin splitting due to an in-plane magnetic field are found to be tunable by changing the hole density.

In this work, we have neglected effects due to disorder and electron-electron interactions, which are known to, in principle, alter the spin response of ordinary 2D electron systems (De Palo et al., 2009). Parameterization in terms of local field factors (Giuliani and Vignale, 2005; De Palo et al., 2009) could be used to shed further light on how interactions renormalize the spin susceptibility of mono- 
layer transition metal dichalcogenides. As far as disorder is concerned, it can be expected that important corrections to our results obtained in the clean limit will only arise for low-enough carrier densities when the difference between $E_{F}$ and the band edge is comparable in magnitude to the disorder-induced lifetime broadening (Cappelluti et al., 2002; De Palo et al., 2005). The latter turns out to be of the order of $\sim 0.01 \mathrm{eV}$ in typical samples (Radisavljevic et al., 2011), and therefore is at least an order of magnitude smaller than all other relevant energy scales. Our work adds to the understanding of monolayer transition metal dichalcogenides as a new materials system whose charge carriers show behavior that is sometimes reminiscent of - but generally distinct from - other $2 \mathrm{D}$ systems. The very different properties exhibited by the electron-doped and holedoped cases create the possibility for a versatile engineering of electronic systems with specially tailored spin response. To verify our theoretical results, electronictransport experiments could be used to measure the carrier spin susceptibility in the $q \rightarrow 0$ limit Zhu et al. (2003); Vakili et al. (2004). Furthermore, monolayer transition metal dichalcogenides would lend themselves as ideal samples for implementing a recent proposal Stano et al. (2013) for determining the full spatial structure of the spin susceptibility.

\subsection{PUBLICATIONS RESULTING FROM THIS THESIS}

H. Hatami, T. Kernreiter, U. Zuelicke "Spin susceptibility of two-dimensional transition metal dichalcogenide" Phys. Rev. B 90, 045412 (2014).

H. Hatami, U. Zuelicke "Bilayer graphene ring in the presence of magnetic and electric fields" (in preparation for submission).

H. Hatami, U. Zuelicke "Chiral tunneling in bilayer graphene n-p-n and n-p junctions in the presence of Rashba spin orbit coupling" (in preparation).

H. Hatami, U. Zuelicke "Comments on the paper "Klein paradox for a pn junction in multilayer graphene" by B. Van Duppen and F. M. Peeters" (in preparation). 



\section{APPENDIX}

\section{A.I PROBABILITY CURRENT IN BLG IN THE PRESENCE OF RASHBA SOC}

We calculated the probability current in BLG in the presence of Rashba SOC by employing a momentum power expansion of effective Hamiltonian. This method is a smart way to define the boundary conditions especially when dealing with heterojunctions and superlattices (Li and Tao, 2007; L. W. Mlenkamp and Bauer, 2001; F. Bottegoni and Wegrowe, 2012; H. Drouhin and Wegrowe, 2011). We start with writing the Hamiltonian of the system as

$$
\mathrm{H}=\mathrm{H}_{\mathrm{RSOC}}+\mathrm{H}_{\mathrm{BLG}},
$$

where

$$
\begin{aligned}
& \mathrm{H}_{\mathrm{BLG}}=-\beta\left(\pi_{+}^{2} \sigma_{+}+\pi_{-}^{2} \sigma_{-}\right), \\
& \mathrm{H}_{\mathrm{RSOC}}=-i \bar{v}_{\mathrm{B}}\left(\pi_{+} \sigma_{+} \mathrm{S}_{-}-\pi_{-} \sigma_{-} \mathrm{S}_{+}\right) .
\end{aligned}
$$

In order to find the probability current we expand the Hamiltonian in terms of different momentum powers as following

$$
H=\sum_{i=x, y} a_{i} p_{i}+\sum_{i=x, y} \sum_{j=x, y} b_{i j} p_{i} p_{j}
$$

where $a_{i}$ and $b_{i j}$ are Hermitian operators. Hamiltonian due to Rashba spin-orbit coupling $\mathrm{H}_{\mathrm{RSOC}}$ is linear in $\mathrm{p}$ and we can write

$$
\mathrm{H}_{\mathrm{RSOC}}=\mathrm{a}_{\mathrm{x}} \mathrm{p}_{\mathrm{x}}+\mathrm{a}_{\mathrm{y}} \mathrm{p}_{\mathrm{y}}
$$

where

$$
\begin{aligned}
& a_{x}=-i \bar{v}_{B}\left(\sigma_{+} S_{-}-\pi_{-} \sigma_{-} S_{+}\right), \\
& a_{y}=-\bar{v}_{\mathrm{B}}\left(\sigma_{+} S_{-}+\pi_{-} \sigma_{-} S_{+}\right),
\end{aligned}
$$


and the parabolic term of the effective Hamiltonian can be written as

$$
H_{B L G}=b_{x x} p_{x}^{2}+b_{y y} p_{y}^{2}+b_{x y} p_{x} p_{y}+b_{y x} p_{y} p_{x}
$$

where

$$
\begin{aligned}
& b_{x x}=b_{y y}=-\beta\left(\sigma_{+}+\sigma_{-}\right), \\
& b_{x y}=b_{y x}=i \beta\left(\sigma_{+}-\sigma_{-}\right) .
\end{aligned}
$$

Now we start from continuity equation $\frac{\partial \rho}{\partial t}+\nabla . J=0$ to find the proper relation for probability current

$$
\frac{\partial \psi \psi^{*}}{\partial t}=\psi \frac{\partial \psi^{*}}{\partial t}+\frac{\partial \psi}{\partial t} \psi^{*}
$$

So from the Schrödinger equation $i \hbar \frac{\partial}{\partial t} \psi=H \psi$ we have

$$
\begin{aligned}
\frac{\partial \psi \psi^{*}}{\partial t}= & \frac{1}{i \hbar}\left(\psi^{*} H \psi-\psi(H \psi)^{*}\right)= \\
& \frac{1}{i \hbar}\left\{\psi^{*} H_{R S O C} \psi-\left(H_{R S O C} \psi\right)^{*} \psi+\psi^{*} H_{B L G} \psi-\left(H_{B L G} \psi\right)^{*} \psi\right\} .
\end{aligned}
$$

First we find the probability current due to $\mathrm{H}_{\mathrm{RSOC}}$

$$
\begin{aligned}
\frac{1}{i \hbar}\left\{\psi^{*} H_{R S O C} \psi-\left(H_{R S O C} \psi\right)^{*} \psi\right\}= & \frac{1}{i \hbar}\left\{\psi^{*} \sum_{i=x, y} a_{i} p_{i} \psi-\left(\sum_{i=x, y} a_{i} p_{i} \psi\right)^{*} \psi\right\} \\
& =\frac{1}{i \hbar}\left\{\psi^{*} \sum_{i=x, y} a_{i} p_{i} \psi+\sum_{i=x, y} p_{i} \psi^{*} a_{i} \psi\right\}
\end{aligned}
$$

So from the continuity equation the current probability can be written as

$$
\nabla . J=\nabla_{x} J_{x}+\nabla_{y} J_{y}=-\frac{1}{i \hbar} \sum_{i=x, y}\left(\psi^{*} a_{i} p_{i} \psi+p_{i} \psi^{*} a_{i} \psi\right) \text {. }
$$

By using the differential form of the momentum operator $p_{i}=\frac{\hbar}{i} \nabla_{i}$ we have

$$
\nabla . J=\sum_{i=x, y}\left(\psi^{*} a_{i} \nabla_{i} \psi+\nabla_{i} \psi^{*} a_{i} \psi\right)=\sum_{i=x, y} \nabla_{i}\left(\psi^{*} a_{i} \psi\right)
$$


The current probability due to linear term of the effective Hamiltonian $\mathrm{H}_{\mathrm{RSOC}}$ is therefore given by

$$
\mathrm{J}_{i}^{\mathrm{L}}=\psi^{*} \mathrm{a}_{i} \psi
$$

and by using the similar procedure we can find the probability current for the parabolic term $\mathrm{H}_{\mathrm{BLG}}$

$$
\mathrm{J}_{i}^{P}=\psi^{*} b_{i i} p_{i} \psi+\psi^{*} b_{i j} p_{j} \psi
$$

Thus the probability current in $i$ direction for BLG in the presence of Rashba SOC is given by

$$
J_{i}=J_{i}^{L}+J_{i}^{P}=\psi^{*} a_{i} \psi+\psi^{*} b_{i i} p_{i} \psi+\psi^{*} b_{i j} p_{j} \psi
$$





\section{BIBLIOGR A PHY}

M. Abramowitz and I. A. Stegun. Handbook of Mathematical Functions with Formulas, Graphs, and Mathematical Tables. Dover Publications, National Bureau of Standards, Washington, 1964.

S. Adam, E. H. Hwang, V. Galitski, and S. D. Sarma. Proc. Natl. Acad. Sci., 104: $18392,2007$.

Y. Aharonov and D. Bohm. Phys. Rev., 115:485, 1959.

P. Allian and J. Fuchs. Eur.Phys. J. B, 83:301, 2011.

a. T. N. Ando, T. and R. Saito. J. Phys. Soc. Jpn., 67:2857, 1998.

T. Ando. J. Phys. Soc. Jpn., 75:074716, 2006.

T. Ando. NPG Asia Mater., 1:17, 2009.

T. Ando, A. B. Fowler, and F. Stern. Rev. Mod. Phys., 54:437, 1982.

N. W. Ashcroft and V. D. Mermin. Solid State Physics. 1976.

D. Bercioux and A. D. Martino. Phys. Rev. B, 81:165410, 2010.

J. Bernád, U. Zülicke, and K. Ziegler. Physica E, 42:755, 2010.

M. V. Berry and R. J. Mondragon. Proc. R. Soc. Lond. A, 412:53, 1987.

K. I. Bolotin, F. Ghahari, M. D. Shulman, H. L. Stormer, and P. Kim. Nature, 462: 196, 2009.

L. Brey, H. A. Fertig, and S. Das Sarma. Phys. Rev. Lett., 99:116802, 2007.

M. Büttiker, Y. Imry, and R. Landauer. Phys. Lett. A, 96:365, 1983. 
X. W. C. Lee, J. W. Kysar, and J. Hone. Science, 321:385, 2008.

A. Calogeracos and N. Dombey. Contemp. Phys., 40:313, 1999.

T. Cao, G. Wang, W. Han, H. Ye, C. Zhu, J. Shi, Q. Niu, P. Tan, E. Wang, B. Liu, and J. Feng. Nat. Comms., 3:887, 2012.

E. Cappelluti, C. Grimaldi, and L. Pietronero. Eur. Phys. J. B, 30:511, 2002.

V. Cheianov and V. I. Falḱko. Phys. Rev. B, 74:041403, 2006.

V. V. Cheianov, V. Falḱko, and B. L. Altshuler. Science, 315:1252, $2007 \mathrm{a}$.

V. V. Cheianov, V. I. Falko, B. L. Altshuler, and I. L. Aleiner. Phys. Rev. Lett., 99: 176801, 2007b.

Y. C. Cheng, Z. Y. Zhu, W. B. Mi, Z. B. Guo, and U. Schwingenschlögl. Phys. Rev. $B, 87: 100401,2013$.

D. R. Cooper, B. DÁnjou, N. Ghattamaneni, B. Harack, M. Hilke, A. Horth, N. Majlis, M. Massicotte, L. Vandsburger, E. Whiteway, and V. Yu. ISRN Cond. Matt. Phys., 2012:501686, 2012.

J. Cserti. Phys. Rev. B, 75:033405, 2007.

A. L. C. P. D. A. Bahamon and P. A. Schulz. Phys. Rev. B, 79:125414, 2009.

A. Dato, Z. Lee, K. Jeon, R. Erni, V. Radmilovic, T. J. Richardsonc, and M. Frenklachd. Chem. Commun., page 6095, 2009.

S. De Palo, M. Botti, S. Moroni, and G. Senatore. Phys. Rev. Lett., 94:226405, 2005.

S. De Palo, S. Moroni, and G. Senatore. J. Phys. A: Math. Theor., 42:214013, 2009.

Y. S. Dedkov, M. Fonin, U. Rudiger, and C. Laubschat. Phys. Rev. Lett., 100:107602, 2008.

T. Dietl, A. Haury, and Y. M. d'Aubigné. Phys. Rev. B, 55:R3347, 1997. 
K. Dolui, I. Rungger, C. Das Pemmaraju, and S. Sanvito. Phys. Rev. B, 88:075420, 2013.

B. Dóra, K. Ziegler, P. Thalmeier, and M. Nakamura. Phys. Rev. Lett., 102:036803, 2009 .

X. Du, I. Skachko, F. Duerr, A. Luican, and E. Y. Andrei. Nature, 462:192, 2009.

B. V. Duppen and F. M. Peeters. Europhys. Lett., 102:27001, 2013.

a. G. F. F. Bottegoni, and H. Drouhin and J. Wegrowe. Phys. Rev. B, 85:235313, 2012.

A. Geim. Science, 324:1530, 2009.

A. Geim and K. Novoselov. Nat. Mater., 6:183, 2007.

R. Gelderen and C. M. Smith. Phys. Rev. B, 81:125435, 2010.

G. Giuliani and G. Vignale. Quantum Theory of the Electron Liquid. Cambridge University Press, Cambridge, UK, 2005.

M. Gmitra, S. Konschuh, C. Ertler, C. Ambrosch-Draxl, and J. Fabian. Phys. Rev. $B, 80: 235431,2009$.

F. Guinea. New J. Phys., 12:083063, 2010.

a. G. F. H. Drouhin and J. Wegrowe. Phys. Rev. B, 83:113307, 2011.

M. Huefner, F. Molitor, A. Jacobsen, A. Pioda, C. Stampfer, K. Ensslin, and T. Ihn. New J. Phys., 12:043054, 2010.

E. H. Hwang and S. Das Sarma. Phys. Rev. B, 75:205418, 2007.

E. H. Hwang and S. Das Sarma. Phys. Rev. Lett., 101:156802, 2008.

S. Iijima et al. Nature, 354:56, 1991.

S. W. K. Ismail and K. Y. Lee. Appl. Phys. Lett., 59:1998, 1991. 
C. L. Kane and E. J. Mele. Phys. Rev. Lett., 95:226801, 2005.

T. Kasuya. Prog. Theor. Phys., 16:45, 1956.

M. I. Katsnelson, K. S. Novoselov, and A. K. Geim. Nat. Phys., 2:620, 2006.

T. Kernreiter, M. Governale, and U. Zülicke. Phys. Rev. Lett., 110:026803, 2013.

K. S. Kim, Y. Zhao, H. Jang, S. Y. Lee, J. M. Kim, K. S. Kim, J.-H. Ahn, P. Kim, J. Y. Choi, and B. H. Hong. Nature, 457:706, 2009.

D. F. Kirwan, C. G. Rocha, A. T. Costa, and M. S. Ferreira. Phys. Rev. B, 77:085432, 2008.

S. Konschuh, M. Gmitra, and J. Fabian. Phys. Rev. B, 82:245412, 2010.

S. Konschuh, D. Kochan, and J. Fabian. Phys. Rev. B, 85:115423, 2012.

A. Kormányos, V. Zólyomi, N. D. Drummond, P. Rakyta, G. Burkard, and V. I. Fal'ko. Phys. Rev. B, 88:045416, 2013.

H. W. Kroto et al. Nature, 3:162, 1985.

a. G. S. L. W. Mlenkamp and G. E. W. Bauer. Phys. Rev. B, 64:121202(R), 2001.

L. D. Landau. Phys. Z. Sowjet., 11:26, 1937.

R. Laughlin. Phys. Rev. B, 23:5632, 1981.

Y. Li and R. Tao. Phys. Rev. B, 75:075319, 2007.

T. Low, H. Seokmin, and a. M. L. J. Appenzeller, and S. Datta. IEEE Trans. Electron Devices, 56:1292, 2009.

H.-Z. Lu, W. Yao, D. Xiao, and S.-Q. Shen. Phys. Rev. Lett., 110:016806, 2013.

A. W. W. Ludwig, M. P. A. Fisher, R. Shankar, and G. Grinstein. Phys. Rev. B, 50: 7526, 1994 .

V. Lukose, R. Shankar, and G. Baskaran. Phys. Rev. Lett., 98:116802, 2007. 
a. J. B. M. Liu and K. Richter. Phys. Rev. B, 85:085406, 2012.

O. Madelung. Introduction to Solid-State Theory. Springer, Berlin, 1978.

K. F. Mak, C. Lee, J. Hone, J. Shan, and T. F. Heinz. Phys. Rev. Lett., 105:136805, 2010.

E. McCann. Phys. Rev. B, 74:161403, 2006.

E. McCann and V. I. Falko. Phys. Rev. Lett., 96:086805, 2006.

E. McCann and M. Koshino. Rep. Prog. Phys., 76:056503, 2013.

N. Mermin. Phys. Rev., 176:250, 1968.

F. Miao, S. Wijeratne, Y. Zhang, U. C. Coskun, W. Bao, and C. N. Lau. Science, 317:1530, 2007 .

H. Min, J. E. Hill, N. A. Sinitsyn, B. R. Sahu, L. Kleinman, and A. H. MacDonald. Phys. Rev. B, 74:165310, 2006.

F. Mireles and J. Schliemann. New J. Phys., 14:093026, 2012.

R. Mishra, W. Zhou, S. J. Pennycook, S. T. Pantelides, and J.-C. Idrobo. Phys. Rev. $B, 88: 144409,2013$.

G. E. Moore. Solid-State Circuits Society Newsletter, IEEE, 11:33, 2006.

T. Moriya. Spin Fluctuations in Itinerant Electron Magnetism. Springer, Berlin, 1985.

S. V. Morozov, K. S. Novoselov, M. Katsnelson, F. Schedin, D. Elias, and A. G. J.A. Jaszczak. Phys. Rev. Lett., 100:016602, 2008.

A. H. C. Neto, F. Guinea, N. M. R. Peres, K. S. Novoselov, and A. K. Geim. Rev. Mod. Phys., 81:109, 2009.

J. Nilsson, A. H. C. Neto, F. Guinea, and N. M. R. Peres. Phys. Rev. B, 76:165416, 2007.

J. Nitta. NTT Tech. Rev., 2:31, 2004. 
J. Nitta, F. E. Meijer, and H. Takayanagi. Appl. Phys. Lett., 75:695, 1999.

K. S. Novoselov, A. K. Geim, S. Morozov, D. Jiang, Y. Zhang, S. Dubonos, I. Grigorieva, and A. Firsov. Science, 306:666, 2004.

K. S. Novoselov, A. K. Geim, S. Morozov, D. Jiang, I. G. M. I. Katsnelson, S. Dubonos, and A. Firsov. Nature, 438:197, 2005a.

K. S. Novoselov, D. Jiang, F. Schedin, V. K. T. J. Booth, S. Morozov, and A. K. Geim. natl. Acad. Sci., 102:10451, 2005b.

K. S. Novoselov, Z. Jiang, Y. Zhang, S. V. Morozov, H. L. Stormer, U. Zeitler, J. C. Maan, G. S. Boebinger, P. Kim, and A. K. Geim. Science, 315:1379, 2007.

H. Ochoa and R. Roldán. Phys. Rev. B, 87:245421, 2013.

H. Ochoa, F. Guinea, and V. I. Falko. Phys. Rev. B, 88:195417, 2013.

T. Ohta, A. Bostwick, T. Seyller, K. Horn, and E. Rotenberg. Science, 313:951, 2006.

F. Parhizgar, H. Rostami, and R. Asgari. Phys. Rev. B, 87:125401, 2013.

R. E. Peierls. Helv. Phys. Acta, 7:81, 1923.

N. M. R. Peres and E. V. Castro. J. Phys.: Condens. Matter, 19:406231, 2007.

R. Prange and S. M. Girvin. The Quantum Hall Effect. 1987.

P. K. Pyatkovskiy. J. Phys.: Condens. Matter, 21:025506, 2009.

Z. Qiao, X. Li, W. Tse, H. Jiang, Y. Yao, and Q. Niu. Phys. Rev. B, 87:125405, 2013.

B. Radisavljevic, A. Radenovic, J. Brivio, V. Giacometti, and A. Kis. Nat. Nanotech., 6:147, 2011.

E. I. Rashba. Phys. Rev. B, 79:161409R, 2009.

P. Recher, B. Trauzettel, A. Rycerz, Y. M. Blanter, C. W. J. Beenakker, and A. F. Morpurgo. Phys. Rev. B, 76:235404, 2007. 
S. Reich, J. Maultzsch, C. Thomsen, and P. Ordejon. Phys. Rev. B, 66:035412, 2002.

H. Rostami, A. G. Moghaddam, and R. Asgari. Phys. Rev. B, 88:085440, 2013.

M. A. Ruderman and C. Kittel. Phys. Rev., 96:99, 1954.

A. Rycerz and C. W. J. Beenakker. Arxiv, page 0709.3397V1, 2007.

A. Rycerz, J. T. Strokeo, and C. W. J. Beenakker. Nat. Phys., 3:172, 2007.

D. W. H. B. H. S. Russo, J. B. Oostinga and S. S. Sobhani. Phys. Rev. B, 77:085413, 2008.

R. Saito, G. Dresselhaus, and M. S. Dresselhaus. Physical properties of carbon nanotubes. 1998.

J. J. Sakurai. Modern Quantum Mechanics. Addison-Wesley, 1994.

S. Saremi. Phys. Rev. B, 76:184430, 2007.

A. Scholz and J. Schliemann. Phys. Rev. B, 83:235409, 2011.

A. Scholz, T. Stauber, and J. Schliemann. Phys. Rev. B, 86:195424, 2012.

A. Scholz, T. Stauber, and J. Schliemann. Phys. Rev. B, 88:035135, 2013.

F. Schwierz. Nat. Nanotechnol., 5:487, 2010.

J.-H. She and A. R. Bishop. Phys. Rev. Lett., 111:017001, 2013.

P. Simon, B. Braunecker, and D. Loss. Phys. Rev. B, 77:045108, 2008.

J. C. Slonczewski and P. R. Weiss. Phys. Rev., 109:272, 1958.

Y. Song and H. Dery. Phys. Rev. Lett., 111:026601, 2013.

P. Stano, J. Klinovaja, A. Yacoby, and D. Loss. Phys. Rev. B, 88:045441, 2013.

A. D. Stone and Y. Imry. Phys. Rev. Lett., 56:189, 1986.

K. Suzuki and J. C. Hensel. Phys. Rev. B, 9:4184-4218, 1974. 
a. A. P. I. T. Luo, H. A. Fertig, and L. Brey. Phys. Rev. B, 80:165310, 2009.

Y.-W. Tan, Y. Zhang, K. Bolotin, Y. Zhao, S. Adam, E. H. Hwang, S. D. Sarma, H. L. Stormer, and P. Kim. Phys. Rev. Lett., 99:246803, 2007.

G. Timp, A. M. Chang, and J. E. Cunningham. Phys. Rev. Lett., 58:2824, 1987.

T. Tudorovskiy and M. Katsnelson. Phys. Scr., T146:014010, 2012.

J. Tworzydlo, B. Trauzettel, M. Titov, A. Rycerz, and C. Beenakker. Phys. Rev. Lett., 96:246802, 2006.

B. Uchoa, T. G. Rappoport, and A. H. Castro Neto. Phys. Rev. Lett., 106:016801, 2011.

K. Vakili, Y. P. Shkolnikov, E. Tutuc, E. P. De Poortere, and M. Shayegan. Phys. Rev. Lett., 92:226401, 2004.

A. Varykhalov, J. Sanchez-Barriga, A. M. Shikin, C. Biswas, E. Vescovo, A. Rybkin, D. Marchenko, and O. Rader. Phys. Rev. Lett., 101:157601, 2008.

P. R. Wallace. Phys. Rev., 71:622, 1947.

L. Wang and M. W. Wu. arXiv:1312.6985.

Q. H. Wang, K. Kalantar-Zadeh, A. Kis, J. N. Coleman, and M. S. Strano. Nat. Nanotech., 7:699, 2012.

X. Wang, Q. Li, J. Xie, Z. Jin, J. Wang, Y. Li, K. Jiang, and S. Fan. Nano Let., 9:3137, 2009 .

S. Washburn and R. Webb. Adv. Phys., 35:375, 1986.

R. A. Webb, S. Washburn, and C. P. Umbach. Phys. Rev. Lett., 54:2696, 1985.

C. Weeks, J. A. J. Hu, M. Franz, and R. Q. Wu. Phys. Rev. X, 1:021001, 2011.

R. Winkler. Spin-Orbit Coupling Effects in Two-Dimensional Electron and Hole Systems. Springer, Berlin, 2003. 
R. Winkler and U. Zülicke. Phys. Rev. B, 82:245313, 2010.

R. Winkler and U. Zülicke. Arxiv, page 1206.4761v1, 2012.

B. Wunsch, T. Stauber, F. Sols, and F. Guinea. New J. Phys., 8:318, 2006.

J. Wurm, M. Wimmer, H. U. Baranger, and K. Richter. Semicond. Sci. Technol., 825: 034003, 2010.

D. Xiao, W. Yao, and Q. Niu. Phys. Rev. Lett., 99:236809, 2007.

D. Xiao, G.-B. Liu, W. Feng, X. Xu, and W. Yao. Phys. Rev. Lett., 108:196802, 2012.

A. Yamakage, K. Imura, J. Cayssol, and Y. Kuramoto. Europhys. Lett., 87:47005, 2009.

K. Yosida. Phys. Rev., 106:893, 1957.

K. Yosida. Theory of Magnetism. Springer, Berlin, 1996.

A. F. Young and P. Kim. Nature, 5:222, 2009.

T. Yu and M. W. Wu. arXiv:1401.0047.

M. Zarea and N. Sandler. Phys. Rev. B, 79:165442, 2009.

M. Zarenia, J. M. Pereira, F. M. P. Jr., and G. A. Farias. Nanolett., 9:4088, 2009.

M. Zarenia, J. M. Pereira, A. C. Jr., F. M. Peeters, and G. A. Farias. Phys. Rev. B, 81:045431, 2010 .

M. Zarenia, J. M. Pereira, A. C. Jr., F. M. Peeters, and G. A. Farias. Phys. Rev. B, 82:119906(E), 2010b.

H. Zeng, J. Dai, W. Yao, D. Xiao, and X. Cui. Nat. Nanotech., 7:490, 2012.

Y. Zhang, Y.-W. Tan, H. L. Stormer, and P. Kim. Phys. Rev. B, 438:201, 2005.

Y. Zheng and T. Ando. Phys. Rev. B, 65:245420, 2002. 
J. Zhu, H. L. Stormer, L. N. Pfeiffer, K. W. Baldwin, and K. W. West. Phys. Rev. Lett., 90:056805, 2003.

K. Ziegler. Phys. Rev. B, 55:10661, 1997.

K. Ziegler. Phys. Rev. Lett., 80:3113, 1998.

K. Ziegler. Phys. Rev. Lett., 97:266802, 2006.

K. Ziegler. Phys. Rev. B, 75:233407, 2007.

I. Zutic, J. Fabian, and S. D. Sarma. Rev. Mod. Phys., 76:323, 2004. 